Redalyc 3.0

ESCI Web of science

UNIVERSIDAD POLITÉCNICA SALESIANA ECUADOR

enero / junio 2021
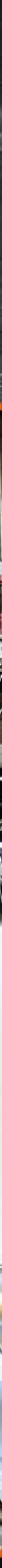

Análisis del HF0-1234ze como alternativa ecológica en la refrigeración doméstica

Pag. 9
Evaluación de un radar FMCW como herramienta didáctica en las carreras de Ingeniería Automotriz y Telecomunicaciones Pag. 70
Diseño y construcción de un reactor discontinuo con recirculación externa para obtener biodiésel a partir de aceite de fritura en condiciones subcríticas Pag. 32
Análisis de vulnerabilidades con SQLMAP aplicada a entornos APEX 5

Pag. 104 


\section{INGENIUS}

Ingenius • Número $25 \bullet$ enero/junio 2021. Revista semestral de Ciencia y Tecnología de la Universidad Politécnica Salesiana del Ecuador. Publicación dedicada a estudios relacionados con las Ciencias de la Ingeniería Mecánica, Ingeniería Eléctrica, Ingeniería Eléctronica, Ingeniería Mecatrónica, Ciencias de la computación y áreas afines.

\section{Consejo de Editores}

Rafael Antonio Balart Gimeno, PhD, Universidad Politécnica de Valencia, España - Editor Jefe.

\section{Consejo Científico}

Juan López Martínez, PhD, Universidad Politécnica de Valencia, España.

Elena Fortunati, PhD, Universidad de Perugia, Italia.

Gustavo Rovelo Ruiz, PhD, Hasselt University, Diepenbeek, Bélgica.

Franklin Gavilanez Alvarez, PhD, American University, Estados Unidos.

Piedad Gañan Rojo, PhD, Universidad Pontificia Bolivariana, Colombia.

José Alex Restrepo, PhD, Universidad Simón Bolívar, Venezuela.

Sergio Lujan Mora, PhD, Universidad de Alicante, España.

Martha Zequera Díaz, PhD, Pontificia Universidad Javeriana, Colombia.

Grover Zurita, PhD, Universidad Privada Boliviana, Bolivia.

Vladimir Robles, PhD, Universidad Politécnica Salesiana, Ecuador.

Germán Arévalo, PhD, Universidad Politécnica Salesiana, Ecuador.

Wilbert Aguilar, PhD, Universidad de las Fuerzas Armadas, ESPE, Ecuador.

Pablo Parra Rosero, PhD, Universidad Politécnica Salesiana, Ecuador.

JaCk Bravo Torres, PhD, Universidad Politécnica Salesiana, Ecuador.

Walter Orozco, PhD, Universidad Politécnica Salesiana, Ecuador.

Mariela Cerrada, PhD, Universidad Politécnica Salesiana, Ecuador.

Julio CÉsar Viola, PhD, Universidad Politécnica Salesiana, Ecuador.

Sergio Gamboa SÁnchez, PhD, Universidad Nacional Autónoma de México, México.

Roger Abdón Bustamante Plaza, PhD, Universidad de Chile, Chile.

Christian Blum, PhD, Consejo Superior de Investigaciones Científicas, España.

Silvia Noemi Schiaffino, PhD, Universidad Nacional del Centro de la Provincia de Buenos Aires, Argentina.

Analía Adriana Amandi, PhD, Universidad Nacional del Centro de la Provincia de Buenos Aires, Argentina.
John Ignacio Calle Sigüencia, PhD, Universidad Politécnica Salesiana, Ecuador - Editor Jefe.

Marlon Xavier Quinde Abril, MSc, Universidad Politécnica Salesiana, Ecuador - Editor Asociado.
Rubén de Jesús Medina Molina, PhD, Universidad de Los Andes, Venezuela.

Johnny Josué Bullón Torrealba, PhD, Universidad de Los Andes, Venezuela.

Rodrigo Palma Hillerns, PhD, Universidad de Chile, Chile.

Gerardo Espinoza Pérez, PhD, Universidad Nacional Autónoma de México, México. Alexandre Mendes Abrão, PhD, Universidad Federal de Minas Gerais, Brasil.

Kamla Abdel Radi Ismail, PhD, Universidad Estatal de Campinas Unicamp, Brasil.

Arnaldo Da Silva, PhD, Universidad Estatal de Campinas Unicamp, Brasil.

Álvaro Rocha, PhD, Universidad de Coimbra, Portugal.

José Antenor Pomilio, PhD, Universidad Estatal de Campinas Unicamp, Brasil.

Luis Paulo Reis, PhD, Universidad de Minho, Portugal.

Luís Fernandes, PhD, Escuela Superior Náutica Infante d. Henrique, Portugal.

Aníbal Traça de Almeida, PhD, Universidad de Coimbra, Portugal.

Jorge SÁ Silva, PhD, Universidad de Coimbra, Portugal.

Pedro Manuel Soares Moura, PhD, Universidad de Coimbra, Portugal.

Sérgio Manuel Rodrigues Lopes, PhD, Universidad de Coimbra, Portugal.

Ricardo Madeira Soares Branco, PhD, Universidad de Coimbra, Portugal.

Carlos Alexandre Bento Capela, PhD, Universidad de Coimbra, Portugal.

Filipe Araujo, PhD, Universidad de Coimbra, Portugal.

Luis Manuel Guerra Silva Rosa, PhD, Universidad de Lisboa, Portugal.

Hélder de Jesus Fernandes, Puga, PhD, Universidad de Minho, Portugal.

Filipe Samuel, Pereira da Silva, PhD, Universidad de Minho, Portugal.

César Sequeira, PhD, Universidad de Lisboa, Portugal.

José Teixeira Estêvão Ferreira, PhD,
Universidad de Coimbra, Portugal.

Nuno Laranjeiro, PhD, Universidad de Coimbra, Portugal.

Luís Amaral, PhD, Universidad de Lisboa, Portugal.

Jorge Henriques, PhD, Universidad de Coimbra, Portugal.

William Ipanaque, PhD, Universidad de Piura, Perú.

Lorenzo Leija Salas, PhD, Centro de Investigación y Estudios Avanzados del Instituto Politécnico Nacional, México.

Valeri Kontorovich Mazover, PhD, Centro de Investigación y de Estudios Avanzados del Instituto Politécnico Nacional, México.

Alejandro Ávila García, PhD, Centro de Investigación y de Estudios Avanzados del Instituto Politécnico Nacional, México.

Paolo Bellavista, PhD, Universidad de Bologna, Italia.

Carlos Rubio, PhD, Centro de Ingeniería y Desarrollo Industrial, México.

Fernando Hernández Sánchez, PhD, Centro de Investigación Científica de $\mathrm{Yu}$ catán, México.

Emilio Muñoz Sandoval, PhD, Instituto Potosino de Investigación Científica y Tecnológica, México.

Yasuhiro Matsumoto Kuwabara, PhD, Centro de Investigación y de Estudios Avanzados del Instituto Politécnico Nacional, México.

David Zumoffen, PhD, Centro Internacional Franco Argentino de Ciencias de la Información y de Sistemas, Argentina.

Vicente Rodríguez GonzÁlez, PhD, Instituto Potosino de Investigación Científica y Tecnológica, México.

Alejandro Rodríguez Ángeles, PhD, Centro de Investigación y de Estudios Avanzados del Instituto Politécnico Nacional, México.

Alistair Borthwick, PhD, Universidad de Edimburgo, Reino Unido.

Copyright. Ingenius 2021, Universidad Politécnica Salesiana. Se permite la reproducción total o parcial de esta revista citando la fuente. 
Federico Dominguez, PhD, Escuela Superior Politécnica del Litoral, Ecuador.

Enrique Carrera, PhD, Universidad de las Fuerzas Armadas, ESPE, Ecuador.

Andrés Tello, MSc, Universidad de Cuenca, Ecuador.

Cristian García Bauza, PhD, Universidad Nacional del Centro de la Provincia de Buenos Aires, Argentina.

Osvaldo AÑó, PhD, Universidad Nacional de San Juan, Argentina.

Thalía San Antonio, PhD, Universidad Técnica de Ambato, Ecuador.

Victor Saquicela, PhD, Universidad de Cuenca, Ecuador.

Gonzalo Olmedo, PhD, Universidad de las Fuerzas Armadas, ESPE, Ecuador.

Román Lara, PhD, Universidad de las Fuerzas Armadas, ESPE, Ecuador.

Guillermo Soriano, PhD, Escuela Superior Politécnica del Litoral, Ecuador.

María Fernanda Granda, PhD, Universidad de Cuenca, Ecuador.

Ricardo Cayssials, PhD, Universidad Tecnológica Nacional, Argentina.

Leonardo Solaque Guzman, PhD, Universidad Militar Nueva Granada, Colombia.

José Di PAOLO, PhD, Universidad Nacional de Entre Ríos, Argentina.

Astrid Rubiano Fonseca, PhD, Universidad Militar Nueva Granada, Colombia.

Robinson Jiménez, PhD, Universidad Militar Nueva Granada, Colombia.

Alfonso Zozaya, PhD, Universidad de Carabobo, Venezuela.

Mauricio Mauledoux, PhD, Universidad Militar Nueva Granada, Colombia.

Luis Medina, PhD, Universidad Simón Bolivar, Venezuela.

Ernesto Cuadros-Vargas, PhD, Universidad Católica San Pablo, Perú.

Samuel Sepúlveda Cuevas, PhD, Universidad de la Frontera, Chile.

Carlos Cares, PhD, Universidad de la Frontera, Chile.

RAfael Sotelo, PhD, Universidad de Montevideo, Uruguay.

Omar Lopez, PhD, Universidad de Los Andes, Colombia.

Job Flores-Godoy, PhD, Universidad Católica del Uruguay, Uruguay.

Luis Mario Mateus, PhD, Universidad de los Andes, Colombia.

Amadeo Argüelles Cruz, PhD, Instituto Politécnico Nacional, México.

Santiago Bentancourt Parra, PhD, Universidad Pontificia Bolivariana, Colombia.

Germán Zapata, PhD, Universidad Nacio- nal de Colombia, Colombia.

Pedro García, PhD, Universidad Autónoma de Barcelona, España.

Arturo Conde Enríquez, PhD, Universidad Autónoma de Nuevo León, México.

Alberto Cavazos González, PhD, Universidad Autónoma de Nuevo León, México.

Ernesto VÁzquez Martínez, PhD, Universidad Autónoma de Nuevo León, México.

Miguel Díaz Rodriguez, PhD, Universidad de Los Andes, Venezuela.

Efraín Alcorta García, PhD, Universidad Autónoma de Nuevo León, México.

Luis Chirinos Garcia, PhD, Pontificia Universidad Católica de Perú, Perú.

Oscar Avilés, PhD, Universidad Militar Nueva Granada, Colombia.

Dora Martínez Delgado, PhD, Universidad Autónoma de Nuevo León, México.

David Ojeda, PhD, Universidad Técnica del Norte, Ecuador.

Irene Beatríz Steinmann, PhD, Universidad Tecnológica Nacional, Argentina.

Mario Serrano, Universidad Nacional de San Juan, Argentina.

Cornelio Posadas Castillo, PhD, Universidad Autónoma Nuevo León, México.

Mario Alberto Rios Mesias, PhD, Universidad de Los Andes, Colombia.

Yudith Cardinale Villarreal, PhD, Universidad Simón Bolivar, Venezuela.

Eduardo Matallanas, PhD, Universidad Politécnica de Madrid, España.

Jose Eduardo Ochoa Luna, PhD, Universidad Católica San Pablo, Perú.

Dante Angel Elias Giordano, PhD, Pontificia Universidad Católica de Perú, Perú.

Manuel Pelaez Samaniego, PhD, Universidad de Cuenca, Ecuador.

Juan Espinoza Abad, PhD, Universidad de Cuenca, Ecuador.

Pietro Codara, PhD, Universidad de Milan, Italia.

Alberto Soria, PhD, Centro de Investigación y de Estudios Avanzados del Instituto Politécnico Nacional, México.

José M. Aller, PhD, Universidad Politécnica Salesiana, Ecuador.

Ferney Amaya F., PhD, Universidad Pontificia Bolivariana, Medellin, Colombia.

Santiago Arango Aramburo, PhD, Universidad Nacional de Colombia, Colombia.

Diego Arcos-Avilés, PhD, Universidad de las Fuerzas Armadas, ESPE, Ecuador.

Pablo Arevalo, PhD, Universidad Politécnica Salesiana, Ecuador.

Roberto Beltran, MSc, Universidad de las Fuerzas Armadas, ESPE, Ecuador.
Leonardo Betancur, PhD, Universidad Pontificia Bolivariana, Medellin, Colombia.

Roberto Gamboa, PhD, Universidad de Lisboa, Portugal.

Paulo Lopes dos Santos, PhD, Universidad do Porto, Portugal.

Pedro André Dias Prates, PhD, Universidad de Coimbra, Portugal.

José Manuel Torres Farinha, PhD, Universidad de Coimbra, Portugal.

Celso de Almeida, PhD, Universidad Estatal de Campinas Unicamp, Brasil.

Ramon Molina Valle, PhD, Universidad Federal de Minas Gerais, Brasil.

Cristina Nader Vasconcelos, PhD, Universidad Federal Fluminense, Brasil.

João M. Ferreira Calado, PhD, Universidad de Lisboa, Portugal.

Guilherme Luz Tortorella, PhD, Universidad Federal de Santa Catarina, Brasil.

Mauro E. Benedet, PhD, Universidad Federal de Santa Catarina, Brasil.

Artemis Marti Ceschin, PhD, Universidade de Brasilia, Brasil.

Gilmar Barreto, PhD, Universidad Estatal de Campinas Unicamp, Brasil.

Ricardo Emilio F. Quevedo Nogueira, PhD, Universidad Federal de Ceará, Brasil.

Weslley Luiz da Silva Assis, PhD, Universidad Federal Fluminense, Brasil.

Ana P. Martinazzo, PhD, Universidad Federal Fluminense, Brasil.

Jorge Bernardino, PhD, Universidad de Coimbra, Portugal.

Luis Geraldo Pedroso Meloni, PhD, Universidad Estatal de Campinas Unicamp, Brasil.

Facundo Almeraya Calderón, PhD, Universidad Autónoma de Nuevo León, México.

Freddy Villao Quezada, PhD, Escuela Superior Politécnica del Litoral, Ecuador.

Jose Manrique Silupu, MSc, Universidad de Piura, Perú.

Germán Ariel Salazar, PhD, Instituto de Investigaciones en Energía no Convencional, Argentina.

José Mahomar Jananías, PhD, Universidad del BIOBIO, Chile.

Arnaldo Jélvez Caamaño, PhD, Universidad del BIOBIO, Chile.

Jorge Andrés Uribe, MSc, Centro de Ingeniería y Desarrollo Industrial, México.

Ricardo Beltran, PhD, Centro de Investigación en Materiales Avanzados, México.

Adi Corrales, MSc, Centro de Ingeniería y Desarrollo Industrial, México.

Jorge Uribe Calderón, PhD, Centro de Investigación Científica de Yucatán, México 
José Trinidad Holguín Momaca, MSc, Centro de Investigación en Materiales Avanzados, México.

Juan Manuel Alvarado Orozco, PhD, Centro de Ingeniería y Desarrollo Industrial, México.

Arnaldo Jélvez Caamaño, PhD, Universidad del BIOBIO, Chile.

Javier Murillo, PhD, Centro Internacional Franco Argentino de Ciencias de la Información y de Sistemas, Argentina.

Lucas Daniel Terissi, PhD, Universidad Nacional de Rosario, Argentina.

Rene Vinicio Sanchez Loja, PhD, Universidad Politécnica Salesiana, Ecuador.

Freddy Leonardo Bueno Palomeque, MSc, Universidad Politécnica Salesiana, Ecuador.

Diego Cabrera Mendieta, PhD, Universidad Politécnica Salesiana, Ecuador.

Edwuin Jesus Carrasquero, PhD, Universidad Técnica de Machala, Ecuador.

Carlos Mauricio Carrillo Rosero, MSc, Universidad Técnica de Ambato, Ecuador.

Diego Carrion Galarza, MSc, Universidad Politécnica Salesiana, Ecuador.

Carmen Celi Sanchez, MSc, Universidad Politécnica Salesiana, Ecuador.

Diego Chacon Troya, MSc, Universidad Politécnica Salesiana, Ecuador.

Paul Chasi, MSc, Universidad Politécnica Salesiana, Ecuador.

JuAn Chica, MSc, Universidad Politécnica Salesiana, Ecuador.

Diego Marcelo Cordero Guzmán, MSc, Universidad Católica de Cuenca, Ecuador.

Luis Javier Cruz, PhD, Universidad Pontificia Bolivariana, Medellin, Colombia.

Fabricio Esteban Espinoza Molina, MSc, Universidad Politécnica Salesiana, Ecuador.

Jorge Fajardo Seminario, PhD, Universidad Politécnica Salesiana, Ecuador.

Patricia Fernandez Morales, PhD, Universidad Pontificia Bolivariana, Medellin, Colombia.

Marcelo Flores Vazquez, MSc, Universidad Politécnica Salesiana, Ecuador.

Carlos Flores Vázquez, MSc, Universidad Católica de Cuenca, Ecuador.

Carlos Franco Cardona, PhD, Universidad Nacional de Colombia, Colombia.

Cristian García García, MSc, Universidad Politécnica Salesiana, Ecuador.

Teonila García Zapata, PhD, Universidad Nacional Mayor de San Marcos, Perú.

Luis Garzón MÑOZ, PhD, Universidad Politécnica Salesiana, Ecuador.
Natalia Gonzalez Alvarez, MSc, Universidad Politécnica Salesiana, Ecuador.

Ernesto Granado, PhD, Universidad Simón Bolívar, Venezuela.

Adriana del Pilar Guaman, MSc, Universidad Politécnica Salesiana, Ecuador.

Juan Inga Ortega, MSc, Universidad Politécnica Salesiana, Ecuador.

Esteban Inga Ortega, PhD, Universidad Politécnica Salesiana, Ecuador.

PaOla Ingavélez, MSc, Universidad Politécnica Salesiana, Ecuador.

Cesar Isaza Roldan, PhD, Universidad Pontificia Bolivariana.

Nelson Jara Cobos, MSc, Universidad Politécnica Salesiana, Ecuador.

Ruben Jerves, MSc, Universidad Politécnica Salesiana, Ecuador.

Victor Ramon Leal, PhD, Investigador de PDVSA, Venezuela

Gabriel Leon, MSc, Universidad Politécnica Salesiana, Ecuador.

Edilberto Llanes, PhD, Universidad Internacional SEK, Ecuador.

Luis López López, PhD, Universidad Politécnica Salesiana, Ecuador.

Carlos Mafla Yépez, MSc, Universidad Técnica del Norte, Ecuador.

Hader Martínez, PhD, Universidad Pontificia Bolivariana, Medellin, Colombia

Javier Martínez, PhD, Instituto Nacional de Eficiencia Energética y Energías Renovables, Ecuador.

Alex Mayorga, MSc, Universidad Técnica de Ambato, Ecuador.

Jimmy Molina, MSc, Universidad Técnica de Machala, Ecuador.

Andres Montero, PhD, Universidad de Cuenca, Ecuador.

Vicente Morales, MSc, Universidad Tecnica de Ambato, Ecuador.

Fabián Morales, MSc, Universidad Técnica de Ambato, Ecuador.

Diego Morales, MSc, Ministerio de Electricidad y Energías Renovables del Ecuador.

Yoandrys Morales Tamayo, PhD, Universidad Técnica de Cotopaxi, Cotopaxi

Olena Leonidivna Naidiuk, MSc, Universidad Politécnica Salesiana, Ecuador.

Oscar Naranjo, MSc, Universidad del Azuay, Ecuador.

Paul Narvaez, MSc, Universidad Politécnica Salesiana, Ecuador.

Hernán Navas Olmedo, MSc, Universidad Técnica de Cotopaxi, Ecuador.

Cesar Nieto, PhD, Universidad Pontificia Bolivariana, Medellin, Colombia
Fabio Obando, MSc, Universidad Politécnica Salesiana, Ecuador

Luis Ortiz Fernandez, MSc, Universidade Federal de Rio Grande del Norte, Brasil

Pablo Parra, MSc, Universidad Politécnica Salesiana, Ecuador

Paulo Peña Toro, PhD, Ministerio de Productividad, Ecuador.

Patsy Prieto Velez, MSc, Universidad Politécnica Salesiana, Ecuador.

Diego Quinde Falconi, MSc, Universidad Politécnica Salesiana, Ecuador.

Diana Quintana Espinoza, MSc, Universidad Politécnica Salesiana, Ecuador.

William Quitiaquez Sarzosa, MSc, Universidad Politécnica Salesiana, Ecuador.

Flavio Quizhpi Palomeque, MSc, Universidad Politécnica Salesiana, Ecuador.

Washington Ramirez Montalvan, MSc, Universidad Politécnica Salesiana, Ecuador.

Fran Reinoso Avecillas, MSc, Universidad Politécnica Salesiana, Ecuador.

Néstor Rivera Campoverde, MSc, Universidad Politécnica Salesiana, Ecuador.

Jorge Romero Contreras, MSc, Universidad de Carabobo, Venezuela

Fabian SAenz Enderica, MSc, Universidad de las Fuerzas Armadas, ESPE, Ecuador.

Luisa Salazar Gil, PhD, Universidad Simón Bolivar, Venezuela

Gustavo Salgado Enríquez, Msc, Universidad Central del Ecuador., Ecuador.

Juan Carlos Santillán Lima, MSc, Universidad Nacional de Chimborazo

Andrés Sarmiento Cajamarca, MSc, Universidad Federal de Santa Catarina, Brasil

Rodolfo Bojorque Chasi, PhD, Universidad Politécnica Salesiana, Ecuador.

Paul Torres Jara, MSc, Universidad Politécnica Salesiana, Ecuador.

Rodrigo Tufiño CÁrdenas, MSc, Universidad Politécnica Salesiana, Ecuador.

Fernando Urgiles Ortíz, MSc, Universidad Politécnica Salesiana, Ecuador.

Juan Valladolid Quitoisaca, MSc, Universidad Politécnica Salesiana, Ecuador.

Mary Vergara Paredes, PhD, Universidad de los Andes, Merida, Venezuela

Jennifer Yepez Alulema, MSc, Universidad Politécnica Salesiana, Ecuador.

Julio Zambrano Abad, MSc, Universidad Politécnica Salesiana, Ecuador.

Patricia Zapata Molina, MSc, Universidad Politécnica Salesiana, Ecuador. 
Consejo de publicaciones

Juan Cárdenas Tapia, sdb, PhD

Javier Herrán Gómez, SDb, PhD

José Juncosa Blasco, PhD

Juan Pablo Salgado Guerrero, PhD

René Unda lara, PhD

Jaime Padilla Verdugo, PhD

Sheila Serrano Vincenti, MSc

Jorge Cueva Estrada, MSc

John Calle Sigüencia, PhD

Floralba Aguilar Gordón, PhD

Betty Rodas Soto, MSc

Mónica Ruiz VÁsquez, MSc

Paola Ingavélez Guerra, PhD

David Armendáriz González, MSc

Luis Álvarez Rodas, PhD

Editor General

Luis Álvarez-Rodas, PhD

\section{Consejo Técnico}

Dra. Marcia Peña, Revisora de Estilo, Centro Gráfico Salesiano - Editorial Don Bosco

Marlon Quinde Abril, MSc, Diagramación y Maquetación

Christian Sinchi Narvaez

Adriana Curiel Avila, Traductora

Ángel Torres-Toukoumidis, PhD

Servicio de Publicaciones

Hernán Hermosa (Coordinación General)

Marco Gutiérrez (Soporte OJS)

Paulina Torres (Edición)

RAYSA ANDRAdE (Maquetación)

Martha Vinueza (Maquetación)

\section{Editorial}

Editorial Abya Yala (Quito-Ecuador),

Avenida 12 de octubre N422 y Wilson,

Bloque A, UPS Quito, Ecuador.

Casilla 17-12-719 Teléfonos: (593-2) 3962800 ext. 2638

Correo electrónico: editorial@abyayala.org

Tiraje: 800 ejemplares

Sistema tipográfico usado en la composición de este documento $\mathrm{IT}_{\mathrm{E}} \mathrm{X}$. 


\section{INGENIUS}

\section{Revista de Ciencia y Tecnología}

Número 25, enero - junio 2021

ISSN impreso 1390-650X / ISSN electrónico 1390-860X

La administración de INGENIUS se realiza a través de los siguientes parámetros:

La revista utiliza el sistema antiplagio académico

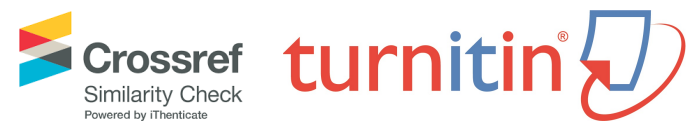

Los artículos cuentan con código de identificación (Digital Object Identifier)

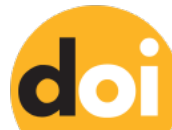

El proceso editorial se gestiona a través del Open Journal System

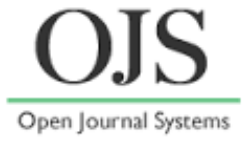

Es una publicación de acceso abierto (Open Access) con licencia Creative Commons

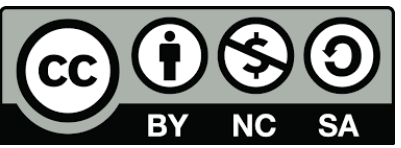

Las políticas copyright y de uso postprint, se encuentran publicadas en el Repositorio de Políticas de Autoarchivo Sherpa/Romeo.

\section{$\circlearrowleft$ SHERPA/ROMEO}

Los artículos de la presente edición pueden consultarse en http://revistas.ups.edu.ec/index.php/ingenius

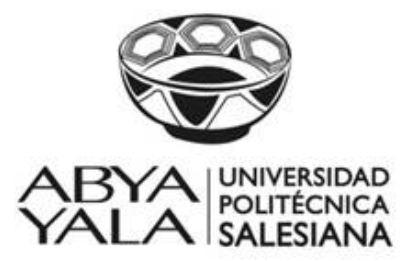

Universidad Politécnica Salesiana del Ecuador 
La Revista Ingenius está indexada en las siguientes Bases de Datos y sistemas de información científica:

BASE DE DATOS SELECTIVAS

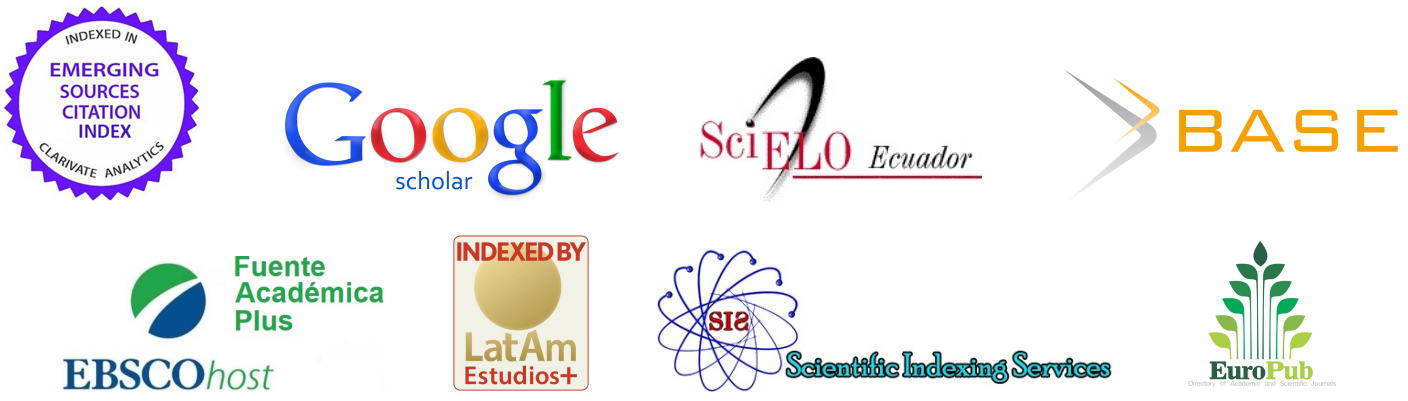

PLATAFORMAS DE EVALUACIÓN DE REVISTAS

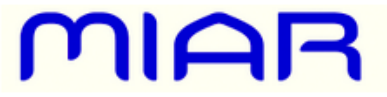

Oality Open Access Market

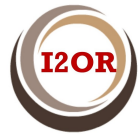

DIRECTORIOS SELECTIVOS
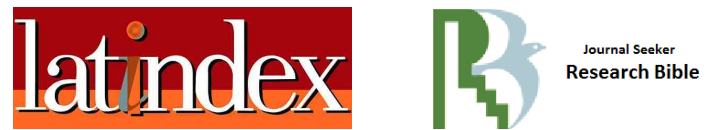

DAAcademickeys

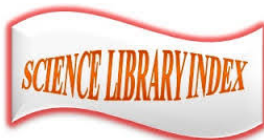

(D) ULRICHSWEB TM

HEMEROTECAS SELECTIVAS

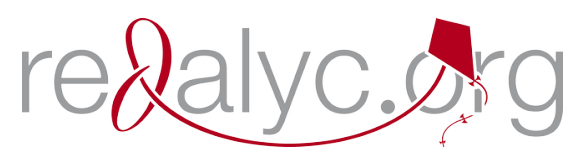

REDIB

refseek*

BUSCADORES DE LITERATURA CIENTÍFICA OPEN ACCESS
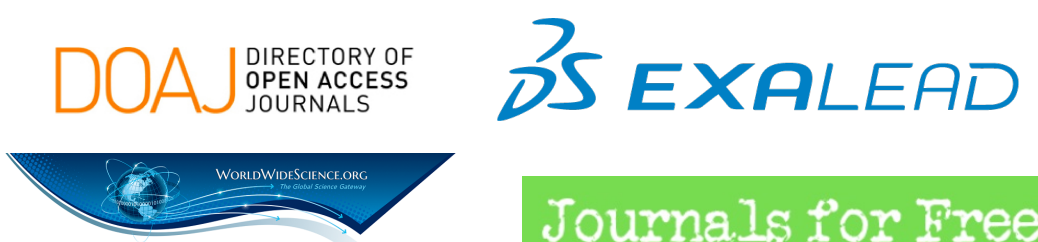

Journals for Firee

OTRAS BASES DE DATOS BIBLIOGRÁFICAS

D Dialnet louma PKP|INDEX 


\section{CATÁLOGO DE BIBLIOTECAS INTERNACIONALES UNIVERSITARIAS}

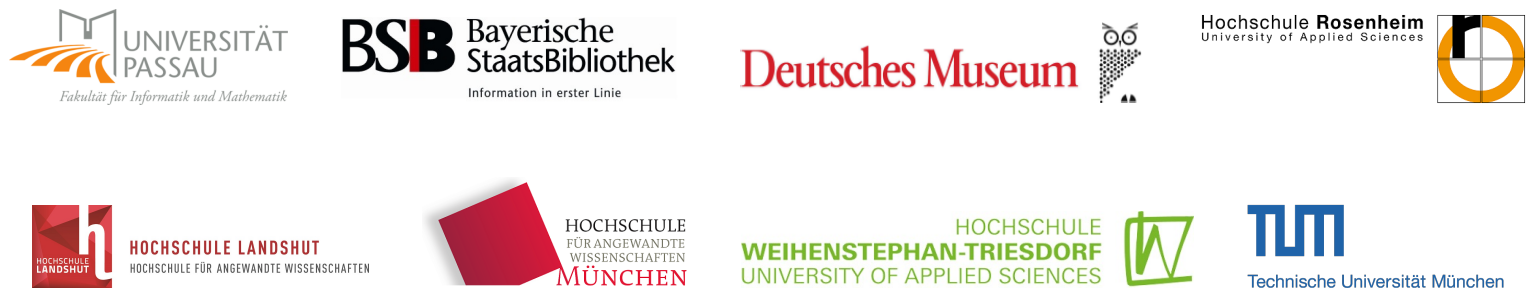

\begin{tabular}{|c|c|c|c|}
\hline Universität & LMU & $\overbrace{\substack{\text { Technische Hochschule } \\
\text { Ingolstadt }}}$ & $\cdot 1 \cdot L \cdot L \cdot \begin{array}{l}\text { Hochschule Augsburg } \\
\text { University of Applied Sciences }\end{array}$ \\
\hline
\end{tabular}

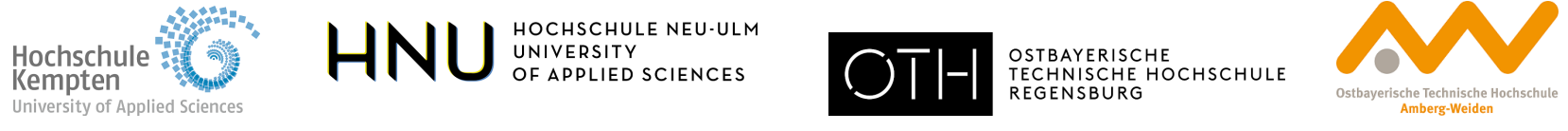

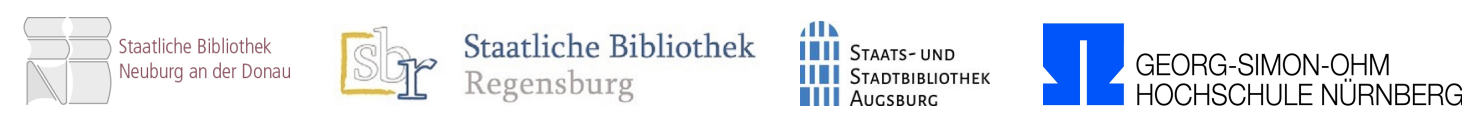

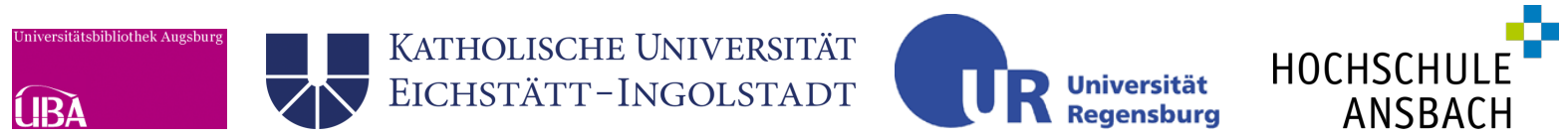

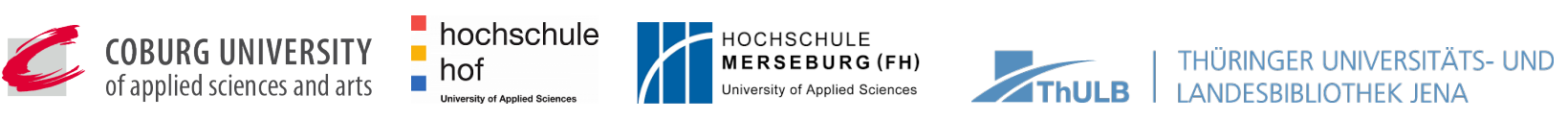

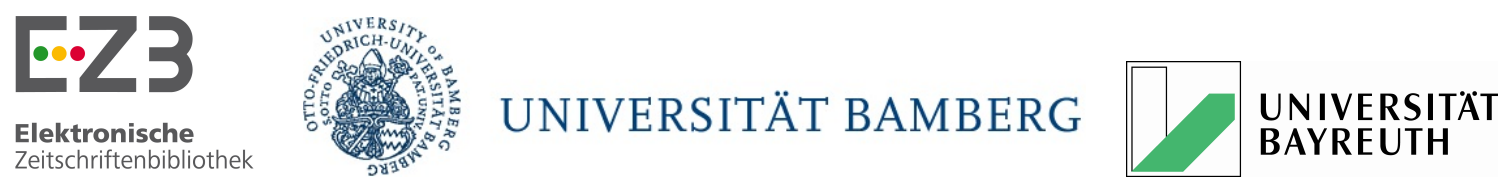

Zeitschriftenbibliothek

UNIVERSITÄT BAMBERG BAYREUTH
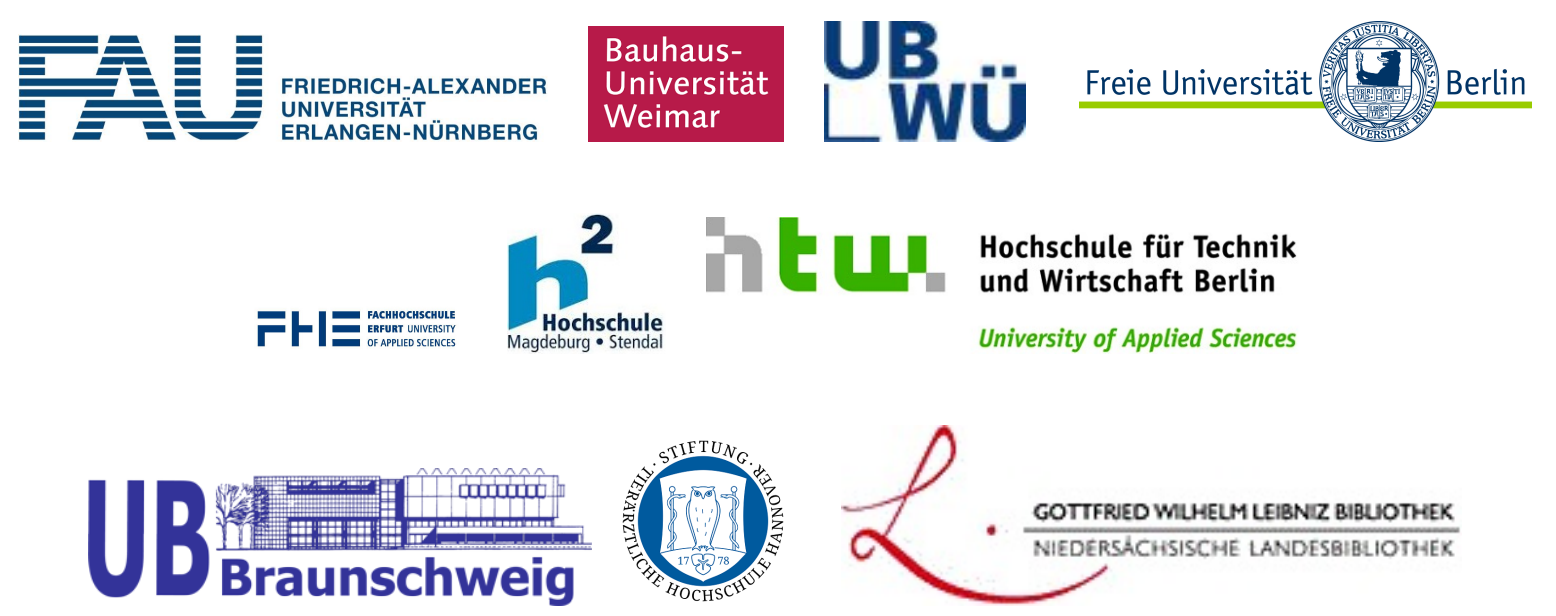


\section{EDITORIAL}

Estimadas y estimados lectores:

Los retos y desafíos que nos ha dejado el año 2020 nos hacen reflexionar acerca de lo importante que es la investigación para mantener literalmente con vida a la humanidad. La pandemia de la COVID-19 ha trastocado todos los ámbitos de nuestras existencias; aun esos emporios que se creían indestructibles se han visto afectados y muchos de ellos han requerido modificar sus actividades habituales para poder subsistir. Aquellos que no lo hicieron sucumbieron ante la crisis y cerraron sus puertas generando desempleo y aumentando la brecha entre los que tienen mucho y aquellos que deben ingeniárselas de alguna manera para sobrevivir.

Mantener activa la investigación no ha sido cosa fácil, las restricciones generadas en lo referente al acceso a los laboratorios, el libre tránsito de las personas, los procesos de importación de insumos, el riesgo que implica continuar con los procesos especialmente los que requieren experimentación con grupos y muchas cosas más son actualmente barreras que se deben superar. Dándole un rápido vistazo al gigantesco mundo de las publicaciones es evidente que estas no se han detenido, siguen generando sus contenidos periódicos con los índices de calidad establecidos. En el Ecuador, para quienes nos encontramos en la emocionante tarea de la difusión científica, esto es palpable y si consideramos la cantidad de artículos receptados en INGENIUS para este número, podemos entender que los investigadores idearon procesos para superar con éxito las barreras generadas por la pandemia.

Considero de suma importancia resaltar la capacidad de innovación e ingenio de los investigadores, es por esto por lo que la pandemia aunque ha provocado muchos problemas y pérdidas humanas, de seguro también ha generado grandes desafíos. El primero y que movió al trabajo en equipo de miles de investigadores de todo el mundo ha sido el desarrollo de una vacuna (conseguida en un tiempo récord); sin embargo, no olvidemos que se requerirá de una constante investigación para dar soporte a los diferentes cambios que sufra el virus y que modificará su interacción con los seres humanos. También hay que considerar que el bienestar de las personas se ha visto afectado y será necesaria una acción conjunta para recuperar y mejorar nuestros estilos de vida. Para esto, es primordial: generar propuestas y desarrollos para recuperar las fuentes de trabajo y mejorar la economía de todas las familias, aportando con ideas innovadoras que permitan optimizar la productividad, mejorando los sistemas de comunicación, fortaleciendo los sistemas de seguridad social, desarrollando tecnología de vanguardia, pero con costos accesibles que permitan a todas las personas ser parte de los beneficios que se obtengan. Y, sobre todo, aportando para que aquellos menos favorecidos tengan la oportunidad de integrarse a una vida digna y puedan desarrollarse para ser parte activa de la sociedad.

El trabajo en equipo se debe fortalecer y privilegiar, por lo que con mucho agrado hemos visto que varias de las contribuciones que llegan a INGENIUS están realizadas por grupos multidisciplinarios y de diferentes países, lo que ha dado lugar a artículos de gran valía que de seguro aportarán a la comunidad científica nacional e internacional en la búsqueda de productos, sistemas, equipos, procesos que permitan un desarrollo acelerado para conseguir la tranquilidad y el sosiego que esta pandemia nos quitó.

Finalmente, les invito a revisar los artículos seleccionados para esta edición: trabajos muy valiosos de diversas partes del mundo que, gracias a los sistemas virtuales y de comunicación, muestran que es posible alcanzar la sinergia necesaria para obtener resultados exitosos.

Y no olviden que si la investigación no continúa y se fortalece el mundo estará expuesto a una inminente extinción. 


\section{ÍNDICE}

Análisis del HFO-1234ze como alternativa ecológica en la refrigeración doméstica.... HFO-1234ze Analysis as an Ecological Alternative In Domestic Refrigeration

Cristian Andrade Terán

Impacto de los algoritmos de sobremuestreo en la clasificación de subtipos principales

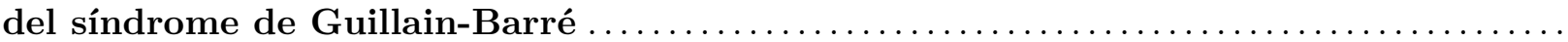

Impact of oversampling algorithms in the classification of Guillain-Barré syndrome main subtypes

Manuel Torres-Vásquez, José Hernández-Torruco, Betania Hernández-Ocaña, Oscar Chávez-Bosquez

Diseño y construcción de un reactor discontinuo con recirculación externa para obtener biodiésel a partir de aceite de fritura en condiciones subcríticas $\ldots \ldots \ldots \ldots \ldots \ldots \ldots$

Design and construction of a batch reactor with external recirculation to obtain biodisel from residual oil frying under subcritical conditions

Cristian Fabián Pérez-Salinas, Diego Fernando Núnez-Núñez, Herminia del Rosario Sanaguano-Salguero, Luis Fernando Sánchez-Quinchuela

Algoritmo de predicción del consumo de combustible para mezcla de etanol anhídrido

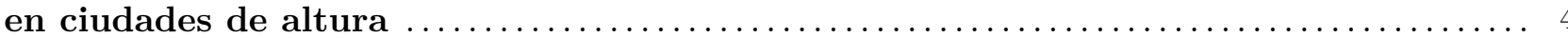

Prediction algorithm of fuel consumption for anhydrous ethanol mixture in high-altitude cities

Fabricio Espinoza, Fredy Tacuri, Wilmer Contreras, Javier Vázquez

Algoritmos para el reconocimiento de estructuras de tablas

Algorithms for Table Structure Recognition

Yosveni Escalona Escalona

Análisis de la eficiencia de un disco de freno convencional ventilado con respecto a un

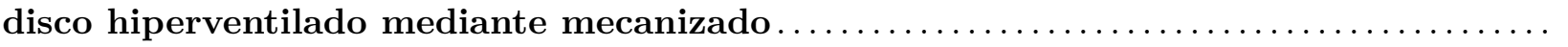

Analisys of the Efficiency of a Conventional Ventilated Brake Disc Compared to a Hyperventilated Disc by Machining

Vicente Rojas, Johnny Pancha, Vicente Romero, Jorge Lema

Evaluación de un radar FMCW como herramienta didáctica en las carreras de Inge-

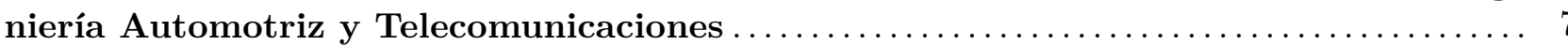

Evaluation of a FMCW radar as a teaching tool in the automotive and telecommunications engineering careers

Pablo J. Mavares F.

Simulación numérica del flujo subexpandido en la tobera cónica experimental helios-x 81 Numerical simulation of the under-expanded flow in the experimental conical nozzle helios- $\mathrm{x}$

San Luis B. Tolentino Masgo, Richard Nakka, Simón Caraballo, Jorge Mírez

Reduciendo la brecha de seguridad del IoT con una arquitectura de microservicios

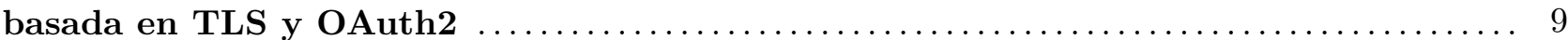

Reducing the IoT security breach with a microservice architecture based on TLS and OAuth2

Diego Ordóñez-Camacho

Análisis de vulnerabilidades con SQLMAP aplicada a entornos APEX 5

Vulnerability analysis with SQLMAP applied to APEX5 context

Esteban Crespo-Martínez

Normas editoriales

Guidelines 


\title{
ANÁLISIS DEL HFO-1234ZE COMO ALTERNATIVA ECOLÓGICA EN LA REFRIGERACIÓN DOMÉSTICA
}

\section{HFO-1234ZE ANALYSIS AS AN ECOLOGICAL Alternative In Domestic Refrigeration}

\author{
Cristian Andrade Terán ${ }^{1, *}$
}

Recibido: 03-05-2020, Revisado: 22-07-2020, Aprobado tras revisión: 05-08-2020

\section{Resumen}

La refrigeración de alimentos es un proceso esencial en los hogares, por lo que un refrigerador doméstico se convierte en un electrodoméstico indispensable. Siendo este uno de los mayores consumidores de energía eléctrica y de contaminación por el refrigerante que ocupa para su funcionamiento, es importante buscar alternativas que mejoren este proceso. En este estudio se pretende implementar un HFO, el R12354ze, como alternativa ecológica en la refrigeración doméstica, en respuesta a las demandas ambientales para reducir el cambio climático y el deterioro de la capa de ozono. Mediante un análisis termodinámico y de transferencia de calor con simulaciones del ciclo de refrigeración y el comportamiento del fluido en el intercambio de calor utilizando software especializado y CFD, se presenta al HFO como una alternativa aceptable logrando parámetros de refrigeración en rango entre 5\%-8 \% de diferencia con refrigeradores comunes utilizados actualmente, con un costo ambiental de hasta un $99 \%$ más bajo, sin alterar su eficiencia energética. Aprovechando las propiedades del HFO en el ciclo de refrigeración se logra mejorar el coeficiente de desempeño del ciclo de refrigeración en un $12 \%$.

Palabras clave: Acuerdo de París, CFD, HFO, enmienda Kigal, refrigeración, R1234ze

\section{Abstract}

Food refrigeration is an essential process in homes, and thus a home refrigerator becomes an indispensable appliance. Being this one of the biggest consumers of electrical energy and contamination due to the refrigerant used for its operation, it is important to look for alternatives that improve this process. This study aims to implement the HFO, R12354ze as an ecological alternative in domestic refrigeration, in response to environmental demands to reduce climate change and deterioration of the ozone layer. Through a thermodynamic and heat transfer analysis, simulating the cooling cycle and the behavior of the fluid in heat exchange using specialized software and CFD, the HFO is presented as an acceptable alternative achieving cooling parameters which are between $5 \%$ and $8 \%$ different from common refrigerators currently used, with an environmental cost up to $99 \%$ lower, without altering their energy efficiency. Taking advantage of the properties of the HFO, it is possible to improve the coefficient of performance of the cooling cycle by $12 \%$.

Keywords: CFD, HFO, Kigal amendment, Paris agreement, refrigeration, R1234ze.

\footnotetext{
1,* Ingeniería Mecánica, Universidad Politécnica Salesiana, Quito-Ecuador

Autor para correspondencia candradet@est.ups.edu.ec. (D) https://orcid.org/0000-0002- 0220-7481
}

Forma sugerida de citación: Andrade Terán, C. (2021). «Análisis del HFO-1234ze como alternativa ecológica en la refrigeración doméstica». IngEnius. N. ${ }^{\circ}$ 25, (enero-junio). pp. 9-19. DoI: https://doi.org/10.17163/ings.n25.2021.01. 


\section{Introducción}

La refrigeración mecánica que utiliza fluidos para la transferencia de calor, históricamente ha pasado por varias etapas para adaptarse a las necesidades ambientales. Comenzando con el Convenio de Viena para la protección de la capa de ozono (1985), que obligó a llegar al Protocolo de Montreal (1995) con respecto a sustancias que deterioran la misma (CFC y halógenos principalmente); este acuerdo fue exitoso y las metas propuestas fueron cumplidas. En 2003 se lo vio como el protocolo más exitoso hasta esa fecha; en 2015 se lo congeló y se espera para el 2030 eliminar por completo los HCFC en los países desarrollados y en el 2040 para los demás [1].

Consecuentemente las Naciones Unidas crean otros tratados y convenios para la protección de la capa de ozono y el medioambiente, entre estos destaca el Acuerdo de París en 2015 donde se firma la agenda 2030, se pide acelerar la reducción de las emisiones mundiales de gases de efecto invernadero, buscando mantener el aumento de la temperatura global en este siglo muy por debajo de los $2{ }^{\circ} \mathrm{C}$ [2]. En este contexto, en 2016, con la enmienda Kigali al Protocolo de Montreal, se llega a un acuerdo para eliminar gradualmente los hidrofluorocarbonos (HFC), estos a pesar de que tienen ODP nulo o bajo poseen un GWP alto (Global Warming Potential), lo que aumenta la temperatura del planeta. Esta enmienda entró en vigor el 1 de enero del 2019, para reducir la producción y el consumo de HFC en más de $80 \%$ durante los próximos treinta años [3].

Con la reunión en Kigali se acuerda reducir hasta aplacar el uso de refrigerantes con alto GWP, incentivando al cambio por alternativas menos perjudiciales al ambiente y mejorar la eficiencia energética en refrigeración y aire acondicionado. La oportunidad de cambio es muy aceptada y resulta en muchos casos rentable en refrigeración industrial y transporte refrigerado y en menor medida en la refrigeración comercial; sin embargo, al aire acondicionado móvil, comercial y refrigeración doméstica les resulta más costoso el cambio y, por ende, más difícil el alejarse de los HFC. Además, en países subdesarrollados y con alta temperatura ambiental resulta más costoso y difícil el cambio comparado con los países desarrollados [4]. Con las regulaciones y los impuestos a los refrigerantes con alto GWP se busca que sea más complicado para productores generar electrodomésticos con estos refrigerantes, haciendo que la industria y las investigaciones se centren en buscar alternativas más ecológicas, que no resulten en costos relativamente altos en la adaptación o remplazo en equipos de refrigeración y aire acondicionado que se han venido utilizando.

Los HFO, también llamados hidrofluoolefinas, podrían ser una de las principales alternativas para el campo de la refrigeración, actualmente son la cuarta generación de refrigerantes. En la Feria Internacional de Energías Verdes en Refrigeración y Aire Acondicionado, la ingeniera química Nohora Clavijo explicó que los HFO son compuestos orgánicos de doble enlace con una menor vida atmosférica, por lo que tienen menos impacto ambiental; además. la empresa Honeywell comunicó que se utilizarán mezclas de $\mathrm{HFO} / \mathrm{HFC}$ para reducir el uso de los hidrofluorocarbonados.

En análisis experimentales realizados para comparar HFO con HFC-134a en un sistema de compresión de vapor, la diferencia en el COP (coeficiente de desempeño) obtenido para R1234yf está entre el 3 y $11 \%$ menor al obtenido con R134a y para R1234ze está solamente entre el 2 y $8 \%$ por debajo, usando el mismo compresor para los tres casos [5]. Yataganbaba et al. [6] en una investigación de análisis de energía en un sistema de compresión de vapor, encontraron al R1234yf y al R1234ze como sustitutos adecuados para R134a, siendo este el refrigerante mayormente usado en refrigeración doméstica. Los HFO podrían trabajar similarmente en sistemas de refrigeración domésticos sin presentar la sanción ambiental del HFC.

\subsection{Bajos niveles de GWP y alta seguridad}

El GWP o potencial de calentamiento global que una sustancia química posee es producto de la combinación de su forzamiento radiativo o forzamiento climático (cambio en la irradiancia neta en la zona de transición entre la troposfera y la estratosfera debido al cambio en la concentración atmosférica de un gas) y la vida útil atmosférica (tiempo que permanece sin desintegrarse en la atmósfera) [7].

$\mathrm{Al}$ ser las hidrofluoroolefinas compuestos fluorados sin carbono, presentan niveles muy bajos de GWP y un ODP nulo, cumpliendo con las normas establecidas para proteger la capa de ozono y el ambiente. Además, los HFO, a diferencia de los hidrocarburos (HC), no presentan riesgo alto de inflamabilidad, con clasificación ASHRAE de seguridad entre A1 y A2, inflamabilidad baja o nula.

Otra gran ventaja de estos refrigerantes es que son compatibles con gran variedad de aceites lubricantes y no es necesario hacer adaptaciones importantes en los sistemas existentes que funcionan con HFC o HCFC. No causa desgaste en los materiales por corrosión y por sus propiedades no ocasiona elevada fatiga en el compresor.

\subsection{Implementación de R1234ze a la refrigeración doméstica}

La mayoría de refrigeradores domésticos por compresión de vapor utilizan HFC como fluido de trabajo en el ciclo, estos no dañan directamente a la capa de ozono, pero sí contribuyen al efecto invernadero, el más usado es el R134a. También se está utilizando como 
sustitutos ideales a hidrocarburos para la refrigeración doméstica, productos como el R600a, que presenta muy bajo aporte al calentamiento global; sin embargo, son altamente inflamables si no se los trata debidamente y es necesario el cambio en la instalación total. Por el contrario, el HFO-1234ze necesita diez veces más concentración y 250000 veces más energía que los hidrocarburos para inflamarse, solo por encima de $30{ }^{\circ} \mathrm{C}$. Está catalogado como fluido de baja toxicidad y ligeramente inflamable [8-10].

El refrigerante (HFO) R1234ze presenta características de eficiencia muy similares al HFC R134a y trabaja a presiones parecidas por lo que no es necesario cambios importantes en los sistemas ya existentes. La vida atmosférica del refrigerante como residuo es de solo dieciocho días, mucho más baja que los trece años del R134a. Una característica única del R1234ze es la ausencia de inflamabilidad al mezclarse con aire a menos de $30{ }^{\circ} \mathrm{C}$ de temperatura ambiente. Por eso se considera no inflamable para manipulación y almacenamiento [11], [12]. Ngoc [13] en su investigación de un ciclo de refrigeración que utiliza HFO-1234ze, HFO-1234yf, R22 y R32 como refrigerantes alternativos a R134a; los resultados muestran que el ciclo que usa R1234ze como refrigerante tiene el coeficiente de rendimiento más alto.

Debido a estas compatibilidades y características, un sistema de refrigeración de presión media o baja, en este caso un refrigerador doméstico convencional, puede ser acoplado a trabajar con R1234ze sin ser necesario el realizar cambios importantes al sistema.

\section{Materiales y métodos}

El ciclo de refrigeración que trabajará con R1234ze en el estudio, se lo diseña según los datos obtenidos de un refrigerador doméstico funcional que trabaja con R134a. Se utiliza Genetron Properties, software proporcionado por Honeywell y la base de datos del laboratorio de Investigación de Buffalo - NY-USA para la modelación de este ciclo termodinámico. Además, para el análisis de transferencia de calor del refrigerante en el ciclo de refrigeración se realiza el estudio CFD utilizando Fluent de ANSYS, en el cual se realiza pruebas de evaporación en el respectivo dispositivo y de distribución de temperaturas en el electrodoméstico, simulando las condiciones reales en las que trabajaría.

\subsection{Características del refrigerante}

El HFO 1234ze es un fluido puro con baja toxicidad e inflamabilidad, formado por moléculas de flúor, hidrógeno y oxígeno. Las propiedades de este fluido se presentan en la Tabla 1 a manera de comparación con el refrigerante 134a.
Tabla 1. Propiedades de R134a y R1234ze

\begin{tabular}{ccc}
\hline Propiedad & R134a & R1234ze \\
\hline Peso molecular $(\mathrm{kg} / \mathrm{kmol})$ & 102 & 114 \\
Temp. crítica & 101,1 & 109,4 \\
$\left({ }^{\circ} \mathrm{C}\right)$ & & \\
Densidad & 511,9 & 489,23 \\
$\left(\mathrm{~kg} / \mathrm{m}^{3}\right)$ & & \\
Presión crítica & 4059 & 3636 \\
$(\mathrm{kPa})$ & 0 & 0 \\
ODP & 1300 & 1 \\
GWP & A1 & A2L \\
\hline
\end{tabular}

Datos tomados del catálogo de Honeywell [12] y REFPROP [14]

\subsection{Ciclo de refrigeración}

Para el estudio del ciclo de refrigeración se toman las condiciones de ciclo en un refrigerador con R134a para clima subtropical que tiene una potencia nominal de $0,104 \mathrm{~kW}$ y trabaja con un compresor Embraco EM3U50HLP de 1/5 de hp. En la Tabla 2 se presenta las características del refrigerador de referencia.

Tabla 2. Características del refrigerador referencial

\begin{tabular}{cc}
\hline Característica & Valor \\
\hline Denominación & Refrigerador doméstico \\
sin escarcha \\
Tipo de refrigerante & $\mathrm{R} 134 \mathrm{a}$ \\
Masa de refrigerante & $95 \mathrm{~kg}$ \\
Potencia nominal & $104 \mathrm{~W}$ \\
Volumen nominal & $250 \mathrm{l}$ \\
Volumen congelador & $55 \mathrm{l}$ \\
Volumen refrigerador & $184 \mathrm{l}$ \\
\hline
\end{tabular}

El refrigerador funciona con temperatura de evaporación a $-23,3{ }^{\circ} \mathrm{C}$ y condensación a $54,4{ }^{\circ} \mathrm{C}$, de acuerdo con estas condiciones se elabora el ciclo termodinámico para R134a. Los parámetros del ciclo se definen mediante las propiedades termodinámicas propias del refrigerante en las condiciones de funcionamiento en el refrigerador. En la Figura 1 se muestra el diagrama P-h del ciclo de refrigeración con R134a que se realizó utilizando EES [15] y los datos obtenidos experimentalmente.

En el cálculo de los parámetros fundamentales de desempeño del ciclo de refrigeración, se obtiene el coeficiente de desempeño (COP) con la Ecuación 1, este parámetro representa la relación de la capacidad de enfriar con el costo energético causado por el ciclo en la compresión.

$$
C O P=\frac{Q_{e}}{W_{c}}
$$




$$
Q_{e}=\left(h_{1}-h_{4}\right) \dot{m}
$$

Donde:

$Q_{e}$ : Capacidad frigorífica o de enfriamiento, $[\mathrm{W}]$

$W_{c}$ : Potencia consumida, [W]

$h_{1}$ : Entalpía salida del evaporador, $[\mathrm{kJ} / \mathrm{kg}]$

$h_{4}$ : Entalpía entrada del evaporador, $[\mathrm{kJ} / \mathrm{kg}]$

$\dot{m}$ : Flujo másico de refrigerante, $[\mathrm{kg} / \mathrm{s}]$

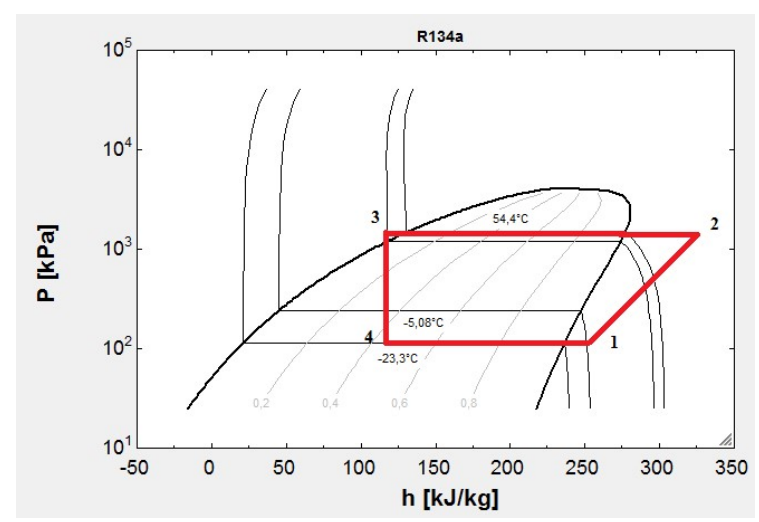

Figura 1. Diagrama P-h, ciclo de refrigeración real con R134a [15]

La eficiencia isentrópica del compresor es la generación de entropía en la compresión real comparada con la generada idealmente. Este parámetro se define por la Ecuación 3.

$$
\eta=\frac{h_{2 s}-h_{1}}{h_{2 a}-h_{1}}
$$

Donde:

$\eta$ : eficiencia isentrópica

$h_{2 s}$ : Capacidad frigorífica, $[\mathrm{kJ} / \mathrm{kg}]$

$h_{2 a}$ : Potencia consumida, $[\mathrm{kJ} / \mathrm{kg}]$

\subsection{Ciclo de refrigeración adaptado a R1234ze}

Para la comparación de desempeño de R1234ze con R134a se adapta teóricamente el ciclo del refrigerador doméstico de referencia a operar con HFO, sin alterar las condiciones de trabajo. Para esto se diseña el ciclo de refrigeración, utilizando Genetron Properties, se mantiene los parámetros originales del refrigerador de estudio, se ingresa la potencia de consumo, temperatura de condensación, temperatura de evaporación y eficiencia del compresor. Esta simulación será llamada caso A. En la Figura 2 y en la Figura 3 se presentan los diagramas P-h y T-s para el ciclo de refrigeración con R1234ze.

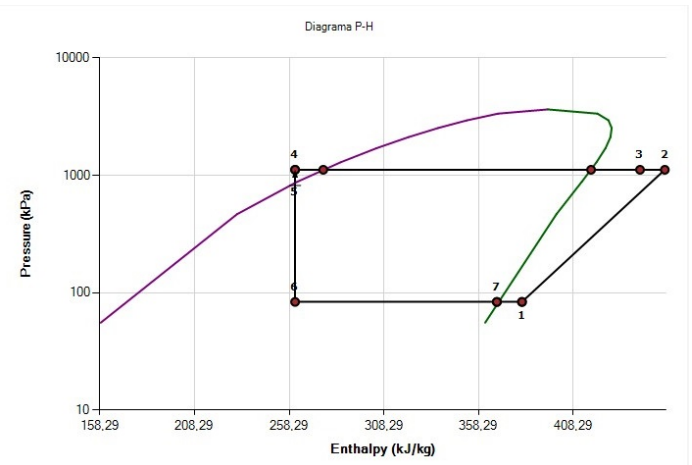

Figura 2. Diagrama P-h, ciclo de refrigeración real con R1234ze

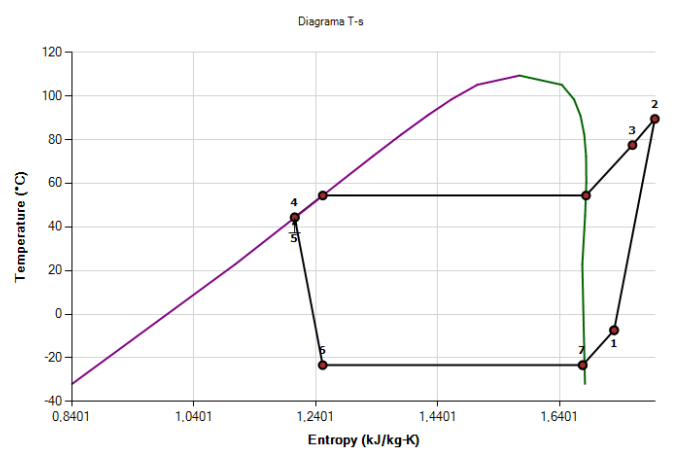

Figura 3. Diagrama T-s, ciclo de refrigeración real con R1234ze

\subsubsection{Mejoras al ciclo termodinámico}

De acuerdo con las indicaciones de los proveedores de R1234ze se podría mejorar el COP del ciclo compensando la capacidad de enfriamiento del sistema con ciertos ajustes en él, además, al mejorar el rendimiento del compresor se incrementaría la eficiencia del refrigerador con el HFO. La calidad de la mezcla a la salida del evaporador puede ser mejor con R1234ze que con R134a, si el flujo de calor es suficientemente bajo o la velocidad de la masa es suficientemente alta [16], además, se ha visto experimentalmente que el COP mejora para ciclos con R1234ze a mayores valores de temperatura de evaporación [5]. Por otro parte, Sánchez et al. [17] indican que se debe usar un compresor con mayor desplazamiento para alcanzar la misma capacidad de enfriamiento que con un sistema con R134a.

Se propone un ciclo mejorado, donde se realizan algunas modificaciones para que el desempeño del ciclo de refrigeración mejore. Se modifican parámetros de funcionamiento del compresor, se reajustan las temperaturas de condensación y evaporación acomodando las presiones de trabajo, se mantienen las características físicas del sistema, así como la potencia de suministro. Cabe recalcar que las modificaciones son teóricas y 
para funcionamiento experimental se necesitaría cambios físicos a la instalación del refrigerador doméstico. Sin embargo, estos arreglos son menores comparados con los necesarios para implementar hidrocarburos [10].

En la Figura 4 se presenta el diagrama termodinámico del ciclo de refrigeración para los casos estudiados. En el caso B se mejora el rendimiento del compresor, aumentando el desplazamiento volumétrico y su eficiencia isentrópica. En el caso $\mathrm{C}$ se modifican las temperaturas de trabajo, elevando la temperatura de evaporación $5,3^{\circ} \mathrm{C}$ y reduciendo la temperatura de condensación $4,4{ }^{\circ} \mathrm{C}$. Todas estas modificaciones buscan mejorar el rendimiento y desempeño del refrigerante en el ciclo de termodinámico de refrigeración.

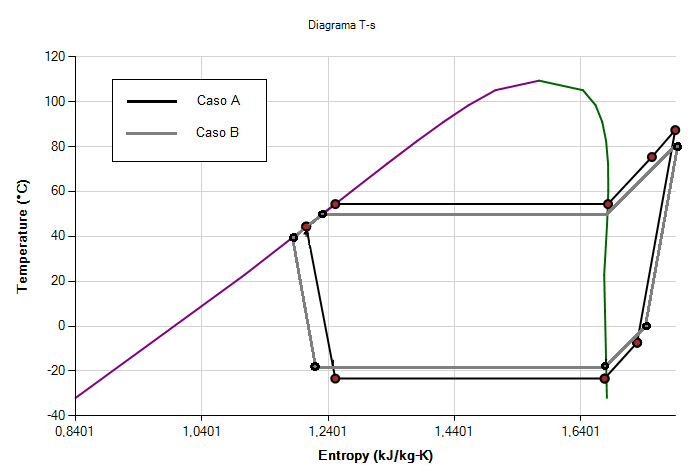

Figura 4. Mejoras al ciclo de refrigeración real con R1234ze Caso A y Caso B, diagrama T-s

\subsection{Estudio CFD de transferencia de calor}

En el estudio CFD se analiza lo que sucede con el refrigerante en el intercambiador de calor que realiza la absorción del calor, simulando el paso del refrigerante por el evaporador para obtener datos sobre el cambio de fase, temperatura y comportamiento del refrigerante R1234ze. Además, se simula el flujo de aire en el refrigerador con la capacidad de enfriamiento que aporta el HFO, para comparar con las temperaturas alcanzadas en los compartimentos con el R134a las cuales son $-13{ }^{\circ} \mathrm{C}$ para el congelador y $7{ }^{\circ} \mathrm{C}$ para el refrigerador, con un funcionamiento promedio a una temperatura ambiente de $18{ }^{\circ} \mathrm{C}$.

Se realiza el estudio en un modelo geométrico con las dimensiones del refrigerador de referencia que trabaja con R134a. El modelo tiene un volumen total de 2391 de espacio de contenedor de servicio, entre congelador y enfriador. El evaporador está compuesto por tubería de cobre tipo $\mathrm{K}$ en forma de serpentín, con diámetro nominal de $3 / 8$ pulgada ubicado en la zona del congelador. La geometría del volumen de control para el refrigerante en el evaporador y el volumen de control para el aire dentro del refrigerador se muestran en la Figura 5.

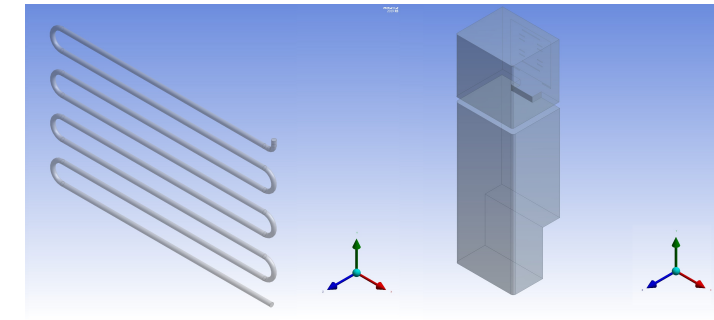

Figura 5. Geometría del volumen de control para simulación. a) Refrigerante en el evaporador. b) Aire dentro del refrigerador

\subsubsection{Modelos matemáticos para simulación}

Para el aire circundante dentro del refrigerador se utiliza el modelo volumen de fluido para simular la circulación de aire y el cambio de temperatura. Se utiliza el enfoque Euler-Euler para el modelo multifase en el evaporador, este resuelve ecuaciones de momento y continuidad. Se modelan dos fases diferentes que interactúan en el cambio de fase, la primera como líquido o mezcla líquido-vapor y la segunda fase como vapor. Utilizando el concepto de fracción de volumen con ecuaciones de régimen para cada fase, para la concentración de masa en la interacción de las fases se utiliza el modelo establecido por la Ecuación 4.

$$
A_{i}=\frac{6 \alpha_{p}\left(1-\alpha_{p}\right)}{d_{p}}
$$

Donde:

$A_{i}$ : Área interfacial

$\alpha_{p}$ : Fracción de volumen fase $q$

$d_{p}$ : Diámetro de burbuja

Los efectos de la viscosidad y turbulencia en el fluido se simulan con el modelo $k-\varepsilon \mathrm{RNG}$, que incluye el efecto de remolino en la turbulencia y se mejora el efecto del número de Reynolds para la viscosidad efectiva [18]. El modelo cuenta con expresiones para cada variable, Ecuación 5 y Ecuación 6.

Para $k$ :

$$
\begin{gathered}
\frac{\partial}{\partial r}(p k)+\frac{\partial}{\partial x i}\left(p k u_{i}\right)= \\
\frac{\partial}{\partial x j}\left(\alpha_{k} \mu_{e f f} \frac{\partial}{\partial x j}\right)+G_{k}+G_{b}-\rho \varepsilon-Y_{M}+S_{k}
\end{gathered}
$$

Para $\varepsilon$ :

$$
\begin{gathered}
\frac{\partial}{\partial r}(p \varepsilon)+\frac{\partial}{\partial x i}\left(p \varepsilon u_{i}\right)= \\
\frac{\partial}{\partial x j}\left(\alpha_{\varepsilon} \mu_{e f f} \frac{\partial}{\partial x j}\right)+C_{1 \varepsilon} \frac{\varepsilon}{k}\left(G_{k}+C_{3 s} G_{b}\right)- \\
-C_{2 \varepsilon} \rho \frac{\varepsilon^{2}}{k}-R_{\varepsilon}+S_{\varepsilon}
\end{gathered}
$$


Donde:

$G_{k \text { : }}$ Generación de energía cinética de turbulencia debido a la velocidad media

$G_{b}$ : Generación de energía cinética de turbulencia debido a la flotabilidad

$Y_{M}$ : Contribución de la dilatación fluctuante en la turbulencia compresible a la tasa de disipación general

$S$ y $C$ : Constantes y términos definidos para cada variable

$R \varepsilon$ : Término que se agrega en el método RNG para aumentar la precisión

$\alpha k$ y $\alpha \varepsilon$ : Inversos de los números de Prandtl efectivo para $k$ y $\varepsilon$, respectivamente

$\mu_{\text {eff }}$ : Viscosidad efectiva

La conservación de energía y de masa se establecen mediante balance de la cantidad de materia que entra y la cantidad que sale, bajo el modelo de Euler las leyes que rigen estos parámetros están definidas con la Ecuación 7 y con la Ecuación 8, respectivamente.

Conservación de energía:

$$
\begin{array}{r}
\frac{\partial}{\partial_{t}}\left(\alpha_{q} \rho_{q} h_{q}\right)+\nabla \cdot\left(\alpha_{q} \rho_{q} \vec{u}_{q} h_{q}\right) \\
=-\alpha_{q} \frac{\partial p_{q}}{\partial t}+\overline{\bar{t}}_{q}: \nabla \vec{u}_{q}-\nabla \cdot \vec{q}_{q}+S_{q}+ \\
+\sum_{p=1}^{n}\left(Q_{p q}+\dot{m}_{p q} h_{p q}-\dot{m}_{q p} h_{q p}\right)
\end{array}
$$

Conservación de masa:

$$
\begin{array}{r}
\frac{\partial}{\partial_{t}}\left(\alpha_{q} \rho_{q}\right)+\nabla \cdot\left(\alpha_{q} \rho_{q} \vec{u}_{q}\right)= \\
\sum_{p=1}^{n}\left(\dot{m}_{p q}+\dot{m}_{q p}\right)+S_{q}
\end{array}
$$

Donde:

$h_{q}$ : Entalpía específica para la fase secundaria

$Q_{p q}$ : Intensidad de intercambio de calor entre la fase primaria y la secundaria

$h_{p q}$ : Entalpía de cambio de fase

$\vec{v}_{q}$ : Velocidad de la fase secundaria $q$

$\dot{m}_{p q}$ : Masa transferida de la fase primaria a la secundaria

$\dot{m}_{q p}$ : Masa transferida de la fase secundaria a la primaria

El modelo de Lee define la transferencia de masa por condensación o evaporación de una fase a otra, se define la fase líquido como la fase primaria y la fase vapor como secundaria dependiendo de las condiciones del refrigerante al entrar al intercambiador, este modelo está descrito por la Ecuación 9.

$$
\frac{\partial}{\partial_{t}}\left(\alpha_{p} \rho_{p}\right)+\nabla \cdot\left(\alpha_{p} \rho_{p} \vec{V}_{p}\right) \dot{m}_{q p}-\dot{m}_{p q}
$$

Donde:

$\rho_{p}$ : Fase de vapor

$\vec{V}_{p}$ : Velocidad del vapor

$\dot{m}_{p q}$ : Tasa de masa transferida en evaporación

$\dot{m}_{q p}$ : Tasa de masa transferida en condensación

Además, en la interacción entre fases se integra la correlación de Moraga para definir el coeficiente Lift para simular los efectos que se producen por el remolino causado por el paso del fluido en forma de líquido, el coeficiente de arrastre se define por la correlación Schiller-Naumann para simular el arrastre en la superficie del fluido entre fases [18].

\section{Resultados y discusión}

Los parámetros termodinámicos obtenidos en el ciclo del refrigerador doméstico con R134a, usados como base para el ciclo con R124ze, están presentados en la Tabla 3.

Tabla 3. Parámetros del ciclo de refrigeración con R134a

\begin{tabular}{cc}
\hline \hline Parámetro & $\mathrm{R} 134 \mathrm{a}$ \\
\hline Temp. de evaporación & $-23,3{ }^{\circ} \mathrm{C}$ \\
Sobrecalentamiento & $18,3{ }^{\circ} \mathrm{C}$ \\
Temp. de condensación & $54,4{ }^{\circ} \mathrm{C}$ \\
Subenfriamiento & $8^{\circ} \mathrm{C}$ \\
Flujo másico & $0,0012 \mathrm{~kg} / \mathrm{s}$ \\
Eficiencia isentrópica & 0,688 \\
Potencia consumida & $0,104 \mathrm{~kW}$ \\
\hline
\end{tabular}

Con las pruebas termodinámicas realizadas en las simulaciones para R1234ze y los datos obtenidos para R134a se puede observar que la diferencia en el coeficiente de desempeño (COP) del ciclo con R1234ze es reducida en comparación con el de R134a; en la Figura 6 se presentan estos resultados.

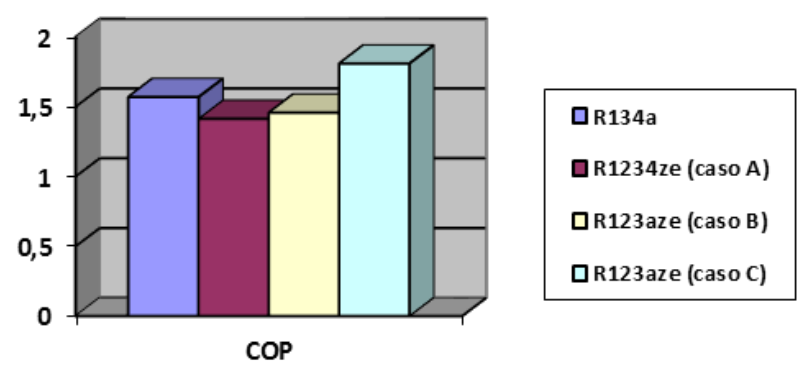

Figura 6. Coeficiente de desempeño (COP) 
Lo más importante en un sistema de refrigeración es la capacidad de producir frío o la de eliminar calor, para esto se calcula la capacidad de enfriamiento y la capacidad de calefacción que presenta el ciclo de refrigeración. En la Figura 7 se comparan estos parámetros del ciclo con R134a y los casos A, B y C para R1234ze.

\section{Capacidad calorífica [W]}

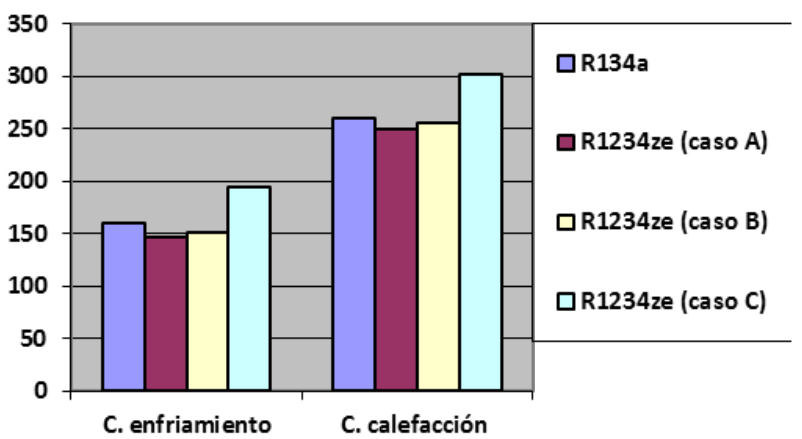

Figura 7. Capacidad de enfriamiento y de calefacción en los ciclos termodinámicos

Se puede observar que el caso C presenta la más alta capacidad calorífica debido a las modificaciones realizadas en las temperaturas de trabajo; sin embargo, para realizar esta modificación es necesario un mayor consumo de energía por parte del compresor lo que disminuiría la eficiencia energética del refrigerador. Por otra parte, los casos A y B presentan capacidades muy similares al ciclo con R134a.

Otro parámetro donde se encontró importantes cambios en el estudio termodinámico es la temperatura de descarga del compresor. Este dato es útil debido a que una elevada temperatura de descarga se traduce en alta fatiga para el compresor, además de no ser favorable en el ciclo termodinámico al aumentar el trabajo consumido por el compresor. En la Figura 8 se presentan los datos obtenidos de variación de temperatura en el compresor para los dos refrigerantes.

Temperaturas durante la compresión $\left[{ }^{\circ} \mathrm{C}\right]$

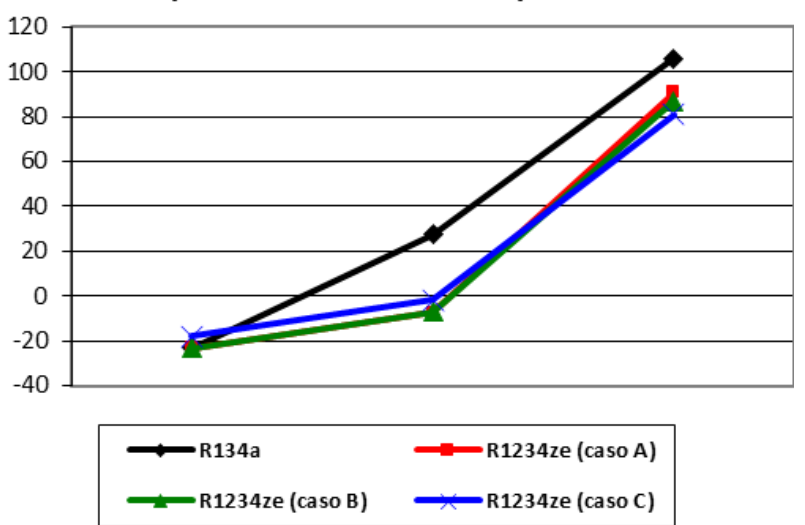

Figura 8. Variación de la temperatura en el proceso de compresión
Debido a las propiedades del HFO se genera menos fatiga en el compresor debido a una menor temperatura de descarga, además, es altamente compatible con varios materiales a diferencia de los hidrocarburos; esto marca una gran ventaja para los HFO en ciclos de compresión de vapor. Un factor importante para el desempeño del compresor también es la velocidad con la que debe fluir el refrigerante. En la Tabla 4 se presenta el flujo másico requerido para cada ciclo estudiado.

Tabla 4. Flujo másico requerido para cada ciclo $[\mathrm{kg} / \mathrm{s}]$

\begin{tabular}{cccc}
\hline R134a & R1234ze (A) & R1234ze (B) & R1234ze (C) \\
\hline 0,0012 & 0,0013 & 0,0014 & 0,0016 \\
\hline
\end{tabular}

\subsection{Resultados estudio CFD}

\subsubsection{Refrigerante en el evaporador}

En un ciclo de refrigeración por compresión de vapor, el evaporador es el encargado de la absorción del calor, de transportarlo hacia el compresor y luego en el condensador expulsarlo al ambiente. En este proceso de absorción, el refrigerante experimenta un cambio de fase de mezcla líquido-vapor a vapor y el desempeño que presente el refrigerante en el proceso es uno de los principales factores que determinan la capacidad de enfriamiento del ciclo.

En la Figura 9 se presenta el cambio de fase a lo largo del evaporador para el refrigerante R1234ze para constatar que el HFO cumple con la evaporación completamente en el intercambiador y no entrega vapor húmedo al compresor, lo que podría causar deterioro en él o mal funcionamiento en la compresión. Se compara el comportamiento con el refrigerante R134a, teniendo una escala de 0 a 1 para la calidad de mezcla, siendo 0 para el estado de ingreso (0,46 para R1234ze y 0,44 para R134a) al evaporador como mezcla líquido-vapor y 1 vapor saturado.

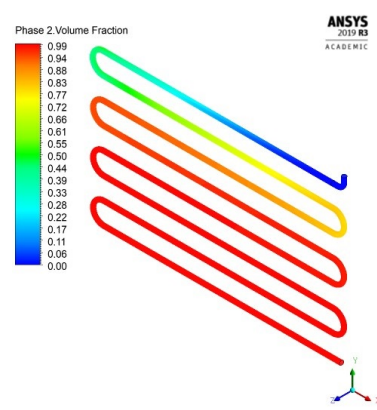

(a)

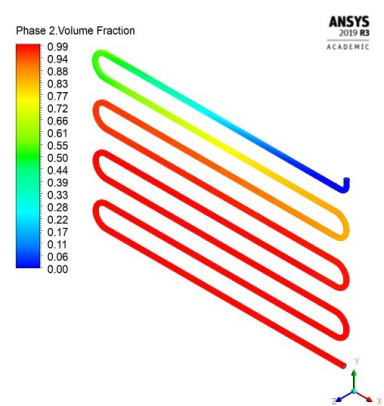

(b)
Figura 9. Estado del refrigerante durante el cambio de fase en el evaporador. a) R1234ze, b) R134a

Las características del refrigerante a la entrada del evaporador, tales como presión de trabajo, calidad y 
flujo másico, están dadas por el ciclo termodinámico descrito anteriormente para cada refrigerante. Se puede observar que en estas condiciones la evaporación es similar para los dos refrigerantes comparados, el R134a necesita menos longitud de tubería para completar el cambio de fase; sin embargo, el HFO entrega vapor sobrecalentado en mayor porcentaje. En la Figura 10 se muestra un contorno de área promedio medida en la salida del evaporador con la calidad total de la mezcla.

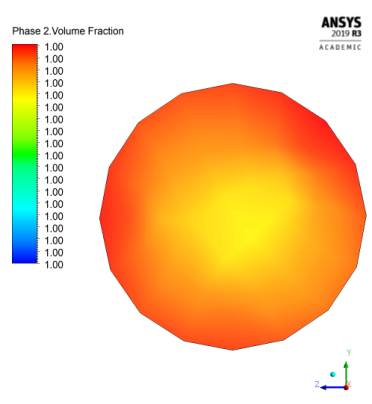

(a)

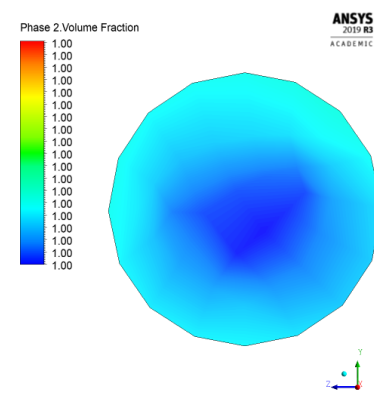

(b)
Figura 10. Calidad del refrigerante en la salida del evaporador. a) R1234ze, b) R134a

También se obtiene valores de la temperatura del refrigerante al salir del evaporador, Figura 11. En los resultados se observa que existe sobrecalentamiento durante la absorción del calor, la temperatura es mayor en la superficie del fluido en contacto con la pared del evaporador, llegando a alcanzar en promedio $-6,83^{\circ} \mathrm{C}$ y en en el interior del fluido se mantiene en la temperatura de saturación de $-23,3{ }^{\circ} \mathrm{C}$.

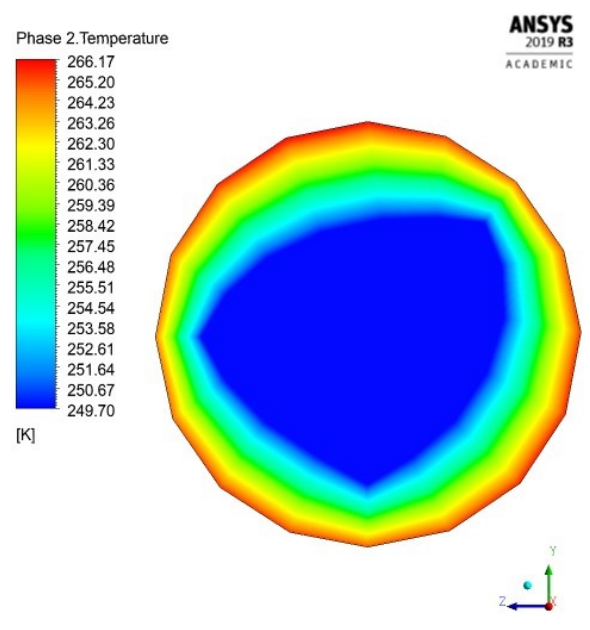

Figura 11. Temperatura del refrigerante (R1234ze) en la salida del evaporador

En comparación con el HFC-134a, el HFO-1234ze alcanza una mayor temperatura de sobrecalentamiento al terminar el proceso de evaporación, esta variación es muy baja por lo que no causaría un efecto importante en la entrada al compresor. En la Figura 12 se realiza una comparación de la temperatura tomada en varios puntos del fluido al dejar el evaporador.

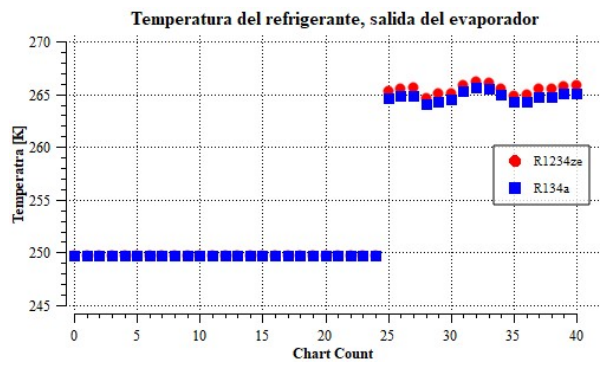

Figura 12. Comparación de temperatura de sobrecalentamiento

Los resultados obtenidos con el estudio CFD de transferencia de calor concuerdan con los resultados hallados con el análisis termodinámico realizado en el evaporador con R1234ze, teniendo los datos para cada estudio respectivamente en la Tabla 5 .

Tabla 5. Resultados estudios para el evaporador

\begin{tabular}{ccc}
\hline Parámetro & CFD & $\begin{array}{c}\text { Estudio } \\
\text { termodinámico }\end{array}$ \\
\hline T. evaporación $\left[{ }^{\circ} \mathrm{C}\right]$ & $-23,3$ & $-23,3$ \\
T. sobrecalentamiento $\left[{ }^{\circ} \mathrm{C}\right]$ & $-6,83$ & $-7,3$ \\
Flujo másico $[\mathrm{kg} / \mathrm{s}]$ & 0,0013 & 0,0013 \\
Calidad del fluido & 1 & 1 \\
C. de enfriamiento $[\mathrm{W}]$ & 146,3 & 146,4 \\
\hline
\end{tabular}

\subsubsection{Flujo de aire dentro del refrigerador}

Para el flujo de aire en el volumen de control de 239 1 total en los compartimentos, con 55 l para el congelador y $184 \mathrm{l}$ en el enfriador, se simula la circulación de aire a una velocidad promedio de 1,103 m/s generada por un ventilador de refrigerador doméstico no-frost que trabaja a 2070 RPM [18]. En la Figura 13 se esquematiza el flujo de aire dentro del refrigerador.

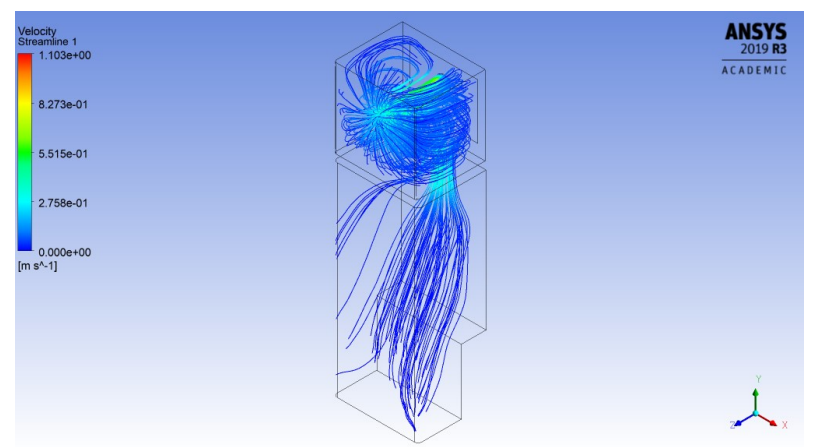

Figura 13. Flujo de aire en el refrigerador

Se realizaron pruebas con este flujo para las capacidades de enfriamiento obtenidas en cada ciclo de 
refrigeración con R1234ze en los casos A, B y C de estudio. En la Figura 14 se presentan los resultados en un volume rendering de la temperatura en el aire, obtenida con las capacidades de enfriamiento para cada caso. Se simula un flujo de calor de $-146,3 \mathrm{~W}$ para el caso A, $-151,4 \mathrm{~W}$ para el caso B y $-193,7 \mathrm{~W}$ para el caso $\mathrm{C}$.

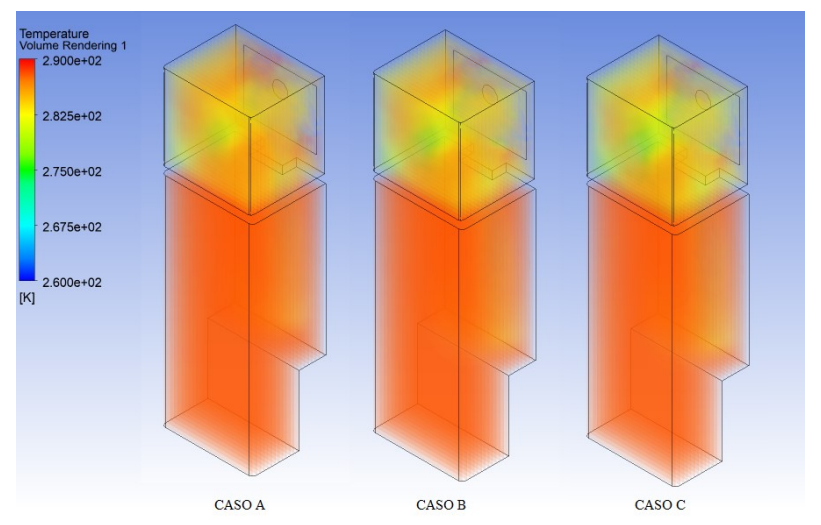

Figura 14. Volume rendering de temperatura en el volumen de control para los casos A, B y C

Las temperaturas alcanzadas en el congelador y el refrigerador no tienen una gran variación en los tres casos de estudio. En la Figura 15 se esquematizan los resultados de las temperaturas en varios puntos del congelador y en la Figura 16 los resultados para partículas en el enfriador.

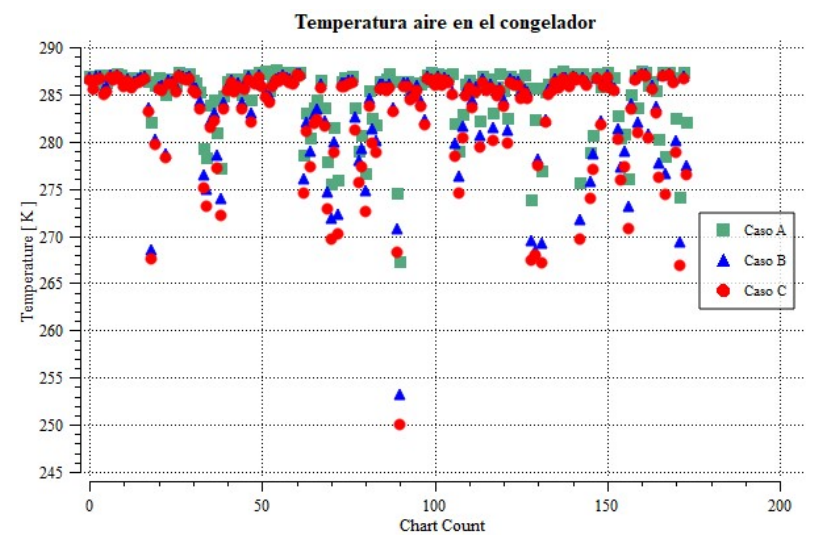

Figura 15. Comparación de temperaturas en el congelador para los casos A, B y C

La temperatura en el congelador en el caso A alcanza un valor promedio de $-6{ }^{\circ} \mathrm{C}$, con picos de hasta $-8{ }^{\circ} \mathrm{C}$; en el caso $\mathrm{B}$ se alcanza un valor promedio de $-8^{\circ} \mathrm{C}$, con picos de hasta $-18{ }^{\circ} \mathrm{C}$; y en el caso $\mathrm{C}$ con rediseño en el sistema se alcanza un valor promedio de $-12{ }^{\circ} \mathrm{C}$, con picos de hasta $-22{ }^{\circ} \mathrm{C}$.

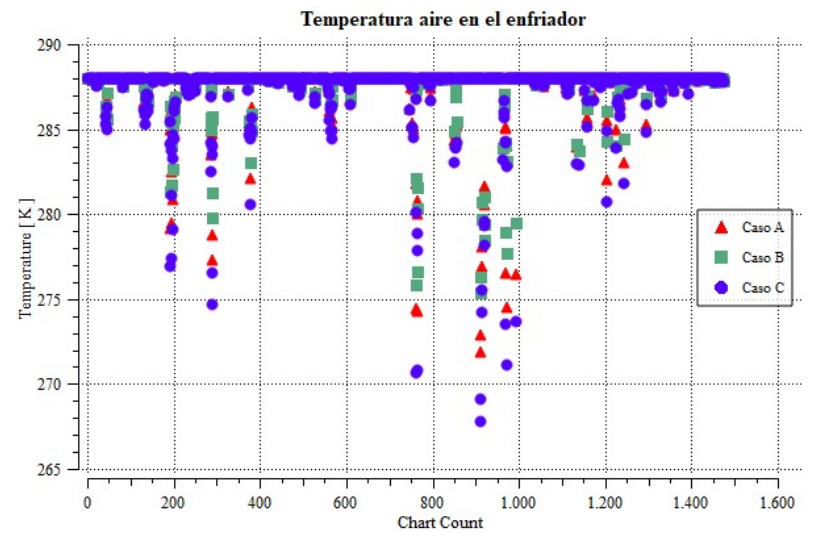

Figura 16. Comparación de temperaturas en el enfriador para los casos A, B y C

La temperatura en el enfriador con el caso A alcanza un valor promedio de $6{ }^{\circ} \mathrm{C}$ con picos de hasta $2{ }^{\circ} \mathrm{C}$; en el caso B se alcanza un valor promedio de $4{ }^{\circ} \mathrm{C}$, con picos de hasta $0{ }^{\circ} \mathrm{C}$ y en el caso $\mathrm{C}$ se alcanza un valor promedio de $2{ }^{\circ} \mathrm{C}$, con picos de hasta $-4{ }^{\circ} \mathrm{C}$. Estos resultados no toman en cuenta pérdidas por entrada de aire al abrir las puertas.

\section{Conclusiones}

Las sustancias de la familia HFO se les considera como la cuarta generación en refrigerantes florados, esto debido a sus propiedades físicas y ambientales y son una excelente opción para diseñar sistemas de refrigeración o aire acondicionado. El HFO-1234ze es una de estas opciones, el cual por sus características es un muy probable remplazo para R134a en sistemas de refrigeración doméstica. Presenta un aporte al calentamiento global 99,9\% más bajo que el R134a y $75 \%$ más bajo que el R600a, los refrigerantes más usados en refrigeración doméstica y por sus características de nula toxicidad y ligeramente inflamable, cumple con los requisitos ambientales y de seguridad necesarios en la actualidad.

Con los resultados obtenidos en las pruebas termodinámicas y de transferencia de calor realizadas con Genetron Properties y ANSYS Fluent, además del análisis matemático, se puede concluir que el refrigerante HFO-1234ze es apto para trabajar en un refrigerador doméstico, mostrando parámetros de refrigeración necesarios sin presentar el costo ambiental de los HFC convencionales y la alta inflamabilidad de los hidrocarburos.

Con las mismas condiciones de funcionamiento en un refrigerador doméstico, el HFO presenta una capacidad de enfriamiento únicamente $8 \%$ más baja que el HFC-134a; con adaptaciones en el proceso de compresión se logra una capacidad de enfriamiento $5 \%$ más baja y rediseñando el sistema para diferentes temperaturas de trabajo se tiene una capacidad 
de enfriamiento $20 \%$ superior; sin embargo, con este rediseño la eficiencia energética disminuye un $2 \%$. El coeficiente de desempeño (COP) del ciclo es de 1,57 con R134a, 1,41 para R1234ze sin ajustes, 1,46 para R1234ze con mejoras al compresor y 1,8 para R1234ze con rediseño.

La temperatura de descarga en el compresor es notablemente inferior con el refrigerante R1234ze en comparación al R134a, siendo en promedio $16{ }^{\circ} \mathrm{C}$ inferior, lo que favorece mucho a la vida útil del compresor y el consumo energético del mismo. También se pudo observar que la temperatura de succión no tiene variaciones importantes entre los dos fluidos y que, en todos los casos, se entrega vapor no húmedo al compresor. Las temperaturas que se logran en el interior del refrigerador tanto en el congelador como en el enfriador son muy similares a las experimentales del refrigerador de referencia con lo cual se espera conseguir una correcta refrigeración al usar el refrigerante R1234ze.

Para realizar adaptaciones físicas a un sistema de refrigeración y trabajar con R134ze, se deben tomar en cuenta los datos sobre la capacidad de enfriamiento, las temperaturas y presiones de trabajo. Se tiene que aprovechar las características termodinámicas del refrigerante en el ciclo, ya que el refrigerante trabaja mejor con temperaturas de evaporación más altas y por sus propiedades lo hace mejor en el proceso de compresión. En futuras investigaciones se recomienda estudiar mezclas de HFO con HFC para remplazar al segundo en totalidad sin tener que remplazar los dispositivos que componen el sistema de refrigeración.

\section{Referencias}

[1] ONU. (2019) Día Internacional de la Preservación de la capa de ozono, 16 de septiembre. [Online]. Available: https://bit.ly/31DBnQf

[2] D. Fahey, P. A. Newman, J. A. Pyle, B. Safari, M. P. Chipperfield, D. Karoly, D. E. Kinnison, M. Ko, M. Santee, and S. J. Doherty, Scientific Assessment of Ozone Depletion: 2018, Global Ozone Research and Monitoring Project-Report No. 58. World Meteorological Organization, 01 2018. [Online]. Available: https://bit.ly/3oil19H

[3] L. Höglund-Isaksson, P. Purohit, M. Amann, I. Bertok, P. Rafaj, W. Schöpp, and J. Borken-Kleefeld, "Cost estimates of the kigali amendment to phase-down hydrofluorocarbons," Environmental Science \& Policy, vol. 75, pp. 138-147, 2017. [Online]. Available: https://doi.org/10.1016/j.envsci.2017.05.006

[4] A. Mota-Babiloni, J. Navarro-Esbrí, A. Barragán, F. Molés, and B. Peris, "Drop-in energy performance evaluation of r1234yf and r1234ze(e) in a vapor compression system as r134a replacements,"
Applied Thermal Engineering, vol. 71, no. 1, pp. 259-265, 2014. [Online]. Available: https: //doi.org/10.1016/j.applthermaleng.2014.06.056

[5] A. Yataganbaba, A. Kilicarslan, and I. Kurtbaş, "Exergy analysis of r1234yf and r1234ze as r134a replacements in a two evaporator vapour compression refrigeration system," International Journal of Refrigeration, vol. 60, pp. 26-37, 2015. [Online]. Available: https://doi.org/10.1016/j.ijrefrig.2015.08.010

[6] M. O. McLinden, A. F. Kazakov, J. Steven Brown, and P. A. Domanski, "A thermodynamic analysis of refrigerants: Possibilities and tradeoffs for Low-GWP refrigerants," International Journal of Refrigeration, vol. 38, pp. 80-92, 2014. [Online]. Available: https://doi.org/10.1016/j.ijrefrig.2013.09.032

[7] D. Sánchez, I. Arauzo, J. Catalán Gil, R. Cabello, R. Doménech, and E. Torrella, "Evaluación energética de una instalación frigorífica empleando refrigerantes de bajo GWP," in CYTEF 2016 - VIII Congreso Ibérico / VI Congreso Iberoamericano de las Ciencias y Técnicas del Frío Coimbra-Portugal, 3-6 mayo, 2016, 052016. [Online]. Available: https://bit.ly/31EdI29

[8] W. C. Whitman and W. M. Jonhson, Tecnología de la refrigeración y aire acondicionado Tomo II. Editorial Paraninfo, 2000. [Online]. Available: https://bit.ly/34u3xiN

[9] N. Jara and C. Isaza-Roldan, "Análisis comparativo de sistemas de refrigeración doméstica utilizando refrigerantes R600a y R134a," Revista I $+T+C$ : Investigación, Tecnología y Ciencia, vol. 1, pp. 7-15, 08 2015. [Online]. Available: https://bit.ly/2TnGIXo

[10] GASSERVEI, "Ficha técnica R-1234ze," GASSERVEI, Tech. Rep., 2020. [Online]. Available: https://bit.ly/3dUDJ2e

[11] Honeywell, "The environmental alternative to traditional refrigerants. solstice ze refrigerant (HFO-1234ze (E))," Honeywell, Tech. Rep., 2018. [Online]. Available: https://bit.ly/3mhCY6d

[12] N. A. Lai, "Equations of state for HFO-1234ze(E) and their application in the study on refrigeration cycle," International Journal of Refrigeration, vol. 43, pp. 194-202, 2014. [Online]. Available: https://doi.org/10.1016/j.ijrefrig.2013.11.011

[13] E. W. Lemmon, I. H. Bell, M. L. Huber, and M. O. McLinden, REFPROP Documentation Release 10.0. National Institute of Standards and Technology (NIST), 2018. [Online]. Available: https://bit.ly/3omeSct 
[14] K. A. Klein and F. L. Alvarado, EES-Engineering Equation Solver. Version 6.648 ND, F-Chart Software, Middleton, 2004.

[15] G. A. Longo, S. Mancin, G. Righetti, and C. Zilio, "R1234yf and R1234ze(e) as environmentally friendly replacements of R134a: Assessing flow boiling on an experimental basis," International Journal of Refrigeration, vol. 108, pp. 336-346, 2019. [Online]. Available: https://doi.org/10.1016/j.ijrefrig.2019.09.008

[16] D. Sánchez, R. Cabello, R. Llopis, I. Arauzo, J. Catalán-Gil, and E. Torrella, "Energy performance evaluation of R1234yf, R1234ze(e), R600a,
R290 and R152a as low-GWP R134a alternatives," International Journal of Refrigeration, vol. 74, pp. 269-282, 2017. [Online]. Available: https://doi.org/10.1016/j.ijrefrig.2016.09.020

[17] ANSYS, ANSYS Fluent Theory Guide. SAS IP, Inc., 2013. [Online]. Available: https://bit.ly/3jk7GtC

[18] H. Benjumea, C. Isaza-Roldan, S. Rio, N. Jara, and J. Ospina, "Simulación del flujo de aire al interior de un refrigerador doméstico nofrost," in VII Congreso Ibérico de Ciencias y Técnicas del Frío, 06 2014. [Online]. Available: https://bit.ly/2TnHwvE 


\title{
IMPACTO DE LOS ALGORITMOS DE SOBREMUESTREO EN LA CLASIFICACIÓN DE SUBTIPOS PRINCIPALES DEL SÍNDROME DE GUILLAIN-BARRÉ
}

\section{IMPACT OF OVERSAMPLING ALGORITHMS IN THE CLASSIFICATION OF GUILLAIN-BARRÉ SYNDROME MAIN SUBTYPES}

\author{
Manuel Torres-Vásquez ${ }^{1,2}$, José Hernández-Torruco ${ }^{1}$, \\ Betania Hernández-Ocaña ${ }^{1}$, Oscar Chávez-Bosquez ${ }^{1 *}$
}

Recibido: 15-05-2020, Revisado: 22-07-2020, Aprobado tras revisión: 25-09-2020

\section{Resumen}

El síndrome de Guillain-Barré es un trastorno neurológico donde el sistema inmune del cuerpo ataca al sistema nervioso periférico. Esta enfermedad es de rápida evolución y es la causa más frecuente de parálisis del cuerpo. Existen cuatro variantes de SGB: polineuropatía desmielinizante inflamatoria aguda, neuropatía axonal motora aguda, neuropatía axonal sensorial aguda y síndrome de Miller-Fisher. Identificar el subtipo de SGB que el paciente contrajo es determinante debido a que el tratamiento es diferente para cada subtipo. El objetivo de este estudio fue determinar cuál algoritmo de sobremuestreo mejora el rendimiento de los clasificadores. Además, determinar si balancear los datos mejoran el rendimiento de los modelos predictivos. Aplicamos tres métodos de sobremuestro (ROS, SMOTE y ADASYN) a la clase minoritaria, utilizamos tres clasificadores (C4.5, SVM y JRip). El rendimiento de los modelos se obtuvo mediante la curva ROC. Los resultados muestran que balancear el dataset mejora el rendimiento de los modelos predictivos. El algoritmo SMOTE fue el mejor método de balanceo en combinación con el clasificador JRip para OVO y el clasificador C4.5 para OVA.

Palabras clave: ADASYN, clasificadores, desbalanceo, ROS, SMOTE, Wilcoxon.

\section{Abstract}

Guillain-Barré Syndrome (GBS) is a neurological disorder where the body's immune system attacks the peripheral nervous system. This disease evolves rapidly and is the most frequent cause of paralysis of the body. There are four variants of GBS: Acute Inflammatory Demyelinating Polyneuropathy, Acute Motor Axonal Neuropathy, Acute Sensory Axial Neuropathy, and Miller-Fisher Syndrome. Identifying the GBS subtype that the patient has is decisive because the treatment is different for each subtype. The objective of this study was to determine which oversampling algorithm improves classifier performance. In addition, to determine whether balancing the data improves the performance of the predictive models. Three oversampling methods (ROS, SMOTE, and ADASYN) were applied to the minority class. Three classifiers (C4.5, SVM and JRip) were used. The performance of the models was obtained using the ROC curve. Results show that balancing the dataset improves the performance of the predictive models. The SMOTE Algorithm was the best balancing method, in combination with the classifier JRip for OVO and the classifier C4.5 for OVA.

Keywords: ADASYN, Classifiers, Unbalance, ROS, SMOTE, Wilcoxon.

\footnotetext{
$\overline{1, *}$ División Académica de Ciencias y Tecnologías de la Información, Universidad Juárez Autónoma de Tabasco, Cunduacán, Tabasco, México. Autor para correspondencia ${ }^{-}$oscar.chavez@ujat.mx.

(D) https://orcid.org/0000-0001-8475-0914 (D) https://orcid.org/0000-0003-3146-9349

(D) https://orcid.org/0000-0001-5700-7615 (D) https://orcid.org/0000-0002-0324-9886

${ }^{2}$ Tecnológico Nacional de México campus Centla, División Sistemas Computacionales, Frontera, Centla, Tabasco, México.

Forma sugerida de citación: Torres-Vásquez, M.; Hernández-Torruco, J.; Hernández-Ocaña, B. y Chávez-Bosquez, O. (2021). «Impacto de los algoritmos de sobremuestreo en la clasificación de subtipos principales del síndrome de Guillain-Barré». InGEnIUs. N. ${ }^{\circ}$ 25, (enero-junio). pp. 20-31. DOI: https://doi.org/10.17163/ings.n25.2021.02.
} 


\section{Introducción}

El síndrome de Guillain-Barré (SGB) se define como una polirradiculoneuropatía autoinmune y es la causa más frecuente de parálisis generalizada aguda [1]. El SGB ocurre cuando el sistema inmunitario ataca parte del sistema nervioso periférico. Esta enfermedad es de rápida evolución y es caracterizada con debilidad en las piernas avanzando hacia los brazos, «parálisis ascendente». Los primeros síntomas son debilidad muscular y hormigueo en las extremidades. Los casos graves requieren de ventilación mecánica. Se desconoce la causa, sin embargo, dos tercios de los casos anteceden a una infección respiratoria o gastroenteritis aguda. Recientemente se ha asociado al virus del Zika. El SGB afecta entre 0.4 y 2.4 casos por 100,000 habitantes/año. Se presenta a cualquier edad, sin embargo, suele tener mayor frecuencia en personas de entre 50 y 80 años. Es ligeramente más frecuente en el hombre que en la mujer. Tiene una tasa de mortalidad de entre 2 y $8 \%$. La mayoría de las personas eventualmente se recuperan completamente cuando la afección es ligera o moderada, en otros casos pueden quedar con daños en el sistema nervioso por mucho tiempo o incluso en forma permanente [2]. Los estudios electrofisiológicos y de conducción nerviosa determinan las pruebas para el diagnóstico de SGB. Existen cuatro subtipos principales de SGB:

- Polineuropatía desmielinizante inflamatoria aguda (AIDP).

- Neuropatía axonal motora aguda (AMAN).

- Neuropatía axonal sensorial aguda (AMSAN).

- Síndrome de Miller-Fisher (MF).

La recuperación del paciente depende en gran medida de la pronta identificación del subtipo de SGB. Cada subtipo debe tratarse de manera diferente, el tratamiento y los costos varían según el subtipo desarrollado por el paciente. En los casos graves que generan inmovilidad transitoria o permanente, las terapias de rehabilitación suelen ser tardadas y costosas generando repercusiones psicológicas y económicas al enfermo y a los familiares.

El aprendizaje automático o Machine Learning es una rama de la Inteligencia Artificial que utiliza diversas técnicas matemáticas, estadísticas, y de optimización, con el objetivo de desarrollar herramientas de análisis de información para que las computadoras «aprendan» a través de ejemplos [3]. En la actualidad disciplinas como finanzas, petróleo, mercadeo, ventas y salud utilizan el aprendizaje automático como herramienta tecnológica para hacer predicciones. Específicamente en el área de la salud se desarrollan cada vez más modelos para el diagnóstico de enfermedades como son el cáncer [4], [5], diabetes [6], [7], Parkinson [8] y Alzheimer [9] dando excelentes resultados.

Los algoritmos de clasificación son los encargados de analizar los datos proporcionados y determinan los pacientes que están sanos y los que se encuentran enfermos. Sin embargo, uno de los problemas más comunes en el diagnóstico médico es la desproporcionalidad de casos. En la vida real existen más casos de pacientes sanos que de pacientes enfermos. Por ejemplo, si queremos diagnosticar pacientes con diabetes, encontramos que un mayor número de gente está sana y un menor número padece diabetes. A esta desproporción de datos se le llama desbalanceo de datos. Existen dos tipos de desbalanceo: desbalanceo binario y desbalanceo multiclase. El desbalanceo binario se presenta cuando en un conjunto de datos formado por dos clases, una tiene un mayor número de datos (clase mayoritaria) respecto a la otra (clase minoritaria). Por otro lado, el desbalanceo multiclase se presenta cuando un conjunto de datos lo forman más de dos clases y su distribución de datos es desigual para cada una de las clases [10].

El desbalanceo de datos puede afectar el resultado de los clasificadores ya que tienden a sesgar sus resultados hacia la clase mayoritaria (pacientes sanos). Los algoritmos de clasificación estándar están construidos para datos balanceados, es decir, el mismo número de casos sanos y casos enfermos. Por ejemplo, para el caso de los pacientes con diabetes, el clasificador ignorará los pacientes con diabetes y solo tomará en cuenta los pacientes sanos. El problema es que queremos determinar los pacientes enfermos y no los pacientes sanos. Es por eso que es necesario utilizar técnicas que ayuden a balancear los datos.

En la literatura especializada existen tres técnicas más comunes para resolver el problema del desbalanceo de datos [11].

- Nivel de datos. Esta técnica agrega o elimina datos a la clase hasta equilibrar el dataset. Esta técnica también se le conoce como de muestreo y está dividida en tres grupos:

- Sobremuestreo: consiste en agregar datos a la clase minoritaria hasta lograr el equilibrio con la clase mayoritaria.

- Submuestreo: consiste en eliminar datos de la clase mayoritaria hasta alcanzar el equilibrio con la clase minoritaria.

- Híbridos: esta técnica combina el sobremuestreo y el submuestreo al mismo tiempo para lograr un mejor equilibrio entre clases.

- Nivel algoritmo. Adaptan o crean algoritmos de clasificación para reforzar la predicción de la clase.

- Costo sensitivo. Consideran los costos asociados con clasificación errónea de las muestras. Uti- 
liza diferentes matrices de costo que describen los costos de clasificar erróneamente cualquier ejemplo de datos en particular.

La técnica a nivel de datos es una de las más utilizadas ya que es independiente del clasificador que se utiliza; además, los datos son tratados antes de ser utilizados por el clasificador. La técnica de sobremuestreo es la más empleada ya que agrega datos a la clase minoritaria. Existen diferentes técnicas de sobremuestreo que generan datos dando buenos resultados respecto al submuestreo que puede llegar a eliminar datos de importancia y afectar el resultado del clasificador [12].

Por otro lado, además del desbalanceo de los datos, la distribución de las instancias afecta el resultado de los clasificadores [13]. Existen técnicas que agregan datos sintéticos a la clase minoritaria y las colocan en lugares estratégicos para solventar el problema del desbalanceo y la posición de las instancias.

El objetivo de este estudio fue doble. El primero fue identificar cuál de los tres algoritmos de sobremuestreo utilizados para balancear el dataset de SGB original mejora el resultado de los algoritmos de clasificación. El segundo objetivo fue establecer si balancear los datos mejora el rendimiento de los modelos predictivos creados con datos balanceados, respecto a los modelos creados con datos desbalanceados. Para esto, utilizamos la prueba estadística Wilcoxon para conocer si existe diferencia estadísticamente significativa entre dichos modelos. Actualmente, no existe en la literatura especializada estudios para identificar los subtipos principales de SGB utilizando algoritmos de aprendizaje automático. En estudios previos [14], [15], se crearon modelos predictivos utilizando el dataset original desbalanceado. En este estudio experimental, balanceamos los subsets de entrenamiento utilizando tres técnicas de sobremuestreo (ROS, SMOTE y ADASYN). Los resultados demuestran que balancear los datos mejoran el rendimiento de los modelos predictivos. En algunos casos se logró un rendimiento de $90 \%$.

Para este estudio, primero utilizamos dos técnicas de binarización (OVO y OVA) para crear diez subsets binarios. Después dividimos los subsets en sets de entrenamiento con un $66 \%$ de los datos y sets de prueba con un $33 \%$ de los datos. Una vez obtenidos los datos de entrenamiento, se les aplicaron tres métodos de balanceo (ROS, SMOTE y ADASYN), para sobremuestrear la clase minoritaria y equilibrarla con la clase mayoritaria. Una vez balanceado los datos se aplicaron tres algoritmos de clasificación con diferentes enfoques: C4.5 (árbol de decisión), SVM (Support Vector Machine), JRip (Ripper). El rendimiento de los modelos predictivos se determinó utilizando el área bajo la curva (AUC) de la curva ROC. Los resultados de los modelos predictivos son el promedio del AUC de 60 ejecuciones. Finalizamos aplicando la prueba Wilcoxon a los modelos creados con datos balanceados que superaron el rendimiento de los modelos creados con los datos desbalanceados, para conocer si existe diferencia estadísticamente significativa entre dichos modelos.

\section{Materiales y métodos}

\subsection{Dataset}

El dataset utilizado en este estudio es una recopilación de 129 pacientes diagnosticados con SGB. A cada uno de los pacientes se les identificó con alguno de los cuatro subtipos principales de SGB. En la Tabla 1 se muestran las características principales del dataset.

Tabla 1. Características del dataset

\begin{tabular}{lr}
\hline Característica & Valor \\
\hline Número de clases & 4 \\
Número de instancias & 129 \\
Número de atributos & 16 \\
Instancias Clase 1 (AIDP) & 20 \\
Instancias Clase 2 (AMAN) & 37 \\
Instancias Clase 3 (AMSAN) & 59 \\
Instancias Clase 4 (MF) & 13 \\
\hline
\end{tabular}

Esta información se obtuvo a través del Instituto Nacional de Neurología y Neurocirugía de la Ciudad de México. El dataset original consta de 356 variables. En un artículo anterior se identificaron 16 variables como las más relevantes [16]. Las primeras 4 variables son de tipo clínico, las siguientes 14 variables pertenecen a la prueba de conducción nerviosa. A continuación se muestran las variables utilizadas en los experimentos:

v22: Simetría (en debilidad)

v29: Afectación de los músculos extraoculares

v30: Ptosis

v31: Implicación cerebelosa

v63: Amplitud del nervio motor mediano izquierdo

v106: Área bajo la curva del nervio motor cubital izquierdo v120: Área bajo la curva del nervio motor cubital derecho v130: Amplitud del nervio motor tibial izquierdo

v141: Amplitud del nervio motor tibial derecho

v161: Área bajo la curva del nervio motor peroneo derecho v172: Amplitud del nervio sensorial mediano izquierdo

v177: Amplitud del nervio sensorial mediano derecho

v178: Área bajo la curva del nervio sensorial mediano derecho

v186: Latencia del nervio sensorial cubital derecho

v187: Amplitud del nervio sensorial cubital derecho

v198: Área bajo la curva del nervio sensorial sural derecho 


\subsection{Algoritmos de aprendizaje automático}

\subsubsection{Algoritmos de sobremuestro}

Los algoritmos de sobremuestreo son una técnica a nivel de datos que agregan datos a la clase minoritaria con el objetivo de equilibrar el conjunto desbalanceado de datos. Existen diversos algoritmos para sobremuestrear las clases. Para este estudio utilizamos tres técnicas que generan instancias con diferentes enfoques:

1. El algoritmo de sobremuestreo aleatorio ROS (Random Oversampling), obtiene una muestra al azar de instancias de la clase minoritaria y realiza una copia de ellas. Las instancias duplicadas se colocan en forma aleatoria dentro del dataset. ROS es un método no heurístico que tiene como objetivo balancear la clase minoritaria con la mayoritaria [17].

2. El algoritmo de sobremuestro sintético SMOTE (Synthetic Minority Oversampling Technique) sobremuestrea la clase minoritaria generando instancias sintéticas con el objetivo de equilibrarla con la mayoritaria [18]. Las nuevas instancias sintéticas se generan a través de la interpolación entre varias instancias de clases minoritarias basándose en la regla del vecino más cercano. SMOTE realiza este procedimiento en el «espacio de características». El procedimiento para generar los datos sintéticos es el siguiente: (a) Se determina el porcentaje de sobremuestreo que se necesita generar. (b) Para generar los objetos sintéticos realiza el siguiente proceso: (b1) Selecciona una instancia de clase minoritaria al azar. (b2) Elige aleatoriamente sus k-vecinos más cercanos de acuerdo con la distancia euclidiana. (b3) Se toma la diferencia entre el vector de características y cada uno de los vecinos seleccionados. (b4) Esta diferencia se multiplica por un número aleatorio 0 y 1. (b5) Suma este último valor al valor original de la muestra. (b6) Devuelve la muestra sintética. (c) La nueva muestra sintética se colocará entre la instancia seleccionada originalmente y cada uno de los k-vecinos más cercanos.

La diferencia principal entre SMOTE y ROS es que ROS duplica datos de la clase minoritaria y las agrega en forma aleatoria. SMOTE genera datos sintéticos y los ubica en un vecindario de la clase minoritaria.

3. El algoritmo de enfoque de muestreo sintético adaptativo llamado ADASYN, (Adaptive Synthetic Sampling Approach for Imbalanced Learning), es una extensión de SMOTE. ADASYN tiene dos objetivos: el primero es crear instancias sintéticas a través de la interpolación lineal, entre las instancias de la clase minoritaria para reducir su desequilibrio con la clase mayoritaria del dataset. El segundo objetivo que hace diferente a ADASYN respecto a SMOTE es que los datos generados cambian adaptivamente el límite de decisión agregando datos en la zona de la clase minoritaria difícil de aprender en comparación de los datos de la clase minoritaria fáciles de aprender, esto a través de una distribución de densidad. ADASYN busca darle mayor peso a los datos de la clase minoritaria que son difíciles de aprender [19].

\subsubsection{Algoritmos de clasificación}

Se utilizan tres algoritmos de clasificación que determinan sus resultados a través de diferentes enfoques. El objetivo es contrastar los resultados de cada uno de ellos:

1. Árbol de decisión (C4.5). Es un algoritmo de aprendizaje supervisado en el que cada nodo de rama representa una elección entre varias opciones y cada nodo de hoja representa una decisión. La técnica de clasificación la realiza mediante criterios de división, con una estructura de árbol invertido, similar a un diagrama de flujo. Maneja características continuas y discretas. Tiene alta precisión, estabilidad, es rápido, fácil de interpretar y robusto al ruido. C4.5 basa sus resultados en forma jerárquica y de aprendizaje inductivo, es decir, en el descubrimiento de patrones a partir de ejemplos [20].

2. Máquina de vector soporte (SVM). Es un algoritmo de aprendizaje supervisado que se emplea para clasificación binaria. Pertenece a la familia de clasificadores lineales, esto es, mediante una función matemática los datos originales se redimensionan para buscar una separabilidad lineal de los mismos. SVM se basa en el concepto de construir un hiperplano óptimo, es decir, crea una recta que separa a las clases. El objetivo es encontrar el mejor hiperplano que divida mejor el conjunto de datos y maximice el margen entre las clases [21].

3. Ripper (JRip). Es uno de los algoritmos más populares para problemas de clasificación, con un enfoque basado en reglas. Las clases se examinan en tamaño creciente y se genera un conjunto inicial de reglas para la clase usando el error incremental reducido JRip (RIPPER). Procede tratando todos los ejemplos de un juicio particular en los datos de entrenamiento como una clase, y encontrando un conjunto de reglas que cubrir a todos los miembros de esa clase. Posteriormente, 
pasa a la siguiente clase y hace lo mismo, repitiendo esto hasta que se hayan cubierto todas las clases [22].

\subsection{Medida de rendimiento}

Se evalúa el rendimiento de los algoritmos de clasificación utilizando el gráfico de características operativas del receptor o curva ROC (Receiver Operating Characteristic) y el área bajo la curva AUC (area under the curve). La curva ROC mide qué tan bien se clasifican las predicciones, así como la calidad de las predicciones del modelo [23]. La curva ROC se define como la sensibilidad, que es la tasa de verdaderos positivos que se muestra en la Ecuación 1. La 1-especificidad es la tasa de falsos positivos, se muestra en la Ecuación 2. Para este experimento, nos sirve para identificar entre uno de los subtipos de SGB.

$$
\begin{gathered}
\text { sensibilidad }=\frac{V P}{V P+F N} \\
1-\text { especificidad }=\frac{F P}{V N+F P}
\end{gathered}
$$

El área bajo la curva AUC permite identificar una clase. Por ejemplo, reconocer si un paciente padece cierta enfermedad o está sano. En esta medida de rendimiento, los valores $\geq .900$ se consideran modelos excelentes. Los valores $\geq .700$ significa que son buenos modelos. Sin embargo, los valores con $\leq .500$ están considerados malos modelos.

\subsection{Técnicas de binarización}

En problemas de clasificación es común encontrar datasets que están formados por más de dos clases, a esto se le llama dataset multiclase. Algunos algoritmos de clasificación solo pueden ser capaces de discriminar entre dos clases. Es por eso por lo que es común convertir un problema multiclase en subproblemas binarios. En la literatura encontramos dos técnicas de binarización utilizadas: uno contra uno (OVO) y uno contra todos (OVA) por sus siglas en inglés [24].

La técnica OVO divide un problema de $n$ clases en $n(n-1) / 2$ subproblemas binarios, formando todos los posibles pares de clases. La técnica OVA toma una clase como clase minoritaria, las demás clases son combinadas para formar la clase mayoritaria. Este procedimiento se realiza $n$ veces según el número de clases que forman el dataset. Se utilizan las técnicas de binarización OVO y OVA para discriminar una clase de las otras. En problemas de diagnóstico médico sirve para identificar un paciente enfermo de otros pacientes sanos. En las Figuras 1 y 2 se muestran los 4 subsets obtenidos con la técnica OVA y los 6 subsets obtenidos con 1-especificidad OVO al dataset original SGB.

\subsection{Validación}

Se valida el modelo para cada clasificación utilizando la evaluación train-test. El dataset se divide en dos subconjuntos de datos. El primero son los datos de entrenamiento, estos se usaron para construir el modelo. El segundo son llamados datos de prueba, estos se mantienen aparte y a través de ellos se evaluó el modelo. Se emplean $\frac{2}{3}$ del conjunto de datos para el entrenamiento y $\frac{1}{3}$ del conjunto de datos para las pruebas del modelo.

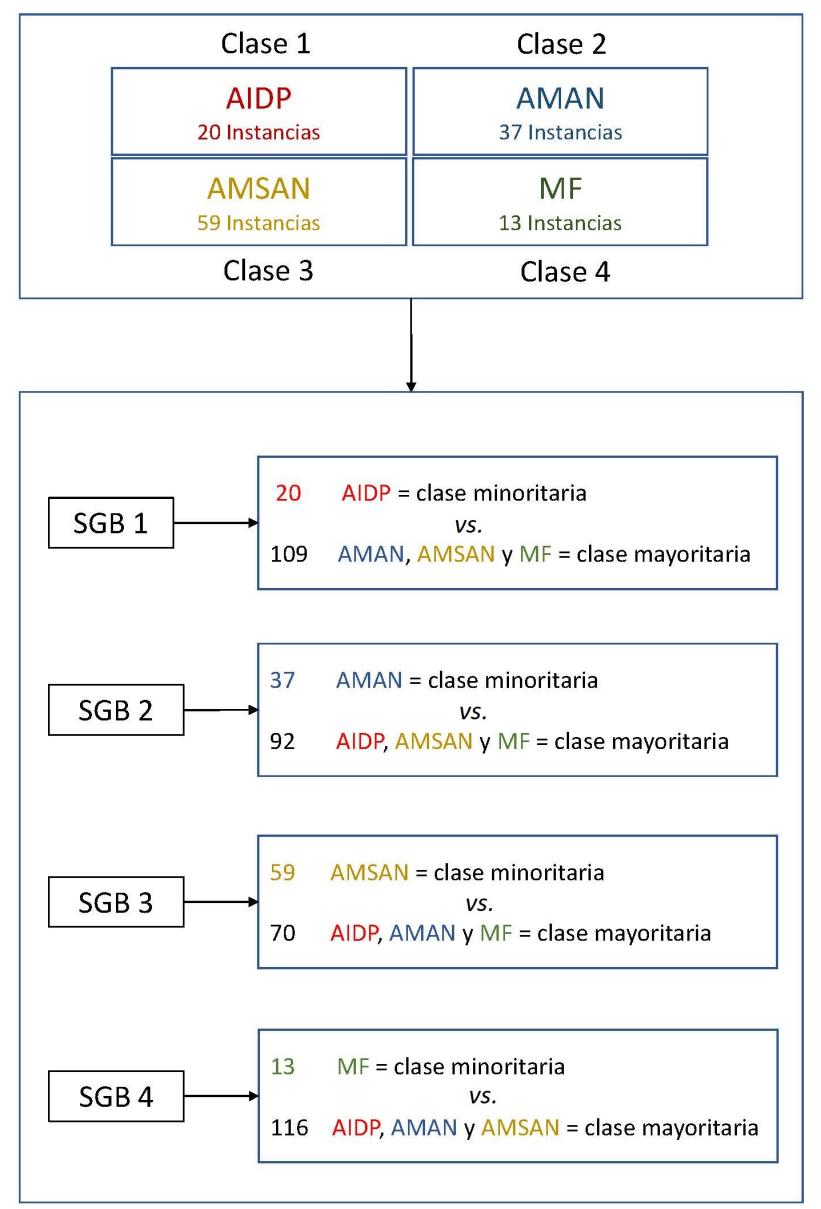

Figura 1. Binarización uno contra todos (OVA)

\section{Procedimiento experimental}

Como primer paso, se toma el dataset original desbalanceado multiclase y se lo convierte en dos subproblemas binarios, utilizando dos técnicas diferentes de binarización (OVO y OVA). La diferencia entre ellas, es que la técnica OVO crea todas las posibles combinaciones que se pueden formar con las $n$ clases que forman un dataset. Por otro lado, la técnica OVA toma una clase para convertirla en clase minoritaria y las clases restantes son combinadas para formar la clase mayoritaria. OVA crea subconjuntos dependiendo del 
Torres-Vásquez et al. / Impacto de los algoritmos de sobremuestreo en la clasificación de subtipos principales del sindrome de Guillain-Barré

total de clases que forman el dataset original. El objetivo de crear subsets binarios es que los métodos de balanceo utilizados en este estudio solo identifican dos clases, la clase minoritaria que se sobremuestrea hasta equilibrarla con la mayoritaria.

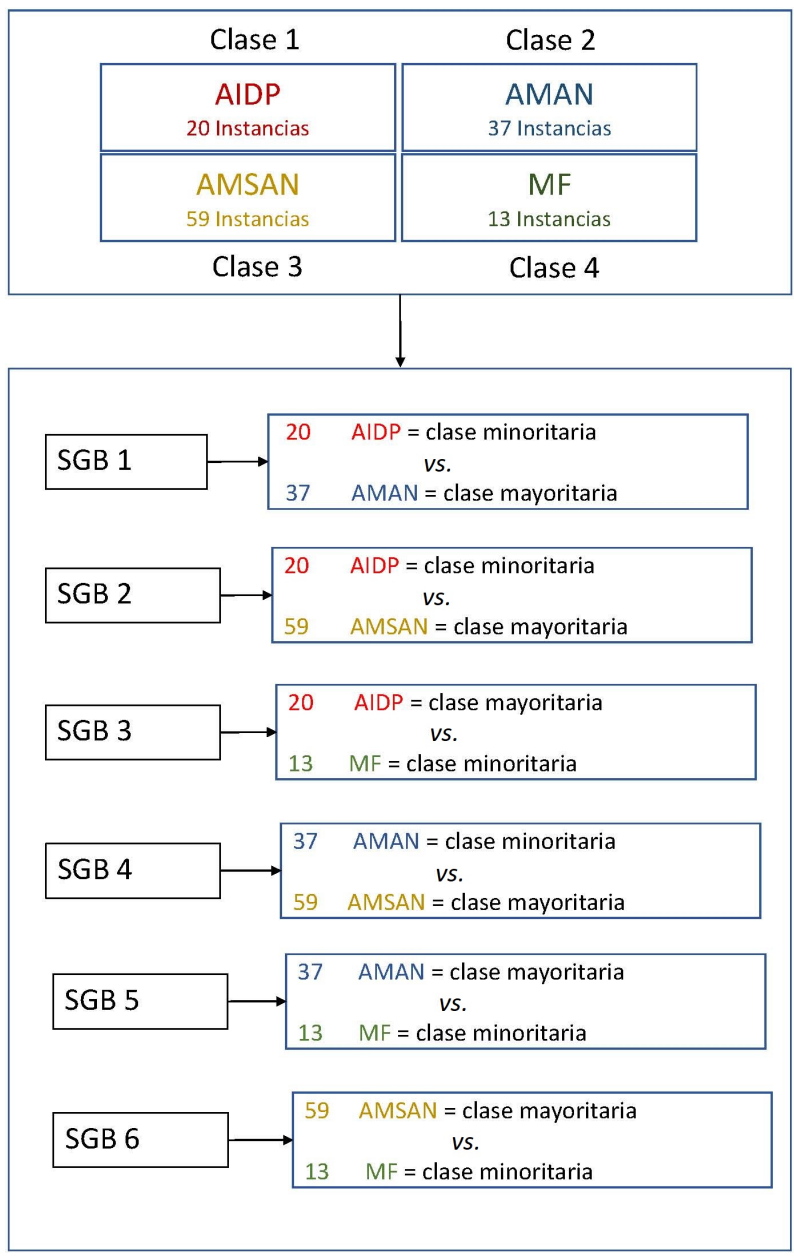

Figura 2. Binarización uno contra uno (OVO)

Tabla 2. Subsets obtenidos con la técnica OVA

\begin{tabular}{ccc}
\hline Subset & $\begin{array}{c}\text { Clase } \\
\text { minoritaria }\end{array}$ & $\begin{array}{c}\text { Clase } \\
\text { mayoritaria }\end{array}$ \\
\hline SGB1 & 20 & 109 \\
SGB2 & 37 & 92 \\
SGB3 & 59 & 70 \\
SGB4 & 13 & 116 \\
\hline
\end{tabular}

Aplicando las dos técnicas de binarización se obtiene un total de 10 datasets binarios. En la Tabla 2 se muestran los 4 dataset creados con la técnica OVA y en la Tabla 3 se muestran los 6 dataset binarios creados con la técnica OVO. En la primera columna se muestran los subsets obtenidos con la técnica de binarización. En la segunda columna se observa el número de instancias que forman la clase minoritaria.
La tercera columna muestra el número de instancias que integran la clase mayoritaria. Podemos observar que la técnica OVA tiene un mayor desbalanceo de datos entre la clase minoritaria y la clase mayoritaria respecto a la técnica OVO.

Tabla 3. Subsets obtenidos con la técnica OVO

\begin{tabular}{lcc}
\hline Subset & $\begin{array}{c}\text { Clase } \\
\text { minoritaria }\end{array}$ & $\begin{array}{c}\text { Clase } \\
\text { mayoritaria }\end{array}$ \\
\hline SGB1 & 20 & 37 \\
SGB2 & 20 & 59 \\
SGB3 & 13 & 20 \\
SGB4 & 37 & 59 \\
SGB5 & 13 & 37 \\
SGB6 & 13 & 59 \\
\hline
\end{tabular}

Como segundo paso, se divide cada uno de los 10 subsets se fraccionan para el entrenamiento los datos en $2 / 3$, y el $1 / 3$ de estos se utilizan para la prueba $\mathrm{A}$ continuación, se aplicaron los tres algoritmos de sobremuestreo (ROS, SMOTE y ADASYN) a la clase minoritaria que pertenecen a los datos de entrenamiento, hasta equilibrarla con la clase mayoritaria. Los datos de prueba se utilizaron para medir el rendimiento de los modelos obtenidos.

Tabla 4. Resultados de los subsets balanceados aplicando los métodos de sobremuestreo a la clase minoritaria para OVA

\begin{tabular}{lcccc}
\hline Subset & Datos A & Datos B & Clase a & Clase b \\
\hline SGB1 & 14 & 59 & 73 & 73 \\
SGB2 & 25 & 37 & 61 & 62 \\
SGB3 & 40 & 7 & 47 & 47 \\
SGB4 & 9 & 69 & 78 & 78 \\
\hline
\end{tabular}

Datos A: Datos de entrenamiento desbalanceados.

Datos B: Datos generados con SMOTE, ROS y ADASYN.

Clase a: Clase minoritaria balanceada.

Clase b: Clase mayoritaria original.

En la Tabla 4 se muestran los 4 subsets balanceados para la técnica OVA. En la Tabla 5 se muestran los 6 subsets balanceados para la técnica OVO. En la primera columna se muestran los subsets de la técnica de binarización. En la segunda columna se muestra la clase minoritaria con el número de instancias que la integran. En la tercera columna se observa el número de instancias que fueron generadas por cada algoritmo de sobremuestreo. Las columnas 4 y 5 muestran la clase minoritaria y la clase mayoritaria balanceadas respectivamente.

El siguiente paso fue obtener los modelos predictivos aplicando tres algoritmos de clasificación (C4.5, SVM y JRip) a los 10 subsets balanceados. Se realizan 60 ejecuciones independientes calculando el área bajo la curva (AUC) para los 10 subsets. Los modelos predictivos son el resultado del promedio del área bajo la curva de las 60 ejecuciones. Por otro lado, se ejecuta 
el mismo procedimiento utilizando los subsets desbalanceados para obtener modelos predictivos con datos desbalanceados.

Tabla 5. Resultados de los subsets balanceados aplicando los métodos de sobremuestreo a la clase minoritaria para OVO

\begin{tabular}{lcccc}
\hline Subset & Datos A & Datos B & Clase a & Clase b \\
\hline SGB1 & 14 & 9 & 23 & 23 \\
SGB2 & 14 & 26 & 40 & 40 \\
SGB3 & 9 & 5 & 14 & 14 \\
SGB4 & 25 & 15 & 40 & 40 \\
SGB5 & 9 & 16 & 25 & 25 \\
SGB6 & 9 & 31 & 40 & 40 \\
\hline
\end{tabular}

Datos A: Datos de entrenamiento desbalanceados

Datos B: Datos generados con SMOTE, ROS y ADASYN

Clase a: Clase minoritaria balanceada

Clase b: Clase mayoritaria original

El último paso fue comparar el rendimiento de los modelos obtenidos con datos balanceados contra los obtenidos con los datos desbalanceados. Se utiliza la prueba estadística de Wilcoxon para conocer si existe una diferencia estadísticamente significativa entre los modelos, siempre y cuando los modelos balanceados hayan superado a los desbalanceados. Se utiliza un valor de significación de 0.05 .

Los experimentos se realizaron en el software R, diseñado para el análisis estadístico. Se utilizó el entorno de desarrollo integrado RStudio versión 1.2.1335. Los paquetes que se utilizan para balancear los datos fueron: el paquete unbalanced para el algoritmo ROS [25], el paquete DMwR para el algoritmo SMOTE [26] y el paquete UBL para el algoritmo ADASYN [27]. Para los algoritmos de clasificación C4.5 y JRip utilizamos el paquete RWeka 0.4-39 [28]. Para el clasificador SVM usamos el paquete e1071 1.7-0 [29].

El clasificador SVM lineal se optimizó a través de la función tune, asignando los valores; 0.001, 0.01, $0.1,1,10,50,80,100$ para el parámetro $C$. Los clasificadores JRip y C45 no requieren optimización de hiperparámetros.

\section{Resultados y discusión}

En las Tablas 6 y 9 se muestran los resultados de los modelos predictivos obtenidos, aplicando tres métodos de balanceo (ROS, SMOTE y ADASYN). Se sobremuestrean seis subsets desbalanceados obtenidos con la técnica de binarización OVO y cuatro subsets obtenidos con la técnica de binarización OVA. Cada valor, es el promedio de los resultados obtenidos a través de 60 ejecuciones. Se aplican los clasificadores C4.5, SVM y JRip una vez balanceado el conjunto de entrenamiento. Los modelos fueron evaluados utilizando la métrica ROC. Se ejecutó la prueba estadística Wilcoxon a los modelos balanceados contra los desbalanceados, cuando el rendimiento de los modelos balanceados superó el rendimiento de los desbalanceados con el objetivo de conocer si el rendimiento del primero obtenía una diferencia estadísticamente significativa.

La estructura de las tablas es la siguiente: la primera columna muestra los subsets obtenidos mediante las técnicas de binarización OVO y OVA, los subtipos de SGB que lo forman, así como la cantidad de instancias para cada subtipo. La segunda columna muestra los tres clasificadores utilizados para obtener los modelos predictivos en cada subset. La tercera columna muestra los resultados de los modelos predictivos utilizando datos desbalanceados. Las columnas 4, 5 y 6 muestran los modelos obtenidos utilizando datos balanceados aplicando tres técnicas de sobremuestreo (ROS, SMOTE y ADASYN). También se observa que, los valores en negrita son los modelos predictivos que, además de superar a los modelos desbalanceados, obtuvieron una diferencia estadísticamente significativa.

En la Tabla 6 se muestran los resultados de los 72 modelos predictivos obtenidos utilizando la técnica de binarización OVO. De estos modelos, 18 fueron creados con datos desbalanceados y 54 se obtuvieron utilizando datos balanceados aplicando tres métodos de sobremuestreo. Se encontraron que 32 modelos balanceados no pudieron superar el rendimiento de los modelos desbalanceados. Otros 15 modelos balanceados superaron el rendimiento de los datos desbalanceados, sin embargo, no se encontró diferencia estadísticamente significativa. Por otro lado, 7 modelos balanceados superaron a los modelos desbalanceados y, además tuvieron una diferencia estadísticamente significativa.

Con el subset SGB6 se encontraron los mejores resultados, al obtener 3 modelos con diferencia estadísticamente significativa. Por otro lado, en los subsets SGB2 y SGB4 tuvieron 2 modelos con diferencia estadísticamente significativa cada uno. Los subsets SGB1, SGB3 y SGB5 obtuvieron el peor rendimiento respecto a los modelos desbalanceados ya que en ninguno de los modelos se encontró diferencia estadísticamente significativa.

Respecto a los métodos de balanceo, en la Tabla 7 se muestran los resultados del ranking obtenido por cada método. Estos resultados se obtuvieron al asignar una posición a cada método dependiendo de su rendimiento con cada subset. Por cada fila, se asigna un valor a cada método de sobremuestreo. En la primera fila, a SMOTE se le asigna el valor 1 ya que obtuvo el mejor rendimiento. A ROS se le asigna el valor 2 ya que obtuvo el siguiente rendimiento y finalmente a ADASYN se le asigna el valor 3 ya que fue el método con el peor rendimiento. Esta operación se realiza para cada fila. Seguidamente, se suman todos los valores por cada método y se dividen por el número de filas para obtener el promedio. Por ejemplo, SMOTE obtuvo 5 veces el primer lugar, 6 veces el segundo lugar, 5 veces el tercer lugar y 2 veces el cuarto lugar. La suma de 
Torres-Vásquez et al. / Impacto de los algoritmos de sobremuestreo en la clasificación de subtipos principales del sindrome de Guillain-Barré

Tabla 6. Tabla de resultados de los modelos predictivos aplicando ROS, SMOTE y ADASYN para sobremuestrear la clase minoritaria

\begin{tabular}{lccccc}
\hline Subset & Clasificador & $\begin{array}{c}\text { Datos } \\
\text { desbalanceados }\end{array}$ & $\begin{array}{c}\text { Balanceo } \\
\text { aplicando } \\
\text { ROS }\end{array}$ & $\begin{array}{c}\text { Balanceo } \\
\text { aplicando } \\
\text { SMOTE }\end{array}$ & $\begin{array}{c}\text { Balanceo } \\
\text { aplicando } \\
\text { ADASYN }\end{array}$ \\
\hline SGB1 & C4.5 & 0.9604 & 0.9514 & 0.9576 & 0.9292 \\
AIDP-AMAN & SVM & 0.9576 & 0.9465 & 0.9618 & 0.9486 \\
$20-37$ & JRip & 0.9563 & 0.9507 & 0.9403 & 0.9396 \\
\hline SGB2 & C4.5 & 0.8585 & 0.8160 & 0.8551 & 0.8529 \\
AIDP-AMSAN & SVM & 0.8472 & 0.8306 & 0.8333 & 0.8484 \\
20-59 & JRip & 0.8260 & 0.8178 & $\mathbf{0 . 8 5 4 9 *}$ & $\mathbf{0 . 8 5 4 5 *}$ \\
\hline SGB3 & C4.5 & 0.8132 & 0.8111 & 0.7965 & 0.7854 \\
AIDP-MF & SVM & 0.6556 & 0.6340 & 0.6535 & 0.6792 \\
20-13 & JRip & 0.8556 & 0.8493 & 0.7382 & 0.8396 \\
\hline SGB4 & C4.5 & 0.9258 & 0.9093 & 0.9093 & 0.8897 \\
AMAN-AMSAN & SVM & 0.8760 & 0.8692 & 0.8827 & 0.8845 \\
37-59 & JRip & 0.8782 & $\mathbf{0 . 9 0 5 9 *}$ & $\mathbf{0 . 9 0 6 5 *}$ & 0.8877 \\
\hline SGB5 & C4.5 & 0.8736 & 0.8826 & 0.8868 & 0.8486 \\
AMAN-MF & SVM & 0.8806 & 0.8729 & 0.8847 & 0.8910 \\
37-13 & JRip & 0.8854 & 0.8958 & 0.8889 & 0.8833 \\
\hline SGB6 & C4.5 & 0.8007 & $\mathbf{0 . 8 4 1 1 *}$ & 0.7839 & 0.8209 \\
AMSAN-MF & SVM & 0.7089 & $\mathbf{0 . 7 6 0 0 *}$ & 0.7534 & $\mathbf{0 . 7 7 4 6 *}$ \\
59-13 & JRip & 0.8580 & 0.8561 & 0.8720 & 0.8264 \\
\hline
\end{tabular}

Los valores son el promedio de 60 ejecuciones de las curvas ROC utilizando OVO.

estos valores es de 40 y se divide por el número de filas de la tabla, para este caso es 18 . El resultado es 2.222 y al ser el promedio más bajo, ocupa el número 1 en el ranking [30].

Para OVO, el algoritmo SMOTE fue el método de balanceo con el mejor rendimiento con una puntuación promedio de 2.2222. Los algoritmos ADASYN y ROS, ocuparon el segundo lugar al obtener la misma puntuación promedio de 2.7222 .

Respecto a los clasificadores, en la Tabla 8 se muestra que el clasificador JRip, obtuvo el mejor rendimiento con una puntuación promedio de 1.6667 . El clasificador C4.5 obtuvo el segundo lugar con una puntuación promedio de 1.8333. Por último, el clasificador SVM obtuvo el peor rendimiento con una puntuación promedio de 2.500 .

Tabla 7. Resultados del ranking por método de balanceo para OVO

\begin{tabular}{lcc}
\hline Método & Ranking & Puntuación promedio \\
\hline SMOTE & 1 & 2.2222 \\
ADASYN & 2 & 2.7222 \\
ROS & 2 & 2.7222 \\
\hline
\end{tabular}

En la Tabla 9 se muestran los resultados de 48 modelos predictivos, obtenidos utilizando la técnica de binarización OVA. De estos, 12 modelos fueron creados con datos desbalanceados y 36 modelos se obtuvieron utilizando datos balanceados aplicando tres métodos de sobremuestreo. Se encontró que 15 modelos balanceados no pudieron superar el rendimiento de los modelos desbalanceados. En 9 modelos balanceados superaron el rendimiento de los datos desbalanceados, sin embargo, no se encontró diferencia estadísticamente significativa. Por otro lado, 12 modelos balanceados superaron a los datos desbalanceados $\mathrm{y}$, además, tuvieron una diferencia estadísticamente significativa.

Tabla 8. Resultados del ranking por clasificador para OVO

\begin{tabular}{lcc}
\hline Clasificador & Ranking & Puntuación promedio \\
\hline JRip & 1 & 1.6667 \\
C4.5 & 2 & 1.8333 \\
SVM & 3 & 2.5000 \\
\hline
\end{tabular}

Con los subsets SGB1 y SGB4 obtuvieron los mejores rendimientos. En el subset SGB1, 8 modelos balanceados mejoraron los modelos desbalanceados, de los cuales, 5 obtuvieron diferencia estadísticamente significativa. En el subset SGB4, 6 modelos balanceados superaron los datos desbalanceados, de estos, 5 modelos obtuvieron diferencia estadísticamente significativa. Con el subset SGB2, 5 modelos balanceados superaron a los modelos desbalanceados, sin embargo, solo 2 
Tabla 9. Tabla de resultados de los modelos predictivos aplicando ROS, SMOTE y ADASYN para sobremuestrear la clase minoritaria

\begin{tabular}{|c|c|c|c|c|c|}
\hline Subset & Clasificador & $\begin{array}{c}\text { Datos } \\
\text { desbalanceados }\end{array}$ & $\begin{array}{c}\text { Balanceo } \\
\text { aplicando } \\
\text { ROS }\end{array}$ & $\begin{array}{c}\text { Balanceo } \\
\text { aplicando } \\
\text { SMOTE }\end{array}$ & $\begin{array}{l}\text { Balanceo } \\
\text { aplicando } \\
\text { ADASYN }\end{array}$ \\
\hline SGB1 & $\mathrm{C} 4.5$ & 0.7894 & 0.7873 & 0.8042 & $0.8162 *$ \\
\hline AIDP-ALL & SVM & 0.7162 & 0.7262 & $0.7750 *$ & $0.7722^{*}$ \\
\hline 20-109 & JRip & 0.7826 & 0.7921 & $0.8102^{*}$ & $0.8215^{*}$ \\
\hline SGB2 & $\mathrm{C} 4.5$ & 0.8729 & 0.8653 & 0.8900 & 0.8949 \\
\hline AMAN-ALL & SVM & 0.8564 & 0.8489 & 0.8490 & $0.8871 *$ \\
\hline $37-92$ & JRip & 0.8608 & 0.8513 & 0.8699 & $0.8949 *$ \\
\hline SGB3 & $\mathrm{C} 4.5$ & 0.8723 & 0.8455 & 0.8795 & 0.8493 \\
\hline AMSAN-ALL & SVM & 0.7948 & 0.7982 & 0.7881 & 0.7827 \\
\hline $59-70$ & JRip & 0.8470 & 0.8358 & 0.8442 & 0.8536 \\
\hline SGB4 & $\mathrm{C} 4.5$ & 0.7808 & 0.7806 & $0.8951 *$ & 0.7331 \\
\hline MF-ALL & SVM & 0.6464 & $0.7590^{*}$ & $0.7516^{*}$ & $0.6991 *$ \\
\hline $13-116$ & JRip & 0.8319 & 0.8440 & $0.8826^{*}$ & 0.7882 \\
\hline
\end{tabular}

Los valores son el promedio de 60 ejecuciones de las curvas ROC utilizando OVA.

modelos obtuvieron diferencia estadísticamente significativa. En el subset SGB3 obtuvo el peor rendimiento. Solo 3 modelos balanceados superaron los datos desbalanceados, sin encontrar diferencia estadísticamente significativa.

En la Tabla 10 se muestran los resultados del ranking para los métodos de balanceo aplicando la técnica de binarización OVA. El algoritmo SMOTE obtuvo el mejor rendimiento con una puntuación promedio de 1.9167. El algoritmo ADASYN obtuvo el segundo lugar con una puntuación promedio de 2.1667. Por último, ROS fue el algoritmo de balanceo con el peor rendimiento, ubicándolo en el tercer lugar con una puntación promedio de 3.0833 .

Respecto a los clasificadores, en la Tabla 11 se observan los resultados del ranking. El clasificador C4.5 obtuvo el primer lugar con una puntuación promedio de 1.2500. El clasificador JRip ocupa el segundo lugar con una puntuación promedio de 1.500. El tercer puesto lo obtuvo el clasificador SVM, con una puntuación promedio de 2.7500 .

Tabla 10. Resultados del ranking por método de balanceo para OVA

\begin{tabular}{lcc}
\hline Método & Ranking & Puntuación promedio \\
\hline SMOTE & 1 & 1.9167 \\
ADASYN & 2 & 2.1667 \\
ROS & 3 & 3.0833 \\
\hline
\end{tabular}

La técnica de binarización OVA fue la que obtuvo los mejores resultados. Se obtuvieron 36 modelos predictivos con datos balanceados. De estos, 12 modelos predictivos obtuvieron diferencia estadísticamente significativa. El algoritmo SMOTE fue el método de balanceo con los mejores resultados. El clasificador JRip fue de acuerdo al ranking el mejor algoritmo.

Tabla 11. Resultados del ranking por clasificador para OVA

\begin{tabular}{lcc}
\hline Clasificador & Ranking & Puntuación promedio \\
\hline C4.5 & 1 & 1.2500 \\
JRip & 2 & 1.5000 \\
SVM & 3 & 2.7500 \\
\hline
\end{tabular}

La técnica de binarización OVO obtuvo el peor rendimiento. Se obtuvieron 54 modelos predictivos con datos balanceados, de estos, 7 modelos predictivos lograron obtener diferencia estadísticamente significativa. El algoritmo ADASYN, obtuvo el mejor rendimiento como método de sobremuestreo. El clasificador C4.5 alcanzó el mejor rendimiento al obtener la menor puntuación promedio.

\section{Conclusiones}

En esta investigación,se realiza una exploración de tres algoritmos de sobremuestreo (ROS, SMOTE y ADASYN), con el objetivo de conocer cuál obtiene el mejor rendimiento; además, conocer si balancear el dataset original mejora el rendimiento de los modelos predictivos realizados con datos desbalanceados. Estos experimentos se realizaron con un dataset real de pacientes diagnosticados con algún subtipo de SGB. Se inicia creando subsets binarios aplicando dos técnicas (OVO y OVA) al dataset original. Se obtienen 10 subsets divididos en: 6 subsets con la técnica OVO 
y 4 subsets con la técnica OVA. Se fracciona cada subset en entrenamiento con $66 \%$ de los datos y $34 \%$ de los datos como prueba. Se sobremuestrea las clases minoritarias de los subsets de entrenamiento aplicando ROS, SMOTE y ADASYN con la finalidad de equilibrar la clase minoritaria con la clase mayoritaria. Una vez balanceados los subsets se aplican tres clasificadores: C4.5, JRip y SVM. Los resultados son el promedio de la curva ROC de 60 ejecuciones. Se aplicó la prueba Wilcoxon a los modelos predictivos obtenidos con datos balanceados que superaron el rendimiento de los modelos con datos desbalanceados para conocer si existe diferencia estadísticamente significativa entre ellos.

La técnica de binarización OVA obtuvo el mejor resultado en comparación con la técnica OVO. Aplicando la técnica OVA se obtuvieron 36 modelos predictivos con datos balanceados, de los cuales 12 obtuvieron diferencia estadísticamente significativa. El mejor algoritmo para balancear los datos fue SMOTE respecto a ROS y ADASYN. El algoritmo SMOTE mejoró el rendimiento de los modelos predictivos de acuerdo con sus características de sobremuestreo. SMOTE agrega instancias de la clase minoritaria extrapolando nuevas instancias en lugar de duplicarlas como lo hace el algoritmo ROS. El algoritmo ROS copia instancias de la clase minoritaria y las agrega al azar duplicando información que puede confundir a los clasificadores. Por otro lado, ADASYN es una variante de SMOTE el cual agrega instancias a la clase minoritaria que son difíciles de aprender, especialmente las que se encuentran en el borde de decisión, este enfoque puede no ser suficiente información para que el clasificador identifique las clases y mejore el resultado. El clasificador C4.5 obtuvo el mejor rendimiento según la puntuación promedio para OVO.

Los resultados demuestran que, balancear los datos mejoran el rendimiento de los modelos predictivos obtenidos con datos desbalanceados. Por otro lado, utilizar algoritmos de aprendizaje automático en problemas de diagnóstico de enfermedades es factible y puede contribuir en la identificación del subtipo de SGB que un paciente contraiga. Como trabajos futuros exploraremos con hibridación de técnicas de sobremuestreo y submuestreo, además de utilizar otros clasificadores.

\section{Referencias}

[1] P. A. van Doorn, "Guillain-Barré syndrome," in Dysimmune Neuropathies. Elsevier, 2020, pp. 5-29. [Online]. Available: https: //doi.org/10.1016/B978-0-12-814572-2.00002-9

[2] A. Tellería-Díaz and D. Calzada-Sierra, "Síndrome de Guillain-Barré," Revista de Neurología, vol. 34, no. 10, pp. 966-976, 2002. [Online]. Available: https://doi.org/10.33588/rn.3410.2001280

[3] E. Alpaydin, Introduction to Machine Learning. MIT press, 2020. [Online]. Available: https://bit.ly/2HvdROG

[4] J. A. Cruz and D. S. Wishart, "Applications of Machine Learning in cancer prediction and prognosis," Cancer Informatics, vol. 2, p. 117693510600200, jan 2006. [Online]. Available: https://doi.org/10.1177/117693510600200030

[5] A. R. Vaka, B. Soni, and S. R. K., "Breast cancer detection by leveraging Machine Learning," ICT Express, may 2020. [Online]. Available: https://doi.org/10.1016/j.icte.2020.04.009

[6] H. Kaur and V. Kumari, "Predictive modelling and analytics for diabetes using a machine learning approach," Applied Computing and Informatics, dec 2018. [Online]. Available: https://doi.org/10.1016/j.aci.2018.12.004

[7] N. P. Tigga and S. Garg, "Prediction of Type 2 Diabetes using Machine Learning classification methods," Procedia Computer Science, vol. 167, pp. 706-716, 2020. [Online]. Available: https://doi.org/10.1016/j.procs.2020.03.336

[8] Z. K. Senturk, "Early diagnosis of parkinson's disease using machine learning algorithms," Medical Hypotheses, vol. 138, p. 109603, may 2020. [Online]. Available: https://doi.org/10.1016/j.mehy.2020.109603

[9] A. Khan and S. Zubair, "An improved multimodal based Machine Learning approach for the prognosis of Alzheimer's disease," Journal of King Saud University - Computer and Information Sciences, apr 2020. [Online]. Available: https://doi.org/10.1016/j.jksuci.2020.04.004

[10] A. Fernández, S. García, M. Galar, R. C. Prati, B. Krawczyk, and F. Herrera, Learning from Imbalanced Data Sets. Springer International Publishing, 2018. [Online]. Available: https://doi.org/10.1007/978-3-319-98074-4

[11] G. Haixiang, L. Yijing, J. Shang, G. Mingyun, H. Yuanyue, and G. Bing, "Learning from class-imbalanced data: Review of methods and applications," Expert Systems with Applications, vol. 73, pp. 220-239, may 2017. [Online]. Available: https://doi.org/10.1016/j.eswa.2016.12.035

[12] A. Fernández, S. García, F. Herrera, and N. V. Chawla, "SMOTE for learning from imbalanced data: Progress and challenges, marking the 15-year anniversary," Journal 
of Artificial Intelligence Research, vol. 61, pp. 863-905, apr 2018. [Online]. Available: https://doi.org/10.1613/jair.1.11192

[13] K. Napierala and J. Stefanowski, "Types of minority class examples and their influence on learning classifiers from imbalanced data," Journal of Intelligent Information Systems, vol. 46, no. 3, pp. 563-597, jul 2015. [Online]. Available: https://doi.org/10.1007/s10844-015-0368-1

[14] J. Canul-Reich, J. Frausto-Solís, and J. Hernández-Torruco, "A predictive model for Guillain-Barré syndrome based on single learning algorithms," Computational and Mathematical Methods in Medicine, vol. 2017, pp. 1-9, 2017. [Online]. Available: https://doi.org/10.1155/2017/8424198

[15] J. Canul-Reich, J. Hernández-Torruco, O. ChávezBosquez, and B. Hernández-Ocaña, "A predictive model for Guillain-Barré syndrome based on ensemble methods," Computational Intelligence and Neuroscience, vol. 2018, pp. 1-10, 2018. [Online]. Available: https://doi.org/10.1155/2018/1576927

[16] J. Hernández-Torruco, J. Canul-Reich, J. FraustoSolís, and J. J. Méndez-Castillo, "Feature selection for better identification of subtypes of Guillain-Barré syndrome," Computational and Mathematical Methods in Medicine, vol. 2014, pp. 1-9, 2014. [Online]. Available: https://doi.org/10.1155/2014/432109

[17] A. Fernández, S. del Río, N. V. Chawla, and F. Herrera, "An insight into imbalanced big data classification: Outcomes and challenges," Complex \& Intelligent Systems, vol. 3, no. 2, pp. 105-120, 2017. [Online]. Available: https://doi.org/10.1007/s40747-017-0037-9

[18] N. V. Chawla, K. W. Bowyer, L. O. Hall, and W. P. Kegelmeyer, "SMOTE: Synthetic minority over-sampling technique," Journal of Artificial Intelligence Research, vol. 16, pp. 321-357, jun 2002. [Online]. Available: https://doi.org/10.1613/jair.953

[19] H. He, Y. Bai, E. A. García, and S. Li, "ADASYN: Adaptive synthetic sampling approach for imbalanced learning," in 2008 IEEE International Joint Conference on Neural Networks (IEEE World Congress on Computational Intelligence). IEEE, jun 2008. [Online]. Available: https://doi.org/10.1109/IJCNN.2008.4633969

[20] S. Ruggieri, "Efficient C4.5 [classification algorithm]," IEEE Transactions on Knowledge and Data Engineering, vol. 14, no. 2, pp. 438-444, 2002. [Online]. Available: https://doi.org/10.1109/69.991727

[21] T. S. Furey, N. Cristianini, N. Duffy, D. W. Bednarski, M. Schummer, and D. Haussler, "Support Vector Machine classification and validation of cancer tissue samples using microarray expression data," Bioinformatics, vol. 16, no. 10, pp. 906-914, 2000. [Online]. Available: https: //doi.org/10.1093/bioinformatics/16.10.906

[22] A. Rajput, R. P. Aharwal, M. Dubey, S. Saxena, and M. Raghuvanshi, "J48 and JRip rules for e-governance data," International Journal of Computer Science and Security (IJCSS), vol. 5, no. 2, p. 201, 2011. [Online]. Available: https://bit.ly/3jt2jrY

[23] R. Kannan and V. Vasanthi, "Machine learning algorithms with ROC curve for predicting and diagnosing the heart disease," in Soft Computing and Medical Bioinformatics. Springer Singapore, jun 2018, pp. 63-72. [Online]. Available: https://doi.org/10.1007/978-981-13-0059-2_8

[24] A. Fernández, V. López, M. Galar, M. J. del Jesús, and F. Herrera, "Analysing the classification of imbalanced data-sets with multiple classes: Binarization techniques and ad-hoc approaches," Knowledge-Based Systems, vol. 42, pp. 97-110, apr 2013. [Online]. Available: https://doi.org/10.1016/j.knosys.2013.01.018

[25] A. D. Pozzolo, O. Caelen, and G. Bontempi, unbalanced: Racing for Unbalanced Methods Selection, 2015, R package version 2.0. [Online]. Available: https://doi.org/10.1007/978-3-642-41278-3_4

[26] L. Torgo, Data Mining with R, learning with case studies. Chapman and Hall/CRC, 2010. [Online]. Available: https://bit.ly/3jtkeyV

[27] P. Branco, R. P. Ribeiro, and L. Torgo, "UBL: an R package for utility-based learning," CoRR, vol. abs/1604.08079, 2016. [Online]. Available: https://bit.ly/35yeFtU

[28] I. H. Witten, E. Frank, M. A. Hall, and C. Pañ, Data Mining, Practical Machine Learning Tools and Techniques, Elsevier, Ed. Morgan Kaufmann, 2017. [Online]. Available: https://doi.org/10.1145/507338.507355

[29] D. Meyer, E. Dimitriadou, K. Hornik, A. Weingessel, and F. Leisch, e1071: Misc Functions of the Department of Statistics, Probability Theory Group (Formerly: E1071), TU Wien, 2018, $\mathrm{R}$ package version 1.7-0. [Online]. Available: https://bit.ly/3mm1d3s 
[30] A. S. Hussein, T. Li, W. Y. Chubato, and K. Bashir, "A-SMOTE: A new preprocessing approach for highly imbalanced datasets by improving SMOTE," International Journal of Computational Intelligence Systems, 2019. [Online]. Available: https://bit.ly/3mhotiT 


\title{
DISEÑO Y CONSTRUCCIÓN DE UN REACTOR DISCONTINUO CON RECIRCULACIÓN EXTERNA PARA OBTENER BIODIÉSEL A PARTIR DE ACEITE DE FRITURA EN CONDICIONES SUBCRÍTICAS
}

\author{
DESIGN AND CONSTRUCTION OF A BATCH \\ REACTOR WITH EXTERNAL RECIRCULATION \\ TO OBTAIN BIODISEL FROM RESIDUAL OIL \\ FRYING UNDER SUBCRITICAL CONDITIONS
}

\author{
Cristian Fabián Pérez-Salinas ${ }^{1, *}$, Diego Fernando Núnez-Núñez ${ }^{1}$, \\ Herminia del Rosario Sanaguano-Salguero ${ }^{2}$, Luis Fernando Sánchez-Quinchuela ${ }^{3}$
}

Recibido: 23-05-2020, Revisado: 06-08-2020, Aprobado tras revisión: 25-09-2020

\section{Resumen}

Se diseña y construye un reactor discontinuo para obtener biodiésel a partir de aceite de fritura en condiciones subcríticas con la intención de reducir el tiempo de reacción al mínimo posible. El proceso de diseño se centra en la selección del material y la verificación de su resistencia mediante un análisis FEM a partir de un diseño experimental DOE. Se consideran tres niveles de presión, temperatura y espesor de pared, respectivamente, y un factor categórico material a dos niveles. Los resultados obtenidos permiten determinar que el material apropiado para la manufactura del reactor es acero inoxidable 304 con un factor de seguridad de diseño de 1 . Para el proceso de construcción del sistema es necesario también la selección de todos los componentes complementarios. Las pruebas finales de funcionamiento muestran que es posible obtener el biocombustible en el reactor discontinuo con un grado de conversión del $88 \%$ de manera segura en un rango de 5 a 8 minutos.

Palabras clave: biodiésel, reactor discontinuo, condiciones subcríticas, DOE, elementos finitos

\section{Abstract}

A batch reactor was designed and built to obtain biodiesel from frying oil under sub-critical conditions, with the purpose of reducing the reaction time to the minimum possible. The design process is focused on the selection of the material and the verification of its resistance by means of a FEM analysis from a Design of Experiments (DOE). Three levels of pressure, temperature and wall thickness, respectively, and a material categorical factor at two levels were considered. The results obtained were that the appropriate material for manufacturing the reactor is 304 stainless steel with a design safety factor of 1 . For constructing the system it was also necessary to select all the complementary components. The final operation tests showed that it is possible to safely obtain the biofuel in the batch reactor with a degree of conversion $88 \%$, in a range of 5 to 8 minutes.

Keywords: Biodiesel, Discontinuous reactor, subcritical conditions, DOE, Finite elements.

\footnotetext{
$\overline{1, *}$ Docente investigador, Ingeniería Mecánica, Universidad Técnica de Ambato, Ecuador.

Autor para correspondencia : cf.perez@uta.edu.ec. (D) http://orcid.org/0000-0003-4031-5464

(D) http://orcid.org/0000-0001-5248-4084

${ }^{2}$ Docente investigador, Ciencias Ambientales, Universidad Estatal de Bolívar, Ecuador.

(D) http://orcid.org/0000-0002-2885-1515

${ }^{3}$ Profesor, Mecánica Industrial, Instituto Superior Tecnológico Guayaquil, Ecuador.

(D) http://orcid.org/0000-0002-4238-7376
}

Forma sugerida de citación: Pérez-Salinas, C. F.; Núnez-Núñez, D. F.; Sanaguano-Salguero, H, R. y SánchezQuinchuela, L. F. (2021). «Diseño y construcción de un reactor discontinuo con recirculación externa para obtener biodiésel a partir de aceite de fritura en condiciones subcríticas». INGENIUS. N. ${ }^{\circ} 25$, (enero-junio). pp. 32-40. DOI: https://doi.org/10.17163/ings.n25.2021.03. 


\section{Introducción}

La mayoría de los países del mundo están preocupados por reducir las emisiones de gases de efecto invernadero, aumentar las nuevas fuentes alternativas a los combustibles fósiles y recuperar el territorio deforestado con vegetación cultivable o no cultivable [1]. Para este propósito, la producción de biocombustibles es una alternativa tangible [2-4]. Se espera que el aumento en la producción de biocombustibles no solo contribuya a la conservación del medioambiente, sino también al desarrollo económico y social de los países productores [5].

Las técnicas convencionales para producir biodiésel utilizan reactores. Un reactor químico es un dispositivo complejo en el cual la transferencia de calor y masa, la difusión y la fricción pueden ocurrir junto con la reacción química bajo dispositivos de control y seguridad. Existen diferentes tipos de reactores según la forma de obtención, el tipo de flujo interno y las fases que albergan [6-9]. En general, se busca conocer el tamaño y el tipo de reactor, así como el método de operación necesario para satisfacer la obtención del producto final [10].

Uno de los parámetros importantes que se ha pretendido mejorar es el tiempo de obtención del biodiésel. En estudios previos se encuentra reportes de tiempos que van desde los 20 a los 180 minutos [11], particularmente en aceites de residuos de cocina el tiempo de obtención es de 90 minutos.

En los últimos años, la atención se ha centrado en la búsqueda de materias primas diferentes de los aceites vegetales como la soja, la palma, etc. Esto se debe principalmente al costo de obtener la materia prima, que es aproximadamente el $70 \%$ del costo total de obtener biodiésel. [6-8]. El aceite de fritura es otra fuente de materia prima, su ventaja sobre otras materias primas es que tiene la categoría de residuos. Esta característica le da una gran viabilidad porque contribuye a la reducción de la contaminación ambiental.

En Latinoamérica, el residuo de aceite de fritura tiene potencialidad de uso como fuente primaria para la producción de biodiésel [12]. Existe una industria amplia de comida rápida y tradicional que usa el aceite de fritura, pero después de su uso se convierte en un desecho. Ejemplos como en Colombia, el $35 \%$ de 162 millones de litros de la producción anual de aceite de cocina se convierte en residuo desechado en alcantarillas [13], España produce 150 millones de litros anuales de aceite vegetal usado [14].

Por otra parte, en los sectores industrial, educativo y doméstico, existen innumerables productos y sistemas desarrollados para cumplir una función específica. Todos ellos emplean materiales de ingeniería seleccionados para conferir un rendimiento óptimo. El proceso de obtención de un producto pasa por tres macroprocesos: diseño, fabricación y pruebas de fun- cionamiento. La fase de diseño comienza con la determinación de los requisitos y restricciones para llevar a cabo posteriormente un proceso de selección de material [15].

En el desarrollo ingenieril de los últimos años, se han utilizado dos métodos para la selección de materiales: el tradicional y el gráfico. El primero se basa en el conocimiento y la experiencia del ingeniero, el segundo en mapas gráficos de materiales de ingeniería organizados por sus propiedades físico-mecánicas [16].

El método gráfico se desarrolló como un soporte muy importante en la fase de concepción y desarrollo del producto $[17,18]$. El método gráfico de Michael Ashby ha tenido mayor utilidad en los últimos años. Es una metodología que sirve de guía en la selección de los materiales, contemplando los atributos que se relacionan entre sí mediante tablas de selección gráfica $[18,19]$. Se utilizan índices de rendimiento, estos se refieren a agrupaciones de las propiedades de los materiales con el fin de maximizarlos o minimizarlos de acuerdo con el requisito específico. Se derivan de la función objetivo del sistema y se expresan mediante ecuaciones matemáticas.

Varios autores informan el uso de la técnica de Análisis de Elementos Finitos (FEA) para el diseño y verificación de reactores químicos sometidos a modelado multifísico [20-23]. En estudios, como [24-26], mencionan que el diseño paramétrico proporciona a los sistemas mecánicos la posibilidad de sintetizar, simplificar y economizar el proceso de diseño, lo que permite amplificar y explorar las posibilidades de solución.

En el presente estudio, se llevó a cabo un proceso de diseño y construcción de un reactor discontinuo con recirculación externa para obtener biodiésel del aceite de fritura en condiciones subcríticas, con el propósito de reducir el tiempo de producción dentro de un marco controlado y seguro que cumpla con los requisitos de ingeniería. Para el efecto, se procedió a la selección de materiales y la verificación de la resistencia bajo el régimen de tensión utilizando la técnica de elementos finitos.

\section{Materiales y métodos}

Se siguió la siguiente metodología: selección de materiales, diseño mediante el análisis de elementos finitos (FEA), construcción del sistema y pruebas de funcionamiento. Se resalta el hecho que el diseño del reactor se inició con observación de diseños previos para concertarle en un diseño completamente nuevo que permita la reducción del tiempo de obtención del biodiésel.

\subsection{Selección de materiales}

Se utilizó el método gráfico de Ashby, con la ayuda del software educativo CES EDUPACK. Se definió la 
función requerida, el objetivo y las restricciones en el reactor (Tabla 1). A partir de la ley de Hooke que expresa la tensión en la zona elástica $(\sigma)$ es igual al módulo de Young (E) por su deformación $(\epsilon)$ y la fórmula de la masa $(\mathrm{m})$ de acuerdo con su volumen y densidad $(\rho)$, se determinó el índice de rendimiento (Ecuación 1).

Tabla 1. Definición de los requerimientos del diseño

\begin{tabular}{cc}
\hline Definición & Detalle \\
\hline Función & Alojar proceso termoquímico \\
Objetivo & $\begin{array}{c}\text { Minimizar la masa del reactor }(\mathrm{r}) \text { y maximizar } \\
\text { la resistencia mecánica }(\mathrm{s})\end{array}$ \\
& Soportar temperaturas mínimas de $160{ }^{\circ} \mathrm{C}$ \\
& y máxima de $400{ }^{\circ} \mathrm{C}$ \\
Restricciones & Soportar presión mínima de 250 psi y máxima \\
& de 3000 \\
& Resistencia a procesos oxidativo corrosivo \\
Bajo costo \\
Buena maquinabilidad y soldable
\end{tabular}

$$
\begin{aligned}
m & =\frac{F}{\epsilon \cdot E} \cdot L \cdot \rho=F \cdot L \cdot\left[\frac{\rho}{\sigma}\right] \\
& \Rightarrow \text { Indice_desempeño }=\frac{\rho}{\sigma}
\end{aligned}
$$

Tomando todas las funciones y restricciones iniciales para el reactor, se obtuvo la posibilidad de 600 alternativas de material de un total de 3900 opciones. El ajuste de la selección se realizó de acuerdo con las condiciones específicas de servicio (resistencia mecánica alta, buen comportamiento a temperatura elevadas y resistente a elementos químicos), disponibilidad local del material al menor costo posible y una densidad alta que permita una estabilidad del sistema al movimiento (Figura 1).

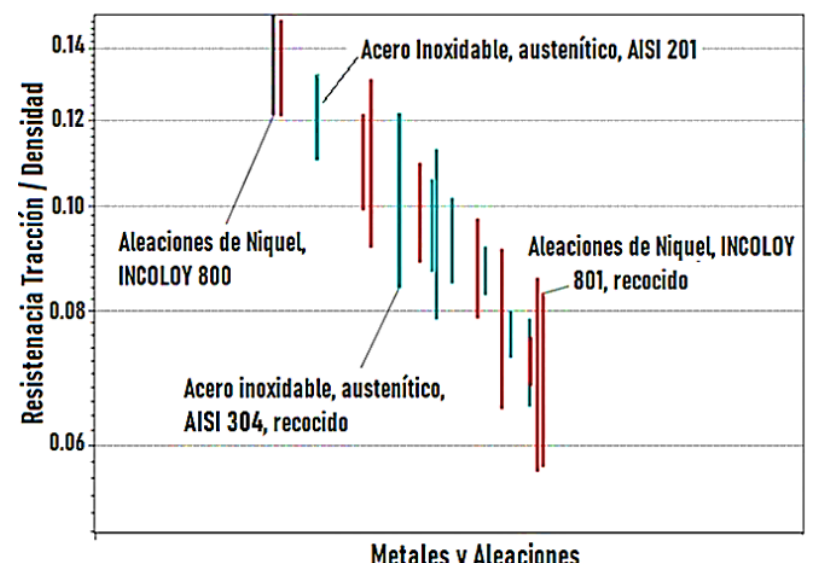

Figura 1. Gráfica de selección de materiales esfuerzodensidad [18]

Las variables de entrada fueron: precio máximo de 10 USD, densidad entre 5000 y $8000 \mathrm{~kg} / \mathrm{m}^{3}$, temperatura de servicio de $500{ }^{\circ} \mathrm{C}$, resistencia a sustancias alcalinas y sustancias con $\mathrm{pH}$ superior a 7 , capacidad de moldeo y maquinabilidad que dan como resultado 18 posibilidades de material enmarcadas en tres grupos: aceros inoxidables austeníticos, aceros inoxidables austeníticos recocidos y aleaciones de níquel.

\subsection{Análisis por elementos finitos (FEA)}

Una vez realizada la selección de materiales para el reactor, se selecciona el acero AISI 304 y la aleación de níquel para ingresar en diseño experimental FEA. Por otra parte, las dimensiones para el reactor discontinuo se plantearon para que obtenga una capacidad de recirculación continua de 1 litro.

Se planteó un diseño factorial completo donde se tomó en cuenta los tres factores principales (restricciones/requerimientos) del reactor; la presión, temperatura y espesor de pared en tres niveles respectivamente. También se consideró una variable categórica (material) en dos niveles. La Tabla 2 muestra el diseño experimental. Los rangos de los valores de presión y temperatura se basaron en estudios de obtención de biodiésel en condiciones subcríticas y supercríticas.

Tabla 2. Diseño de experimento

\begin{tabular}{cccc}
\hline \multirow{2}{*}{ Factores } & \multicolumn{3}{c}{ Niveles } \\
\cline { 2 - 4 } & Bajo & Medio & Alto \\
\hline Presión (Mpa) & 1,72 & 4,83 & 20,7 \\
Temperatura $\left({ }^{\circ} \mathrm{C}\right)$ & 160 & 200 & 400 \\
Espesor de pared (mm) & 1,5 & 2 & 3 \\
Material & Acero AISI 504 & Aleación Níquel & - \\
\hline
\end{tabular}

Se llevó a cabo un análisis termomecánico mediante elementos finitos utilizando un modelo 2D simétrico multiparamétrico realizado en Ansys APDL para evaluar la resistencia mecánica del reactor a las condiciones internas de presión y temperatura especificadas en el diseño de los experimentos. Se utilizó una cuarta parte de la sección transversal debido a los dos planos de simetría existentes en el modelo, Figura 2. Para los dos materiales utilizados en este estudio se definió un modelo isotrópico lineal elástico de material, sus propiedades se muestran en la Tabla 3.

Tabla 3. Propiedades mecánicas y térmicas para el modelo de material isotrópico elástico lineal

\begin{tabular}{ccc}
\hline \multirow{2}{*}{ Propiedades mecánicas y térmicas } & \multicolumn{2}{c}{ Material } \\
\cline { 2 - 3 } & AISI 304 & Ni 800 \\
\hline Módulo de elasticidad $(\mathrm{GPa})$ & 193 & 196 \\
Coeficiente de Poisson & 0,3 & 0,34 \\
Esfuerzo de fluencia $(\mathrm{MPa})$ & 220 & 335 \\
Densidad $\left(\mathrm{kg} / \mathrm{m}^{3}\right)$ & 7850 & 7940 \\
Temperatura de servicio $\left({ }^{\circ} \mathrm{C}\right)$ & 850 & 816 \\
Conductividad $\left(\mathrm{W} / \mathrm{kg}^{-} \mathrm{K}\right)$ & 16,2 & 11,5 \\
Calor específico $\left(\mathrm{J} / \mathrm{kg}^{\circ} \mathrm{C}\right)$ & 500 & 460 \\
Coeficiente de expansión térmica $\left(\mathrm{x} 10^{6}{ }^{\circ} \mathrm{C}^{-1}\right)$ & 17 & 12 \\
\hline
\end{tabular}




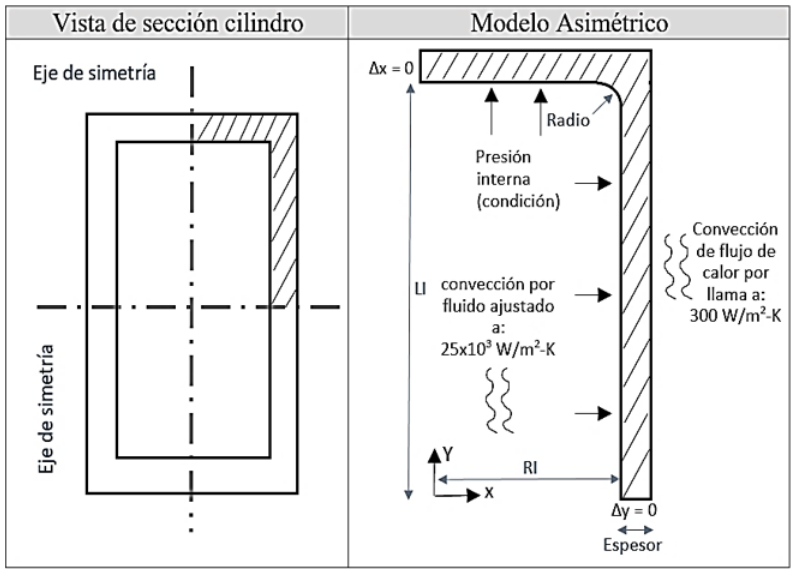

Figura 2. Geometría y condiciones límite de un modelo de reactor asimétrico de un cuarto de eje para un análisis termomecánico

El modelo se discretizó con elementos cuadriláteros PLANE182 en su versión axisimétrica; el tamaño del elemento para todos los casos se estableció en 1/5 del espesor de la pared del cilindro, ya que con este valor se aseguró una convergencia menor al $5 \%$. El modelo consta de 1830 nodos y 1520 elementos. Las condiciones límite para el análisis termomecánico estacionario se muestran en la Figura 2.

Se realizaron dos tipos de análisis de elementos finitos: uno térmico estacionario con las condiciones de temperatura y convección especificadas para obtener los desplazamientos y esfuerzos térmicos y luego un análisis estructural estático con las condiciones de frontera indicadas de tal manera que ambos estudios se acoplaron en un análisis multifísico. La validación del modelo de elementos finitos del primer tipo de análisis se realizó mediante la teoría de recipientes a presión de paredes delgadas (TWPVt) y se evaluaron las tensiones circunferenciales o tangenciales (SZ) y longitudinales o axiales (SY), porque esta teoría no tenga en cuenta las concentraciones de tensión, tales como cubiertas, agujeros o cambios abruptos en la sección transversal. En el segundo análisis de elementos finitos se evaluó la influencia de la presión y la temperatura y se compararon los resultados de la tensión de Von Misses equivalente con la tensión de fluencia de cada material para determinar el factor de seguridad del reactor.

$$
\sigma_{e}=\sqrt{\left(\sigma_{x}+\sigma_{y}\right)-3\left(\sigma_{x} \sigma_{y}-\tau_{x y}^{2}\right)}
$$

Donde:

$$
\begin{aligned}
& \sigma_{e}=\text { Esfuerzo de Von Misses } \\
& \sigma_{x}=\text { Esfuerzo normal en el eje X } \\
& \sigma_{y}=\text { Esfuerzo normal en el eje Y } \\
& \tau_{x y}^{2}=\text { Esfuerzo cortante en el plano XY }
\end{aligned}
$$

Según la teoría de los recipientes a presión de paredes delgadas (relación diámetro interno / espesor, mayor o igual a 10), existen dos tensiones principales que son función de la presión interna $(p)$, el radio interno $(r)$ y el espesor de pared $(t)$. Estas son tensiones longitudinales o axiales y circunferenciales o tangenciales, sus fórmulas de cálculo son (3) y (4) respectivamente:

Tensión longitudinal o axial (SY):

$$
\sigma_{1}=\frac{p r}{2 t}
$$

Tensión circunferencial o tangencial (SZ):

$$
\sigma_{t}=\frac{p r}{t}
$$

\subsection{Construcción del sistema}

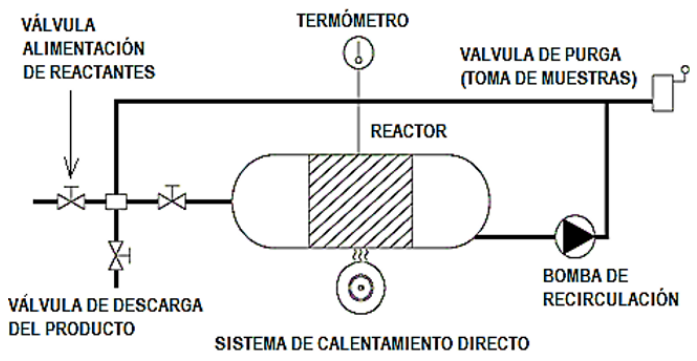

(a)

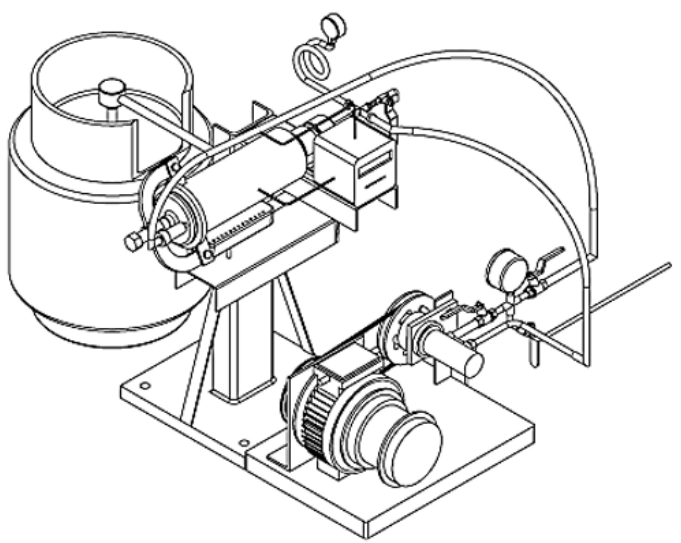

(b)

Figura 3. Reactor discontinuo a) Esquema del sistema, b) Configuración 3D

El reactor discontinuo (Figura 3) está constituido por un tanque, un tubo de circulación y una bomba de recirculación para la agitación de los reactivos. El tanque consta de un tubo cilíndrico de $280 \mathrm{~mm}$ de longitud, un diámetro interno de $71 \mathrm{~mm}$ y un espesor de pared de acuerdo con el espesor mínimo resultado del diseño (análisis FEM). El tanque está acoplado con un tubo de $1000 \mathrm{~mm}$ de largo por $12 \mathrm{~mm}$ de diámetro y una bomba de alta presión que gira a 250 rpm para recirculación y agitación. El reactor como el tubo se fabricará del material adecuado estudiado en 
el apartado 2.2 y que esté disponible al menor costo. El flujo de carga $(3 \mathrm{l} / \mathrm{min})$ es impulsado por un motor de la marca Motovario TXF005. Un termopar conectado al controlador de temperatura y un manómetro para medir la presión interna del recipiente se ubicaron en la tapa del reactor. La cubierta consta de una brida con junta de teflón. En la parte superior del reactor se colocaron dos medidores de presión Problock de 0 a 150 PSI. La temperatura del reactor es registrada por un controlador de temperatura Syscon AKC CB100.

\subsection{Pruebas de funcionamiento}

Las pruebas se llevaron a cabo a temperaturas de 160 , 180 y $200{ }^{\circ} \mathrm{C}$, con relaciones molares de metanol-aceite de $6: 1$ y $9: 1$, porcentaje de catalizador $(\mathrm{NaOH})$ de 0,5 y $1 \%$ y para un tiempo de reacción de 5 a 10 minutos para verificar la funcionalidad del reactor discontinuo con capacidad de recirculación continua de 1 litro, alcanzando presiones de 250 psi (condición subcrítica). Para la obtención del biodiésel crudo se someterá a una destilación y lavado con agua atomizada hasta que el agua de lavado se obtenga limpia. Una vez que se eliminen los productos secundarios, el biodiésel se calentará $105{ }^{\circ} \mathrm{C}$ durante 10 minutos para secar o eliminar el agua y los residuos de metanol. Una vez enfriado, se obtuvo un volumen de biodiésel refinado.

\section{Resultados y discusión}

\subsection{Resultados numéricos y analíticos}

Los resultados del análisis estructural estático se muestran en la Tabla 4. El acero inoxidable AISI 304 y la aleación de níquel Ni 800 tienen un módulo de elasticidad y una relación de Poisson muy similares, lo que significa que los resultados del esfuerzo (SY) y circunferencial (SZ) prácticamente no varían, lo que cambia es el factor de seguridad, definido como la relación entre el esfuerzo de fluencia del material y el esfuerzo equivalente máximo alcanzado. Es importante tener en cuenta que en este análisis el factor de seguridad solo se obtuvo con las tensiones circunferenciales y longitudinales, lo que no necesariamente representa un estado tensional completo del punto analizado. Según los resultados del modelo de elementos finitos, concuerda satisfactoriamente con la teoría de los recipientes a presión de pared delgada. El error máximo fue de aproximadamente $4,2 \%$.

\subsection{Influencia del reactor de presión interna y temperatura}

El estudio del acoplamiento termomecánico de una cuarta parte de la sección transversal del reactor se utilizó para determinar la influencia de la presión interna y la temperatura. Las Tablas 5, 6 y 7 muestran la influencia de la presión interna para una condición de temperatura constante de $160^{\circ} \mathrm{C}$. La leyenda del color se separó para el acero inoxidable AISI 304 y la aleación de níquel Ni800, ya que tienen un límite elástico diferente. El color rojo en la Tabla 4 significa que el material excede el límite elástico y existe la posibilidad de deformaciones permanentes que conducen a la falla del biorreactor. La escala de deformación con respecto a la condición no deformada es la misma para cada caso.

Tabla 4. Comparación de las tensiones longitudinales $\sigma_{l}(\mathrm{SY})$ y circunferenciales $\sigma_{t}(\mathrm{SZ})$ del análisis de elementos finitos (FEA) y la teoría de los recipientes a presión de pared delgada (TWPVt)

\begin{tabular}{|c|c|c|c|c|c|c|c|c|c|c|c|}
\hline $\operatorname{Exp}$ & $\begin{array}{l}\text { Presión } \\
\text { (psi) }\end{array}$ & $\begin{array}{c}\text { DOE } \\
\text { Espesor } \\
(\mathrm{mm})\end{array}$ & Material & $\begin{array}{c}\text { FEA } \\
\text { SY }\end{array}$ & $\begin{array}{c}\text { TWPVt } \\
\sigma_{l}\end{array}$ & $\begin{array}{c}\text { Error } \\
\%\end{array}$ & $\begin{array}{c}\text { Factor } \\
\text { seguridad }\end{array}$ & $\begin{array}{c}\text { FEA } \\
\text { SZ }\end{array}$ & $\begin{array}{c}\text { TWPVt } \\
\sigma_{t}\end{array}$ & $\begin{array}{c}\text { Error } \\
\%\end{array}$ & $\begin{array}{c}\text { Factor } \\
\text { seguridad }\end{array}$ \\
\hline 1 & 250 & 1,5 & AISI 304 / Ni 800 & 2857 & 2917 & 2,1 & $10,5 / 15,8$ & 5960 & 5834 & 2,1 & $5,0 / 7,6$ \\
\hline 2 & 250 & 2 & AISI 304 / Ni 800 & 2127 & 2188 & 2,8 & $14,1 / 21,2$ & 4502 & 4375 & 2,8 & $6,7 / 10,0$ \\
\hline 3 & 250 & 3 & AISI 304 / Ni 800 & 1399 & 1458 & 4,1 & $21,4 / 32,2$ & 3045 & 2917 & 4,2 & $9,9 / 14,8$ \\
\hline 4 & 700 & 1,5 & AISI 304 / Ni 800 & 8066 & 8236 & 2,1 & $3,7 / 5,6$ & 16827 & 16472 & 2,1 & $1,8 / 2,7$ \\
\hline 5 & 700 & 2 & AISI 304 / Ni 800 & 6003 & 6177 & 2,8 & $5,0 / 7,5$ & 12711 & 12354 & 2,8 & $2,4 / 3,5$ \\
\hline 6 & 700 & 3 & AISI 304 / Ni 800 & 3951 & 4118 & 4 & $7,6 / 11,4$ & 8597 & 8236 & 4,2 & $3,5 / 5,2$ \\
\hline 7 & 3000 & 1,5 & AISI 304 / Ni 800 & 34614 & 35346 & 2,1 & $0,9 / 1,3$ & 72216 & 70692 & 2,1 & $0,4 / 0,6$ \\
\hline 8 & 3000 & 2 & AISI 304 / Ni 800 & 25784 & 26510 & 2,7 & $1,2 / 1,7$ & 54552 & 53019 & 2,8 & $0,5 / 0,8$ \\
\hline 9 & 3000 & 3 & AISI 304 / Ni 800 & 16956 & 17673 & 4,1 & $1,8 / 2,7$ & 36898 & 35346 & 4,2 & $0,8 / 1,2$ \\
\hline
\end{tabular}


Tabla 5. Análisis numérico de la influencia de la presión interna del reactor

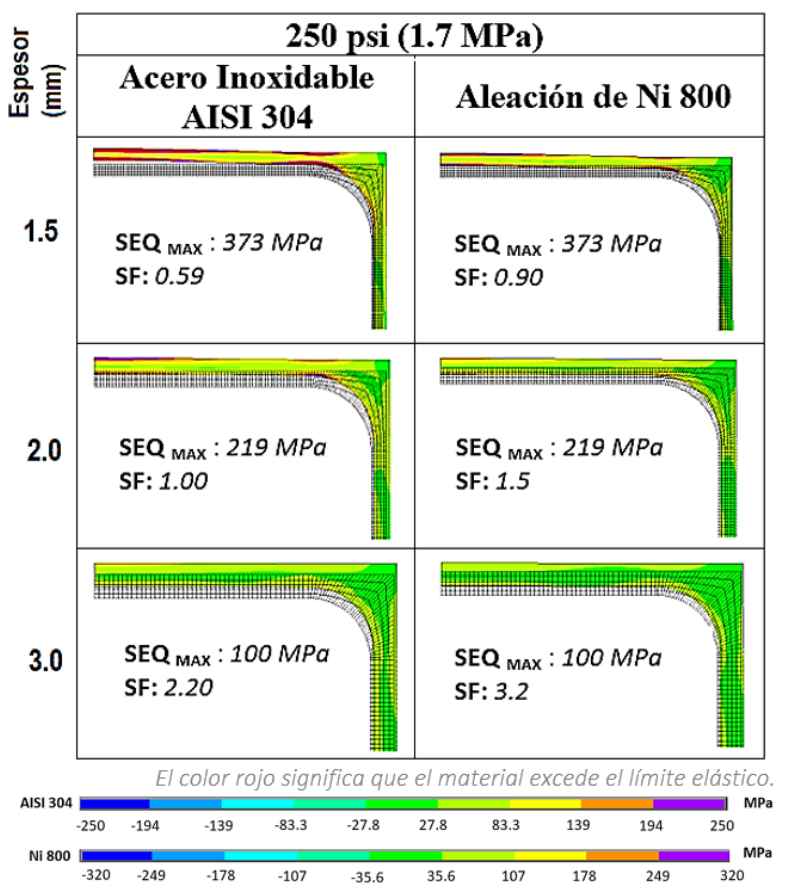

Temperatura constante $=160{ }^{\circ} \mathrm{C}$ y presión interna 250 psi (1.7 MPa)

Tabla 6. Análisis numérico de la influencia de la presión interna del reactor

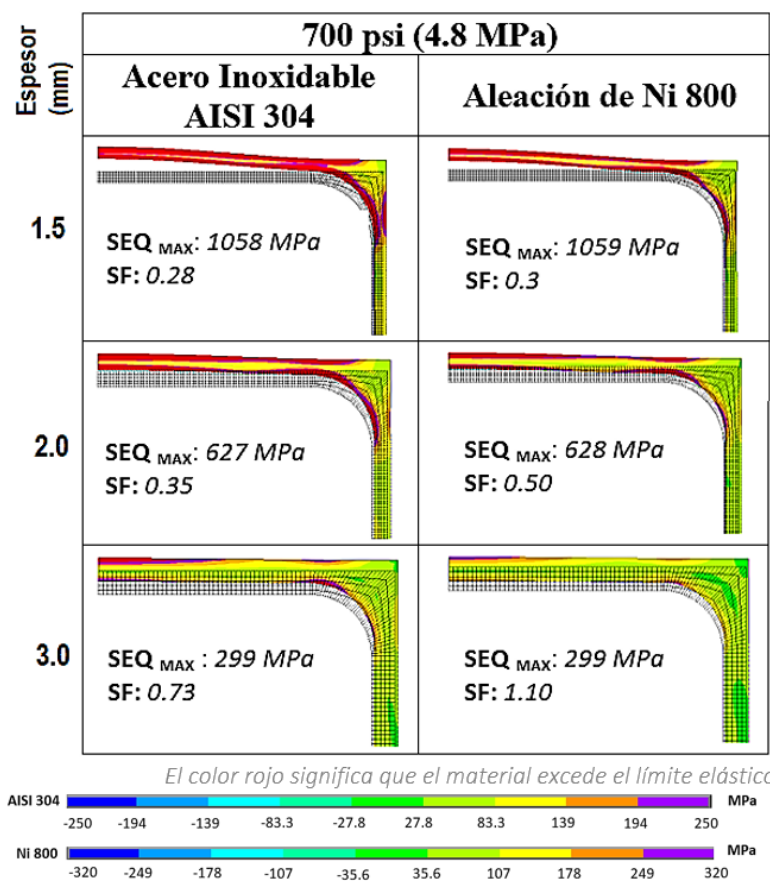

Temperatura constante $=160{ }^{\circ} \mathrm{C}$ y presión interna 700 psi (4,8 MPa)

El análisis de elementos finitos muestra resultados similares de tensión equivalente de Von Misses para ambos materiales, debido a que el módulo de elasticidad y la relación de Poisson son muy similares; sin embargo, los factores de seguridad son diferentes debido a que existe una diferencia entre su límite de elasticidad de alrededor de $100 \mathrm{MPa}$. El esfuerzo máximo de Von Misses alcanzado fue mayor para espesores de 1,5 $\mathrm{mm}$ de pared del reactor y mínimos para espesores de $3 \mathrm{~mm}$. Para una presión interna de 250, 700 y 3000 psi, el esfuerzo máximo de Von Misses fue de 373, 1058 y $4555 \mathrm{MPa}$, respectivamente.

Tabla 7. Análisis numérico de la influencia de la presión interna del reactor

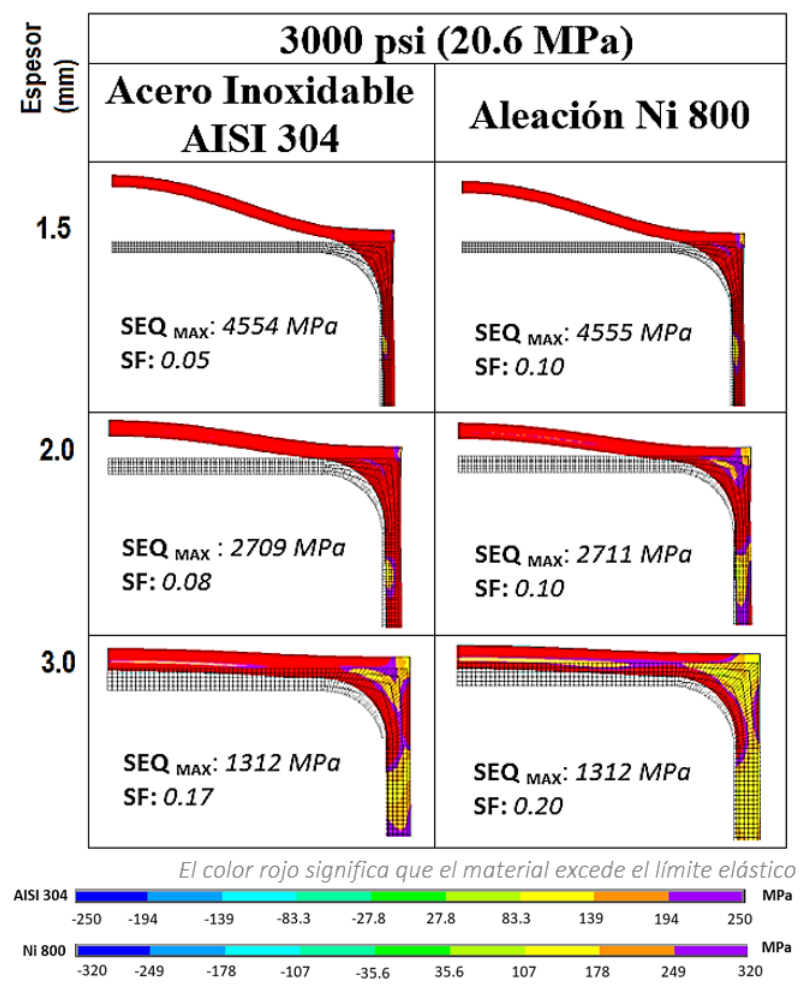

Temperatura constante $=160{ }^{\circ} \mathrm{C}$ y presión interna 3000 psi (20,6 MPa)

Con las condiciones del modelo de elementos finitos axisimétricos desarrollado en este estudio, se verifica que para el caso del acero inoxidable AISI 304 el diseño solo es seguro hasta condiciones subcríticas de presión de 250 psi y un espesor mínimo de $2 \mathrm{~mm}$ donde un factor de seguridad de 1,00 fue obtenido. El factor de seguridad se calcula como la relación entre el esfuerzo elástico del material y el esfuerzo equivalente máximo alcanzado.

Para la aleación de níquel Ni800, la presión interna se puede aumentar hasta 700 psi y un grosor de $3 \mathrm{~mm}$, donde el factor de seguridad es 1,10. Para condiciones de presión interna supercrítica (3000 psi), los factores de seguridad son inferiores a 1,00 en todos los casos, lo que indica que el material tiene una probabilidad muy alta de falla. Para esta condición, la pared de espesor del reactor debe incrementarse drásticamente. 
Las Tablas 8, 9 y 10 muestran la influencia de la temperatura para una condición de presión interna constante de 250 psi.

Tabla 8. Análisis de la influencia de la temperatura del reactor

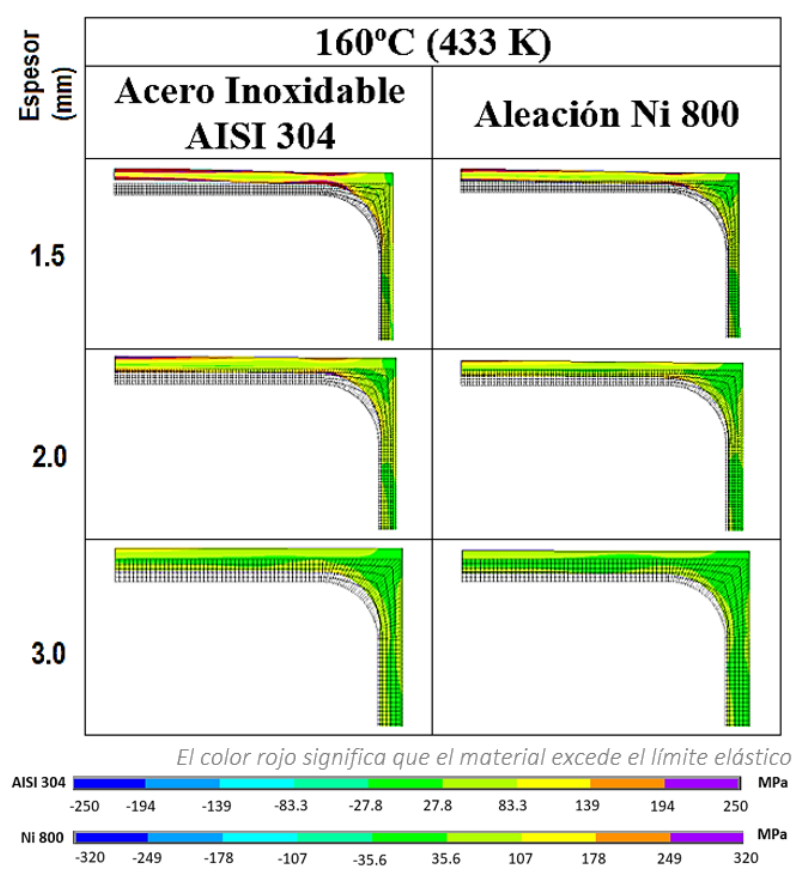

Presión constante $=250$ psi, temperaturas de $160{ }^{\circ} \mathrm{C}$

Tabla 9. Análisis de la influencia de la temperatura del reactor

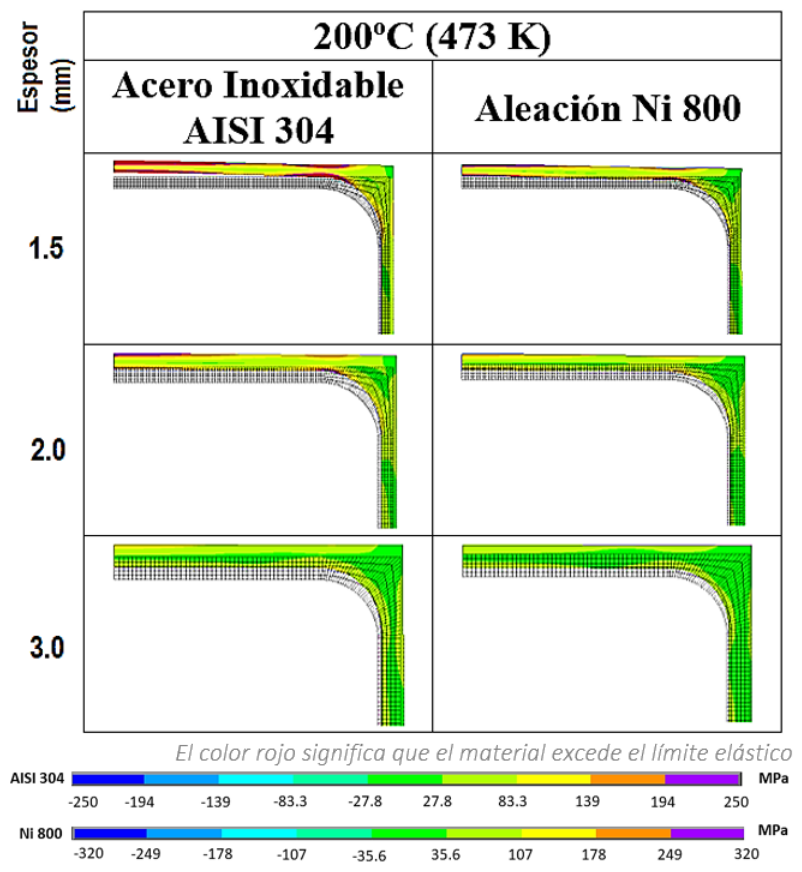

Presión constante $=250$ psi, temperaturas de $200{ }^{\circ} \mathrm{C}$
Tabla 10. Análisis de la influencia de la temperatura del reactor

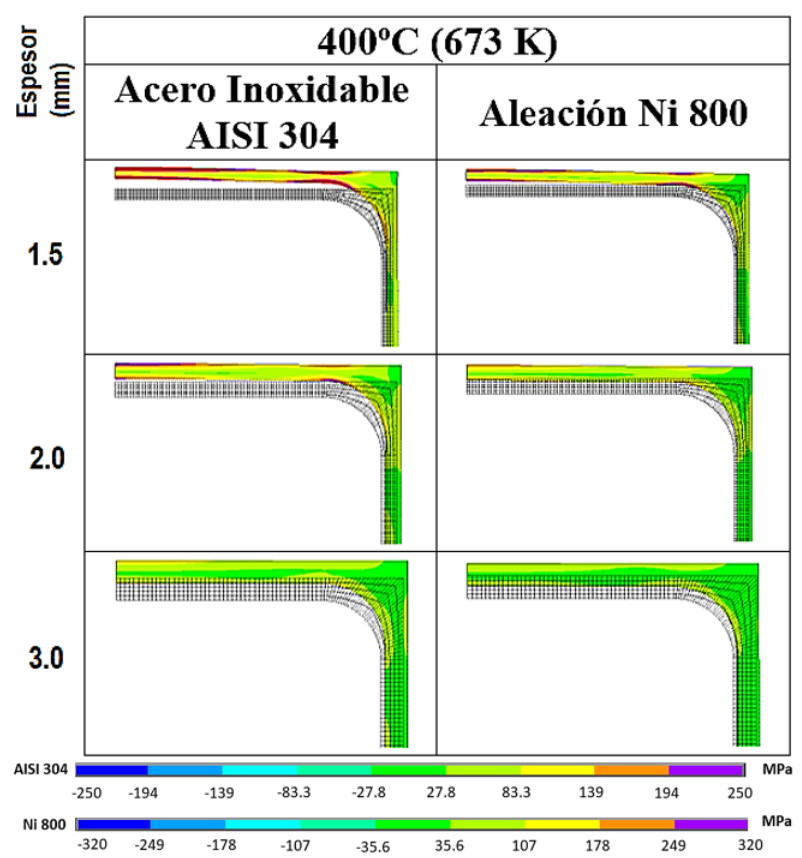

Presión constante $=250$ psi, temperaturas de $400{ }^{\circ} \mathrm{C}$

Se puede verificar que para ambos materiales las tensiones equivalentes máximas tienen una variación mínima con el aumento de temperatura 160, 200 y $400{ }^{\circ} \mathrm{C}$; por lo tanto, en este modelo los resultados solo están influenciados por el cambio en la presión interna. Se presume debido a dos factores: el gradiente de temperatura es mínimo en la pared del reactor debido a la condición estable que se alcanza con el fluido térmico interno (biodiésel) y el flujo convectivo externo (fuego). Por otro lado, ninguna de las temperaturas alcanzadas excede las temperaturas de servicio de los materiales, $850{ }^{\circ} \mathrm{C}$ en el caso del acero inoxidable AISI 304 y $816{ }^{\circ} \mathrm{C}$ en la aleación de níquel Ni800. Por lo tanto, es importante desarrollar un análisis de fatiga en una próxima investigación para estimar la vida útil del reactor.

\subsection{Pruebas de funcionamiento}

Con el prototipo desarrollado según el apartado 2.3 y las consideraciones del análisis numérico (FEM), se procedió a la realización de las pruebas de funcionamiento en laboratorio.

Una vez ejecutado el procedimiento establecido en la metodología, se obtuvo un volumen de biodiésel refinado de color amarillo transparente. Se analizaron las densidades de biodiésel (menor densidad es mejor) obtenidas tomando como referencia temperaturas de $160{ }^{\circ} \mathrm{C}, 180{ }^{\circ} \mathrm{C}$ y $200{ }^{\circ} \mathrm{C}$ utilizadas para la transesterificación (Figura 4). En la comparación de las medias de los resultados del biodiésel se encontraron diferencias estadísticamente significativas; la mayor densidad se 
dio a $200{ }^{\circ} \mathrm{C}(0,89 \mathrm{~g} / \mathrm{ml})$, mientras que a $160{ }^{\circ} \mathrm{C} \mathrm{y}$ $180{ }^{\circ} \mathrm{C}$ la densidad fue de $0,88 \mathrm{~g} / \mathrm{ml}$. El tiempo de obtención del biodiésel comienza de 5 a 10 minutos, siendo estable para obtenerlo después de 8 minutos.

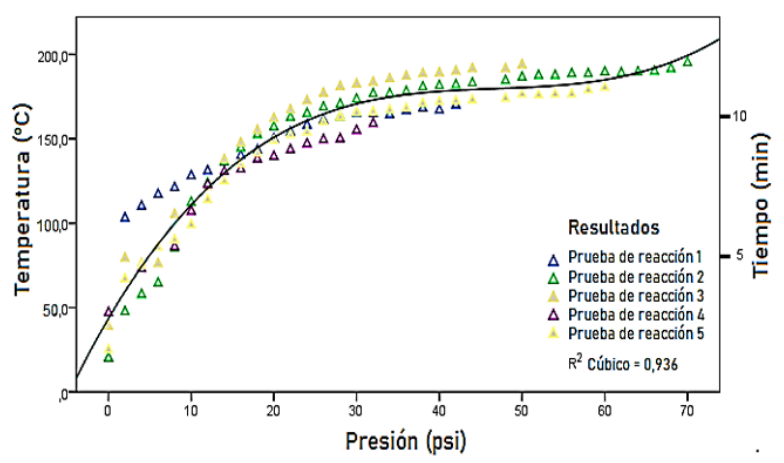

Figura 4. Comportamiento de la reacción interna del reactor en función de la temperatura, la presión y el tiempo

\section{Conclusiones}

El método gráfico de selección de material permite reducir el tiempo de selección del material. La gran cantidad de opciones para la fabricación de un reactor hace imprescindible la necesidad de un refinamiento en la selección al determinar las condiciones de servicio, tales como gradientes de temperatura, rangos de presión, ataques químicos, $\mathrm{pH}$ y consideraciones como el costo y la disponibilidad del material.

Se realizó un análisis multiparamétrico de elementos finitos simétricos en eje 2D utilizando un software Ansys APDL para evaluar la resistencia mecánica del reactor. $\mathrm{Su}$ análisis nos permite determinar la influencia de las variables en el comportamiento termomecánico del reactor. Los resultados muestran que el diseño solo es seguro hasta condiciones subcríticas de presión de 250 psi y un espesor mínimo de $2 \mathrm{~mm}$ en el caso del acero inoxidable AISI 304 donde un factor de seguridad de 1.00. En el caso de la aleación de níquel Ni800, la presión interna se puede aumentar hasta 700 psi y un espesor mínimo de $3 \mathrm{~mm}$ donde el factor de seguridad es 1.10. En las condiciones límite de este modelo, la temperatura no tiene influencia en el estrés equivalente de Von Misses.

En función del diámetro y la longitud de la sección tubular, se obtuvo un volumen de $38,48 \mathrm{~cm}^{3}$, que representa el 3,47\% del volumen total del reactor, por lo que se consideró insignificante. Por otro lado, el flujo de recirculación durante la operación del reactor manejado por la bomba es de $3 \mathrm{l} / \mathrm{min}$; en tal virtud, el fluido que pasa a través de la tubería tiene un tiempo de residencia (sección cilíndrica / flujo de la bomba), igual a $0,026 \mathrm{~min}$ (1,6 segundos), siendo evidente el efecto del comportamiento del flujo del pistón en la sección tubular, no es significativo con respecto al proceso de reacción que ocurrió en el tanque del reactor.
A través del proceso de reacción, se observó que, a mayor temperatura, más jabón se forma, lo que aumenta la densidad del biodiésel. Sin embargo, estos valores relacionados con la densidad del biodiésel obtenido en condiciones subcríticas están dentro del estándar europeo que establece un rango mínimo de $0,86 \mathrm{~g} / \mathrm{ml}$ y un máximo de $0,90 \mathrm{~g} / \mathrm{ml}$. Esta calidad de biodiésel se logró en un tiempo aproximado de 5-8 minutos.

\section{Referencias}

[1] Y. Ma and Y. Liu, "Chapter 21 - biodiesel production: Status and perspectives," in Biofuels: Alternative Feedstocks and Conversion Processes for the Production of Liquid and Gaseous Biofuels (Second Edition), second edition ed., ser. Biomass, Biofuels, Biochemicals, A. Pandey, C. Larroche, C.-G. Dussap, E. Gnansounou, S. K. Khanal, and S. Ricke, Eds. Academic Press, 2019, pp. 503-522. [Online]. Available: https: //doi.org/10.1016/B978-0-12-816856-1.00021-X

[2] L. Faba, E. Díaz, and S. Ordóñez, "Recent developments on the catalytic technologies for the transformation of biomass into biofuels: A patent survey," Renewable and Sustainable Energy Reviews, vol. 51, pp. 273-287, 2015. [Online]. Available: https://doi.org/10.1016/j.rser.2015.06.020

[3] J. Xu, G. Xiao, Y. Zhou, and J. Jiang, "Production of biofuels from high-acid-value waste oils," Energy \& Fuels, vol. 25, no. 10, pp. 4638-4642, 2011. [Online]. Available: https://doi.org/10.1021/ef2006723

[4] M. Mohammadi, G. D. Najafpour, H. Younesi, P. Lahijani, M. H. Uzir, and A. R. Mohamed, "Bioconversion of synthesis gas to second generation biofuels: A review," Renewable and Sustainable Energy Reviews, vol. 15, no. 9, pp. 4255-4273, 2011. [Online]. Available: https://doi.org/10.1016/j.rser.2011.07.124

[5] F. A. Avellaneda Vargas, "Producción y caracterización de biodiésel de palma y de aceite reclicado mediante un proceso batch y un proceso continuo con un reactor helicoidal," 2010. [Online]. Available: https://bit.ly/3jqIgdX

[6] Y. Wang, P. L. Shiyi Ou, and Z. Zhang, "Preparation of biodiesel from waste cooking oil via two-step catalyzed process," Energy Conversion and Management, vol. 48, no. 1, pp. 184-188, 2007. [Online]. Available: http://www.sciencedirect. com/science/article/pii/S0196890406001543

[7] Y. Zhang, M. A. Dubé, D. D. McLean, and M. Kates, "Biodiesel production from waste 
cooking oil: 2. economic assessment and sensitivity analysis," Bioresource Technology, vol. 90, no. 3, pp. 229-240, 2003. [Online]. Available: https://doi.org/10.1016/S0960-8524(03)00150-0

[8] S. Zheng, M. Kates, M. Dubé, and D. McLean, "Acid-catalyzed production of biodiesel from waste frying oil," Biomass and Bioenergy, vol. 30, no. 3, pp. 267-272, 2006. [Online]. Available: https://doi.org/10.1016/j.biombioe.2005.10.004

[9] A. Srivastava and R. Prasad, "Triglyceridesbased diesel fuels," Renewable and Sustainable Energy Reviews, vol. 4, no. 2, pp. 111-133, 2000. [Online]. Available: https://doi.org/10.1016/S1364-0321(99)00013-1

[10] B. H. Pedro, A. S. John, and G. Cano, "Estudio experimental de las variables que afectan la reacción de transesterificación del aceite crudo de palma para la producción de biodiesel," Scientia et Technica, vol. 1, no. 24, ene. 2004. [Online]. Available: https://doi.org/10.22517/23447214.7323

[11] B. R. Moser, Biodiesel Production, Properties, and Feedstocks. New York, NY: Springer New York, 2011, pp. 285-347. [Online]. Available: https://doi.org/10.1007/978-1-4419-7145-6_15

[12] A. da Silva César, D. E. Werderits, G. L. de Oliveira Saraiva, and R. C. da Silva Guabiroba, "The potential of waste cooking oil as supply for the brazilian biodiesel chain," Renewable and Sustainable Energy Reviews, vol. 72, pp. 246-253, 2017. [Online]. Available: https://doi.org/10.1016/j.rser.2016.11.240

[13] A. Villabona Ortiz, R. Iriarte Pico, and C. Tejada Tovar, "Alternativas para el aprovechamiento integral de residuos grasos de procesos de fritura," Teknos revista científica, vol. 17, no. 1, pp. 21-29, jul. 2017. [Online]. Available: https://doi.org/10.25044/25392190.890

[14] Gobierno de España. (2020) Ministerio de Transición Ecológica y el Reto Demográfico. [Online]. Available: https://bit.ly/37zY23X

[15] W. D. Callister Jr. and D. G. Rethwisch, Materials science and engineering: an introduction. John Wiley \& Sons, Inc., 2018. [Online]. Available: https://bit.ly/3oo2Krl

[16] H. A. González and D. H. Mesa, "La importancia del método en la selección de materiales," Scientia
Et Technica, vol. X, pp. 175-180, 2004. [Online]. Available: https://bit.ly/37EHrMs

[17] R. V. Rao, "A material selection model using graph theory and matrix approach," Materials Science and Engineering: A, vol. 431, no. 1, pp. 248-255, 2006. [Online]. Available: https://doi.org/10.1016/j.msea.2006.06.006

[18] M. F. Ashby and K. Johnson, Materials Selection in Mechanical Design. Elsevier Ltd., 2016. [Online]. Available: https: //doi.org/10.1016/C2009-0-25539-5

[19] M. F. Ashby, H. Shercliff, and D. Cebon, Materials: engineering, science, processing and design. Butterworth-Heinemann, 2018. [Online]. Available: https://bit.ly/3dSpRW4

[20] T. E. Tezduyar and Y. J. Park, "Discontinuitycapturing finite element formulations for nonlinear convection-diffusion-reaction equations," Computer Methods in Applied Mechanics and Engineering, vol. 59, no. 3, pp. 307-325, 1986. [Online]. Available: https://doi.org/10.1016/0045-7825(86)90003-4

[21] W. F. Ramírez, Computational methods for process simulation. Butterworth-Heinemann, 1997. [Online]. Available: https://bit.ly/3mhsfc3

[22] A. Chakrabarty, S. Mannan, and T. Cagin, Multiscale Modeling for Process Safety Applications. Butterworth-Heinemann, 2015. [Online]. Available: https://bit.ly/2HujBbc

[23] W. B. J. Zimmerman, Process modelling and simulation with finite element methods. World Scientific, 2004. [Online]. Available: https://bit.ly/2G0a9vM

[24] R. Woodbury, Elements of parametric design. Routledge, 2010. [Online]. Available: https://bit.ly/2Hu2c2s

[25] R. de Luca, P. Fanelli, S. Mingozzi, G. Calabró, F. Vivio, F. Maviglia, and J. You, "Parametric design study of a substrate material for a demo sacrificial limiter," Fusion Engineering and Design, vol. 158, p. 111721, 2020. [Online]. Available: https://doi.org/10.1016/j.fusengdes.2020.111721

[26] J. Monedero, "Parametric design: a review and some experiences," Automation in Construction, vol. 9, no. 4, pp. 369-377, 2000. [Online]. Available: https://doi.org/10.1016/S0926-5805(99)00020-5 


\title{
Algoritmo DE PREDICCIÓN DEL CONSUMO DE COMBUSTIBLE PARA MEZCLA DE ETANOL ANHÍDRIDO EN CIUDADES DE ALTURA PREDICTION ALGORITHM OF FUEL CONSUMPTION FOR ANHYDROUS ETHANOL MIXTURE IN HIGH-ALTITUDE CITIES
}

\author{
Fabricio Espinoza $^{1}$, Fredy Tacuri ${ }^{1}$, Wilmer Contreras ${ }^{1, *}$, Javier Vázquez $^{1}$ \\ Recibido: 15-05-2020, Revisado: 05-08-2020, Aprobado tras revisión: 30-09-2020
}

\section{Resumen}

En la presente investigación se obtiene un modelo matemático de predicción del consumo específico de combustible en un motor ciclo Otto de 1,4 litros con inyección electrónica sin hacer modificaciones, al usar como combustible mezclas de gasolina con concentraciones a partir de $0 \%, 25 \%, 50 \%, 75 \%$ y $100 \%$ en volumen de etanol anhidro. Para el análisis de los resultados se realizó un diseño de experimento de mezcla reticular simplex lattice, el cual se sometió a un ciclo de conducción urbano de la ciudad de Cuenca a $2558 \mathrm{~m} \mathrm{s.} \mathrm{n.} \mathrm{m.,} \mathrm{en} \mathrm{un} \mathrm{banco} \mathrm{de} \mathrm{potencia} \mathrm{de} \mathrm{rodillos.}$ La adquisición de datos y la obtención del algoritmo fueron a través de un análisis de métodos estadísticos descriptivos. La validación del algoritmo se realizó por medio del análisis de residuos. Como resultado principal se cuenta con un modelo matemático, el que permite predecir el consumo de combustible del motor, para rangos de concentración de etanol del $0 \%$ al $100 \%$ en la gasolina sin la necesidad de realizar pruebas reales.

Palabras clave: etanol anhídrido, modelo explicativo, consumo combustible

\section{Abstract}

In the present research work, a mathematical model is obtained for predicting specific fuel consumption in a 1.4-liter Otto cycle engine with electronic injection without making modifications, when using as fuel gasoline mixtures with concentrations in volume of $0 \%, 25 \%, 50 \%, 75 \%$ and $100 \%$ of anhydrous ethanol. For the analysis of results, a simplex lattice reticular mixture experiment design was carried out, which was subject to an urban driving cycle in the city of Cuenca at $2558 \mathrm{~m}$ above sea level in a roller power bank. The data acquisition and the development of the algorithm were carried out through an analysis of descriptive statistical methods. The validation of the algorithm was performed through residual analysis. As a main result, there is a mathematical model that enables predicting the engine fuel consumption, for ranges of ethanol concentration from $0 \%$ to $100 \%$ in the gasoline without needing to conduct real tests.

Keywords: Ethanol anhydride; explanatory model; fuel consumption

\footnotetext{
1,* Universidad Politécnica Salesiana, Cuenca-Ecuador. Autor para correspondencia ${ }^{-}$rcontreras@ups.edu.ec.

(D) http://orcid.org/0000-0003-4559-4474 (i) http://orcid.org/0000-0002-4160-2898

(D) http://orcid.org/0000-0003-2300-9457 (D) http://orcid.org/0000-0001-9678-5364
}

Forma sugerida de citación: Espinoza, F.; Tacuri, F.; Contreras, W. y Vásquez, J. (2021). «Algoritmo de predicción del consumo de combustible para mezcla de etanol anhídrido en ciudades de altura». InGENIUS. N. ${ }^{\circ} 25$, (enero-junio). pp. 41-49. DOI: https://doi.org/10.17163/ings.n25.2021.04. 


\section{Introducción}

La preocupación por la contaminación ambiental debido a los residuos de la combustión incompleta y el agotamiento de los combustibles de origen fósil motiva al estudio sobre la reformulación de mezclas con combustibles alternativos. Una opción viable es aplicar combinaciones de etanol y gasolina, la cual puede reducir la contaminación del aire y al mismo tiempo ofrecer una mejora en el rendimiento del motor en comparación con el combustible de petróleo sin mezclar.

$\mathrm{Al}$ evaluar el efecto de estas mezclas, se tiene una variación en el consumo total de combustible, en esta línea de estudio, según el trabajo de Al-Hasan, (2003) [1]. En un vehículo Toyota Tercel de cuatro cilindros y encendido por chispa de cuatro tiempos con una cilindrada de 1,4 litros, una relación de compresión de 9:1 y una potencia máxima de $52 \mathrm{~kW}$ a $5600 \mathrm{rpm}$, da como resultado el aumento del consumo de combustible en aproximadamente $8,3 \%, 9,0 \%, 7$ $\%$ y $5,7 \%$.

Cuando se utilizan biocombustibles en motores de combustión interna, el consumo de estos aumenta [2]. Esto se debe a que si se disminuye la relación estequiométrica de aire-combustible para las mismas revoluciones por minuto, el mismo nivel de carga y la misma masa de aire, la masa de combustible que se necesita debe ser mayor [3].

Fernández, Mosquera y Mosquera [4] demuestran que la utilización de etanol mezclado con la gasolina aumenta el consumo linealmente a la mezcla utilizada.

La investigación realizada en un vehículo Lada 1.3 litros de cuatro tiempos, con un sistema de alimentación por carburación, el combustible utilizado es una mezcla de etanol anhidro en gasolina regular, 10, 20 y $30 \%$, de la cual Melo y colaboradores [5] concluyen que a medida que aumenta el porcentaje de etanol en la mezcla con gasolina, el consumo de combustible aumenta para todos los puntos experimentales evaluados.

El estudio realizado en un vehículo marca Ford de 1,4 litros respecto al consumo de combustible para mezclas de etanol y gasolina según Delión y Rojas [6], manifiesta que el «incremento del consumo de combustible es mayor ante el aumento de carga o par motor, conservando los rpm constantes y las emisiones contaminantes son menores que con gasolina pura».

Por su parte, estudios realizados en 1997, 1998 y 1999 por Kortum et al. [7], Apace [8] y Ragazzi y Nelson [9], respectivamente, coinciden con las investigaciones del 2003 por Al-Hassan [1], He et al. [10] y Patzek [11], del 2004 por Wu et al. [12], en 2005 por Coelho et al. [13], Hansen et al., Niven [14] y American Coalition for Ethanol [15], del 2006 por Behrentz [16], Durbin et al. [17], Shapiro [18] y Yucesu et al. [19], del 2008 por Acevedo et al. [20], como de los trabajos más recientes del 2013 por Doe et al. [21], en que el con- sumo de combustible aumenta del 1 al $6 \%$ en motores sin modificar con mezclas de 0-25\% de etanol, ya que el consumo depende del sistema de control electrónico del motor.

Las investigaciones desarrolladas sobre el consumo de combustible para mezclas etanol anhidro y gasolina concluyen que aumenta según se incrementa el grado de concentración de etanol anhidro en la gasolina.

Estas investigaciones dentro de su metodología no consideran el desarrollo de un diseño experimental por mezclas en conjunto a la aplicación de un ciclo de conducción propio para las ciudades de altura.

Este trabajo se desarrolla con el objetivo de obtener un algoritmo matemático que permita calcular el consumo específico de combustible en un vehículo Hyundai Getz 1,4 litros para diferentes mezclas etanol anhidro y gasolina en condición de una altura alrededor de $2558 \mathrm{~m} \mathrm{s.} \mathrm{n.} \mathrm{m}$.

\section{Materiales y métodos}

La metodología aplicada consiste en un diseño experimental por mezclas simplex reticular (q, m), que considera $q$ componentes y permite ajustar un modelo estadístico de orden (m); lo cual consiste en todas las posibles combinaciones de componentes o mezclas que se puedan formar al considerar que las proporciones puedan tomar los $(m+1)$ valores entre cero y uno, dados por la Ecuación 1 [22].

$$
\boldsymbol{x i}=\frac{0.1}{m}, \frac{0.2}{m}, \ldots, \frac{m}{m}
$$

La mezcla etanol anhidro-gasolina, se identifica con la nomenclatura (E) seguido de un número, la letra representa la mezcla y el número indica el porcentaje de etanol que se adiciona a la gasolina. Esta mezcla es caracterizada por su densidad la que se obtiene por el método del picnómetro; el octanaje mediante un octanómetro el que cumple con la norma ASTM 2699 - 86 y el poder calorífico superior e inferior $\left(\boldsymbol{H}_{c s} E x\right),\left(\boldsymbol{H}_{c i} E x\right)$ según las Ecuaciones 2 y 3 , que permite calcular esta propiedad [23].

$$
\begin{aligned}
& \boldsymbol{H}_{c s E x}=\% \boldsymbol{E} \times \boldsymbol{H}_{C s \text { etanol }}+\% G \times \boldsymbol{H}_{C s \text { gasolina }} \\
& \boldsymbol{H}_{c i \text { Ex }}=\% \boldsymbol{E} \times \boldsymbol{H}_{C i \text { etanol }}+\% G \times \boldsymbol{H}_{C i \text { gasolina }}
\end{aligned}
$$

Para obtener el modelo matemático los datos son validados estadísticamente mediante la prueba de valores atípicos, se ajusta a un modelo estadístico para investigar el efecto de los componentes sobre la respuesta. Una primera aproximación puede ser ajustada en un modelo de primer orden (Ecuación 4).

$$
E(y)=\sum_{i=1}^{q-1} \beta_{i} \chi_{i}
$$


Cuando se ajusta un modelo cuadrático también es necesario incorporar la restricción, $x 1+x 2+\ldots+x q=1$; ya que esto le dará una característica especial al modelo. Para ilustrar la idea se supone que, se tienen tres componentes, $x 1, x 2, x 3$ por lo que el polinomio de segundo grado está dado por la Ecuación 5.

$$
E(y)=\sum_{i=1}^{q} \beta_{i} \chi_{i}+\sum_{i<j} \sum_{j=2}^{q} \beta_{i j} \chi_{i} \chi_{j}
$$

Si el modelo cuadrático no es suficiente para describir la respuesta, se puede recurrir al modelo cúbico especial (Ecuación 6).

$$
\begin{array}{r}
E(y)=\sum_{i=1}^{q} \beta_{i} \chi_{i}+\sum_{i<j} \sum_{j=2}^{q} \beta_{i j} \chi_{i} \chi_{j}+ \\
+\sum_{i<j} \sum_{j=2}^{q} \delta_{i j} \chi_{i} \chi_{j}\left(\chi_{i}-\chi_{j}\right)+ \\
+\sum_{i<j} \sum_{j<k} \sum_{j=3}^{f q} \beta_{i j k} \chi_{i} \chi_{j} \chi_{k}
\end{array}
$$

Para la obtención del algoritmo matemático de consumo de combustible se establece la metodología indicada en la Figura 1.

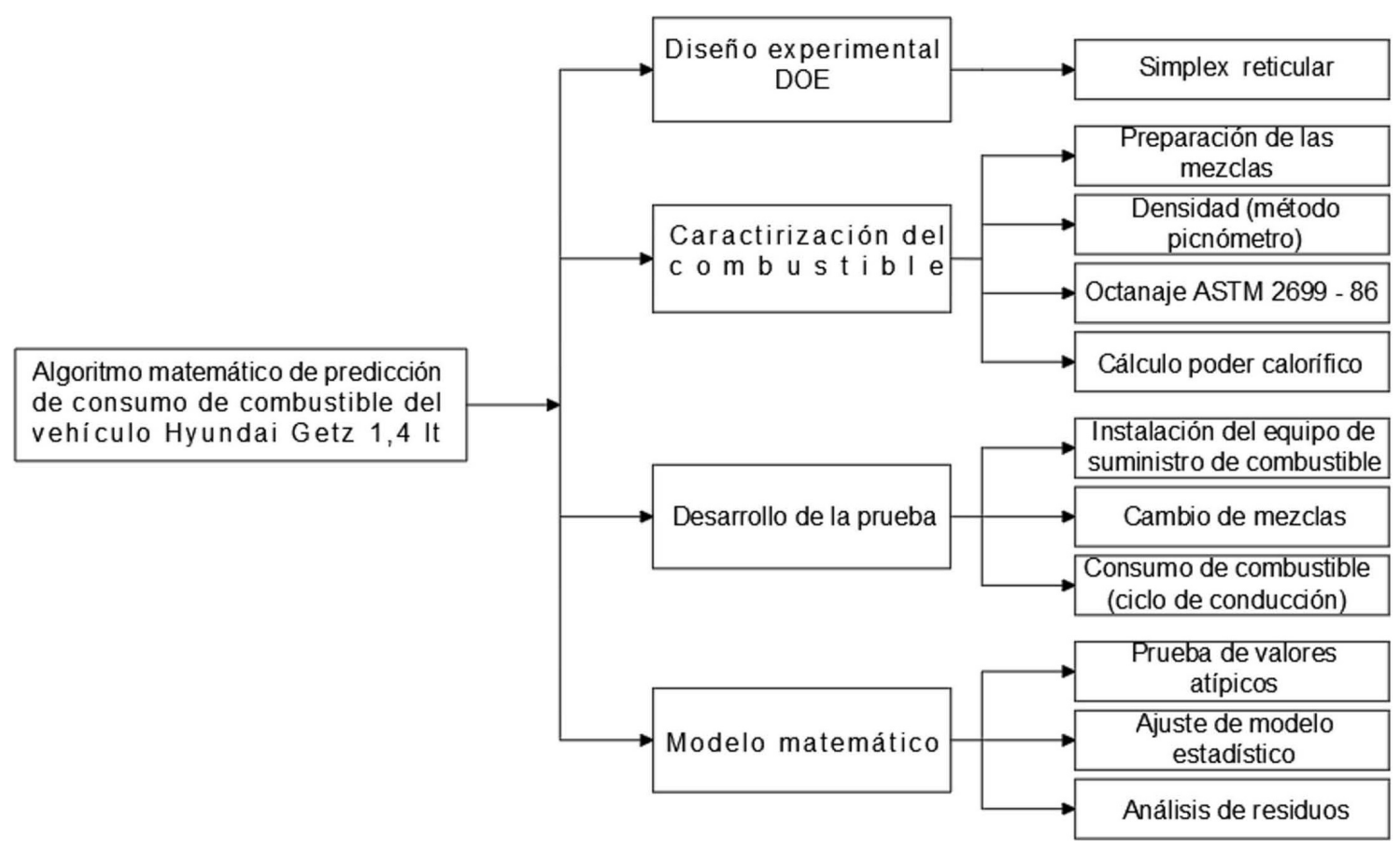

Figura 1. Metodología aplicada 1

\subsection{Diseño de experimental}

El diseño experimental utilizado es reticular simplex lattice de mezclas, mediante el software Minitab 17 Statistical, ver Tabla 1, en la que se indica el número de componentes que para el caso son el etanol anhidro y la gasolina, puntos de diseño, 21; grado del retículo utilizado, 4; además, el orden de corridas experimentales es aleatorizado.

\subsection{Caracterización de las mezclas}

El combustible utilizado es una mezcla de etanol anhidro con gasolina, los que cumplen las especificaciones de las normas NTE INEN 2 478:2009 [24] y NTE INEN 935:2010 [25], respectivamente. Las mezclas utilizadas son caracterizadas, ver Tabla 2, en la que se indica la densidad, el octanaje y el poder calorífico. 
Tabla 1. Diseño experimental aleatorizado

\begin{tabular}{cccccc}
\hline Orden establecido & Orden corrida & Tipo Pt. & Bloques & ETANOL & GASOLINA \\
\hline $\mathbf{1 7}$ & 1 & 0 & 1 & 0,5 & 0,5 \\
$\mathbf{1 6}$ & 2 & 2 & 1 & 0,25 & 0,75 \\
$\mathbf{3}$ & 3 & 0 & 1 & 0,5 & 0,5 \\
$\mathbf{1 4}$ & 4 & -1 & 1 & 0,75 & 0,25 \\
$\mathbf{9}$ & 5 & 2 & 1 & 0,25 & 0,75 \\
$\mathbf{1 9}$ & 6 & 1 & 1 & 1 & 0 \\
$\mathbf{2}$ & 7 & 2 & 1 & 0,25 & 0,75 \\
$\mathbf{1 3}$ & 8 & -1 & 1 & 0,25 & 0,75 \\
$\mathbf{1 0}$ & 9 & 0 & 1 & 0,5 & 0,5 \\
$\mathbf{1}$ & 10 & 1 & 1 & 0 & 1 \\
$\mathbf{1 2}$ & 11 & 1 & 1 & 1 & 0 \\
$\mathbf{2 1}$ & 12 & -1 & 1 & 0,75 & 0,25 \\
$\mathbf{7}$ & 13 & -1 & 1 & 0,75 & 0,25 \\
$\mathbf{1 5}$ & 14 & 1 & 1 & 0 & 1 \\
$\mathbf{1 1}$ & 15 & 2 & 1 & 0,75 & 0,25 \\
$\mathbf{1 8}$ & 16 & 2 & 1 & 0,75 & 0,25 \\
$\mathbf{2 0}$ & 17 & -1 & 1 & 0,25 & 0,75 \\
$\mathbf{8}$ & 18 & 1 & 1 & 0 & 1 \\
$\mathbf{5}$ & 19 & 1 & 1 & 1 & 0 \\
$\mathbf{6}$ & 20 & -1 & 1 & 0,25 & 0,75 \\
$\mathbf{4}$ & 21 & 2 & 1 & 0,75 & 0,25 \\
\hline
\end{tabular}

Tabla 2. Propiedades fisicoquímicas de las mezclas

\begin{tabular}{ccccc}
\hline \multirow{2}{*}{ Combustible } & \multicolumn{4}{c}{ Propiedades fisicoquímicas } \\
\cline { 2 - 5 } & Densidad $\mathbf{( k g / \mathbf { m } ^ { 3 } )}$ & $\begin{array}{c}\text { Octanaje } \\
\text { (RON) }\end{array}$ & $\begin{array}{c}\text { Poder calorífico } \\
\text { superior } \mathbf{( k J} / \mathbf{k g})\end{array}$ & $\begin{array}{c}\text { Poder calorífico } \\
\text { inferior } \mathbf{( k J} / \mathbf{k g})\end{array}$ \\
\hline E0 & 740 & 85,6 & 47300 & 44000 \\
E25 & 760 & 90,95 & 42900 & 39725 \\
E50 & 768,7 & 96,3 & 38500 & 35450 \\
E75 & 782 & 101,65 & 34100 & 31175 \\
E100 & 790,7 & 107 & 29700 & 26900 \\
\hline
\end{tabular}

\subsection{Medición de consumo de combustible}

Esta investigación se desarrolla en la ciudad de Cuenca (Ecuador) ubicada a $2558 \mathrm{~m} \mathrm{s.} \mathrm{n.} \mathrm{m.,} \mathrm{las} \mathrm{pruebas} \mathrm{de}$ consumo de combustible se realizan en un vehículo marca Hyundai Getz 1,4 litros con sistema de inyección electrónico y tratamiento de gases por convertidor catalítico de tres vías; la relación de compresión es de 9,5:1, sistema de distribución DOHC con cuatro válvulas por cilindro y de aspiración tipo atmosférico Hyundai Motor Company (2011) [26].

Se prescinde del sistema de alimentación de combustible original del vehículo y se instala un equipo alterno de suministro cumpliendo las especificaciones técnicas del fabricante, para el desarrollo de las diferentes pruebas de esta investigación, todo esto con la finalidad de evitar alteraciones de las mezclas de combustible.

El equipo utilizado para la medición de consumo de combustible es flujómetro FLOW-MASTER MAHA
CH-4123. La Figura 2 indica la instalación del equipo en el vehículo; los datos obtenidos se indican en la Tabla 3 de acuerdo con el diseño de experimento.

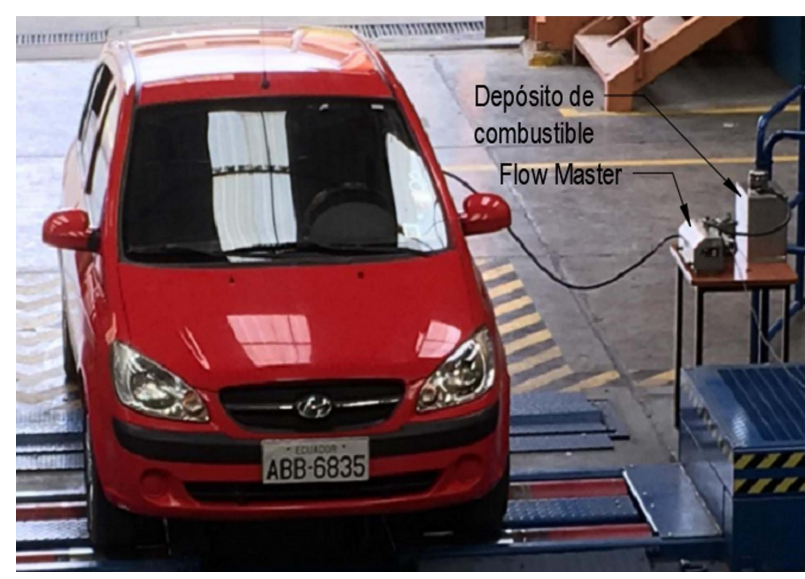

Figura 2. Pruebas de funcionamiento 1 
Tabla 3. Medición de consumo de combustible

\begin{tabular}{ccccc}
\hline Orden establecido & Orden corrida & ETANOL & GASOLINA & $\begin{array}{c}\text { CONSUMO DE } \\
\text { COMBUSTIBLE (g/km) }\end{array}$ \\
\hline $\mathbf{1 7}$ & 1 & 0,5 & 0,5 & 0,0435 \\
$\mathbf{1 6}$ & 2 & 0,25 & 0,75 & 0,0405 \\
$\mathbf{3}$ & 3 & 0,5 & 0,5 & 0,0405 \\
$\mathbf{1 4}$ & 4 & 0,75 & 0,25 & 0,037 \\
$\mathbf{9}$ & 5 & 0,25 & 0,75 & 0,0475 \\
$\mathbf{1 9}$ & 6 & 1 & 0 & 0,0475 \\
$\mathbf{2}$ & 7 & 0,25 & 0,75 & 0,0495 \\
$\mathbf{1 3}$ & 8 & 0,25 & 0,75 & 0,05 \\
$\mathbf{1 0}$ & 9 & 0,5 & 0,5 & 0,04 \\
$\mathbf{1}$ & 10 & 0 & 1 & 0,043 \\
$\mathbf{1 2}$ & 11 & 1 & 0 & 0,0515 \\
$\mathbf{2 1}$ & 12 & 0,75 & 0,25 & 0,045 \\
$\mathbf{7}$ & 13 & 0,75 & 0,25 & 0,0465 \\
$\mathbf{1 5}$ & 14 & 0 & 1 & 0,036 \\
$\mathbf{1 1}$ & 15 & 0,75 & 0,25 & 0,0475 \\
$\mathbf{1 8}$ & 0,75 & 0,25 & 0,047 \\
$\mathbf{2 0}$ & 16 & 0,25 & 0,04 \\
$\mathbf{8}$ & 17 & 0 & 1 & 0,036 \\
$\mathbf{5}$ & 18 & 1 & 0 & 0,0515 \\
$\mathbf{6}$ & 19 & 0,25 & 0,75 & 0,0355 \\
$\mathbf{4}$ & 20 & 0,75 & 0,25 & 0,049 \\
\hline & 21 & & & \\
\hline
\end{tabular}

Para obtener el consumo de combustible específico se utilizó el ciclo de conducción representativo para la ciudad de Cuenca, el cual se identifica en la Figura 3, se aplica un microciclo con los cinco primeros minutos ya que mediante un análisis preexperimental son los más distintivos. El ensayo se realiza sobre un banco de potencia Maha LPS 3000.

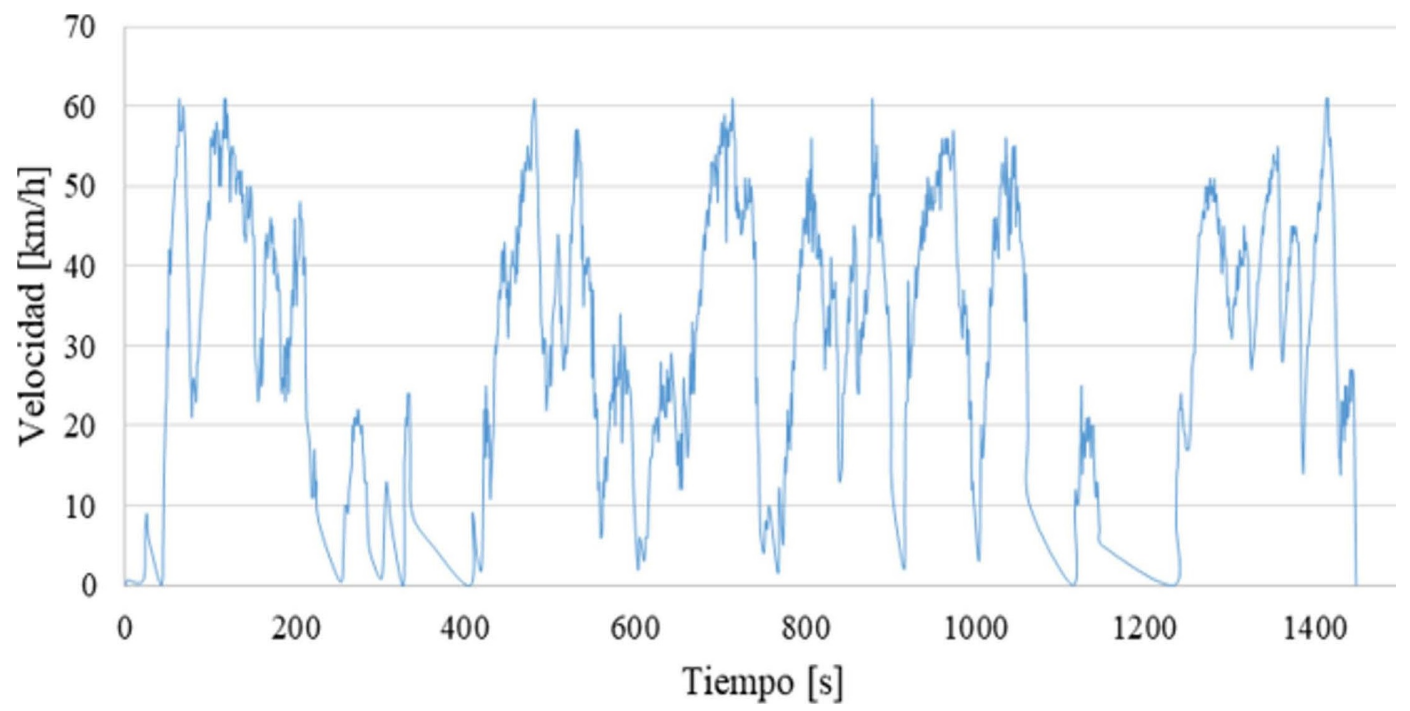

Figura 3. Ciclo de conducción representativo de la ciudad de Cuenca

\subsection{Tratamiento de los datos para el modelo de respuesta}

Con los datos del diseño experimental en función de las diferentes mezclas, conviene efectuar pruebas de validación de los resultados antes de proceder a obtener el modelo de respuesta. Para la validación de los resultados se establece la prueba de valores atípicos (outlier) de R22 de Dixon [27]. Validados los datos se construye el modelo y se realiza el análisis de residuos con la finalidad de comprobar la hipótesis de normalidad, homocedasticidad, independencia y linealidad del modelo. 


\section{Resultados y discusión}

Para determinar el modelo explicativo del consumo de combustible, se realiza una regresión lineal multivariable con los datos obtenidos del DOE y se ajusta a un orden superior del modelo según se indica en la Tabla 4 , donde se observan los diferentes valores que toma « $\mathrm{R}^{2} » \mathrm{y}$ el «p-valor». Esta información permite seleccionar el modelo lineal, por cumplir con los supuestos, además en la columna de «p-valor» se observa que los demás modelos de grado superior son mayores al nivel de significancia de 0,05 , por lo tanto, a estos modelos no se los considera.

Una vez seleccionado el modelo lineal, se determinan los coeficientes de regresión estimados (Tabla 5), donde se obtiene la fórmula del modelo para la predicción del consumo, visto en la Ecuación 7.

$$
2 Y=0.05100(\text { etanol })+0,03455(\text { gasolina })
$$

Tabla 4. Resumen ajustes de los modelos para consumo combustible

\begin{tabular}{ccccc}
\hline Modelo & P-valor & $\mathbf{( \% )}$ & predicción (\%) & ajustado (\%) \\
\hline Lineal & 0 & 94,2 & 93,04 & 93,86 \\
Cuadrático & 0,274 & 94,6 & 92,66 & 93,94 \\
Cúbico completo & 0,784 & 94,6 & 91,92 & 93,62 \\
Cuártico completo & 0,126 & 95,3 & 91,59 & 94,17 \\
Nota. Valor *p $<\mathbf{0 , 0 5}$ & & & \\
\hline
\end{tabular}

Tabla 5. Coeficientes de regresión estimados para consumo combustible

\begin{tabular}{cccccc}
\hline Término & Coef. & EE del coef. & T & P & VIF \\
\hline ETANOL & 0,051 & 0,000562 & $*$ & $*$ & 1,19 \\
GASOLINA & 0,03455 & 0,000562 & $*$ & $*$ & 1,19 \\
Nota. Valor ${ }^{*} \mathbf{p}<\mathbf{0 , 0 5}$ & & & & \\
\hline
\end{tabular}

Al aplicar la Ecuación 7 se obtienen los resultados indicados en la Figura 4.

Además, en el análisis de varianza para el modelo lineal según se indica en la Tabla 6, el $\ll \mathrm{p}$-valor» $=0,000$, por lo tanto, el modelo es significativo y con un $« \mathrm{R}^{2} »$ ajustado de 93,86 muy bueno.

Los demás modelos se excluyen por no cumplir con el supuesto del p-valor [28].

Una vez realizado el análisis de la varianza es- tandarizado, se presentan a continuación los resultados gráficos que confirman la adecuación del modelo para el consumo de combustible.

Para evaluar el modelo explicativo del consumo de combustible, se utiliza la gráfica de residuos cuatro en uno, según se indica en la Figura 5. Este análisis gráfico, con respecto a los residuos estandarizados, permite la verificación de la adecuación del modelo experimental obtenido anteriormente.

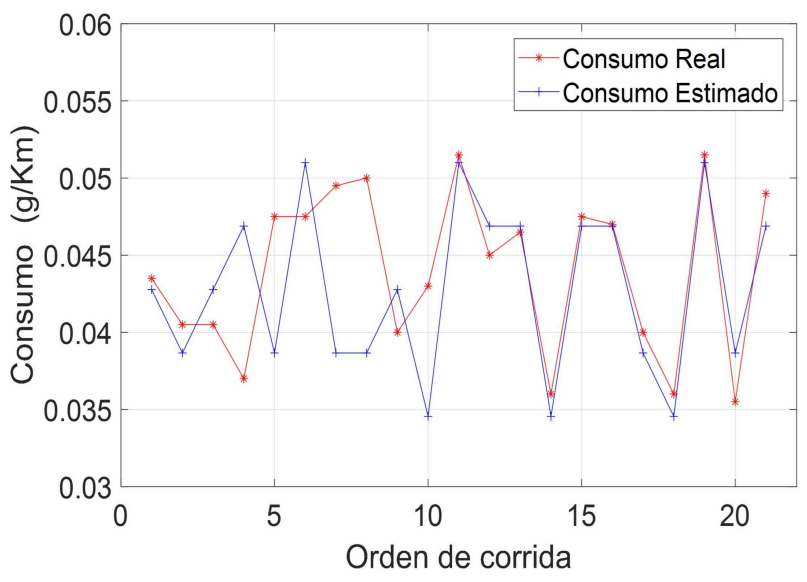

Figura 4. Resultado del algoritmo numérico 
Tabla 6. Análisis de varianza para consumo combustible

\begin{tabular}{ccccccc}
\hline Fuente & GL & SC Sec. & SC Ajust. & MC Ajust. & F & P \\
\hline Regresión & 1 & 0,00061 & 0,000609 & 0,000609 & 306,5 & 0 \\
$\quad$ Lineal & 1 & 0,00061 & 0,000609 & 0,000609 & 306,5 & 0 \\
Error residual & 19 & $3,8 \mathrm{E}-05$ & 0,000038 & 0,000002 & & \\
Falta de ajuste & 3 & $8 \mathrm{E}-06$ & 0,000008 & 0,000003 & 1,34 & 0,3 \\
Error puro & 16 & 0,00003 & 0,00003 & 0,000002 & & \\
$\quad$ Total & 20 & 0,00065 & & & & \\
Nota. Valor $* \mathbf{p}<\mathbf{0 , 0 5}$ & & & & & \\
\hline
\end{tabular}

\section{GRÁFICA DE RESIDUOS PARA CONSUMO COMBUSTIBLE}

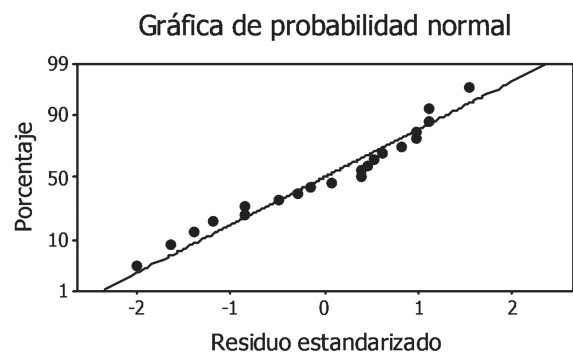

vs. ajustes
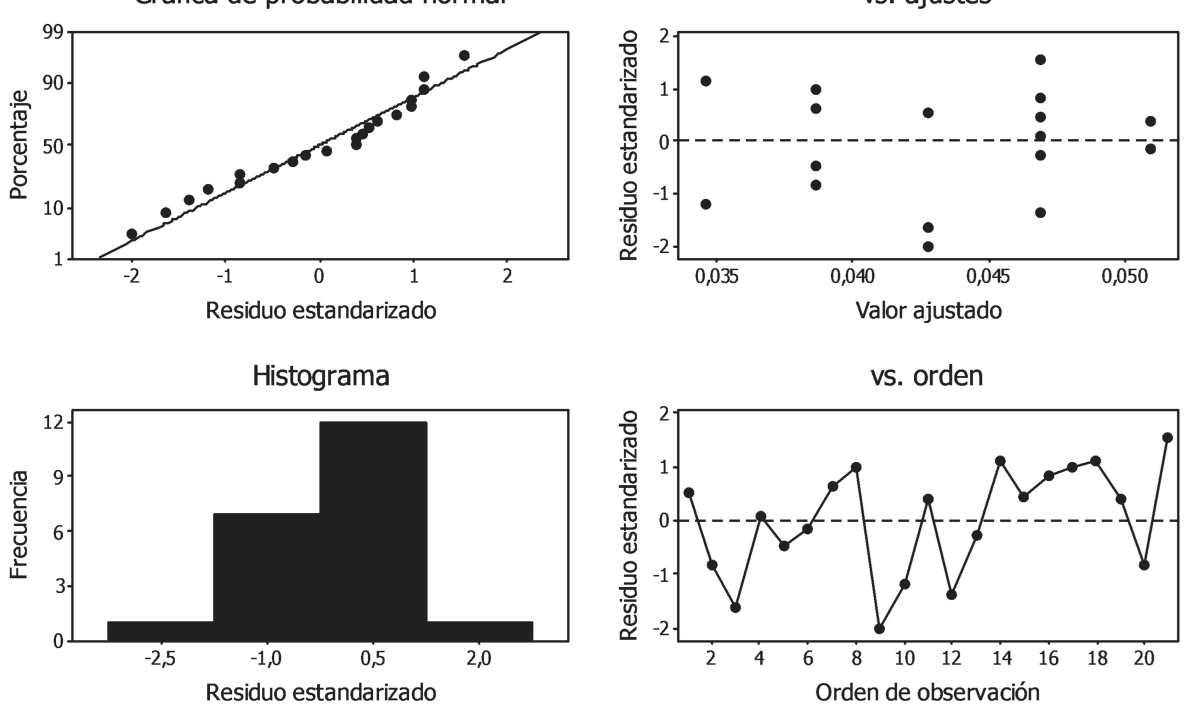

Figura 5. Gráfica de residuales para los datos del consumo combustible

Del análisis de la Figura 5, se tienen las siguientes conclusiones:

a. La gráfica de probabilidad normal muestra que los residuales siguen una distribución normal por ajustarse a la línea de tendencia normal.

b. La gráfica del histograma de residuales sigue la forma de una campana, apreciándose un valor para la observación número nueve de $-2,02$ fuera del rango permitido de $(-2,0)$ en residuo estándar, pero que no tiene mayor significancia en el modelo, por lo tanto, se acepta la normalidad de los datos.

c. Por su parte, en la gráfica de residuos estandarizados versus valores ajustados no se observa una tendencia anormal que indique una mala adecuación del modelo, ya que los residuales se hallan distribuidos aleatoriamente alrededor de cero, por lo tanto, se considera que existe independencia. d. Finalmente, en la gráfica de residuos de estandarizados versus orden de las observaciones, se tiene un patrón aleatorio alrededor de la línea central, no existe una tendencia ascendente o descendente de las observaciones que hablen de una mala adecuación del modelo.

Con este análisis se concluye que, la varianza es correcta y que el modelo no presenta anomalías en los resultados de este y puede ser usado para predecir de mejor manera la variable consumo de combustible.

La gráfica de respuesta de mezcla (etanol-gasolina) en el consumo de combustible, se indica en la Figura 6. Esta permite evaluar cómo se relacionan los componentes con la respuesta utilizando un modelo ajustado.

La gráfica de rastreo de consumo de combustible indicada en la Figura 6, provee la siguiente información sobre los efectos de los componentes:

Se observa que el consumo de combustible tiene una tendencia a aumentar a partir del incremento del etanol hasta llegar a un máximo en E100, mientras decrece la concentración del segundo componente en este caso la gasolina extra. 
Nótese también que la pendiente de la curva es más pronunciada en el tramo comprendido entre 0 a un $15 \%$ de etanol y el otro tramo a partir de $40 \%$, por lo tanto, el rango entre 15 al $40 \%$ es menor pendiente, correspondiendo a la zona en la que el consumo de combustible es menor.

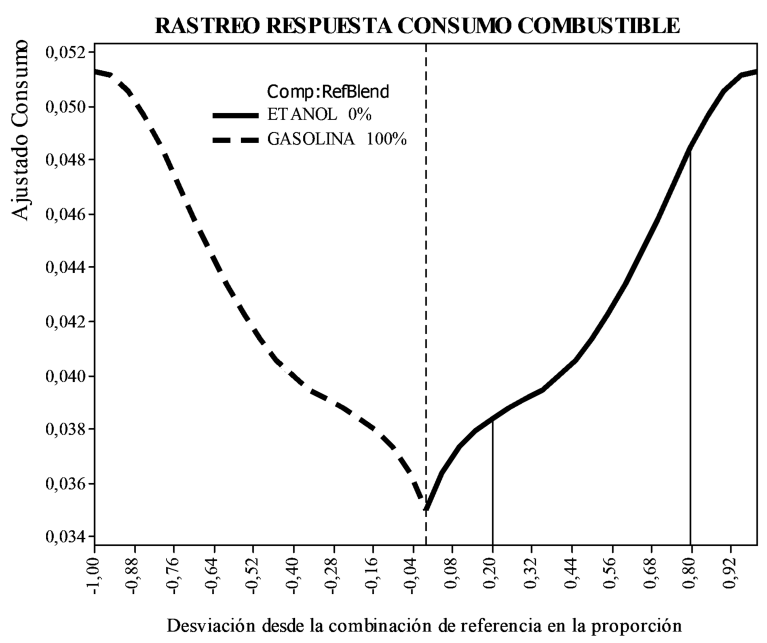

Figura 6. Gráfica de rastreo respuesta consumo de combustible

\section{Conclusiones y recomendaciones}

El consumo específico de combustible es directamente proporcional al porcentaje de etanol en la mezcla, en otras palabras, a mayor porcentaje de etanol en la mezcla mayor será el consumo específico del motor. Así con una mezcla E20 el aumento de consumo represente un $7 \%$ y en una mezcla E 100 es de 31,3\% más con respecto a la gasolina de 86,5 octanos.

Se estableció un modelo matemático que permite determinar el consumo de combustible para diferentes porcentajes de etanol en la gasolina aplicable para el contraste de pruebas reales.

El incremento del consumo de combustible se explica debido a la disminución del poder calorífico de la mezcla según varíe la concentración de etanol.

Durante el desarrollo de las pruebas el vehículo funcionó correctamente sin presentar anomalías para concentraciones de etanol superiores al $30 \%$.

\section{Referencias}

[1] M. Al-Hasan, "Effect of ethanol-unleaded gasoline blends on engine performance and exhaust emission," Energy Conversion and Management, vol. 44, no. 9, pp. 1547-1561, 2003. [Online]. Available: https://doi.org/10.1016/S0196-8904(02)00166-8
[2] H. L. MacLean and L. B. Lave, "Evaluating automobile fuel/propulsion system technologies," Progress in Energy and Combustion Science, vol. 29, no. 1, pp. 1-69, 2003. [Online]. Available: https://doi.org/10.1016/S0360-1285(02)00032-1

[3] CEPAL, "Consideraciones ambientales en torno a los biocombustibles líquidos," in División de Desarrollo Sostenible y Asentamientos Humanos,, 2008.

[4] S. Fernández Henao, J. Mosquera A., and J. Mosquera M., "Análisis de emisiones de $\mathrm{CO}^{2}$ para diferentes combustibles en la población de taxis en Pereira y Dosquebradas," Scientia et Technica, vol. 2, no. 45, ago. 2010. [Online]. Available: https://doi.org/10.22517/23447214.385

[5] E. A. Melo Espinosa, Y. Sánchez Borroto, N. Ferrer Frontela, and N. Ferrer Frontela, "Evaluación de un motor de encendido por chispa trabajando con mezclas etanol-gasolina," Ingeniería Energética, vol. 33, pp. 94-102, 082012. [Online]. Available: https://bit.ly/38kOxFW

[6] J. Goñi Delión and M. Rojas-Delgado, "Combustibles alternativos en motores de combustión interna," Ingeniería Industrial, 02 2014. [Online]. Available: https://doi.org/10.26439/ing.ind2014. n032.122

[7] J. Gibbons, Interagency Assessment of Oxygenated Fuels. National Science and Technology Council, 1997. [Online]. Available: https://bit.ly/355W21N

[8] Apace Research Ltd, "Intensive field trial of ethanol/petrol blend in vehicles," ERDC Project No. 2511, Tech. Rep., 1998. [Online]. Available: https://bit.ly/32iZaFK

[9] R. Ragazzi and K. Nelson, "The impacts of a $10 \%$ ethanol blended fuel on the exhaust emissions of tier 0 andtier 1 light duty gasoline vehicles al 35 f," Colorado Department of Public Health and Environment, 1999.

[10] B.-Q. He, Jian-Xin Wang, J.-M. Hao, X.-G. Yan, and J.-H. Xiao, "A study on emission characteristics of an efi engine with ethanol blended gasoline fuels," Atmospheric Environment, vol. 37, no. 7, pp. 949-957, 2003. [Online]. Available: https://doi.org/10.1016/S1352-2310(02)00973-1

[11] T. W. Patzek, S.-M. Anti, R. Campos, K. W. ha, J. Lee, B. Li, J. Padnick, and S.-A. Yee, "Ethanol from corn: Clean renewable fuel for the future, or drain on our resources and pockets?" Environment, Development and Sustainability, vol. 7, no. 3, pp. 319-336, Sep. 2005. [Online]. Available: https://doi.org/10.1007/s10668-004-7317-4 
[12] C.-W. Wu, R.-H. Chen, J.-Y. Pu, and T.-H. Lin, "The influence of air-fuel ratio on engine performance and pollutant emission of an si engine using ethanol-gasoline-blended fuels," Atmospheric Environment, vol. 38, no. 40, pp. 7093-7100, 2004, 8th International Conference on Atmospheric Sciences and Applicat ions to Air Quality (ASAAQ). [Online]. Available: https://doi.org/10.1016/j.atmosenv.2004.01.058

[13] S. T. Coelho, J. Goldemberg, O. Lucon, and P. Guardabassi, "Brazilian sugarcane ethanol: lessons learned," Energy for Sustainable Development, vol. 10, no. 2, pp. 26-39, 2006. [Online]. Available: https://bit.ly/2JJIeBM

[14] R. K. Niven, "Ethanol in gasoline: environmental impacts and sustainability review article," Renewable and Sustainable Energy Reviews, vol. 9, no. 6, pp. 535-555, 2005. [Online]. Available: https://doi.org/10.1016/j.rser.2004.06.003

[15] American Coalition For Ethanol. (2005) Fuel economy study: comparing performance and costs of va-rious ethanol blends and standard unleaded gasoline. [Online]. Available: https://bit.ly/32mEmwP

[16] E. Behrentz, Beneficios ambientales asociados con el uso de combustibles alternativos. Centro de Investigaciones en Ingeniería Ambiental (CIIA) Universidad de los Andes, 2008. [Online]. Available: https://bit.ly/38k6cOj

[17] T. Durbin, J. W. Miller, T. Huai, D. R. Cocker III, and Y. Younglove, "Effects of ethanol and volatility parameters on exhaust emissions of light-duty vehicles." in UC Riverside: Center for Environmental Research and Technology. [Online]. Available: https://bit.ly/3oZFmkp

[18] E. Shapiro. (2006) Roundtable on ethanol fuel: automaker view.

[19] H. S. Yücesu, T. Topgül, C. Çinar, and M. Okur, "Effect of ethanol-gasoline blends on engine performance and exhaust emissions in different compression ratios," Applied Thermal Engineering, vol. 26, no. 17, 2006. [Online]. Available: https: //doi.org/10.1016/j.applthermaleng.2006.03.006
[20] H. R. Acevedo G. and J. M. Mantilla G., "Viabilidad ambiental del uso de biocombustibles para motores a gasolina y diésel en colombia," Boletin del Observatorio Colombiano de Energía, Bogota. D. C., pp. 3-14, 2008. [Online]. Available: https://bit.ly/38kSXg0

[21] U.S. Department of Energy, "Handbook for handling, storing, and dispensing e85 and other ethanol-gasoline blends," U.S. Department of Energy, Tech. Rep., 2013. [Online]. Available: https://bit.ly/2U1zVmF

[22] H. Gutiérrez Pulido and R. de la Vara Salazar, Análisis y diseño de experimentos. McGraw-Hill, 2003. [Online]. Available: https://bit.ly/36gB7rU

[23] E. A. García, "Modelización termodinámica de un motor turboalimentado y propulsado por bioetano," 2009. [Online]. Available: https://bit.ly/3k85tBO

[24] INEN, "NTE INEN 2478: Etanol anhidro. requisitos," Instituto Ecuatoriano de Normalización, Tech. Rep., 2009. [Online]. Available: https://bit.ly/354abwc

[25] — , "NTE INEN, Gasolina. Requisitos," Instituto Ecuatoriano de Normalización, Tech. Rep., 2012. [Online]. Available: https://bit.ly/2JLGsQD

[26] Hyundai. (2011) Manual del taller. [Online]. Available: https://bit.ly/356tPIc

[27] S. P. Verma and A. Quiroz-Ruiz, "Critical values for six Dixon tests for outliers in normal samples up to sizes 100, and applications in science and engineering," Revista Mexicana de Ciencias Geológicas, vol. 23, pp. 133-161, 01 2006. [Online]. Available: https://bit.ly/3eBoOKG

[28] W. Contreras, J. Ortega, and R. Japa, "Aplicación de una red neuronal feed-forward backpropagation para el diagnóstico de fallas mecánicas en motores de encendido provocado," INGENIUS, pp. 32-40, 2019. [Online]. Available: https://doi.org/10.17163/ings.n21.2019.03 


\title{
Algoritmos PARA EL RECONOCIMIENTo DE ESTRUCTURAS DE TABLAS Algorithms for Table Structure RECOGNITION
}

\author{
Yosveni Escalona Escalona ${ }^{1, *}$
}

Recibido: 03-08-2020, Revisado: 22-09-2020, Aprobado tras revisión: 09-10-2020

\section{Resumen}

Las tablas son una manera muy común de organizar y publicar datos. Por ejemplo, en el Internet se halla un enorme número de tablas publicadas en HTML integradas en documentos PDF, o que pueden ser simplemente descargadas de páginas web. Sin embargo, las tablas no siempre son fáciles de interpretar pues poseen una gran variedad de características y son organizadas en diferentes formatos. De hecho, se han desarrollado muchos métodos y herramientas para la interpretación de tablas. Este trabajo presenta la implementación de un algoritmo, basado en campos aleatorios condicionales (CRF, Conditional Random Fields), para clasificar las filas de una tabla como fila de encabezado, fila de datos y fila metadatos. La implementación se complementa con dos algoritmos para reconocer tablas en hojas de cálculo, específicamente, basados en reglas y detección de regiones. Finalmente, el trabajo describe los resultados y beneficios obtenidos por la aplicación del algoritmo para tablas HTML, obtenidas desde la web y las tablas en forma de hojas de cálculo, descargadas desde el sitio de la Agencia Nacional de Petróleo de Brasil.

Palabras clave: datos tabulados, tablas HTML, hoja de cálculo, campos aleatorios condicionales, aprendizaje automático

\section{Abstract}

Tables are widely adopted to organize and publish data. For example, the Web has an enormous number of tables, published in HTML, embedded in PDF documents, or that can be simply downloaded from Web pages. However, tables are not always easy to interpret due to the variety of features and formats used. Indeed, a large number of methods and tools have been developed to interpreted tables. This work presents the implementation of an algorithm, based on Conditional Random Fields (CRFs), to classify the rows of a table as header rows, data rows or metadata rows. The implementation is complemented by two algorithms for table recognition in a spreadsheet document, respectively based on rules and on region detection. Finally, the work describes the results and the benefits obtained by applying the implemented algorithm to HTML tables, obtained from the Web, and to spreadsheet tables, downloaded from the Brazilian National Petroleum Agency.

Keywords: Tabular Data, HTML Tables, Spreadsheets, Conditional Random Fields, Machine Learning, Algorithm.

\footnotetext{
$\overline{1, *}$ Research and Development Department, SOLINTEC, Araçatuba, Brazil

Autor para correspondencia yosveni.escalona@solinftec.com.br. (1) http://orcid.org/0000-0003-2992-0540
}

Forma sugerida de citación: Excalona Escalona, Y. (2021). «Algoritmos para el reconocimiento de estructuras de tablas». IngEniUs. N. ${ }^{\circ}$ 25, (enero-junio). pp. 50-61. DOI: https://doi.org/10.17163/ings.n25.2021.05. 


\section{Introducción}

El volumen de datos disponible en la Internet ha crecido de una manera vertiginosa, lo cual la ha convertido en un vasto repositorio de datos que describen nuestro ambiente y nuestras interacciones. La riqueza y fortaleza de estos datos permiten el desarrollo de la economía y la sociedad hoy en día.

Estos datos están relacionados con información de productos, artículos que imparten conocimiento enciclopédico, presentaciones de resultados científicos de avanzada o reportes sobre datos financieros actuales. Una gran parte de ellos pueden encontrarse en tablas, que requieren un análisis particular ya que pueden estar expresadas en HTML, integradas en documentos PDF o estar disponibles como hojas de cálculo descargables, entre otros formatos. Usualmente, las tablas se organizan de forma simple y compacta como filas y columnas, pero pueden ser más complejas con metadatos e información adicional.

Las tablas han demostrado ser fuentes valiosas, pero su uso puede estar muy diversificado, desde la búsqueda en la web hasta el descubrimiento de datos en hojas de cálculo y aumento de bases de conocimiento [1]. En la literatura se encuentran estudios sobre métodos y herramientas para la extracción de datos tabulares de hojas de cálculo, tablas HTML, tablas integradas en documentos PDF, etc. La gran mayoría de estos métodos y herramientas utilizan estrategias basadas en reglas heurísticas y algoritmos de aprendizaje de máquina. La estrategia para extraer datos tabulares y para clasificar filas de tablas también depende del formato del documento. Explorar un conjunto grande de tablas ha sido un reto porque, en general, la semántica de la tabla es desconocida. En [2], se presenta un corpus de más de cien millones de tablas, pero el significado de cada tabla raramente está explícito en la misma tabla. Otro reto es la estructura de la tabla. Por ejemplo, las tareas descritas en [3-6] se enfocan en recuperar la semántica de la tabla y en vincular sus datos con fuentes externas para tablas clasificadas como genuinas, con una pérdida considerable de datos. Estos trabajos no consideran aspectos fundamentales, tales como la orientación de la tabla y descartan aquellas tablas clasificadas como no genuinas.

Otro aspecto a considerar está basado en el tipo de documento, por ejemplo, Correa y Zander [7] analizaron un grupo de métodos y herramientas enfocados en extraer contenido tabular de archivos PDF basándose en dos características principales: facilidad de uso y resultados de salida y la categorización de las herramientas según propuestas teóricas, sin costo y comerciales. En [8] se desarrollaron varias heurísticas, que conjuntamente reconocen y descomponen tablas en archivos PDF y almacenan los datos extraídos en un formato estructurado de datos (XML) para facilitar su uso, estas heurísticas se dividen en dos grupos: reconocimiento y descomposición de tablas. Otras técnicas fueron presentadas en [9] para extraer data tabular de documentos PDF con el fin de identificar los límites de la tabla, donde los autores describen una metodología que aplica dos algoritmos de aprendizaje de máquina, CRF y máquinas de soporte vectorial (SVM, Support Vector Machines). Asimismo, se han revisado trabajos basados en el proceso de identificación de límites de tabla y diseñados para la correspondencia semántica y anotación de atributos numéricos y variantes en el tiempo en tablas web como las presentadas en [10-12] que anotan tablas web efectiva y eficientemente, e identifican los límites entre filas (o columnas) de nombres de atributos y sus correspondientes filas (o columnas) de valores en la tabla.

También se puede hacer mención especial de los trabajos relacionados con el reconocimiento de la estructura de una tabla HTML y la detección y clasificación del encabezado de una tabla descritas en $[13,14]$, sugiriendo algunas técnicas basadas en reglas heurísticas que utilizaron un algoritmo de aprendizaje de clasificación para delinear tipos de tablas existentes dentro de un documento y detectar los tipos de estructuras y encabezados.

Finalmente, se hace énfasis en el enfoque propuesto en [15] que estuvo basado en técnicas de aprendizaje de máquina que cubren dos tareas fundamentales del proceso de extracción de una tabla: su localización e identificación de las posiciones y tipos de filas. Este trabajo se enfoca en la implementación de dos algoritmos para el reconocimiento de tablas en hojas de cálculo, así como también otros algoritmos basados en campos aleatorios condicionales (CRF), para clasificar los tipos de filas dentro de las tablas. Los conjuntos de datos fueron creados con tablas HTML descargadas; las tablas de hojas de cálculo fueron obtenidas del sitio web de la Asociación Nacional de Petróleo (ANP) de Brasil.

\subsection{Contexto}

Las tablas se encuentran frecuentemente en documentos impresos, libros o periódicos, así como también en documentos digitales, páginas electrónicas o láminas de presentación. Sin embargo, dichas tablas también representan un concepto esencial en bases de datos relacionales y hojas de cálculo. Las tablas pueden distinguirse de acuerdo con su estructura y orientación. Una tabla relacional u horizontal [8], como la que se ilustra en la Tabla 1, tiene filas que proporcionan datos sobre objetos específicos llamados entidades y columnas, que representan atributos que describen las entidades. 
Tabla 1. Ejemplo de una tabla relacional

\begin{tabular}{ccccc}
\hline ID & Nombre & Edad & País & Ocupación \\
\hline 1 & Bob Smith & 35 & USA & Programador \\
2 & Jane Smith & 31 & USA & Profesora \\
3 & Robert White & 24 & UK & Ingeniero \\
\hline
\end{tabular}

Existen tablas más complejas, como aquellas donde los atributos que describen las entidades están colocados verticalmente y las entidades de manera horizontal, u otro tipo de estructuras como las que se muestran en la Tabla 2 y en la Tabla 3.

Tabla 2. Ejemplo de una tabla no relacional

\begin{tabular}{cccc}
\hline & Obj 1 & Obj 2 & Obj 3 \\
\hline Nombre & V1 & V1 & V1 \\
Edad & V2 & V2 & V2 \\
Altura & V3 & V3 & V3 \\
\hline
\end{tabular}

Tabla 3. Ejemplo de una tabla no relacional con información adicional

\begin{tabular}{lcc}
\hline \multicolumn{3}{c}{ Aplicaciones de patentes por residentes } \\
\hline \multicolumn{3}{c}{ Fuente de datos: worldbanc.org } \\
(se muestra país tope en cada continente)
\end{tabular}

De manera más precisa, una tabla se define como:

Definición 1. Una tabla es un par $\mathrm{T}=(\mathrm{H}, \mathrm{D})$ que consiste de un encabezado opcional $\mathrm{H}$ y datos $\mathrm{D}$, donde:

- El encabezado $H=\left\{h_{1}, h_{2}, \ldots, h_{n}\right\}$ es una n-tupla de elementos $h_{i}$ de encabezado; si el conjunto de elementos de encabezado existe, este podría ser representado como una fila o como una columna.

- Los nodos de datos están organizados como una matriz $(n, m)$ que consiste de $n$ filas y $m$ columnas:

$$
D=\left[\begin{array}{ccc}
C_{11} & \therefore \cdots & C_{1 m} \\
\vdots & \ddots & \vdots \\
C_{n 1} & \cdots & C_{n m}
\end{array}\right]
$$

El proceso de clasificación de las filas de una tabla consiste en identificar cada uno de los elementos de una tabla. La idea general está basada en localizar el encabezado y los datos en la tabla. También es relevante identificar los elementos de diseño y los metadatos. La Figura 1 muestra el proceso de clasificación de las filas de una tabla, denotando en diferentes colores algunos de los elementos presentes en la tabla: rojo indica los elementos que representan los títulos; amarillo, la fila de encabezado; azul, los datos de las filas; y verde, los metadatos adicionales.
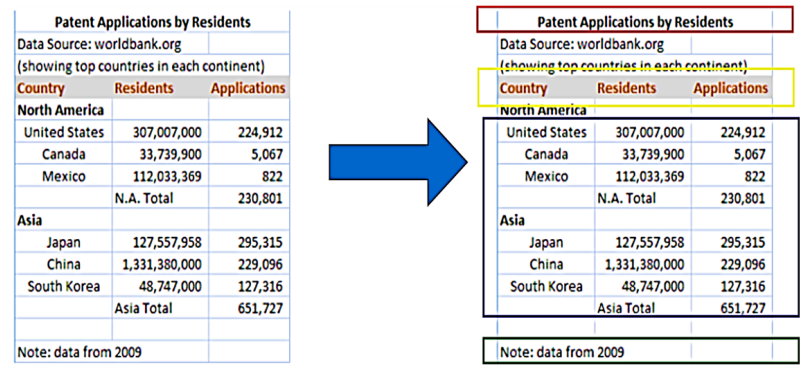

Figura 1. Proceso de extracción tabular

El resto del artículo está organizado como sigue. La sección 2 cubre detalles de las implementaciones de algoritmos para el reconocimiento de tablas y para la clasificación de filas de tablas. Finalmente, la sección 3 describe experimentos y resultados.

\section{Materiales y métodos}

\subsection{Algoritmos de reconocimiento de tablas y de clasificación de filas de tablas}

Esta sección describe las implementaciones de un algoritmo para reconocer tablas en hojas de cálculo y un algoritmo, basado en campos aleatorios condicionales, para clasificar filas de tablas.

\subsubsection{Un algoritmo basado en reglas para detectar tablas en hojas de cálculo}

Varios algoritmos basados en reglas detectan tablas en hojas de cálculo utilizando atributos de celdas, tales como bordes, formato y tipo de dato. El atributo de cada celda en la hoja de cálculo tiene un valor específico asociado con esa celda. A su vez, el borde de la celda tiene los atributos de dirección, estilo y color. El borde puede rodear la celda en 4 direcciones diferentes: arriba, abajo, izquierda y derecha.

Un formato de celda es el formateo visual aplicado al dato de la celda, tal como, formato de número, nombre de estilo de letra, nombre de letra, tamaño de letra, letra negrita, letra cursiva y color de letra. La detección de múltiples tablas en la misma hoja de cálculo se realiza encontrando un separador entre dos tablas (usualmente un conjunto de filas vacías), como se explica a continuación [16].

Dada una tabla $\mathrm{T}$, con $r n$ filas y $c n$ columnas, se calculan las siguientes características de diseño: 
- Número promedio de columnas, calculado como el número promedio de celdas por fila.

$$
c=\frac{1}{r n} \sum_{i=1}^{r n} c_{i}
$$

donde $c_{i}$ es el número de celdas en la fila $i$, $i=1, \ldots r n$.

- Número promedio de filas, calculado como el número promedio de celdas por columna.

$$
r=\frac{1}{c n} \sum_{i=1}^{c n} r_{i}
$$

donde $r_{i}$ es el número de celdas en la columna $i$, $i=1, \ldots, c n$.

La Figura 2 muestra el algoritmo que identifica el número de tablas dentro de un documento y captura el rango de filas que representa cada tabla.

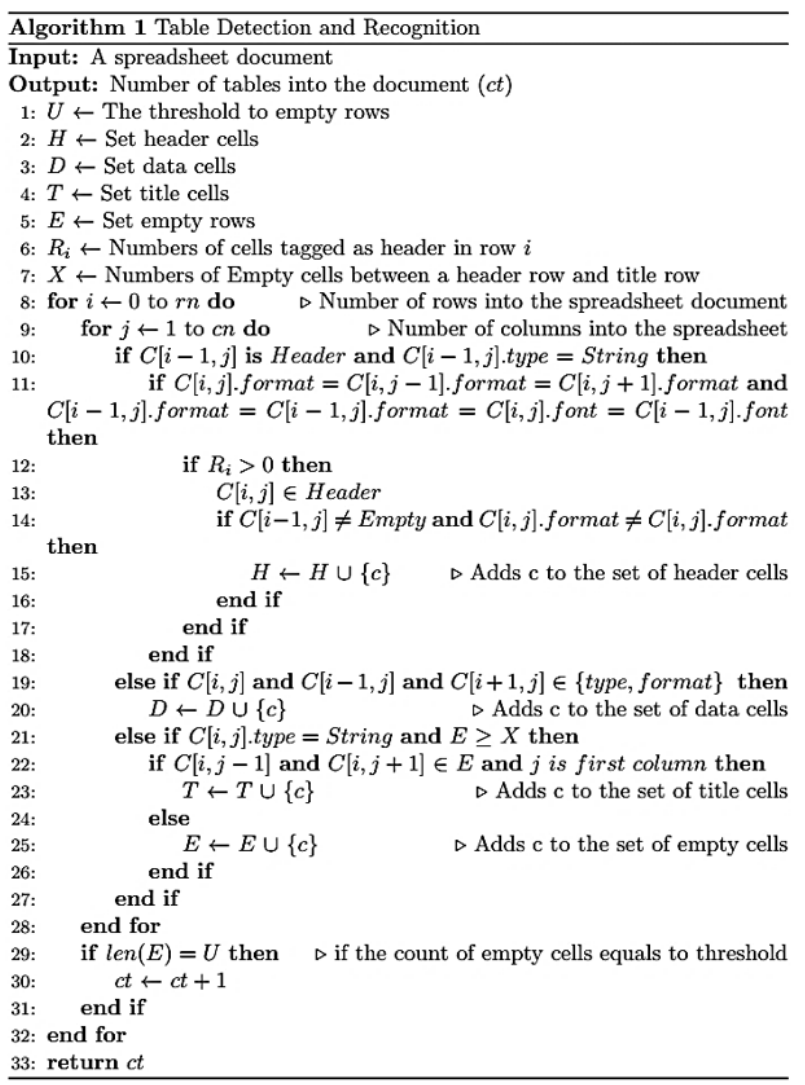

Figura 2. Algoritmo de detección y reconocimiento de tabla

\section{Detección de regiones}

La detección de regiones se calcula a través de un algoritmo basado en grafos denominado Remover y Conquistar [17], que detecta tablas en hojas de cálculo.
Este algoritmo utiliza un conjunto completo de reglas y heurísticas de acuerdo con una representación de una hoja de cálculo como un grafo. Los archivos de hojas de cálculo contienen una o más hojas, cada hoja consta de una colección de celdas organizadas en filas y columnas, donde se definen ciertos términos útiles para el proceso de detección de regiones.

Definición 2. Sea $W$ el conjunto que contiene todas las celdas de una hoja.

La detección de una región consiste en escanear la hoja de cálculo desde la primera celda en la esquina superior izquierda hasta la última celda no vacía en la esquina inferior derecha, para chequear celdas con formato similar y detectar separadores, tales como filas vacías, diferentes formatos de celdas o diferentes tipos de bordes, tales como diferentes tipos de valores de celdas. De manera más precisa, una región se define como:

Definición 3. Una región es una colección máxima $R \subseteq W$ de celdas de un área rectangular de la hoja.

También se infiere el rol de diseño de las celdas no vacías en la hoja, donde a cada celda no vacía se le asignan los siguientes roles: Encabezado (H, Header), Datos (D), Título (T), Metadato o no relacional (N). Este rol de celda se define como sigue.

Definición 4. Sea la label: $W \rightarrow$ Etiquetas, donde Etiquetas $=\{$ Encabezado, Datos, Título, Metadatos $\}$, una función que relaciona a las celdas su rol de diseño asignado. Para celdas vacías, la etiqueta no está definida; estas celdas se identifican utilizando empty: $W \rightarrow\{0,1\}$, que retorna 1 para celdas vacías y 0 en otro caso.

Las celdas de una hoja de cálculo se agrupan conjuntamente, de manera que celdas adyacentes tengan el mismo rol de diseño (etiqueta) o formen estructuras más grandes. Estos grupos se denominan regiones etiqueta, como se muestra en [17] y en la Figura 3.
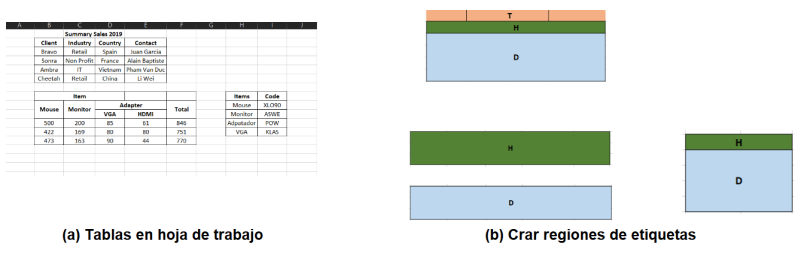

Figura 3. Proceso de creación de regiones etiquetas

Formalmente, una región etiqueta se define de la siguiente manera:

Definición 5. Una región etiqueta es una región $L R$ de una hoja de cálculo tal que, para cualesquiera dos celdas $c$ y $c^{\prime}$ en $L R$, label $(c)=\operatorname{label}\left(c^{\prime}\right)$ y empty $(c) \neq 1$ $\mathrm{y} \operatorname{empty}\left(c^{\prime}\right) \neq 1$.

La Figura 4(a) muestra tablas en una hoja de cálculo y la Figura 4(b) indica las regiones correspondientes a las estructuras de la tabla. El proceso de detección de regiones etiqueta agrupa celdas según su 
etiqueta. Se itera a lo largo de cada fila para crear secuencias de celdas que tienen la misma etiqueta. Estas forman la base de LR. Posteriormente, se unen las $L R$ de filas consecutivas, si sus etiquetas, columna mínima y columna máxima coinciden.

\section{Representación de tablas mediante grafos}

Las regiones permiten construir grafos que capturan las interrelaciones de regiones etiqueta. La Figura 4 muestra la representación de tablas como un grafo.
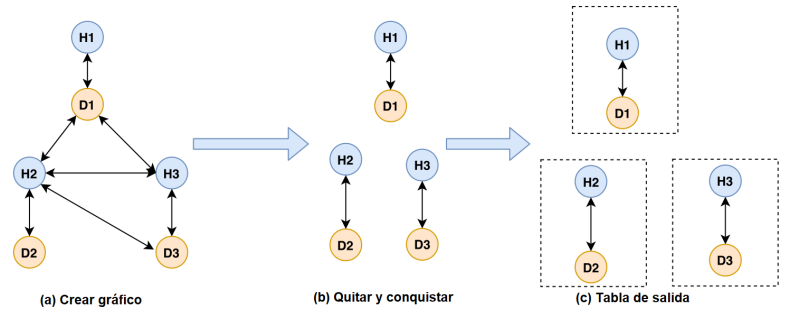

Figura 4. Representación de tablas mediante grafos

El proceso de construcción de grafos consiste en identificar relaciones espaciales: superior, inferior, izquierda y derecha, localizando las regiones vecinas más cercanas para cada dirección, e identificar todos los vértices cuya máxima fila es menor que la mínima fila de otro vértice. Para cada dirección, se define una función distancia donde se identifican todos los vértices más cercanos:

$$
N D_{v}=\left\{n \in D_{v} \vee \operatorname{ddist}(v, n)=\operatorname{mind}_{u \in D V} \operatorname{dist}(u, v)\right\}
$$

donde $D_{v}$ es la dirección para el vértice $v$; bordes dirigidos $(v, n)$ se crean para cada $n \in N D_{v}$.

\subsubsection{Algoritmo Remover y Conquistar}

Remover y Conquistar (RAC, Remove and Conquer) es un algoritmo basado en reglas cuyo objetivo es separar los bordes que están más lejos hacia las direcciones izquierda y derecha del grafo que fue creado a partir de cada hoja en una hoja de cálculo, como se muestra en la Figura 5. El algoritmo procesa los componentes fuertemente conectados del grafo, para aparear todos los grupos formados y detectar tablas válidas.

Los vértices se ordenan en orden descendente de su máxima fila, seguido por orden ascendente de su mínima fila, por lo que las tablas se buscan en orden inverso, desde abajo hacia arriba. Cada encabezado $\mathrm{h}$ se procesa individualmente para identificar vértices con fila mínima mayor o igual que $h$.

El algoritmo que verifica el encabezado válido se muestra en la Figura 6 . Todos los encabezados válidos son almacenados en $Q$ que representa el conjunto de vértices, incluyendo $h$; este conjunto de vértices se denomina tablas potenciales. El algoritmo asegura que otros vértices conectados a h no se dejen aislados.

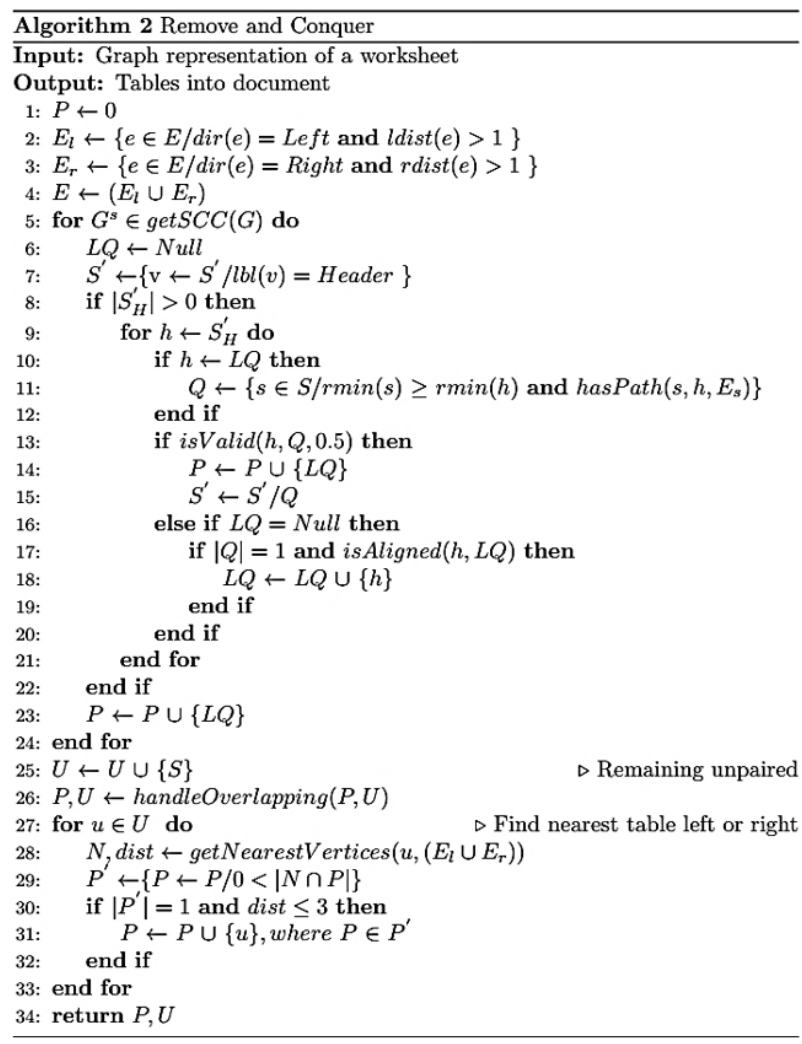

Figura 5. Algoritmo Remover y Conquistar

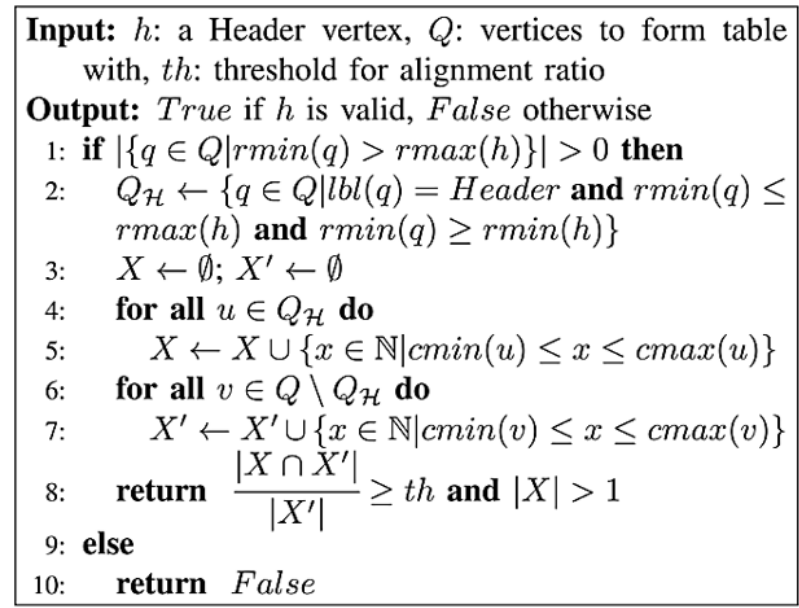

Figura 6. Chequeo de validez del encabezado

Esos vértices apareados con un encabezado válido son sustraídos del conjunto de vértices y luego ordenados para crear el conjunto S'. Los encabezados válidos son agregados al conjunto de encabezados válidos, llamado $L Q$. Los vértices que representan tablas potenciales, llamados $Q$, no son directamente agregados al conjunto de tablas $P$ porque el algoritmo necesita 
chequear que h no está conectado a otros vértices. Las tablas que no pueden formarse se almacenan en $U$. Entonces, en el último paso del algoritmo, este intenta aparear las tablas en $U$ con la tabla más cercana a su izquierda o derecha.

\subsubsection{Un algoritmo de aprendizaje de máquina para clasificar filas de tabla}

Una contribución importante de este trabajo es la identificación y clasificación de los tipos de filas que componen una tabla, a través de la implementación de un algoritmo de aprendizaje de máquina, en este caso, campos aleatorios condicionales (CRF), el cual está basado en las características, valores de las celdas, así como también las clases que representan la estructura de la tabla.

Los CRF son modelos de grafos que no tienen dirección, introducidos por Lafferty et al. [18], pueden actuar como clasificadores en tareas de etiquetado de secuencias. Estos son utilizados frecuentemente para procesamiento de lenguaje natural, tal como etiquetado de partes de discursos. El algoritmo CRF define $\mathrm{X}$ como una variable aleatoria sobre las secuencias de datos a ser etiquetadas y $\mathrm{Y}$ como una variable sobre las secuencias de etiquetas correspondientes. La Figura 7 muestra una estructura de un campo aleatorio condicional lineal.

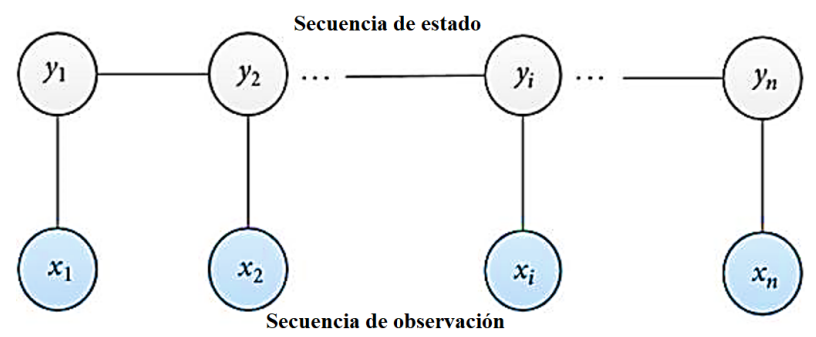

Figura 7. Estructura de un campo aleatorio condicional de cadena lineal

En nuestro problema de clasificar filas de tablas, la secuencia de entrada $x$ corresponde a una serie de filas de una tabla dada, mientras que la secuencia de etiquetas y es la serie de etiquetas asignadas a las filas observadas. A cada fila en $x$ se le asigna exactamente una etiqueta en $y$.

Formalmente, los campos condicionales aleatorios se definen de la siguiente manera:

Definición 6. Sea $G=(V, E)$ un grafo y $Y=\left(Y_{v}\right)_{v \in V}$ una secuencia de variables aleatorias indexadas por los vértices de $G$. Un campo aleatorio condicional es un par $(X, Y)$ tal que, cuando se condiciona en $X$, las variables aleatorias $Y_{v}$ obedecen la propiedad de Markov con respecto al grafo.

$$
P\left(Y_{v} \mid X, Y_{w}, w \neq v=P\left(Y_{v} \vee X, Y_{w} \approx v\right)\right.
$$

$$
\begin{array}{r}
P(X \vee Y)=\frac{1}{Z(x)} \exp \left(\sum_{j} \lambda_{j} f_{j}\left(Y_{i-1}, Y_{i}, X, i\right)+\right. \\
\left.+\sum_{k} \mu_{k} g_{k}\left(Y_{i}, X, i\right)\right)
\end{array}
$$

donde $f_{j}\left(Y_{i-1}, Y_{i}, X, i\right)$ es una función de características de transición de la secuencia de observaciones y de las etiquetas en las posiciones $i$ e $i-1$ en la secuencia de etiquetas; $g_{k}\left(Y_{i}, X, i\right)$ es una función de características de estado de la etiqueta en la posición $i$ y la secuencia de observaciones; y $\lambda_{j}$ y $\mu_{k}$ son parámetros a ser estimados a partir de datos de entrenamiento.

En el escenario de la tabla de datos, $X$ representa la lista de filas en la tabla y $Y$ representa las clases de fila correspondientes. Cada tabla de datos relacional tiene un esquema, el cual, en el contexto de las tablas de datos, consiste de nombres de atributos, valores y tipos, donde los nombres de atributos son títulos de columnas, los tipos de atributos son los tipos de valores en la columna y los atributos de valor corresponden a valores de datos en las celdas de las columnas. Los nombres de las columnas son almacenados en una fila o en filas especiales, usualmente cerca del encabezado de la tabla, llamadas filas de encabezado, mientras que los datos son almacenados en filas referidas como filas de datos.

La tabla de datos también puede contener descripciones de datos referidas a los metadatos. En correspondencia con los criterios tratados arriba, se identifica cada tipo de fila de acuerdo con las propiedades de cada celda en la tabla de datos. Entonces, el problema se enfoca en asignar una etiqueta a cada fila, donde cada fila está constituida por celdas que pueden exhibir diferentes conjuntos de atributos. El proceso de selección de características involucra la extracción de una colección de atributos para celdas individuales y combinar los atributos de todas las celdas en la fila, con el fin de construir un conjunto de características de filas. Considere las ideas abordadas arriba y un ejemplo de una tabla simple con encabezado y datos, tal como se muestra en la Figura 8.

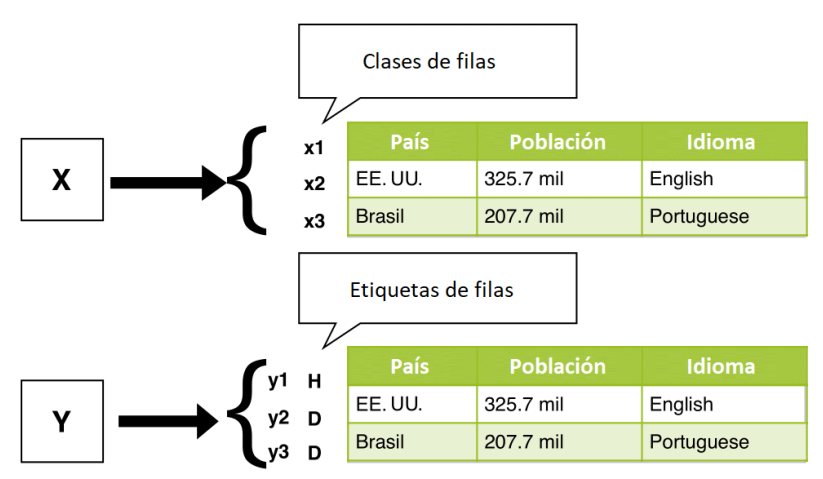

Figura 8. Ejemplo de una tabla etiquetada 
$X$ representa un vector con las filas de la tabla y $Y$ representa otro vector con las etiquetas de cada fila $x$ de la tabla.

\subsubsection{Clases de filas}

De acuerdo con la estructura de tablas y la Definición 6 , los tipos de clases de filas que se muestran en la Tabla 4.

Tabla 4. Clases de filas

\begin{tabular}{cc}
\hline Etiqueta & Descripción \\
\hline H & Representa la fila de encabezado en la tabla \\
D & Filas de datos que contienen registros de datos \\
N & Metadatos no relacionales \\
\hline
\end{tabular}

\subsubsection{Conjunto de características}

En cualquier algoritmo de aprendizaje de máquina, una característica es una propiedad individual medible o una característica de un fenómeno que está siendo observado [19]. Por lo tanto, cada característica se particiona en tres categorías considerando aspectos relacionados al diseño, estilos y valores que pueden llamarse atributos de diseño.

Atributos de diseño son las celdas que se encuentran comúnmente en filas de encabezado, que usualmente contienen celdas de tablas combinadas con texto centrado.

Atributos de estilo son varias propiedades derivadas de hojas de estilo, tales como tipo de letra, color de letra, peso de letra o texto subrayado.

Atributos de valor son aquellos que representan celdas donde la información almacenada está vinculada exclusivamente con las filas de datos. Frecuentemente, las filas de encabezado contienen valores textuales relativamente cortos, en lugar de números o fechas.

Observe con un ejemplo como trabaja el algoritmo CRF en nuestro problema de clasificación de tabla, dada la característica de transición $f_{j}\left(Y_{i-1}, Y_{i}, X, i\right)$ y la función de características $g_{k}\left(Y_{i}, X, i\right)$ :

$x$ es una fila dentro de la tabla de datos.

$j$ is la posición de una fila en la tabla (cada característica está asociada con una posición); más de una característica asociada con la misma posición.

$y_{j-1}, y_{j}$ son las etiquetas (clases) asignadas a las filas $j$ y $j-1$ de $x$

Entonces, la función de características y la función de estados son las siguientes:

$$
f_{1}\left(Y_{i-1}, Y_{i}, X, i\right)=\left\{\begin{array}{c}
1 i f x_{j} \in \text { headery } \\
0, \text { en otro caso }
\end{array}\right.
$$

$$
f_{2}\left(Y_{i-1}, Y_{i}, X, i\right)=\left\{\begin{array}{c}
1 \text { if }_{j} \in \text { datay }_{j}=D \\
0, \text { en otro caso }
\end{array}\right.
$$

$$
\begin{array}{r}
g_{1}\left(Y_{i}, X, i\right)= \\
\left\{\begin{array}{c}
1 i f\left(x_{j} \text { isacell } \in x\right) \wedge\left(x_{j} \in \text { rowfeatures }\right) \wedge y_{j} \\
0, \text { en otro caso }
\end{array}\right.
\end{array}
$$

$$
\begin{array}{r}
g_{2}\left(Y_{i}, X, i\right)= \\
\left\{\begin{array}{r}
1 i f\left(x_{j} \text { isacell } \in x\right) \wedge\left(x_{j} \in \text { rowfeatures }\right) \wedge y_{j}=D \\
0, \text { en otro caso }
\end{array}\right.
\end{array}
$$

La lista completa de atributos de celdas individuales está dada en la Tabla 5. Las características se dividen de según el tipo de atributos que ellas representan.

Tabla 5. Atributos de celdas

\begin{tabular}{cccc}
\hline Diseño & Estilo & Valor & Espacial \\
\hline IsMerged & IsBold & IsEmpty & RowNumber \\
Aligment & IsItalic & IsText & ColNumber \\
& IsUnderlined & IsNumber & NumNeighbor \\
& IsColored & IsDate & MatchStyle \\
& Font & IsAlpNum & MatchType \\
& Format & IsCapital & \\
& Border & TotalWord & \\
\hline
\end{tabular}

\subsubsection{Similitud entre filas}

Otra característica que fue tomada en cuenta para generalizar los datos de entrenamiento fue la similitud entre filas [20], donde se asigna una característica única a cada combinación única $(c, r)$ donde $c$ es el número de celdas que exhiben un atributo y $r$ es el número de celdas en la fila. Entonces, dos filas $R_{x}$ y $R_{y}$ se consideran similares con respecto a cierto atributo de celda $\alpha$ si el logaritmo de sus anchuras son iguales y el logaritmo del número de celdas que exhiben o carecen del atributo $\alpha$. Este enfoque se conoce como agrupación de características y puede definirse como sigue.

Formalmente, para una fila $R_{i}$ de longitud $r$ en la cual $c$ celdas exhiben un atributo específico de celda $\alpha$, se la asigna la característica " $R_{\alpha}=(a, b)$ " a $R_{j}(a, b)$, donde $a$ y $b$ son los grupos que se calculan como sigue.

$$
\begin{gathered}
a=\left\{\begin{array}{cc}
0, & \text { if } c=0 \\
\left\lfloor\log _{2}(c)+1\right\rfloor, & \text { if } 0<\leq r / 2 \\
\left\lfloor\log _{2}(r-c)+1\right\rfloor, & \text { if } r / 2<c<r \\
0^{-} & \text {if } c=r
\end{array}\right. \\
b=\left\lfloor\log _{2}(r)\right\rfloor
\end{gathered}
$$


Los objetivos de los grupos son:

1. Diferenciar entre anchos de tablas

2. Combinar tablas anchas

3. Resaltar filas uniformes

\section{Resultados y discusión}

Esta sección presenta los experimentos realizados para probar la exactitud de la implementación del clasificador de filas de tablas, así como también los experimentos con reconocimientos de tablas en documentos con hojas de cálculo.

\subsection{Preprocesamiento}

La tarea de preprocesamiento se enfoca en dos escenarios de tablas: tablas HTML y tablas de hojas de cálculo, con el fin de remover contenido irrelevante o contenido que no proporcionará información para el clasificador de filas de tablas. Otros aspectos que fueron considerados fueron las estructuras de las tablas y la información presente en ambos tipos de tablas. Este trabajo no cubre en completo detalle el preprocesamiento de las tablas, por lo que solo se hace énfasis en aquellos elementos que se consideran más importantes. En el caso de tablas de hojas de cálculo, se resalta que el conjunto de datos tenía una anotación predefinida, pero con muchos errores relacionados con la identificación de los rangos de filas de datos y filas de encabezado.

\subsection{Características principales de los conjuntos de datos utilizados para prueba}

La Tabla 6 muestra las estadísticas del proceso de anotación en ambos conjuntos de datos. Cada fila de cada tabla fue anotada con la etiqueta correspondiente a su clase: «H» para encabezamiento (Header), «D» para datos, etc.

Tabla 6. Tablas anotadas

\begin{tabular}{ccc}
\hline & HTML & Hoja de cálculo \\
\hline Tablas anotadas & 105 & 252 \\
Filas anotadas & 13,025 & 227,638 \\
Filas de encabezado & $105(<1 \%)$ & $252(<1 \%)$ \\
Filas de datos & $12,920(99 \%)$ & $227,254(98 \%)$ \\
Otras clases de filas & $0(0 \%)$ & $132(<1 \%)$ \\
\hline
\end{tabular}

La tabla indica que un aspecto crítico de tablas tanto HTML como hojas de cálculo es que el porcentaje de filas de encabezado es muy bajo, debido al hecho de que las tablas obtenidas fueron tablas simples con esquemas simples (tablas con una sola fila de encabezado seguida por una o más filas de datos).

\subsection{Experimentos de clasificación de tablas}

Esta sección presenta los experimentos para evaluar la solución propuesta de clasificación de tablas. En una primera etapa, se entrenó el algoritmo con $80 \%$ de los datos y se probó con $20 \%$ de los datos, seleccionados aleatoriamente. Se utilizó el algoritmo L-BFGS como método de optimización y parámetros de regularización L1 y L2 ajustados a 0.1 y 0.01 . Los experimentos con tablas HTML y hojas de cálculo fueron realizados de manera separada, para exponer las diferencias entre los dos formatos de tabla. Las métricas de desempeño adoptadas fueron precisión, memoria, f1-score, soporte.

\subsubsection{Resultados}

Esta sección muestra los resultados obtenidos. Se observa que el valor de precisión para tablas de hojas de cálculo fue mayor que para tablas HTML, debido a dos factores principales: (1) las características de las tablas de hojas de cálculo tienen una mejor definición; (2) se garantiza una correcta definición para las filas de datos. La memoria fue similar para ambos tipos de tablas, así como también el f1-score. Un punto importante en este análisis está relacionado con el número de filas clasificadas como no relacionales en el conjunto de datos de hojas de cálculo, debido al hecho de que se anotaron manualmente las tablas de hojas de cálculo, a diferencia de las HTML, donde algunas filas podrían haber sido identificadas como filas de datos o filas de encabezado, siendo de hecho filas no relacionales (Tabla 7).

Tabla 7. Resultados para tablas HTML y hojas de cálculo

\begin{tabular}{ccccc}
\hline Clase de fila & Precisión & Memoria & F1-Score & Soporte \\
\hline \multicolumn{5}{c}{ HTML } \\
\hline D & 0.966 & 0.982 & 0.970 & 2,496 \\
H & 0.955 & 0.992 & 0.970 & 17 \\
N & 0.980 & 0.980 & 0.970 & 92 \\
\hline \multicolumn{5}{c}{ Hojas de cálculo } \\
D & 0.997 & 0.985 & 0.994 & 39,08 \\
H & 0.969 & 0.993 & 0.983 & 49 \\
\hline
\end{tabular}

Nota: las etiquetas de fila son como en la Tabla 4:

D: Filas de datos

$\mathrm{H}$ : Filas de encabezado

$\mathrm{N}$ : Metadatos no relacionales (una nota, clarificación, etc.) 


\subsubsection{Validación cruzada}

Validación es el proceso de decidir si los resultados numéricos que cuantifican las hipótesis entre variables son aceptables como descripciones de los datos; este es un proceso útil cuando no existen datos suficientes para entrenar el modelo y existe una gran desequilibrio en el número de objetos en cada clase. Entonces, se aplicó una estrategia $k$-fold conocida como $k$-fold estratificado, que es una ligera variación de la estrategia $k$-fold de validación cruzada, tal que el fold contiene aproximadamente el mismo porcentaje de muestras de cada clase objetivo que el conjunto completo.

La Tabla 8 muestra los resultados obtenidos para ambos conjuntos de datos. Se observa que para el caso de tablas HTML, los mejores resultados se obtuvieron para $\mathrm{k}=2 \mathrm{y} \mathrm{k}=3$ y que la precisión promedio fue 0,958 y que para las tablas de hojas de cálculo cada $\mathrm{k}=1 \ldots, 5$ es similar y la precisión promedio fue 0,997 .

Tabla 8. Precisión del método de validación cruzada para tablas HTML y hojas de cálculo

\begin{tabular}{cccccc}
\hline \multicolumn{7}{c}{ HTML } \\
\hline Etapa & $\mathbf{K}=\mathbf{1}$ & $\mathbf{K}=\mathbf{2}$ & $\mathbf{K}=\mathbf{3}$ & $\mathbf{K}=\mathbf{4}$ & $\mathbf{K}=\mathbf{5}$ \\
Prueba & 0,92 & 0,98 & 0,98 & 0,94 & 0,97 \\
\hline \multicolumn{7}{c}{ Hojas de cálculo } \\
\hline Etapa & $\mathbf{K}=\mathbf{1}$ & $\mathbf{K}=\mathbf{2}$ & $\mathbf{K}=\mathbf{3}$ & $\mathrm{K}=\mathbf{4}$ & $\mathbf{K}=\mathbf{5}$ \\
Prueba & 0,997 & 0,998 & 0,996 & 0,998 & 0,996 \\
\hline
\end{tabular}

\subsubsection{Matriz de confusión}

Como en cualquier problema de clasificación, existen aspectos que pueden ser mejorados. En nuestros experimentos, se tienen que examinar las filas en cada clase que fueron confundidas con filas en otra clase. Entonces, se utilizó una matriz de confusión, como se muestran en la Figura 9 y en la Figura 10. Cada celda de la matriz muestra el porcentaje de todas las filas clasificadas que fueron realmente de la clase con la etiqueta mostrada en la primera columna, pero que el clasificador le asignó la etiqueta de fila mostrada en la primera fila. Las celdas sombreadas con color azul más oscuro en la diagonal muestran clasificaciones correctas de filas, mientras que las remanentes muestran clasificaciones incorrectas.

Idealmente, nuestro clasificador resultaría en ceros para los valores fuera de la diagonal. Sin embargo, de hecho, el modelo clasificó filas incorrectamente. En el caso de tablas de hojas de cálculo, se observó que, tanto para filas de datos como para filas de encabezado, se obtuvieron resultados erróneos con respecto a las filas no relacionales, esto es, un número considerable de filas de datos y filas de encabezado fueron identificadas como filas no relacionales. En las tablas HTML, los resultados erróneos para filas no relacionales fueron mayores que para las tablas de hojas de cálculo, siendo
$7,9 \%$ y $6,6 \%$ para filas de datos y filas de encabezado, respectivamente.

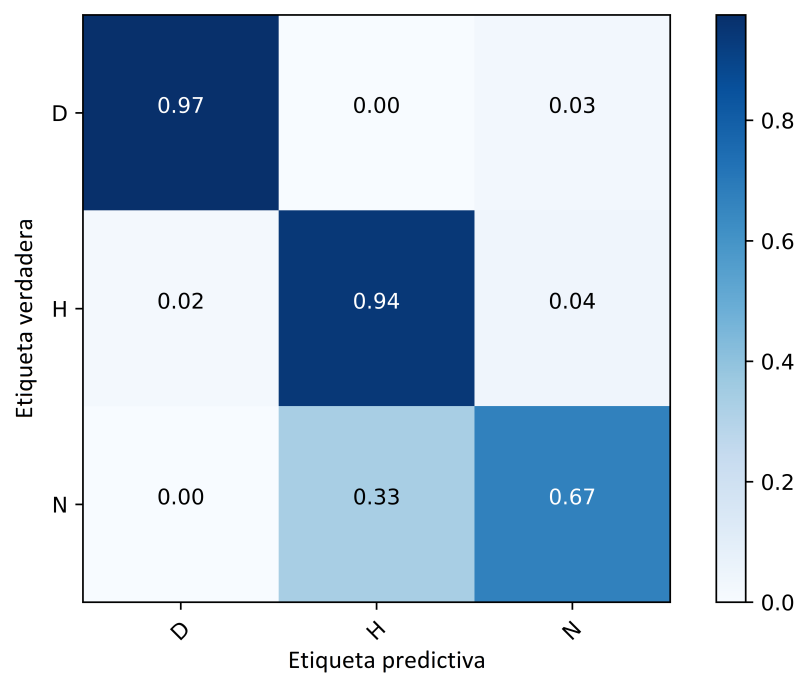

Figura 9. Matriz de confusión para tablas de hojas de cálculo

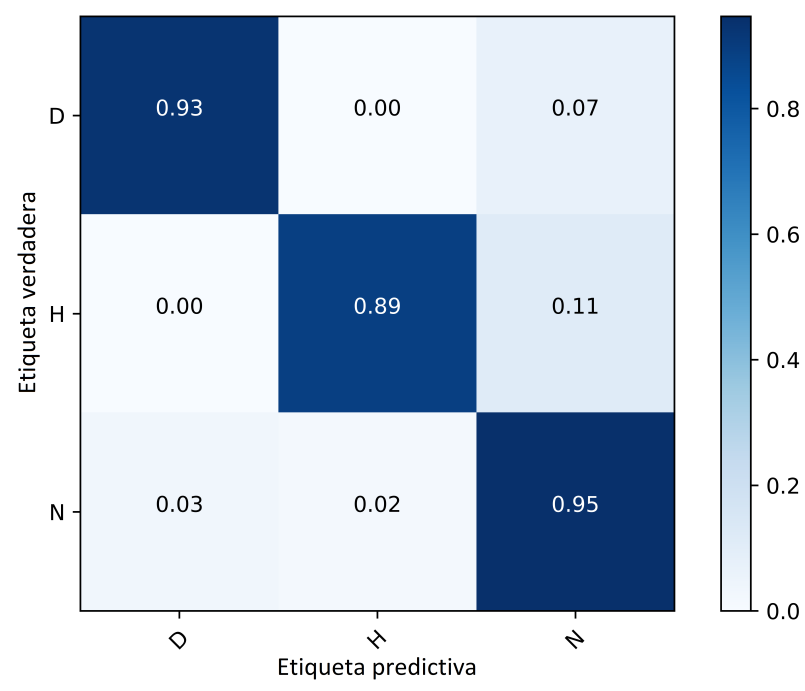

Figura 10. Matriz de confusión para tablas HTML

Esto merece alguna explicación: (1) la diferencia entre el número promedio de filas de las tablas HTML y el número promedio de filas de tablas de hojas de cálculo; (2) en nuestro proceso de clasificación, una fila dada se clasifica como «metadatos no relacionales» cuando la fila no puede ser identificada como de datos o de encabezado; (3) las tablas de hojas de cálculo tienen una mejor definición en términos de características, por ejemplo, las tablas dependen de propiedades encapsuladas dentro de archivos CSS. 


\subsection{Algoritmo basado en reglas para detección de tablas}

$\mathrm{El}$ algoritmo basado en reglas fue aplicado a hojas en un conjunto muestra que contenía tablas con diferentes diseños y gráficos integrados. La Tabla 9 resume los resultados obtenidos, que analizaron un total de 1000 documentos de hoja de cálculo, detectaron 1481 tablas y clasificaron incorrectamente 141.

Tabla 9. Resultados para el algoritmo de detección basado en reglas

\begin{tabular}{cc}
\hline Documentos de hojas de cálculo & $\mathbf{1 0 0 0}$ \\
\hline Tablas & 1481 \\
Tablas clasificadas incorrectamente & 141 \\
Tablas simples & 700 \\
Tablas múltiples & 158 \\
\hline
\end{tabular}

El algoritmo falló para tablas múltiples con separadores internos que son menores que el umbral definido. En ese caso, el algoritmo consideraría las dos tablas como una tabla simple. Asimismo, no reconocería correctamente tablas cuando las celdas no tengan atributos o separadores (por ejemplo, una tabla sin bordes, sin formato de letra, sin colores de fondo y sin filas vacías que separen encabezados y el título de la tabla) y no descubrió tablas donde el número de celdas vacías a la derecha e izquierda es extremadamente grande.

\subsubsection{Experimentos con el algoritmo de detección de tabla Remover y Conquis- tar}

El algoritmo Remover y Conquistar (RAC) fue aplicado al mismo conjunto de datos. Este algoritmo detectó tablas que no pudieron ser reconocidas por el algoritmo basado en reglas y maximizó la coincidencia entre la tabla propuesta $\mathrm{P}$ y la tabla verdadera $\mathrm{T}$, lo cual es equivalente a maximizar el número de celdas que ellas tienen en común y minimizar el número de celdas por las cuales difieren. La Tabla 10 muestra los resultados al comparar con el algoritmo 1, donde se observa que el número de tablas clasificadas incorrectamente se redujo y el número de tablas múltiples detectadas aumentó.

Tabla 10. Tablas reconocidas mediante RAC

\begin{tabular}{cc}
\hline Documentos de hojas de cálculo & 1000 \\
Tablas & 1481 \\
Tablas clasificadas incorrectamente & 141 \\
Tablas simples & 650 \\
Tablas múltiples & 230 \\
\hline
\end{tabular}

\subsection{Resultados de los algoritmos y descripción del ambiente}

Antes de entrar en detalle acerca de los tiempos de ejecución de los algoritmos, se explicarán las características principales del ambiente: computadora portátil (PC) modelo Lenovo $80 \mathrm{YH}$ con 8 GB de memoria RAM, procesador Intel(R) Core i7-7500 con $2.70 \mathrm{GHz}$, tarjeta gráfica Intel(R) 620 con $128 \mathrm{MB}$ de memoria, sistema operativo Windows 10 Home de 64 bits, la Tabla 8 muestra los tiempos de ejecución de cada uno de los algoritmos.

Tabla 11. Tiempo de ejecución de los algoritmos de reconocimiento de tablas

\begin{tabular}{|c|c|c|c|}
\hline Algoritmo & $\begin{array}{c}\text { Tiempo de } \\
\text { ejecución (s) }\end{array}$ & CPU (\%) & Memoria(\%) \\
\hline $\begin{array}{l}\text { Remover y } \\
\text { Conquistar }\end{array}$ & 114,28 & 4,3 & 1,3 \\
\hline Basado en reglas & 69,19 & 3,7 & 1 \\
\hline $\begin{array}{l}\text { Campo aleatorio } \\
\text { condicional }\end{array}$ & 376,57 & 11,5 & 2,5 \\
\hline
\end{tabular}

\section{Conclusiones}

En este trabajo se ha descrito la implementación de tres algoritmos para clasificar filas de una tabla y reconocer tablas en documentos de hojas de cálculo, respectivamente. Se realizaron experimentos para probar el desempeño del clasificador de filas de tablas utilizando tablas HTML y de hojas de cálculo. Los experimentos muestran que el clasificador obtuvo excelentes resultados para ambos tipos de tablas. Asimismo, se aplicó una validación cruzada $k$-fold donde se obtuvieron resultados similares a los otros experimentos reportados en [20].

En resumen, las contribuciones de este trabajo fueron:

- Un clasificador de filas de tabla, aplicable tanto a tablas HTML como a tablas de hojas de cálculo.

- Experimentos para validar el clasificador.

- Dos conjuntos de datos que contienen tablas HTML y de hojas de cálculo anotadas, para entrenar y validar clasificadores de filas de tablas.

- La implementación de dos algoritmos para el reconocimiento de tablas en documentos de hojas de cálculo.

Como trabajo futuro, se propone incrementar el número de instancias y clases en nuestros conjuntos de datos y agregar más características complejas. Se espera que los CRF también puedan ser aplicados a otras tareas de clasificación no tabular, que involucren contenido con varios formatos y diseños. En general, 
los CRF pueden ayudar en la construcción de sistemas de extracción de información genérica.

\section{Referencias}

[1] M. Yakout, K. Ganjam, K. Chakrabarti, and S. Chaudhuri, "Infogather: Entity augmentation and attribute discovery by holistic matching with web tables," in Proceedings of the 2012 ACM SIGMOD International Conference on Management of Data, ser. SIGMOD '12. New York, NY, USA: Association for Computing Machinery, 2012, pp. 97-108. [Online]. Available: https://doi.org/10.1145/2213836.2213848

[2] M. J. Cafarella, A. Halevy, D. Z. Wang, E. Wu, and Y. Zhang, "Webtables: Exploring the power of tables on the web," Proc. VLDB Endow., vol. 1, no. 1 , pp. 538-549, Aug. 2008. [Online]. Available: https://doi.org/10.14778/1453856.1453916

[3] E. Koci, M. Thiele, O. Romero, and W. Lehner, "Table identification and reconstruction in spreadsheets," in Advanced Information Systems Engineering, E. Dubois and K. Pohl, Eds. Cham: Springer International Publishing, 2017, pp. 527541.

[4] P. Venetis, A. Halevy, J. Madhavan, M. Paşca, W. Shen, F. Wu, G. Miao, and C. Wu, "Recovering semantics of tables on the web," Proc. VLDB Endow., vol. 4, no. 9, pp. 528-538, Jun. 2011. [Online]. Available: https://doi.org/10.14778/2002938.2002939

[5] G. Limaye, S. Sarawagi, and S. Chakrabarti, "Annotating and searching web tables using entities, types and relationships," Proc. $V L D B$ Endow., vol. 3, no. 1-2, pp. 1338-1347, Sep. 2010. [Online]. Available: https://doi.org/10.14778/1920841.1921005

[6] T. F. Varish Mulwad and A. Joshi, "Generating Linked Data by Inferring the Semantics of Tables," in Proceedings of the First International Workshop on Searching and Integrating New Web Data Sources, September 2011, co-located with VLDB 2011. [Online]. Available: https://bit.ly/3p8s1q0

[7] A. S. Corrêa and P.-O. Zander, "Unleashing tabular content to open data: A survey on pdf table extraction methods and tools," in Proceedings of the 18th Annual International Conference on Digital Government Research, ser. dg.o '17. New York, NY, USA: Association for Computing Machinery, 2017, pp. 54-63. [Online]. Available: https://doi.org/10.1145/3085228.3085278
[8] B. Yildiz, K. Kaiser, and S. Miksch, "pdf2table: A method to extract table information from pdf files." [Online]. Available: https://bit.ly/3k2ejBa

[9] Y. Liu, P. Mitra, and C. L. Giles, "Identifying table boundaries in digital documents via sparse line detection," in CIKM '08, 2008. [Online]. Available: https://bit.ly/369nWcm

[10] T. Kieninger, "Table structure recognition based on robust block segmentation," 1998, pp. 22-32. [Online]. Available: https://bit.ly/38k4YT9

[11] M. Zhang and K. Chakrabarti, "Infogather+: Semantic matching and annotation of numeric and time-varying attributes in web tables," in Proceedings of the 2013 ACM SIGMOD International Conference on Management of Data, ser. SIGMOD '13. New York, NY, USA: Association for Computing Machinery, 2013, pp. 145-156. [Online]. Available: https://doi.org/10.1145/2463676.2465276

[12] Z. Zhang, "Towards efficient and effective semantic table interpretation," in The Semantic $W e b$ - ISWC 2014, P. Mika, T. Tudorache, A. Bernstein, C. Welty, C. Knoblock, D. Vrandečić, P. Groth, N. Noy, K. Janowicz, and C. Goble, Eds. Cham: Springer International Publishing, 2014, pp. 487-502. [Online]. Available: https://doi.org/10.1007/978-3-319-11964-9_31

[13] H. Masuda and S. Tsukamoto, "Recognition of html table structure," 2004. [Online]. Available: https://bit.ly/3p8xL2Q

[14] J. Fang, P. Mitra, Z. Tang, and C. L. Giles, "Table header detection and classification," in $A A A I$, 2012. [Online]. Available: https://bit.ly/2IcT3vy

[15] D. Pinto, A. McCallum, X. Wei, and W. B. Croft, "Table extraction using conditional random fields," in Proceedings of the 26th Annual International ACM SIGIR Conference on Research and Development in Informaion Retrieval, ser. SIGIR '03. New York, NY, USA: Association for Computing Machinery, 2003, pp. 235-242. [Online]. Available: https://doi.org/10.1145/860435.860479

[16] I. A. Doush and E. Pontelli, "Detecting and recognizing tables in spreadsheets," in Proceedings of the 9th IAPR International Workshop on Document Analysis Systems, ser. DAS '10. New York, NY, USA: Association for Computing Machinery, 2010, pp. 471-478. [Online]. Available: https://doi.org/10.1145/1815330.1815391

[17] E. Koci, M. Thiele, W. Lehner, and O. Romero, "Table recognition in spreadsheets via a graph 
representation," in 2018 13th IAPR International Workshop on Document Analysis Systems (DAS), 2018, pp. 139-144. [Online]. Available: https://doi.org/10.1109/DAS.2018.48

[18] J. D. Lafferty, A. McCallum, and F. C. N. Pereira, "Conditional random fields: Probabilistic models for segmenting and labeling sequence data," in Proceedings of the Eighteenth International Conference on Machine Learning, ser. ICML '01. San Francisco, CA, USA: Morgan Kaufmann
Publishers Inc., 2001, pp. 282-289. [Online]. Available: https://bit.ly/3lbW1yE

[19] J. L. Solé, Book review: Pattern recognition and machine learning. Cristopher M. Bishop. Information Science and Statistics. Springer, 2007. [Online]. Available: https://bit.ly/317doRq

[20] M. D. Adelfio and H. Samet, "Schema extraction for tabular data on the web," Proc. $V L D B$ Endow., vol. 6, no. 6, pp. 421-432, Apr. 2013. [Online]. Available: https://doi.org/10.14778/2536336.2536343 


\title{
ANÁlisis DE LA EFICIENCIA DE UN DISCO DE FRENO CONVENCIONAL VENTILADO CON RESPECTO A UN DISCO HIPERVENTILADO MEDIANTE MECANIZADO ANALisys of THE EFFICIENCY OF A Conventional Ventilated Brake Disc Compared to a Hyperventilated Disc By MACHINING
}

\author{
Vicente Rojas ${ }^{1, *}$, Johnny Pancha ${ }^{2}$, Vicente Romero $^{3}$, Jorge Lema ${ }^{3}$ \\ Recibido: 06-04-2020, Revisado: 22-09-2020, Aprobado tras revisión: 17-10-2020
}

\section{Resumen}

Este estudio tiene como finalidad rediseñar un disco de freno ventilado a un disco hiperventilado para comparar las diferencias de temperatura y distancias de frenado, para ello se instaló un sistema de monitoreo en el vehículo, que consistió en la implementación de dos sensores de temperatura ubicados cerca de cada disco y una tarjeta de adquisición de datos. Con la implementación de los discos hiperventilados se pudo disminuir la temperatura generada por la fricción del frenado. Se realizó el monitoreo de valores de temperaturas que se producen entre los discos al momento de frenar. Para obtener los valores de temperaturas de los discos de frenos, se realizaron pruebas de ruta con diferentes tipos de discos: discos ventilados en las dos ruedas frontales, discos hiperventilados en las dos ruedas frontales y discos mixtos. En los discos mixtos van un disco ventilado en rueda delantera derecha y uno hiperventilado en rueda delantera izquierda.

\section{Abstract}

This study is to redesign a ventilated brake disc to a hyperventilated disc to compare the differences in temperature and brake distances, for this a monitoring system was installed in the vehicle which consisted of the implementation of two sensors of temperature located near each disk and a data acquisition card. With the implementation of hyperventilated discs, the temperature generated by braking friction could be reduced. The temperature values that occur between the discs when braking were monitored. To obtain the temperature values of the brake discs, road tests were carried out with different types of discs: ventilated discs on the two front wheels, hyperventilated discs on the two front wheels and mixed discs. In the mixed discs there is a ventilated disc on the right front wheel and a hyperventilated disc on the left front wheel.

\footnotetext{
1,*Universidad Politécnica Salesiana, Ecuador. Autor para correspondencia ${ }^{\star}$ erojas@ups.edu.ec.

(D) http://orcid.org/0000-0001-5658-3055.

${ }^{2}$ Escuela Superior Politécnica de Chimborazo, Ecuador. (D) http://orcid.org/0000-0001-7320-2154.

${ }^{3}$ Universidad Castilla La Mancha, España. (D) http://orcid.org/0000-0003-2317-7071

${ }^{4}$ Universidad Tecnológica Indoamérica, Ecuador. (D) http://orcid.org/0000-0002-1515-4526
}

Forma sugerida de citación: Rojas, V.; Pancha, J.; Romero, V. y Lema, J. (2021). «Análisis de la eficiencia de un disco de freno convencional ventilado con respecto a un disco hiperventilado mediante mecanizado». INGENIUS. N. ${ }^{\circ} 25$, (enero-junio). pp. 62-69. DOI: https://doi.org/10.17163/ings.n25.2020.06. 
Mediante visualización de datos en tiempo en los discos de freno se pudo concluir que los hiperventilados poseen una mejor disipación de calor ya que presentan una mejor ventilación. A partir de todos los resultados de las pruebas de rutas obtenidas, se pudo visualizar el comportamiento de temperatura en los discos al momento de frenar y se evidenció que los discos hiperventilados tienden a calentarse menos que los normales, llevando con esto a disminuir el tiempo y distancia de frenado.

Palabras clave: disco de freno, disco ventilado, disco hiperventilado, temperatura de disco
By displaying time data on the brake discs, it was possible to conclude that hyperventilated discs have better heat dissipation since they present better ventilation. From all the results of the route tests obtained, it was possible to visualize the temperature behavior in the discs at the moment of braking and it was evidenced that hyperventilated discs tend to heat up less than normal discs, thus leading to a decrease in time and stopping distance.

Keywords: Brake Disc, Ventilated Disc, Hyperventilated Disc, Disc Temperature. 


\section{Introducción}

Un vehículo al circular varía continuamente su estado, acelera, frena o gira. Estos fenómenos son producidos por un gran número de fuerzas y su suma se denomina dinámica del vehículo. Si la suma de todas las fuerzas es cero, significa que está en reposo. Si es diferente de cero, estará en movimiento. A su vez, todas estas fuerzas varían en función de una magnitud física denominada aceleración, responsable de modificar la velocidad y dirección de cualquier objeto. Por ejemplo, el hecho de acelerar el coche corresponde a una aceleración positiva y el caso de frenar a una aceleración negativa [1].

El sistema de frenos es, sin duda, el componente más importante para la seguridad vial del automóvil, ya que de este depende la detención total o parcial del vehículo y, en consecuencia, la integridad de sus pasajeros. Generalmente el $70 \%$ de la energía cinética producida en el movimiento es absorbida por los frenos de disco delanteros y el restante por el freno trasero, que suele ser de tambor. Estos sistemas se fundamentan en la fricción para detener el movimiento del vehículo, teniendo como principio la presión hidráulica que empuja las pastillas de freno contra el disco de hierro fundido. En consecuencia, el comportamiento que produce este tipo de dispositivos, a través de la energía cinética, es crear un calor considerablemente alto durante el frenado, aumentando la temperatura por fricción; este calor se disipa rápidamente con el aire circundante por medio del fenómeno de convección (transferencia de calor que se produce entre masas a diferente temperatura) [2].

La determinación de las características geométricas de los discos depende de la capacidad de carga y el funcionamiento, los cuales son factores importantes en la fase de diseño inicial. En la mayoría de los casos, el diseño de los discos debe evitar el sobrecalentamiento que surge entre el freno y la pastilla por el efecto de la fricción, seleccionando de forma adecuada las propiedades físicas, mecánicas y químicas que se producen debido a que en algunas ocasiones los tipos de materiales no se comportan correctamente y tienen efectos negativos sobre la eficacia del proceso de frenado. En la etapa del diseño mecánico en frenos de discos ventilados, es muy importante analizar el comportamiento de los termofluidos asociados (aire circundante), es decir, observar las características y operación de los fluidos sobre la superficie del disco, siempre garantizando la efectividad del proceso de frenado y la disipación de calor por la superficie y los canales de ventilación [3].

Durante el proceso de frenado, el calor producido por la fricción entre las pastillas de freno y el disco no es disipado rápidamente, esto depende de la geometría y del material de fabricación. Por consiguiente, en el freno de disco cuando se provoca un frenado muy fuerte, se pueden acumular grandes cantidades de calor en un corto tiempo, produciéndose altos gradientes de temperatura. En estas condiciones, la funcionalidad y la seguridad del sistema de freno pueden estar comprometidos [4]. Los frenos de disco han sido muy utilizados en los vehículos por su correcto funcionamiento debido a que absorben $70 \%$ de la energía cinética producida en el movimiento, la cual ha sido su principal ventaja con respecto al freno de tipo tambor. Cuando se producen frenados repetitivos en los discos de freno aumenta la temperatura debido a la fricción que existe con la pastilla, generando gradientes de temperatura. Este calor se disipa rápidamente con el aire circundante por medio del fenómeno de convección (transferencia de calor que se produce entre masas a diferente temperatura). Las altas temperaturas pueden causar la vaporización del líquido de frenos, desgaste del freno, fallas en los cojinetes, grietas térmicas (fading) y vibraciones. Por tal motivo, en muchas ocasiones se reduce el rendimiento del sistema; por lo anterior, resulta de gran importancia predecir el comportamiento de los tipos de convección presentes en la disipación del calor al medioambiente con la finalidad de evaluar su eficiencia teniendo en cuenta su diseño y geometría inicial [5].

El sistema de frenos es un sistema primordial de seguridad para evitar cualquier accidente durante el manejo de todo tipo de vehículo. El problema presente en estos sistemas es debido al sobrecalentamiento en los componentes del sistema, por ende, es indispensable el seguimiento del compartimento de la temperatura en los discos de freno [6]. Uno de los factores indispensables para análisis y estudio son las alteraciones de temperatura al momento de accionar los frenos. En los últimos tiempos, con el crecimiento tecnológico, se están implementando discos ventilados, los mismos que ayudan a reducir el sobrecalentamiento y evitar en gran medida los accidentes de tránsito [7].

El uso de estos sistemas modernos se ve aplicado solo en vehículos de alta gama, debido a su costo superior con respecto a un sistema convencional [8]. La implementación de discos ventilados en el presente trabajo tiene como objetivo mejorar la disipación de calor producido por la acción del frenado, a un costo más bajo y accesible.

\section{Materiales y métodos}

\subsection{Diseño del disco}

Para el desarrollo del diseño del disco de freno hiperventilado se utilizó el software de diseño Solidworks [9], y partimos de un disco de freno normal de un vehículo Chevrolet Dmax 4x4.

Sobre el plano inicial se moldearon los ejes principales como se puede observar en la Figura 1, sobre los cuales irán orificios y ranuras, partes claves para el 
desarrollo del proyecto, fueron trazados tomando en cuenta la geometría y forma del disco de frenos sobre el que se trabajará posteriormente.

Las dimensiones de orificios, ranuras, profundidades y distancias, detalladas en la Figura 2, fueron elegidas a criterio de los autores, las cuales irán centradas sobre los ejes ya descritos anteriormente.

Tomando las dimensiones y detalles del disco de frenos se realizó un modelado inicial detallado en la Figura 3 con objetivo de visualizar las formas y geometrías del mismo en el software.

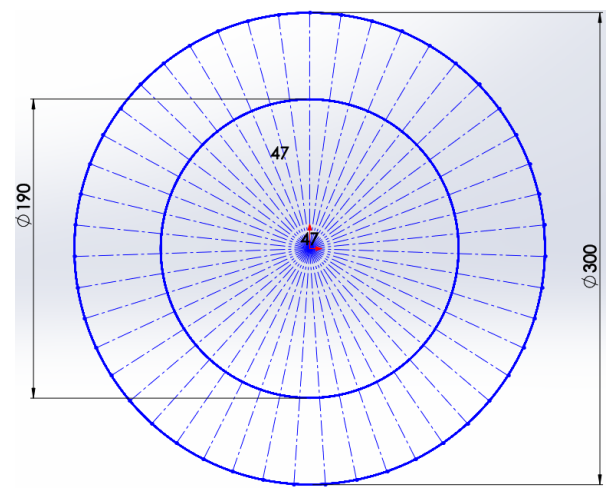

Figura 1. Delineamiento de ejes geométricos principales

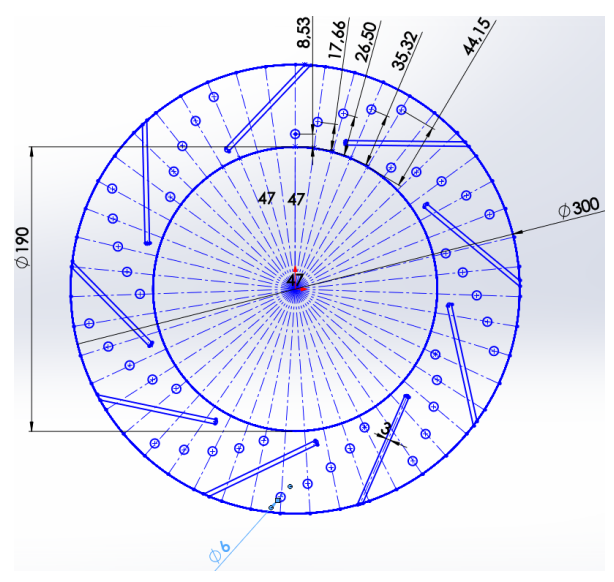

Figura 2. Trazo de orificios y ranuras

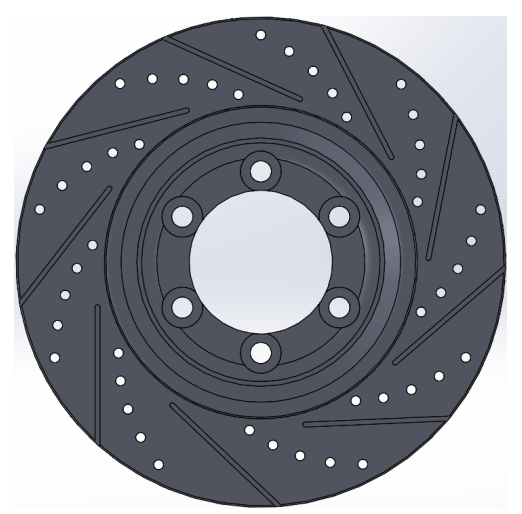

Figura 3. Modelado de disco ventilado [3]

\subsection{Mecanizado del disco de freno}

En esta parte se mecanizó las modificaciones al disco normal de frenos previamente diseñadas con el software. Todos los datos geométricos fueron enviados hacia la máquina herramienta $\mathrm{CNC}$, en este caso la fresadora, con la cual se pudo conseguir mayor precisión, facilitando el trabajo. Todos los planos que se realizaron previamente en Solidworks se tuvieron que exportar al software con el cual trabaja la fresadora CNC, Figura 4, es decir, el Mastercam, un software bastante práctico y, además, compatible con Solidworks [10].

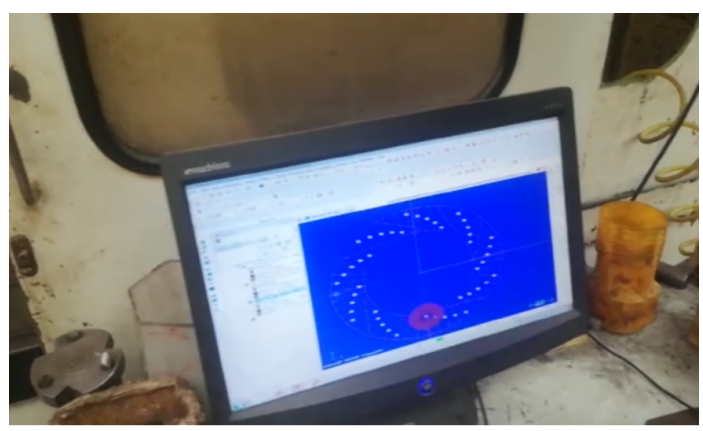

Figura 4. Asignación de puntos a perforar en el disco

Con las coordenadas de trabajo fijas se procedió a perforar los orificios que ventilarán el disco de freno, siendo estos agujeros totalmente pasantes al disco. En la Figura 5 se muestra la perforación y la ejecución del código G.

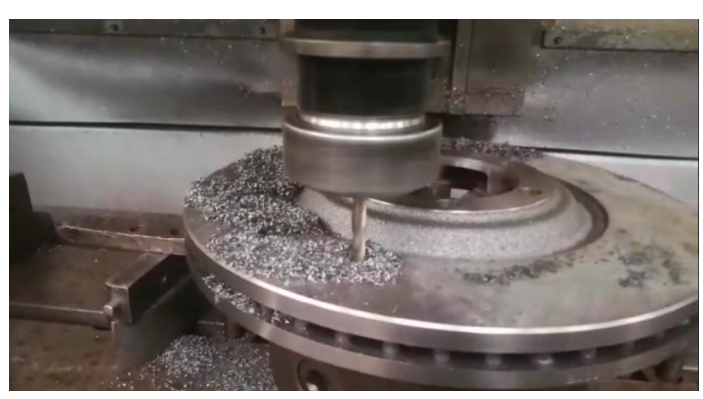

Figura 5. Perforación al disco de freno

Luego de la perforación se ejecutó el software para desbastar las ranuras que se emplean para ventilación y evacuación de virutas en el disco. La profundidad y espesor de las ranuras se determinó a discreción de los autores. Se cuidó de no comprometer los espesores del disco; se optó por una profundidad de $2.5 \mathrm{~mm}$ y espesor de $3 \mathrm{~mm}$, como se observa en la Figura 6. 


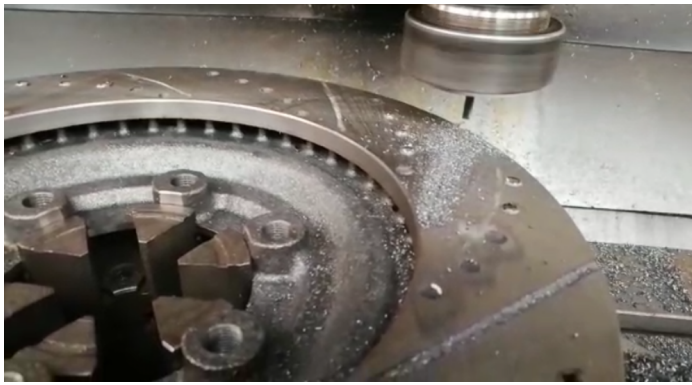

Figura 6. Desbaste de ranuras de ventilación

\subsection{Implementación de hardware y sensores}

La cantidad de hardware disponible en el mercado actual es considerable, por lo que se puede obtener un nivel de flexibilidad muy importante a la hora de buscar una configuración adecuada [11].

Seleccionando a la tarjeta Arduino como procesador de monitoreo de temperatura y sensores (MLX90614) para la adquisición de datos de temperatura de los discos de freno, como se muestra en la Figura 7 y Figura 8, respectivamente.

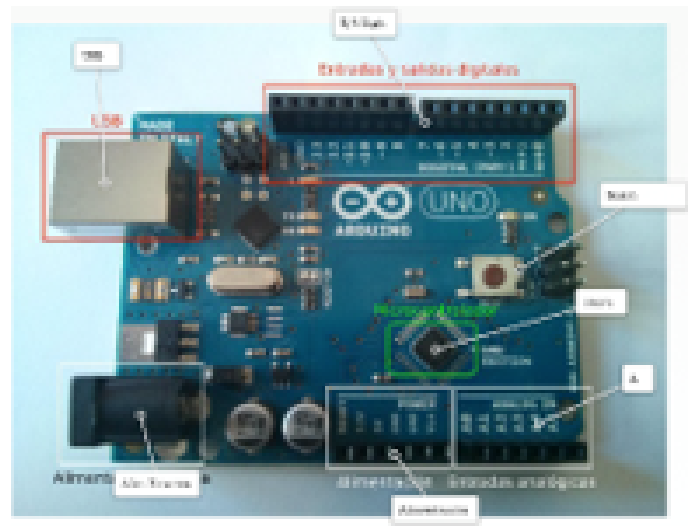

Figura 7. Tarjeta Arduino

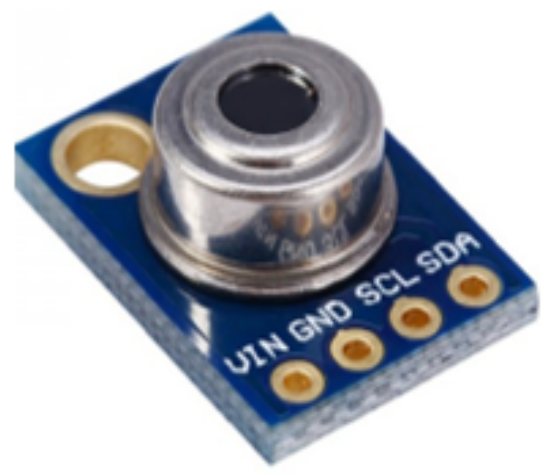

Figura 8. Sensor de temperatura por infrarrojo

\subsection{Programación del software de adquisición de datos}

El sistema de monitoreo de temperatura de los discos de freno está compuesto por sensores de temperatura, placa electrónica Arduino, cable serial de comunicaciones y un ordenador portátil. Para la programación y asignación de parámetros se utilizó el software LabVIEW 2017 [12].

Las pantallas gráficas fueron modificadas y renombradas de acuerdo con el requerimiento del proyecto sistema de monitoreo de temperatura, teniendo como resultado la pantalla gráfica (Figura 9).

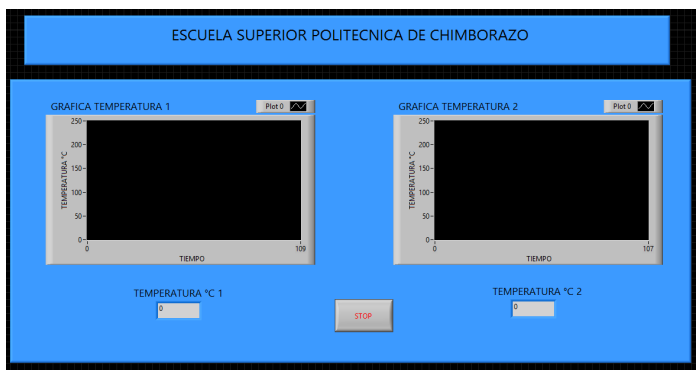

Figura 9. Pantalla gráfica de temperatura

\subsection{Implementación de discos, sensores y sistema de adquisición de datos}

Para la correcta instalación de los discos de freno se requiere: herramienta de retracción de pistón, reloj comparador, llave dinamométrica y otras herramientas como se aprecian en la Figura 10.

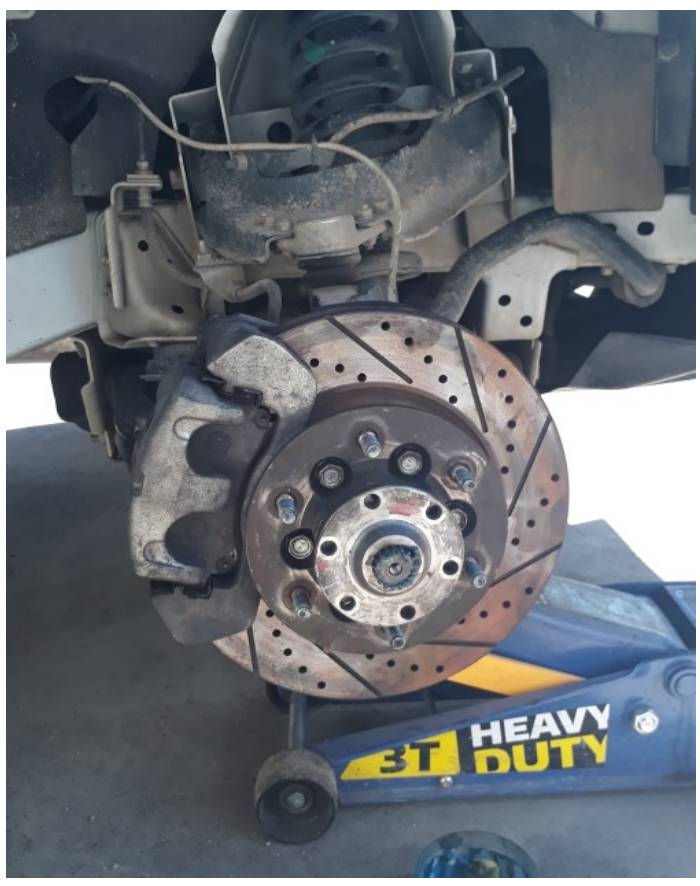

Figura 10. Implementación del disco ventilado 
Rojas. et al/ Análisis de la eficiencia de un disco de freno convencional ventilado con respecto a un disco

Se instaló el sistema de adquisición de datos para el monitoreo de la temperatura de los discos de freno, utilizando la tarjeta Arduino y sensores de temperatura a distancia (MLX90614).

Para el fácil acceso y manipulación se procedió con la ubicación de la tarjeta Arduino dentro del habitáculo del automóvil (Figura 11).

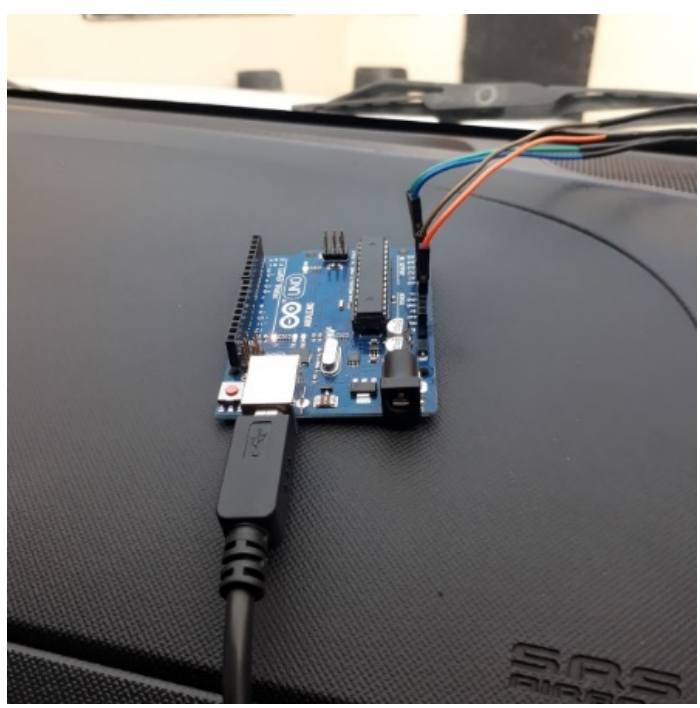

Figura 11. Ubicación del Arduino

Los sensores de temperatura son instalados muy próximos a los discos ventilados, se ubicó en lámina protectora del disco de freno, fue utilizada como base de soporte para el montaje de los sensores como se aprecia en la Figura 12.

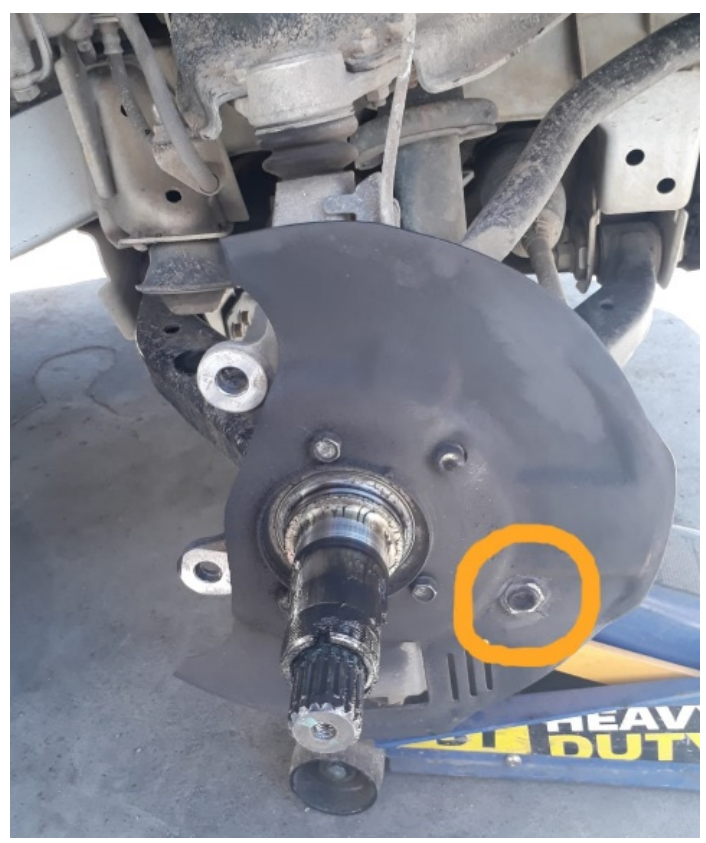

Figura 12. Ubicación de los sensores de temperatura

\section{Resultados y discusión}

Inicialmente se realizó el monitoreo de temperatura con discos de freno originales de Dmax 4x4, en un circuito urbano e interurbano.

Posteriormente se hizo la adquisición de datos de temperatura con discos de freno ventilados rediseñados; la prueba se desarrolló en la misma ruta seleccionada anteriormente.

Finalmente, se monitorearon los datos de temperaturas, instalando un disco original en la rueda delantera izquierda y con disco hiperventilado en la rueda delantera derecha. En este apartado se realizó la adquisición de datos de temperatura con discos normales, a diferentes velocidades del vehículo, en la ruta seleccionada (Tambo-Cañar),

En la Figura 13 se detallan datos de temperatura monitoreada con discos de frenos hiperventilados obteniendo un rango de temperatura de trabajo que oscila entre $80{ }^{\circ} \mathrm{C}$ y $100{ }^{\circ} \mathrm{C}$.

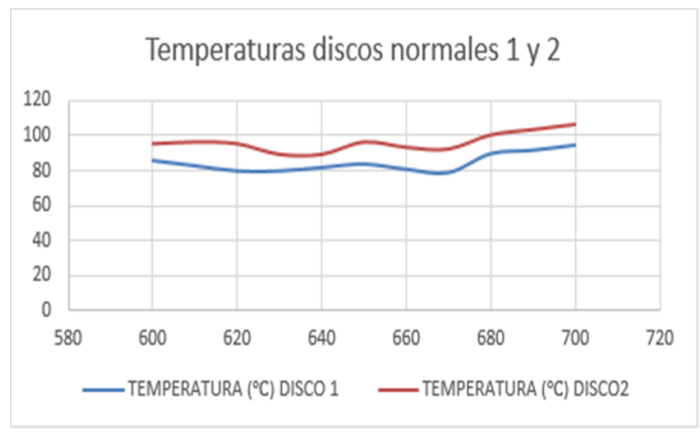

Figura 13. Temperatura de discos ventilados

Para la adquisición de datos de temperatura con los discos originales, las pruebas se realizan en la misma ruta seleccionada (Tambo-Cañar), los resultados obtenidos con discos ventilados se detallan en la Figura 14. Obteniéndose un rango de temperatura de trabajo que oscila entre 90 y $130{ }^{\circ} \mathrm{C}$.

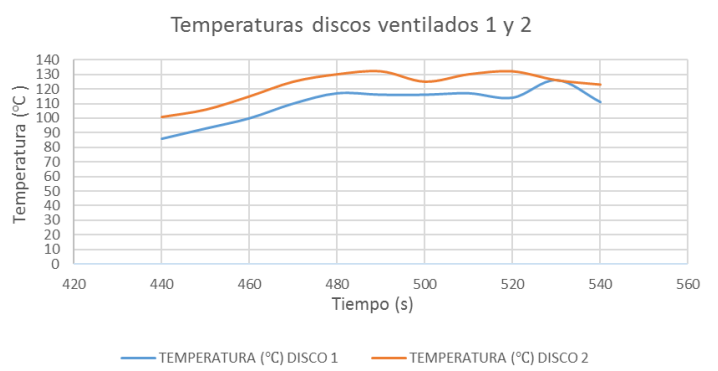

Figura 14. Temperatura de discos hiperventilados

\section{Prueba de distancia de frenado con discos ori- ginales}

El vehículo fue puesto a prueba con discos normales de fabricación con las condiciones mostradas en la Tabla 1. 
Tabla 1. Condiciones y resultados de prueba para distancia de frenado con discos convencionales

\begin{tabular}{cc}
\hline Velocidad inicial $(\mathrm{km} / \mathrm{h})$ & $\mathbf{1 0 0}$ \\
\hline Tiempo inicial $(\mathrm{s})$ & 1637 \\
Temperatura inicial $\left({ }^{\circ} \mathrm{C}\right)$ & 64 \\
Velocidad final $(\mathrm{km} / \mathrm{h})$ & 0 \\
Tiempo final $(\mathrm{s})$ & 1641 \\
Temperatura final $\left({ }^{\circ} \mathrm{C}\right)$ & 82 \\
Distancia de frenado $(\mathrm{m})$ & 37,5 \\
Tiempo de frenado $(\mathrm{s})$ & 4 \\
\hline
\end{tabular}

\section{Prueba de frenado con discos hiperventilados}

En el vehículo de pruebas se colocaron los discos hiperventilados y se realizaron las pruebas con las condiciones de la Tabla 2.

Tabla 2. Condiciones y resultados de prueba para distancia de frenado con discos hiperventilados

\begin{tabular}{cc}
\hline Velocidad inicial $(\mathrm{km} / \mathrm{h})$ & $\mathbf{1 0 0}$ \\
\hline Tiempo inicial $(\mathrm{s})$ & 178 \\
Temperatura inicial $\left({ }^{\circ} \mathrm{C}\right)$ & 39 \\
Velocidad final $(\mathrm{km} / \mathrm{h})$ & 0 \\
Tiempo final $(\mathrm{s})$ & 181 \\
Temperatura final $\left({ }^{\circ} \mathrm{C}\right)$ & 55 \\
Distancia de frenado $(\mathrm{m})$ & 25 \\
Tiempo de frenado $(\mathrm{s})$ & 3 \\
\hline
\end{tabular}

Por último, se comparan las dos pruebas de distancia de frenado anteriores con discos originales y los hiperventilados y se muestran sus resultados en la Figura 15.

Para realizar las gráficas comparativas se descartaron los tiempos de inicio y final ya que estos pertenecen al programa en el periodo que permanece ejecutándose, por lo que no son valores significativos para la comparativa de la distancia de frenado.

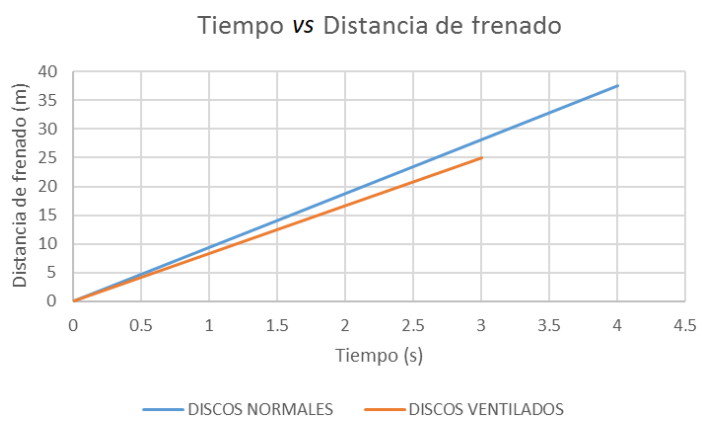

Figura 15. Comparativa de las distancias de frenado de los discos originales e hiperventilados

Se obtuvo una distancia de frenado de $37 \mathrm{~m}$ con los discos originales y una distancia de frenado de $25 \mathrm{~m}$ con el disco hiperventilado.
Tomando en cuenta el gasto computacional y el tiempo de operación de mecanizado, el costo del rediseño con respecto al disco original tiene un incremento del $30 \%$ del valor del mercado.

\section{Conclusiones}

Gracias al software Solidworks se rediseñó de una manera más fácil y precisa, con lo que posteriormente se pudo mecanizar el disco con la ayuda de una fresadora CNC para obtener resultados de rediseño exactos y fiables.

El sistema de monitoreo de temperatura de los discos de freno permitió visualizar, en tiempo real, los datos del calor producido por las cargas térmicas al momento del frenado del vehículo.

Los discos de frenos ventilados disiparon el calor de una forma más eficaz que los normales, consiguiendo mejor eficiencia, menor tiempo y distancia de frenado y mayor seguridad durante la conducción.

El costo del rediseño tiene un incremento del $30 \%$ con respecto al disco original, justificándose este valor con los datos de tiempo y distancia de frenado obtenido en este estudio.

Para estudios futuros se puede analizar la concentración de esfuerzos axiales en el disco modificado, con el fin de comprobar su durabilidad y tiempo de vida útil.

\section{Referencias}

[1] L. G. Ayala Ayala and J. P. Vallejo Orbe, "Adaptación de un sistema de frenos ABS a un vehículo Fiat, para mejorar la seguridad del frenado," 2013. [Online]. Available: https://bit.ly/318an3l

[2] R. García-León, M. Acosta, and E. Solano, "Análisis del comportamiento de los frenos de disco de los vehículos a partir de la aceleración del proceso de corrosión," Tecnura, vol. 19, 07 2015. [Online]. Available: http://doi.org/10. 14483/udistrital.jour.tecnura.2015.3.a04

[3] R. A. García-León, R. D. Echavez Díaz, and E. Flórez Solano, "Análisis termodinámico de un disco de freno automotriz con pilares de ventilación tipo NACA 66-209," INGE CUC, vol. 14, no. 2, pp. 9-18, 2018. [Online]. Available: https://doi.org/10.17981/ingecuc.14.2.2018.01

[4] R. A. García León, "Estudio térmico en tres frenos de disco ventilados, utilizando el análisis de elementos finitos," $D Y N A$, vol. 84, no. 200, pp. 15-30, 2017. [Online]. Available: http://dx.doi.org/10.15446/dyna.v84n200.55663 
[5] R. A. García León and E. N. Flórez Solano, "Estudio analítico de la transferencia de calor por convección que afectan a los frenos de disco ventilados," Tecnura, vol. 20, pp. 15-30, mar. 2017. [Online]. Available: https://doi.org/10.14483/22487638.11676

[6] E. Águeda Casado, T. Gómez Morales, and J. Martín Navarro, Sistemas de transmisión de fuerzas y trenes de rodaje, ser. Ciclos formativos. Ediciones Paraninfo, 2012. [Online]. Available: https://bit.ly/2IcXcj6

[7] Aficionados a la Mecánica. (2020.) Ingeniería automotriz. [Online]. Available: https: //bit.ly/3k7EzKC

[8] Y. Carranza Sánchez and R. Beltrán, "Transferencia de calor de estado inestable en forros para frenos," 07 2003. [Online]. Available: https://bit.ly/354APFf

[9] F. Kreith, M. S. Bohn, and R. M. Manglik, Principios de Transferencia de Calor. Cengage Learning Editores, 2012. [Online]. Available: https://bit.ly/36dOPfh

[10] F. P. Incropera and D. P. DeWitt, Fundamentos de transferencia de calor. Pearson Educación, 1999. [Online]. Available: https://bit.ly/3ezzLw8

[11] Kashima University. (2016) Curso ingeniería automotriz. [Online]. Available: https: //bit.ly/3mXaTRW

[12] D. Wenner, Manual práctico del cuidado y reparación del automóvil. México Continental, 1984. [Online]. Available: https://bit.ly/32jIr4P 


\title{
EVALUACIÓN DE UN RADAR FMCW COMO HERRAMIENTA DIDÁCTICA EN LAS CARRERAS DE INGENIERÍA AUTOMOTRIZ Y TELECOMUNICACIONES \\ EVALUATION OF A FMCW RADAR AS A TEACHING TOOL IN THE AUTOMOTIVE AND TELECOMMUNICATIONS ENGINEERING CAREERS
}

\author{
Pablo J. Mavares F.,** \\ Recibido: 10-05-2020, Revisado: 22-09-2020, Aprobado tras revisión: 17-10-2020
}

\section{Resumen}

En las últimas décadas los sistemas avanzados de asistencia al conductor (ADAS) han evolucionado hasta estar disponibles en gran parte de los vehículos fabricados hoy en día; mantener actualizada la enseñanza en esta área es de vital importancia. Este artículo presenta un radar de onda continua modulado en frecuencia que trabaja en la banda de $24 \mathrm{GHz}$ ISM. El propósito es evaluar su desempeño e idoneidad para usarse como herramienta didáctica en la enseñanza en las carreras de Ingeniería Automotriz y de Telecomunicaciones con énfasis en las asignaturas de Matemáticas y Telecomunicaciones con escenarios factibles de encontrar en los laboratorios universitarios. Para ello se describe el escenario de medición, así como el hardware, el firmware y un algoritmo genérico implementado en MATLAB basado en transformadas rápidas de Fourier para obtener mapas RangeDoppler que permiten junto con el algoritmo CFAR mejorar la detección de objetos al comparar con la detección a partir de un nivel fijo. Se presentan resultados que demuestran que la exactitud y precisión del radar se encuentran dentro de los parámetros para un radar de corto alcance para vehículos, encontrándose, además, una herramienta con gran potencial didáctico, con la cual los estudiantes pueden comprender las aplicaciones que hoy tienen las matemáticas en el ámbito de las telecomunicaciones, especialmente en radares que sirven a sistemas ADAS.

Palabras clave: radar FMCW, mapa RangeDoppler, CFAR, herramienta didáctica

\section{Abstract}

In recent decades, advanced driver-assistance systems (ADAS) have evolved to be available in much of the vehicles manufactured today; it is very im-portant to keep teaching in this area up-to-date. This paper presents a frequency modulated continuous wave radar that works in the $24 \mathrm{GHz}$ ISM band. The purpose of this work is to evaluate its performance and suitability to be used as a didactic tool in teach-ing in the automotive and telecommunications engi-neering careers with an emphasis on the mathematics and telecommunications subjects, under scenarios feasible to be found in university labs. For this pur-pose, the measurement scenario is described, as well as the hardware, firmware and a generic algorithm implemented in MATLAB based on fast Fourier transforms to obtain Range-Doppler maps that allow, in conjunction with the CFAR algorithm, to improve detection of objects when compared to the detection from a fixed level. The results presented demonstrate that the accuracy and precision of the radar are within the parameters for a short-range radar for vehicles, also finding a tool with great didactic potential with which students can understand today's applications of mathematics in the field of telecommunications, especially in radars that serve ADAS systems.

Keywords: FMCW radar, Range-Doppler map, CFAR, didactic tool.

\footnotetext{
${ }^{1}$ Departamento de Sistemas de Automatización, Instituto Tecnológico El Pacífico - Ecuador.

Autor para correspondencia: pmavares@tecnologicopacifico.edu.ec (D) http://orcid.org/0000-0001-7342-6262
}

Forma sugerida de citación: Mavares, P. J. (2021). «Evaluación de un radar FMCW como herramienta didáctica en las carreras de Ingeniería Automotriz y Telecomunicaciones». Ingenius. N. ${ }^{\circ}$ 25, (enero-junio). pp. 70-80. DOI: https://doi.org/10.17163/ings.n25.2021.07. 


\section{Introducción}

El radar ha sido empleado tradicionalmente en la industria militar y aeronáutica durante varios años, principalmente debido a la elevada complejidad y el alto costo que ha llevado su uso. No obstante, con el evolucionar de la electrónica y los circuitos integrados (CI) cada día es más común encontrar radares con especificaciones adecuadas para otras tareas, dentro de las que se puede mencionar la industria automotriz [1]. Particularmente en los últimos años se han empleado en los sistemas de ayuda avanzada al conductor (ADAS por sus siglas en inglés) [2]. Para ello, los sistemas de radar miden de forma directa o indirecta la posición, velocidad e incluso la aceleración de una gran variedad de objetos como otros vehículos, peatones o ciclistas gracias a la ejecución de diferentes algoritmos [3].

Aunque actualmente también se emplean sistemas de videocámaras, ultrasonido, etc. Los radares tienen la ventaja de ser poco o nulamente afectados por las condiciones ambientales como la temperatura, iluminación, polvo, etc. Es por ello por lo que pueden funcionar de manera individual o en conjunto con otras tecnologías para garantizar la elevada exactitud, precisión, confiabilidad y adaptabilidad que requieren los sistemas ADAS [4].

Además de la detección de objetos, los radares tienen otras aplicaciones como la identificación de las condiciones físicas, particularmente el coeficiente de fricción de una carretera. Algo que puede ser empleado para emitir alertas o tomar acciones para evitar accidentes, especialmente bajo condiciones adversas como lluvia o hielo [5], [6].

También, existe la posibilidad de usar transpondedores o tags para el envío de información acerca de usuarios en la carretera como otros vehículos o peatones y también información acerca de las condiciones de conducción de forma tal que se puedan evitar accidentes o infracciones de tránsito [5].

Los radares de onda continua modulados en frecuencia (FMCW por sus siglas en inglés) se han aplicado satisfactoriamente para la medición de la posición y velocidad de objetos desde hace bastante tiempo [7].

Los radares FMCW trabajan básicamente en frecuencias de 24 y $77 \mathrm{GHz}$ en la industria automotriz. Para un alcance corto de hasta unas decenas de metros es posible usar un radar de $24 \mathrm{GHz}$, mientras que para alcance largo de unos $250 \mathrm{~m}$ se emplea uno a $77 \mathrm{GHz}$ [3], [5].

Diversas investigaciones se han realizado desde una perspectiva técnica para evaluar diversos sistemas de radar FMCW tanto a 24 como $77 \mathrm{GHz}$ para su uso en la industria automotriz [6-12]. No obstante, en este trabajo se plantea el uso de estos radares desde una perspectiva diferente, que aparte de la evaluación del desempeño sea enfocada en su potencialidad en el uso como herramienta didáctica que permita im- pulsar la comprensión de conceptos que en muchas ocasiones pueden ser bastantes complejos de entender como es la transformada rápida de Fourier (FFT), los radares y sus aplicaciones en los campos de las telecomunicaciones y la industria automotriz. Por ello se propone el empleo de tarjetas de evaluación de radares considerando los beneficios en el desarrollo de competencias específicas y transversales en estudiantes.

\subsection{Parámetros importantes en un radar FMCW}

Para procesar y obtener la posición, velocidad y ángulo es necesario considerar diversas ecuaciones, cuya derivación puede encontrarse en [4], [13]. Estas ecuaciones se derivan a partir de la siguiente idea general del funcionamiento de un radar FMCW. El radar transmite una señal con frecuencia variable en forma lineal (TX) con cierto ancho de banda, esta señal es reflejada por un cuerpo que se encuentre en el espacio de radiación de la antena y llega como una señal a la antena receptora (RX) con un tiempo de retardo proporcional a la distancia del cuerpo. Estas señales son mezcladas y se obtiene una señal de frecuencia intermedia (IF), cuya frecuencia es proporcional a la distancia del cuerpo (Figura 1). Por lo tanto, cuerpos a diferentes distancias generarán diferentes frecuencias de señal IF. Por otra parte, pequeñas diferencias de posición generarán diferentes fases de las señales IF lo que permite determinar la velocidad de los cuerpos [2], [4], [14].

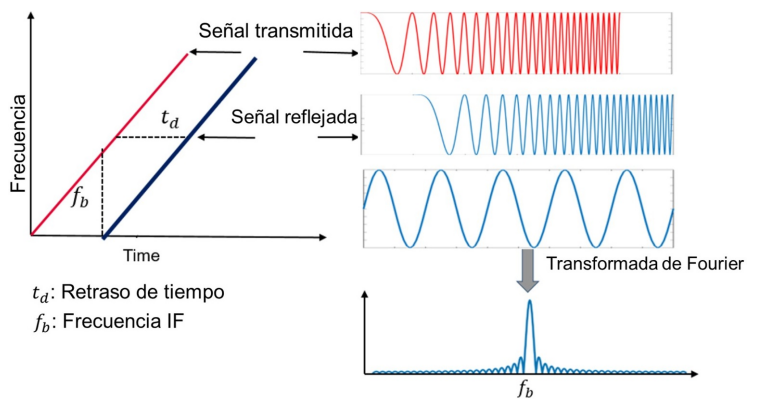

Figura 1. Principio básico de operación de un radar FMCW [15]

El valor de la distancia a un cuerpo se obtiene a partir de la Ecuación 1.

$$
R=\frac{c T_{c} f_{b}}{2 B}
$$

Donde:

$R$ es la distancia del objeto

$c$ es la rapidez de la luz

$T_{c}$ es el tiempo del chirp

$f_{b}$ es la frecuencia de la señal IF (beat)

$B$ es el ancho de banda 
La resolución de distancia o capacidad para resolver dos objetos cercanos se determina de acuerdo con la Ecuación 2.

$$
\triangle R=\frac{c}{2 B}
$$

Donde:

$\triangle R$ es la resolución

$c$ es la rapidez de la luz

$B$ es el ancho de banda

La distancia máxima puede determinarse en función de la frecuencia de muestro según la Ecuación 3.

$$
R_{\max }=\frac{f_{s} c}{2 S}
$$

Donde:

$R_{\max }$ es el alcance o distancia máxima

$f_{s}$ es la frecuencia de muestreo

$c$ es la rapidez de la luz

$S$ es la pendiente de la modulación $S=B / T_{c}$

Por otra parte, la velocidad máxima está dada por la Ecuación 4.

$$
v_{\max }=\frac{c}{4 f_{c} T_{c}}
$$

Donde:

$v_{\max }$ es la velocidad máxima

$c$ es la rapidez de la luz

$f_{c}$ es la frecuencia del chirp

$T_{c}$ es el tiempo del chirp

Ahora en caso de tener diferentes objetos se tendrán diferentes frecuencias, por lo tanto, en lugar de aplicar la Ecuación 1, una de las técnicas más comunes es realizar un análisis espectral a partir de la transformada rápida de Fourier (FFT) de acuerdo con la Figura 2 para obtener un mapa Range-Doppler.

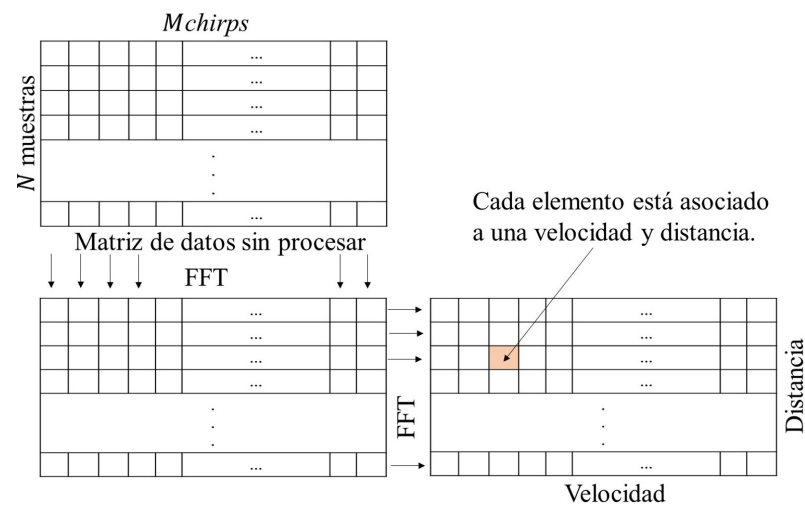

Figura 2. 2D Range-Doppler FFT
El ángulo de llegada (AoA por sus siglas en inglés) puede obtenerse a partir de una tercera transformada de Fourier (3D FFT), de forma que los picos en el espectro de frecuencia correspondan a un ángulo determinado, esto se realiza normalmente cuando se tienen cuatro antenas receptoras y una o más antenas transmisoras para obtener un arreglo de varias entradas y varias salidas (MIMO por sus siglas en inglés) [8]. Cuando el circuito cuenta con solo dos antenas receptoras es preferible obtener el AoA a partir de la Ecuación 5.

$$
\alpha=\sin ^{-1} \frac{\lambda \triangle \varphi}{2 \pi d}
$$

Donde:

$\alpha$ es el ángulo de llegada

$\lambda$ es la longitud de onda

$\triangle \varphi$ es la diferencia de fase entre la señal de las antenas

$d$ es la distancia entre las antenas

\subsection{Aplicaciones de radares en la industria automotriz}

En los últimos años los radares se han empleado en vehículos principalmente por razones de seguridad como los sistemas ADAS, anticipando necesidades y tomando la iniciativa cuando es necesario [16]. En la industria automotriz las aplicaciones de los radares se dividen principalmente en radares de corto alcance (SRR) que permiten manejar detección de puntos ciegos (BSD), asistencia al cambio de vía (LCA), alerta de tráfico cruzado en las partes frontal y trasera (CTA), alerta de impacto lateral y alerta de ciclistas en la vía lateral (Figura 3). Por otra parte, se encuentran los radares de medio y largo alcance (MRR y LRR) responsables del frenado automático de emergencia (AEB) frente a colisiones con peatones y con otros vehículos, así como el control de crucero adaptativo (ACC) [17].

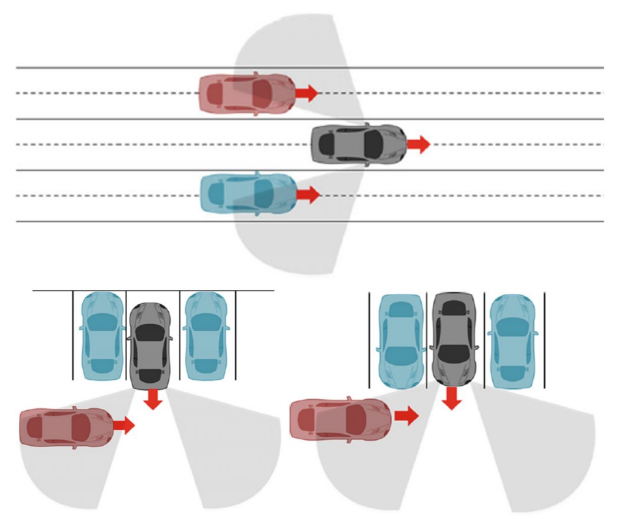

Figura 3. Ejemplo de escenario de corto alcance. Detección de puntos ciegos y alerta de tráfico cruzado 
El radar seleccionado en este trabajo, cuyas características se detallan más adelante, es de corto alcance por lo que responde a los requerimientos típicos que se muestran en la Tabla 1.

Tabla 1. Requerimientos de radar de corto alcance [17]

\begin{tabular}{ccc}
\hline $\begin{array}{c}\text { Requerimientos de los } \\
\text { parámetros del radar }\end{array}$ & $\begin{array}{c}\text { Requerimientos } \\
\text { BSD }\end{array}$ & $\begin{array}{c}\text { Requerimientos } \\
\text { CTA }\end{array}$ \\
\hline Alcance $(\mathrm{m})$ & $1-50$ & $2-60$ \\
Exactitud en alcance $(\mathrm{m})$ & $\pm 0,10$ & pm0,20 \\
Resolución en alcance $(\mathrm{m})$ & 0,75 & 1 \\
Velocidad $(\mathrm{m} / \mathrm{s})$ & -70 to +70 & -70 to +70 \\
Exactitud en velocidad $(\mathrm{m} / \mathrm{s})$ & $\pm 0,1$ & $\pm 0,1$ \\
Resolución en velocidad $(\mathrm{m} / \mathrm{s})$ & 0,25 & 0,3 \\
Azimut $\left({ }^{\circ}\right)$ & \pm 75 & \pm 40 \\
Exactitud en azimut $\left({ }^{\circ}\right)$ & \pm 5 & \pm 5 \\
Resolución en azimut $\left({ }^{\circ}\right)$ & 15 & 15 \\
Elevación $\left(^{\circ}\right)$ & \pm 6 & \pm 10 \\
Exactitud elevación $\left(^{\circ}\right)$ & pm0,1 & \pm 5 \\
Resolución en elevación $\left(^{\circ}\right)$ & - & - \\
\hline
\end{tabular}

\subsection{Herramientas didácticas y el aprendizaje orientado a competencias}

Dentro de los aspectos que involucran la enseñanza a nivel de ingeniería cobra vital importancia las prácticas de laboratorio. Las cuales según [18] deben ser capaces de proporcionar información suficiente que permita definir y caracterizar actividades en las cuales los estudiantes desarrollen competencias específicas y transversales.

Por ejemplo, Arduino en los últimos años ha sido empleado con frecuencia gracias a su facilidad de manejo y bajo de costo de adquisición [19]. Esto es algo que influye en países donde no se cuentan con los recursos adecuados para adquirir equipos que pueden ser bastantes costosos, por ello emplear tarjetas de desarrollo de bajo costo, en este caso de radar, puede generar amplios beneficios. En específico permiten mejorar el acceso a dispositivos que, como se ha mencionado, han sido de uso casi exclusivo en el área militar y aeronáutica. Asimismo, permite mejorar el acceso a tecnología pionera que se está desarrollando en los vehículos de alta, media e incluso de baja gama. En este sentido, es importante que en América Latina se prepare de forma adecuada a los futuros ingenieros y técnicos en los conocimientos y competencias para poder realizar mantenimiento a los vehículos y, de igual forma, participar en investigación y desarrollo en estas áreas en vista de que en la actualidad los automóviles están equipados con una gran de dispositivos electrónicos [20].

Por otra parte, la enseñanza de la teoría se beneficia y armoniza con metodologías experimentales adecuadas, no solo en los laboratorios tradicionales (p. ej. electrónica o circuitos eléctricos), sino aquellas asignaturas que, tradicionalmente, no involucran actividades experimentales como las Matemáticas.

\section{Materiales y métodos}

En la actualidad diversas empresas tecnológicas han sacado al mercado tarjetas que en un principio sirven para evaluar el desempeño de los circuitos integrados que ofrecen, tal es el ejemplo de Analog Devices que ofrece la Demorad para diversos chipset incluidos el ADF5901 (circuito integrado de microondas a $24 \mathrm{GHz}$ con 2 canales para transmisión), ADF5904 (receptor de 4 canales a $24 \mathrm{GHz}$ ) y AD4159 (encargado de generar las rampas triangulares o diente de sierra) y otros circuitos que permiten un completo sistema de radar integrado en una tarjeta [21]. También Infineon ofrece las tarjetas Distance2Go [22] o Position2Go [23], entre otras.

Para este trabajo se ha optado por la tarjeta Position2Go considerando que es una de las más sencillas (desde el punto de arquitectura) que se pueda encontrar en el mercado, con un uso relativamente fácil y un costo asequible. Se evalúa su desempeño para obtener mapas RangeDoppler, posición y detección de objetos mediante el algoritmo CFAR (Constant False Alarm Rate) o tasa de falsa alarma constante que es uno de los más sencillos de implementar y comprender desde el punto de vista didáctico.

\section{1. hardware}

La tarjeta Position2Go incluye todos los elementos necesarios para la generación, recepción y procesamiento de las señales. Esto dividido en cuatro secciones importantes: la parte de radiofrecuencia (RF) incluye el CI BGT24MTR12 que es el principal encargado de generar y recibir las señales a $24 \mathrm{GHz}$ [23] así como tres antenas, una para la transmisión y dos para la recepción. También cuenta con amplificadores analógicos que permiten la interfaz entre la RF y la parte digital. Incluye una parte de control de frecuencia y una parte digital, específicamente cuenta con el microcontrolador XMC4700 ARM de 32 bits para muestrear y procesar los datos, todo esto montado en una placa que permite el acceso mediante una conexión USB 2.0 que también posee conexión CAN en caso de que se requiera una comunicación directa con una unidad de control electrónica (ECU) de un vehículo. Por lo tanto, se puede decir que es todo un sistema de radar integrado que permite su control y programación directamente mediante algunos de los protocolos de conexión señalados (en este trabajo se ha empleado el USB). En la Figura 4 se muestra la arquitectura general de la tarjeta, mientras que en la Figura 5 se muestra la tarjeta empleada en este trabajo. 


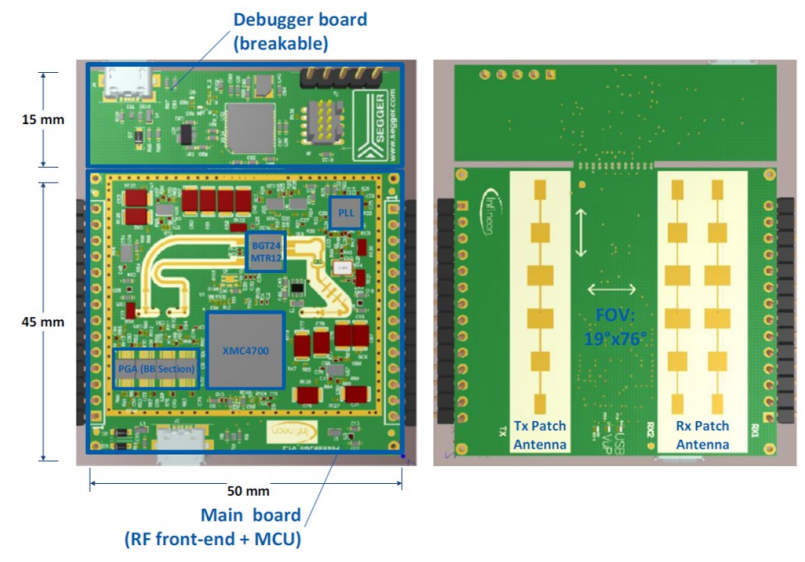

Figura 4. Tarjeta Position2Go

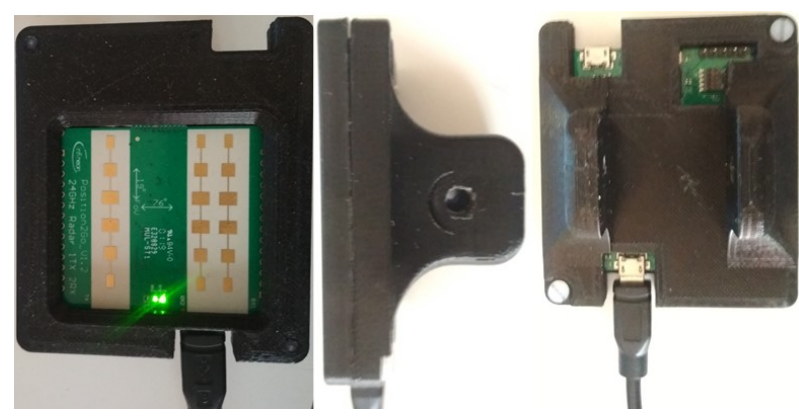

Figura 5. Tarjeta Position2Go empleada

\subsection{Diseño experimental}

Position2Go incluye un firmware que facilita el control de los diferentes CI y periféricos a través del microcontrolador XMC4700. Este firmware permite realizar cambios en la configuración de parámetros de interés y proporcionar las señales procesadas o no. Existe una interfaz de usuario para obtener directamente la señal procesada, generando el espectro de frecuencia y determinando la posición y velocidad de diferentes objetos [24]. Este toolbox de Infineon (Figura 6) permite mostrar los cálculos realizados por el microcontrolador en cuenta que por defecto los cálculos los realiza con un límite o threshold de 100 LSB que resulta en un alcance de aproximadamente $12 \mathrm{~m}$ para peatones y $15 \mathrm{~m}$ para una sección equivalente de radar (RCS) de $1 \mathrm{~m}^{2}$ [25]. No obstante, trabajar con esta interfaz limita la capacidad de procesar las señales, además, no permite obtener las ventajas didácticas que se buscan (aunque puede ser un punto de partida). Por lo anterior, se plantea adquirir la señal IF y procesarla directamente en MATLAB. Para esto se dispone de una API que permite modificar y obtener valores directamente [26]. En la Tabla 2 se muestran las características seleccionadas gracias a diversas líneas de códigos implementadas.

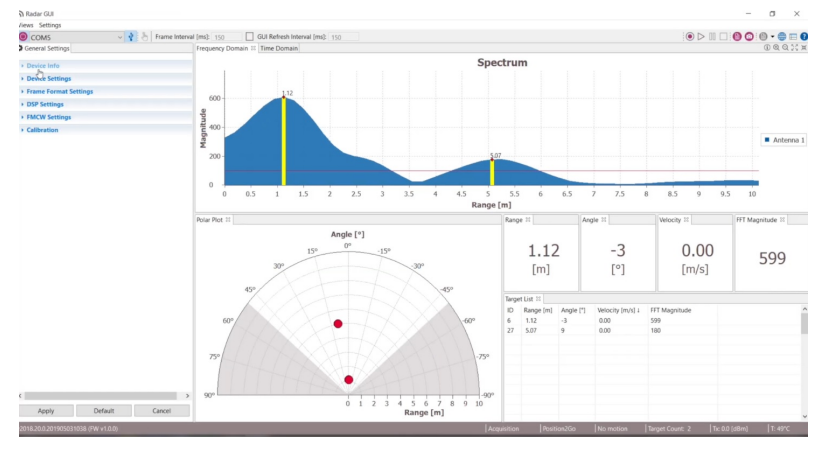

Figura 6. Radar GUI de Infineon

Tabla 2. Parámetros del sistema

\begin{tabular}{|c|c|}
\hline Parámetros & Valor \\
\hline $\begin{array}{l}\text { Tiempo de subida de rampa } \\
\text { (up-chirp): } \tau u p\end{array}$ & $301 \mu s$ \\
\hline $\begin{array}{l}\text { Tiempo de bajada de rampa } \\
(\text { down-chirp }): \tau_{\text {down }}\end{array}$ & $100 \mu s$ \\
\hline $\begin{array}{l}\text { Tiempo de espera entre rampas } \\
\tau_{s b y}\end{array}$ & $100_{\mu s}$ \\
\hline $\begin{array}{l}\text { Tiempo de repetición de pulso: } \\
\qquad \tau_{u p}+\tau_{\text {down }}+\tau_{s b y}\end{array}$ & $501_{\mu s}$ \\
\hline Ancho de banda $(B)$ & $301 M H z$ \\
\hline Frecuencia de muestreo $\left(f_{s}\right)$ & $850 \mathrm{MHz}$ \\
\hline $\begin{array}{l}\text { Muestreos por rampa } \\
\text { (simples per chirp) }\end{array}$ & 256 \\
\hline $\begin{array}{l}\text { Rampa por cuadro } \\
\text { (chirps per frame) }\end{array}$ & 16 (máximo) \\
\hline Resolución de alcance $(\triangle R)$ & $75 \mathrm{~cm}^{*}$ \\
\hline Alcance mínimo $\left(R_{\min }\right)$ & $0 \mathrm{~m}$ \\
\hline Alcance máximo $\left(R_{\max }\right)$ & $14 m^{* *}$ \\
\hline
\end{tabular}

Cabe destacar que, las condiciones detalladas no son típicas para la evaluación de radares. Sin embargo, se ha preferido un escenario más cotidiano y adaptable a la realidad universitaria por tres motivos.

El primero se debe a que en un ambiente de laboratorio universitario es difícil tener acceso a condiciones reglamentadas para una evaluación correcta, como la disposición de una cámara anecoica (Figura 7) y reflectores cuadrados o triangulares para evitar dispersiones en las medidas y así evaluar de forma apropiada la exactitud y precisión del radar; así como vehículos para pruebas, un vehículo estático y un peatón son condiciones más factibles de tener en un ambiente educativo. El segundo motivo es que los radares FMCW a $24 \mathrm{GHz}$ son empleados para corto alcance y bajas velocidades según se ha detallado anteriormente. Finalmente, el principal objetivo didáctico planteado es la comprensión y puesta en práctica de los aspectos teóricos y no de una evaluación profunda de las características propias de la tarjeta empleada.

Como aspecto adicional es menester mencionar que, debido a la limitación de libre circulación y el confi- 
namiento durante gran parte del 2020 (especialmente entre los meses de marzo y junio) y el cierre de las universidades, las mediciones fueron realizadas en un ambiente no ideal, por la imposibilidad de acceder al material de trabajo que se encontraba en los laboratorios.

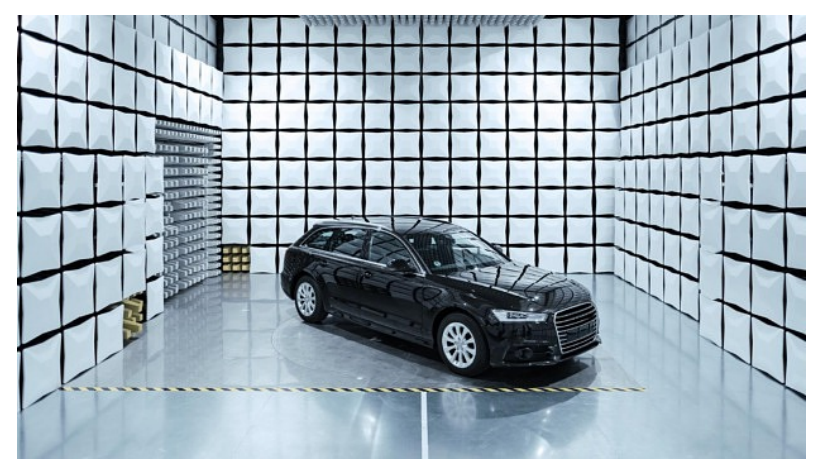

Figura 7. Cámara anecoica $[27]$

Para realizar las mediciones se ha considerado el siguiente escenario de acuerdo con la Figura 8, donde se ha tomado en cuenta un vehículo estático a una distancia de $2,9 \mathrm{~m}$, un peatón estático a una distancia de $7,5 \mathrm{~m}$, una pared a una distancia de $12,3 \mathrm{~m}$, además de un peatón que recorre corriendo en línea recta desde la posición inicial del radar hasta la pared y regresa.

A partir de la configuración detallada en la Figura 8 se realizaron diversas mediciones considerando los datos (señal IF) que suministra el firmware directamente a MATLAB, donde cada medición tiene 50 dataframes mediante un bucle for que puede ser modificado para obtener la cantidad deseada. Un dataframe consiste en los datos puros (señal IF) a ser procesados en diversas funciones implementadas en MATLAB.

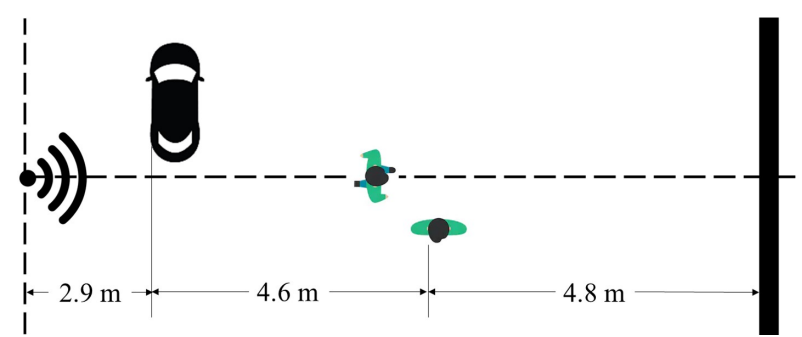

Figura 8. Escenario de medición

\subsection{Procesamiento en MATLAB}

El procesamiento de datos puede resumirse en 4 niveles. Primero se deben definir los parámetros detallados en la Tabla 2 siguiendo los métodos listados en [26], calcular los límites de medición a partir de las ecuaciones detalladas, definir la ventana a aplicar que en este caso se ha optado por una ventana Hanning con el propósito de reducir la amplitud de los lóbulos adyacentes al pico principal en la FFT, reorganizar los datos, esto debido a que el firmware envía los datos por defecto en un arreglo de tres dimensiones. Donde la primera dimensión contiene todas las muestras por rampa (chirp) para la estimación de distancia; la segunda corresponde a las diferentes rampas por cuadro (chirps per frame) para la estimación de velocidad y la tercera, a la antena; sin embargo, se ha optado por trabajar en dos arreglos de tres dimensiones, uno para cada antena y sustituyendo la tercera dimensión por el número de medición de forma tal que se obtuvieron dos arreglos de tamaño $N \times M \times L$, siendo $N$ la cantidad de muestras por chirps limitadas en frecuencia correspondiente al intervalo de distancia ([2,14] metros); M la cantidad de muestras por frame, esto corresponde a $M=16 \times 256=4096$; y finalmente $L$ mediciones siendo en este caso $L=50$ mediciones. El intervalo entre mediciones ha sido de $0.2 \mathrm{~s}$.

Luego se ha aplicado una primera FFT al primer chirp de forma que se obtenga el espectro de distancia con un tamaño de $2^{12}$, por lo tanto, se rellenan con ceros $2^{12}-N$ elementos mediante zero padding, lo cual se realiza automáticamente en MATLAB (ver [28]), el propósito es aumentar la resolución de manera tal que sea más sencillo reconocer dos frecuencias cercanas [29]. Esto resulta en la llamada Range-FFT.

Posteriormente se aplica una nueva FFT, pero esta vez a través de los diferentes chirps de forma tal que se obtenga el mapa Range-Doppler.

Finalmente, se ha aplicado el algoritmo CFAR para identificar los diferentes objetos a partir de lo detallado en [30]. Esta tarjeta presenta la desventaja de ser limitada en ancho de banda (200 MHz para $24 \mathrm{GHz})$ y en cantidad de chirps/frame, por lo tanto, el tamaño de las celdas para la aplicación del CFAR debe ser elevado, en este caso se optó por $90 \times 90$ de guardia y $30 \times 30$ de entrenamiento, con un factor $K=10^{8 / 20}$. Un diagrama de flujo con una generalización del algoritmo empleado es mostrado en la Figura 9.

La programación se ha desarrollado a partir de demos de Infineon [23], Analog Devices [21] y el trabajo de Guerrero [11] aplicado a otra tarjeta de desarrollo. Sin embargo, estos programas han sido modificados en grandes rasgos para incluir:

Capacidad de exportar grabaciones (.AVI) en MATLAB

Capacidad de crear y exportar gráficos polares de la posición de los cuerpos.

Capacidad de aislar y analizar dataframes deseados.

Aplicación de algoritmo CFAR. 
Adquisición de datos sin procesar

Reorganización de datos

\begin{tabular}{|c|}
\hline Aplicación de Ventana Hanning \\
\hline Zero Padding \\
\hline Range FFT \\
Procesamiento de distancia (Range map) \\
\hline
\end{tabular}

\begin{tabular}{|c|}
\hline Aplicación de Ventana Hanning \\
\hline Zero Padding \\
\hline $\begin{array}{c}\text { Doppler FFT } \\
\text { Procesamiento de velocidad } \\
\text { (mapa Range-Doppler) }\end{array}$ \\
\hline
\end{tabular}

\begin{tabular}{|c|}
\hline CFAR \\
\hline Cálculo de centroides \\
\hline \begin{tabular}{c} 
Scatter de puntos en plano \\
velocidad-distancia \\
Identificación de objetos \\
\hline
\end{tabular} \\
\hline
\end{tabular}

Figura 9. Diagrama de procesamiento de señal

\section{Resultados y discusión}

\subsection{Firmware e Infineon Toolbox}

A partir de los datos directamente procesados por el microcontrolador se obtuvo el mapa de puntos mostrado en la Figura 10, en el cual se evidencia varias detecciones falsas (false detection) o detecciones erróneas (misdetection) debido a que el threshold es fijo por defecto según se ha detallado anteriormente.

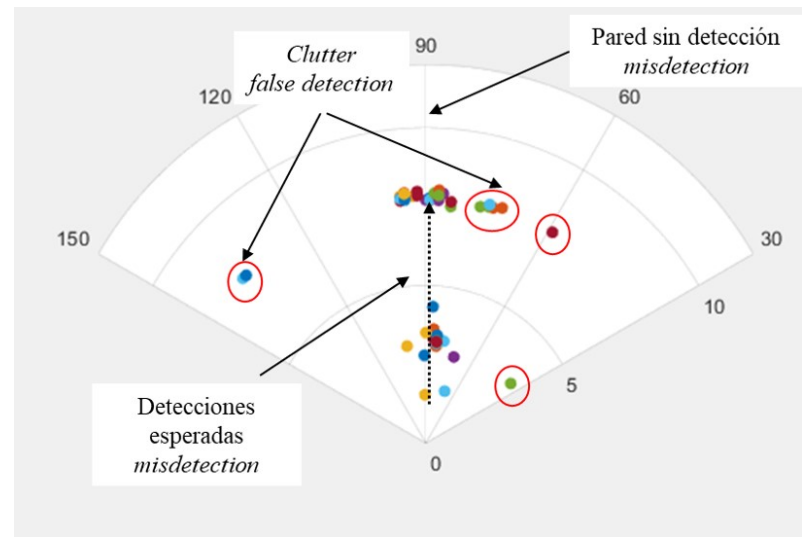

Figura 10. Mediciones a partir del firmware
Por otra parte, aunque esta primera aproximación para los estudiantes puede parecer suficiente, el hecho de que solo se muestren los datos ya procesados limita los objetivos que se buscan al utilizar la tarjeta, es decir, no se evalúa didácticamente la obtención de los resultados, sino que solo son mostrados sin profundizar en el algoritmo empleado para ello. Aunque una modificación del algoritmo y una implementación directa del CFAR en el microcontrolador es posible, su implementación no es sencilla y por este motivo el resto de los resultados mostrados fueron obtenidos a partir de la implementación en MATLAB.

\subsection{MATLAB}

El mapa Range-Doppler de la Figura 11 se ha seleccionado entre los 50 generados, en él es posible apreciar el inconveniente de ancho de banda de los radares de $24 \mathrm{GHz}$ de $200 \mathrm{MHz}$ y es que no poseen una buena resolución en distancia, sumado a que por defecto los chirps por frame de la Position2Go están limitados a 16, cuando en otras tarjetas de $77 \mathrm{GHz}$, por ejemplo, se pueden obtener hasta 128 por frame.

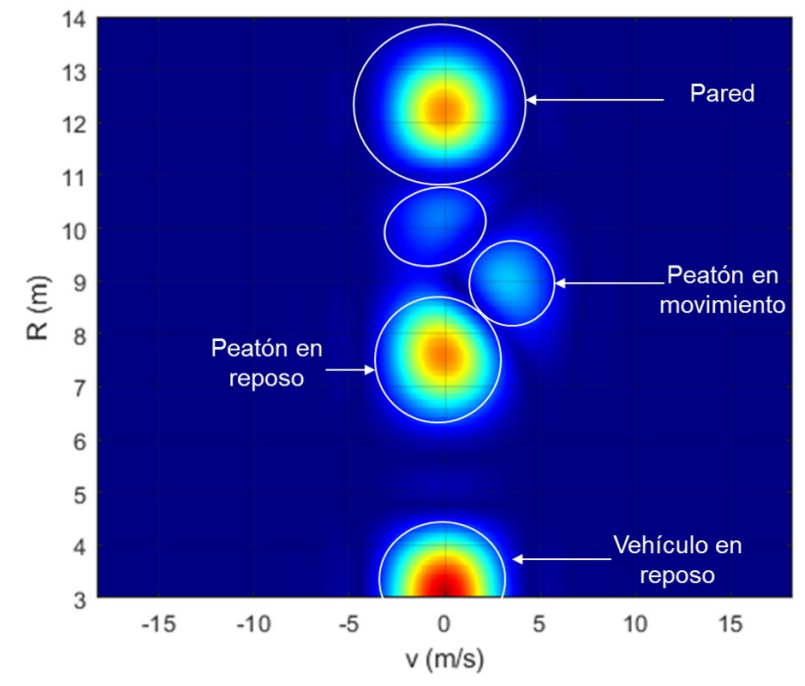

Figura 11. Mapa Range-Doppler

En la Figura 11 se pueden observar cinco áreas en correspondientes a máximos relativos en la FFT, la identificación mediante algoritmos que permitan discriminar automáticamente entre los diferentes cuerpos que puedan estar en el entorno se encuentran fuera del alcance de este trabajo, particularmente debido a que el estudio se ha planteado como herramientas en ambientes de pregrado, lo que implica una complejidad limitada.

En este punto sería importante aclarar al estudiante que si bien este mapa permite una identificación rápida de forma visual no es muy práctico desde el punto de vista computacional puesto que un microcontrolador por sí solo no sería capaz de identificar los cuerpos sin 
un algoritmo adicional que se encargue de ello (en [31] se detalla con profundidad uno de estos algoritmos). Es en este punto donde la aplicación del algoritmo CFAR facilita obtener un límite de detección de forma dinámica de forma tal que se tome en cuenta la potencia con respecto al ruido (SNR). Cabe destacar que el CFAR por sí solo no permite hacer la discriminación acerca de los cuerpos (vehículos, peatones, motos, etc.).

Dado que el factor $\mathrm{K}$ ha sido configurado relativamente bajo de allí que resulte una gran cantidad de áreas al aplicar el CFAR (Figura 12). Para solucionar esto se han tomado solo aquellos puntos que tengan una potencia de al menos $-40 \mathrm{~dB}$, valor obtenido empíricamente a partir de diversas mediciones (para una menor potencia generalmente se trató de detecciones falsas), este valor también puede ser dinámico, considerando que la potencia reflejada depende de la ecuación de radar que a su vez depende de la distancia a la que se encuentre el cuerpo, al mismo tiempo, los niveles de reflexión son diferentes de acuerdo con el objeto, por ejemplo, un vehículo refleja más potencia que un peatón, partiendo de que las ondas electromagnéticas (como las transmitidas por un radar) se reflejan en los cuerpos de acuerdo con su sección equivalente de radar que depende de diversos factores tales como el área, el material (de la ropa o el vehículo), la forma, etc., esto puede servir para poder clasificar los cuerpos. En la Tabla 3 se muestran algunos de los valores obtenidos a partir de los centroides para cada una de las áreas (Figura 13).

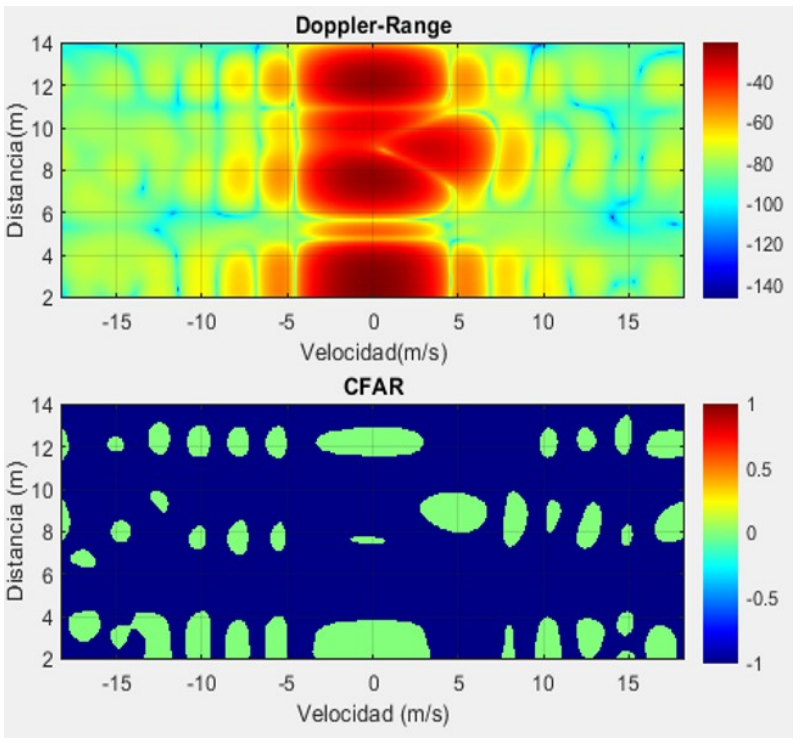

Figura 12. Mapa Range-Doppler en dB y resultado de CFAR

En la Figura 14 se muestran los objetos detectados considerando el límite de $-40 \mathrm{~dB}$. Es evidente entonces que el CFAR permite identificar objetos de forma correcta aun cuando la velocidad del peatón ha sido un poco más alta de la real (Tabla 4); en este sentido, esto es algo que este trabajo aporta a la detección de objetos en relación con la realizada en la interfaz gráfica de Infineon. Es de aclarar que los resultados no han sido optimizados, de esta manera, se pueden mostrar condiciones en las que un estudiante podría implementar un algoritmo no optimizado e incluso así obtener unos resultados aceptables.

Tabla 3. Datos ordenados considerando la potencia

\begin{tabular}{ccc}
\hline $\begin{array}{c}\text { Distancia } \\
(\mathbf{m})\end{array}$ & $\begin{array}{c}\text { Velocidad } \\
(\mathbf{m} / \mathbf{s})\end{array}$ & $\begin{array}{c}\text { Potencia } \\
(\mathbf{d B})\end{array}$ \\
\hline 2,8564 & $-0,0803$ & $-21,1564$ \\
7,5806 & $-0,2945$ & $-23,2399$ \\
12,2498 & $-0,0803$ & $-23,3293$ \\
8,9539 & 4,8109 & $-33,7255$ \\
2,9114 & $-5,6320$ & $-53,0894$ \\
$\vdots$ & $\vdots$ & $\vdots$ \\
\hline
\end{tabular}

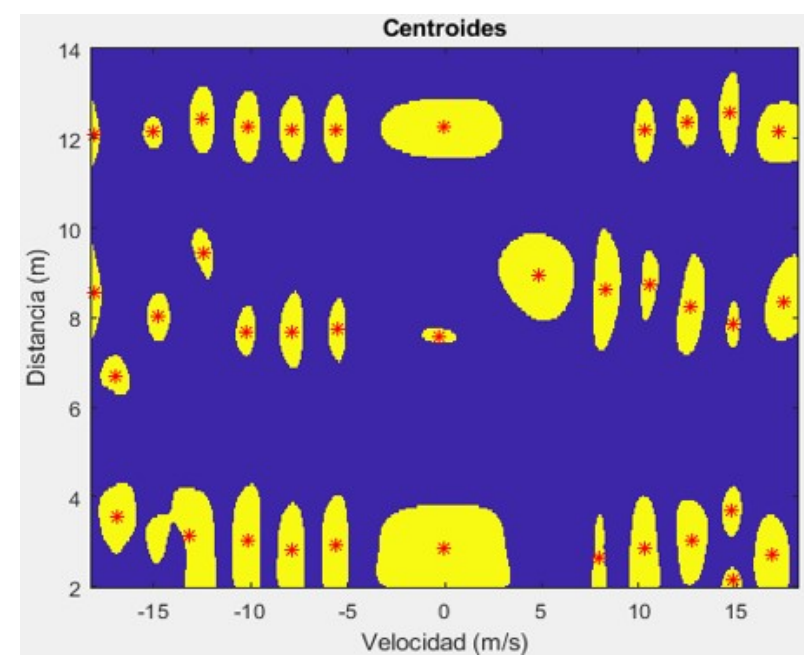

Figura 13. Datos ordenados considerando la potencia

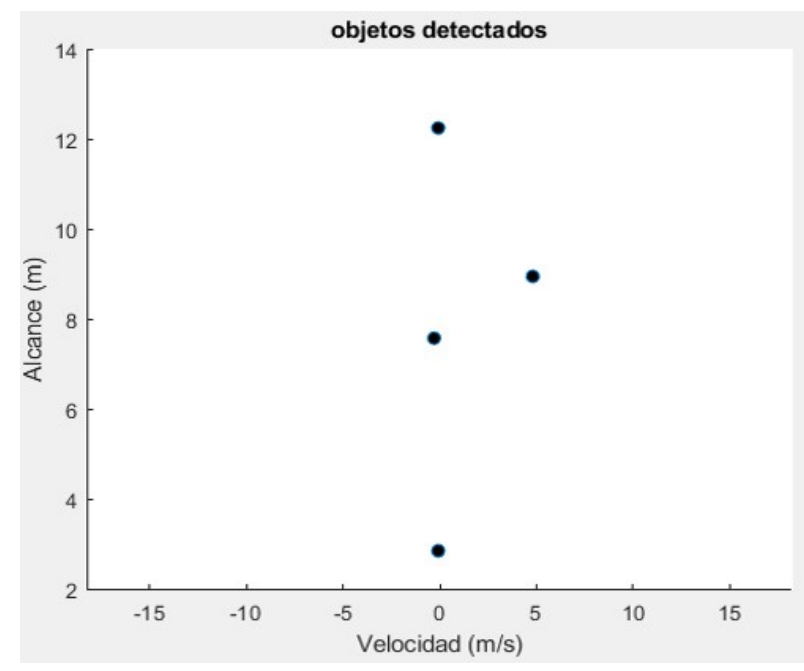

Figura 14. Identificación de objetos mediante CFAR 
Tabla 4. Datos ordenados considerando la potencia

\begin{tabular}{ccccc}
\hline & $\begin{array}{c}\text { Distancia } \\
\text { medida }(\mathbf{m})\end{array}$ & $\begin{array}{c}\text { Distancia } \\
\text { real }(\mathbf{m})\end{array}$ & $\begin{array}{c}\text { Velocidad } \\
\text { medida }(\mathbf{m} / \mathbf{s})\end{array}$ & $\begin{array}{c}\text { Velocidad } \\
\text { real }(\mathbf{m} / \mathbf{s})\end{array}$ \\
\hline $\begin{array}{c}\text { Vehículo } \\
\text { Peatón en }\end{array}$ & 2,8564 & 2,9 & $-0,0803$ & 0 \\
reposo & 7,5806 & 7,5 & $-0,2945$ & 0 \\
$\begin{array}{c}\text { Pared } \\
\text { Peatón en }\end{array}$ & 12,2498 & 12,3 & $-0,0803$ & 0 \\
movimiento & 8,9539 & $\approx 9$ & 4,8109 & $\approx 4$ \\
\hline
\end{tabular}

Las Figuras 11-14 muestran uno de los cuadros (frames) de una de las mediciones que ha sido seleccionada, por motivos de la estructura del presente documento no pueden ser mostrados en su totalidad. Sin embargo, en las Figuras 15 y 16 se muestran las distancias obtenidas a partir de la aplicación del CFAR en cada uno de los dataframe. La Figura 15 muestra el peatón en movimiento, donde se tiene una tasa efectiva de detección de 78,57 \% (sin considerar las detecciones falsas y las no detecciones). Por otra parte, en la Figura 17 se muestra la dispersión para los cuerpos en reposo. En la Tabla 5 se detallan algunos valores estadísticos que se encuentran dentro de los valores de la Tabla 1. No obstante, es importante mencionar que la precisión y exactitud mostrada no necesariamente refleja los valores reales del radar, debido a las restricciones mencionadas con anterioridad. La importancia de estos resultados radica en que aún en condiciones no óptimas, el radar logra tener una buena precisión y exactitud, por lo tanto, se espera que los estudiantes logren resultados adecuados sin recurrir a una calibración profunda y algoritmos más complejos.

A partir de estos resultados, el empleo de esta tarjeta de evaluación se perfila como una herramienta adecuada a usar en prácticas de laboratorio puesto que abarca competencias específicas: la comprensión de fundamentos matemáticos como la transformada de Fourier, números complejos, operaciones matriciales, análisis espectral, entre otros, así como fundamentos físicos: cinemática, ondas electromagnéticas, efecto Doppler, etc. Por otra parte, con una adecuada guía del docente se puede fomentar competencias transversales de acuerdo con [32], tales como: conocimientos de informática relativos al ámbito de estudio, específicamente se plantea el uso de MATLAB y otras herramientas informáticas. Asimismo, se puede fomentar el trabajo en equipo, aprendizaje autónomo, adaptación a nuevas situaciones, capacidad de aplicar conocimientos teóricos en la práctica, uso de Internet como fuente de información, entre otras.

Todo lo anterior en un ambiente adecuado, que no genere frustración en los alumnos y una tutoría conveniente puede traducirse en un gran impacto en la formación de estos.

Desafortunadamente no se han encontrado casos de estudios anteriores específicos acerca de la viabilidad de la propuesta del uso de radares como herramienta didáctica y la evaluación del impacto. Es por ello por lo que en este trabajo se analiza la viabilidad tanto técnica como didáctica de emplear una tarjeta de evaluación de las numerosas que se encuentran en el mercado.

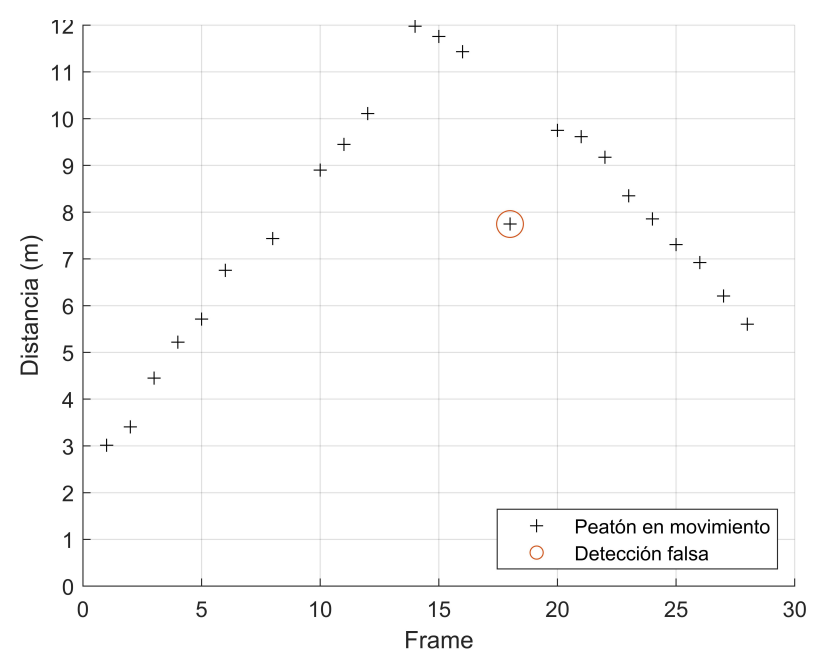

Figura 15. Identificación de objetos mediante CFAR

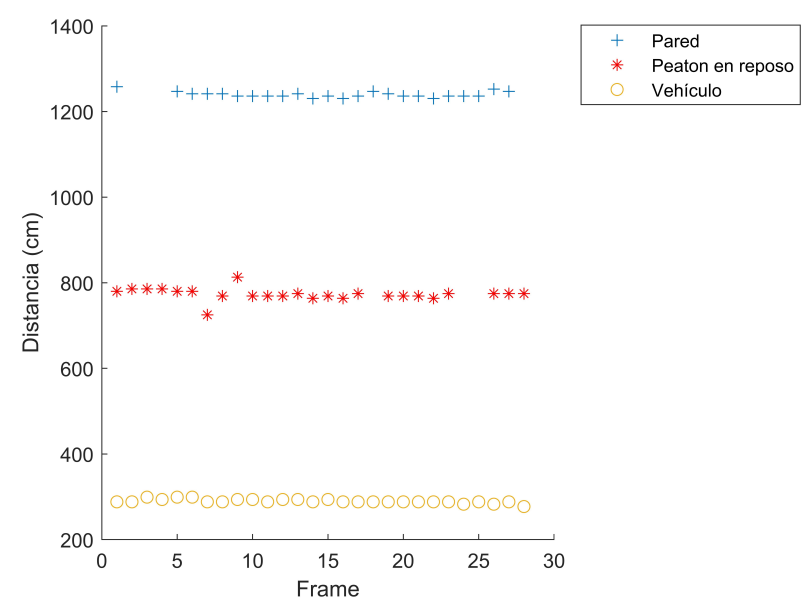

Figura 16. Identificación de objetos mediante CFAR
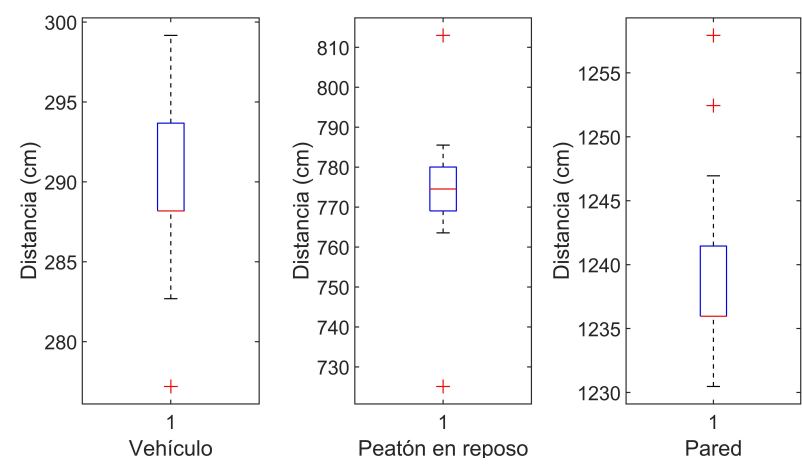

Figura 17. Identificación de objetos mediante CFAR 
Tabla 5. Estadísticos para la medición de distancia de los cuerpos en reposo

\begin{tabular}{cccc}
\hline \multicolumn{4}{c}{ Distancia } \\
\hline Real (cm) & Media (cm) & Mediana (cm) & $\begin{array}{c}\text { Desviación } \\
\text { estándar }(\mathbf{c m})\end{array}$ \\
\hline 290 & 289,75 & 288,18 & 4,84 \\
750 & 773 & 774,54 & 14,03 \\
1230 & 1239,40 & 1235,96 & 6,69 \\
\hline
\end{tabular}

\section{Conclusiones}

Este trabajo muestra una tarjeta de evaluación basada en un radar FMCW que opera a $24 \mathrm{GHz}$ con un ancho de banda de $200 \mathrm{MHz}$. El desempeño empírico sin considerar un análisis estadístico que permita determinar la tasa de detecciones incorrectas y sin una calibración precisa es más que aceptable para su uso como herramienta educativa con el propósito de entender el funcionamiento de los radares de corto alcance y su aplicación en vehículos. La obtención de los datos y el procesamiento de estos son relativamente sencillos, pudiendo desarrollarse tanto como el investigador o docente así lo desee. Sin embargo, se recomienda iniciar por la aplicación de un algoritmo que involucre la transformada rápida de Fourier en dos dimensiones para obtener mapas RangeDoppler y luego mediante un algoritmo CFAR mejorar la detección de objetos con respecto a la implementación de un límite fijo. En caso de querer profundizar en la clasificación de cuerpos, se recomienda investigar acerca de los diferentes algoritmos disponibles.

Aunque no se ha comparado con otras tarjetas, los resultados sumados al bajo costo de la Position2Go muestran que la implementación de su uso en prácticas de laboratorio en las carreras de Ingeniería Automotriz y de Telecomunicaciones puede traer numerosos beneficios en el desarrollo de las capacidades específicas y transversales de los estudiantes.

\section{Referencias}

[1] V. Jain and P. Heydari, Automotive radar sensors in silicon technologies. Springer Science \& Business Media, 2012. [Online]. Available: https://bit.ly/3kak6ER

[2] J. Gamba, Radar Signal Processing for Autonomous Driving. Springer, 2020. [Online]. Available: https://bit.ly/3k8LP95

[3] C. Kumar and A. Killedar, mmWave Radar ADAS Applications, 2015. [Online]. Available: https://bit.ly/3p6XeK9

[4] M. Nazir, "Automotive radar target detection using ambiguity function," Ph.D. dissertation,
University of Birmingham, 2016. [Online]. Available: https://bit.ly/3n2kI1g

[5] P. Pursula and V. Viikari, "Novel automotive radar applications," in Millimetre Wave Days Proceedings, 2011, millimetre Wave Days 2011: 6th ESA Workshop on Millimetre-Wave Technology and Applications and 4th Global Symposium on Millimeter Waves; Conference date: 23-05-2011 Through 25-05-2011. [Online]. Available: https://bit.ly/2U5GZi0

[6] N. Bouhlel, S. Meric, C. Moullec, and C. Brousseau, "FMCW radar system for transponder identification," Progress In Electromagnetics Research B, vol. 81, pp. 101-122, 2018. [Online]. Available: http: //dx.doi.org/10.2528/PIERB18032007

[7] F. Ali and M. Vossiek, "Detection of weak moving targets based on 2-D range-doppler FMCW radar Fourier processing," in German Microwave Conference Digest of Papers, 2010, pp. 214-217. [Online]. Available: https://bit.ly/3ni0lgE

[8] J. Kim, J. Chun, and S. Song, "Joint range and angle estimation for FMCW MIMO radar and its application," 2018. [Online]. Available: https://bit.ly/2Uk63Ck

[9] B. Kim, Y. Jin, S. Kim, and J. Lee, "A lowcomplexity FMCW surveillance radar algorithm using two random beat signals," Sensors (Basel), vol. 19 , no. 3 , p. 608,2019 . [Online]. Available: https://dx.doi.org/10.3390\%2Fs19030608

[10] J. Svensson, "High resolution frequency estimation in an FMCW radar application," 2018. [Online]. Available: https://bit.ly/2UjCXTs

[11] E. Guerrero-Menéndez, "Frequency-modulated continuous-wave radar in automotive applications," 2018. [Online]. Available: https: //bit.ly/3pp6slg

[12] M. D. Fontaine, Traffic Monitoring, 2009. [Online]. Available: https://bit.ly/36ne6nm

[13] V. Issakov, Microwave Circuits for $24 \mathrm{GHz}$ Automotive Radar in Silicon-based Technologies. Springer, 2010. [Online]. Available: https://bit.ly/35nfJST

[14] C. Händel, H. Konttaniemi, and M. Autioniemi, State-of-the-Art Review on Automotive Radars and Passive Radar Reflectors Arctic Challenge research project. Lapland University of Applied Sciences, 2018. [Online]. Available: https://bit.ly/36u2yig 
[15] Infineon, "Position2go software user manual," Infineon, Tech. Rep., 2019. [Online]. Available: https://bit.ly/38ASmqE

[16] Mikusova, Miroslava, "Crash avoidance systems and collision safety devices for vehicle occupants," MATEC Web Conf., vol. 107, p. 00024, 2017. [Online]. Available: https: //doi.org/10.1051/matecconf/201710700024

[17] K. Ramasubramanian and K. Ramaiah, "Moving from legacy $24 \mathrm{ghz}$ to state-of-the-art 77-ghz radar," ATZelektronik worldwide, vol. 13, no. 3, pp. 46-49, Jun. 2018. [Online]. Available: https://doi.org/10.1007/s38314-018-0029-6

[18] J. M. Canino, V. Mena, J. Alonso, A. Ravelo, and E. García, "Prácticas de laboratorio en contextos de enseñanza-aprendizaje basados en competencias: dificultades y oportunidades," in I Jornadas Iberoamericanas de Innovación Educativa en el ámbito de las TIC Las Palmas de Gran Canaria, 2014. [Online]. Available: https://accedacris.ulpgc.es/bitstream/ 10553/15852/1/0719136_00000_0028.pdf

[19] S. Kocijancic, "Contemporary challenges in teaching electronics to stem teachers," AIP Conference Proceedings, vol. 2043, no. 1, p. 020002, 2018. [Online]. Available: https://doi.org/10.1063/1.5080021

[20] M. V. Noroña M. and M. F. Gómez B., "Desarrollo e innovación de los sistemas mecatrónicos en un automóvil: una revisión," Enfoque UTE, vol. 10, pp. 117-127, 03 2019. [Online]. Available: https://doi.org/10.29019/enfoqueute.v10n1.350

[21] Analog Devices, "Radar demonstration platform. evaluates radar chipset including the ADF5901, ADF5904 and ADF4159." Analog Devices, Tech. Rep., 2019. [Online]. Available: https://bit.ly/2YRxHK8.
[22] Infineon, "Demo distance2go," Infineon, Tech. Rep., 2020. [Online]. Available: https://bit.ly/ $35 \mathrm{Rr} 7 \mathrm{VI}$

[23] — , "Demo position2go," Infineon, Tech. Rep., 2020. [Online]. Available: https://bit.ly/2SUt6D8

[24] — "Infineon toolbox," Infineon, Tech. Rep., 2020. [Online]. Available: https://bit.ly/3lrUbtJ

[25] — , "Application notes," Infineon, Tech. Rep., 2020. [Online]. Available: https://bit.ly/3bmpDnt

[26] — "MATLAB radar system API," Infineon, Tech. Rep., 2020. [Online]. Available: https://bit.ly/38z2aS5

[27] CETECOM, "Testing services for various radar applications," CETECOM, Tech. Rep., 2020. [Online]. Available: https://bit.ly/2UwRWK1

[28] MATLAB. (2020) Fourier transforms. [Online]. Available: https://bit.ly/2YPQzcz

[29] S. Hilbert. (2013) FFT zero padding. [Online]. Available: https://bit.ly/36HV2R5

[30] MATLAB. (2020) Constant false alarm rate (CFAR) detection. [Online]. Available: https://bit.ly/3dCuWRf.

[31] C. Will, P. Vaishnav, A. Chakraborty, and A. Santra, "Human target detection, tracking, and classification using 24-GHz FMCW radar," IEEE Sensors Journal, vol. 19, no. 17, pp. 7283-7299, 2019. [Online]. Available: https://doi.org/10.1109/JSEN.2019.2914365

[32] M. Sepúlveda, "Las competencias transversales, base del aprendizaje para toda la vida," Universidad EAN, 2017. [Online]. Available: https://bit.ly/2GYyDGg 


\title{
SiMULACIÓN NUMÉRICA DEL FLUJO SUBEXPANDIDO EN LA TOBERA CÓNICA EXPERIMENTAL HELIOS-X
}

\author{
NUMERICAL SIMULATION OF THE \\ UNDER-EXPANDED FLOW IN THE \\ EXPERIMENTAL CONICAL NOZZLE HELIOS-X
}

\author{
San Luis B. Tolentino Masgo ${ }^{1,2, *}$, Richard Nakka ${ }^{3}$, Simón Caraballo ${ }^{4}$, Jorge Mírez ${ }^{2,5}$
}

Recibido: 11-03-2020, Revisado: 11-03-2020, Aprobado tras revisión: 17-10-2020

\section{Resumen}

Estudios numéricos del campo de flujo para toberas convergentes-divergentes con longitud de garganta, han reportado fluctuaciones del flujo con ondas de choque oblicuo en la sección de la garganta, para la condición de flujo sobre-expandido. Sin embargo, para otras condiciones del flujo, para un mismo tipo de tobera, el conocimiento es limitado. En el presente trabajo, el objetivo es determinar el comportamiento del flujo en la longitud de garganta y en la divergente, para una tobera cónica experimental clasificada como Helios-X, para la condición de flujo sub-expandido. Se realizaron simulaciones numéricas $2 \mathrm{D}$ del campo de flujo con el código ANSYS-Fluent versión 12.1, aplicando el modelo RANS. Se emplearon las ecuaciones gobernantes para el flujo compresible, conservación de la masa, cantidad de movimiento, energía y de estado; así como, para la turbulencia el modelo SST $k-\omega$ de Menter y para la viscosidad en función de la temperatura la ecuación de Sutherland.

\begin{abstract}
Numerical studies of the flow field for convergentdivergent nozzles with throat length, have reported fluctuations of the flow with oblique shock waves in the throat section, for the over-expanded flow condition. However, for other flow conditions, for the same type of nozzle, knowledge is limited. In the present work, the objective is to determine the behavior of the flow in the throat length and in the divergent, for an experimental conical nozzle classified as Helios-X, for the under-expanded flow condition. 2D numerical simulations of the flow field were performed with the ANSYS-Fluent version 12.1 code, applying the RANS model. The governing equations for compressible flow, conservation of mass, momentum, energy, and state were used; as well as, for turbulence, the Menter model SST $k-\omega$ and for the viscosity as a function of temperature the Sutherland equation.
\end{abstract}

\footnotetext{
${ }^{1}$ Universidad Nacional Experimental Politécnica Antonio José de Sucre, Vice-Rectorado Puerto Ordaz, Venezuela.

${ }^{2, *}$ Grupo de Modelamiento Matemático y Simulación Numérica, Universidad Nacional de Ingeniería, Lima, Perú. Autor para correspondencia : sanluist@gmail.com. (D) http://orcid.org/0000-0001-6320-6864,

${ }^{3}$ Cohetería experimental amateur, Canadá. (D) http://orcid.org/0000-0002-7759-7162

${ }^{4}$ Departamento de Ingeniería Mecánica, Universidad Nacional Experimental Politécnica Antonio José de Sucre Vice-Rectorado Puerto Ordaz, Bolívar, Venezuela. (D) http://orcid.org/0000-0002-0170-2448

${ }^{5}$ Facultad de Ingeniería de Petróleo Gas y Petroquímica, Universidad Nacional de Ingeniería, Lima, Perú.

(D) http://orcid.org/0000-0002-5614-5853
}

Forma sugerida de citación: Tolentino Masgo, S. L. B.; Nakka, R.; Caraballo, S. y Mírez, J. (2021). «Simulación numérica del flujo subexpandido en la tobera cónica experimental helios-x». INGENIUS. N. ${ }^{\circ} 25$, (enero-junio). pp. 81-93. DOI: https://doi.org/10.17163/ings.n25.2021.08. 
En la sección de la garganta, adyacente a la pared, el flujo presentó fluctuaciones, en la simetría axial el flujo presentó una aceleración escalonada; en la sección divergente, el flujo se desaceleró en cierta región, sin embargo, el flujo salió de la tobera a velocidad supersónica ligeramente mayor de Mach 3. Se concluye que en la sección de la longitud de garganta se presenta un patrón de flujo, así como, en la sección divergente.

Palabras clave: garganta, fluctuación, subexpandido, simulación, tobera
In the section of the throat, adjacent to the wall, the flow presented fluctuations, in the axial symmetry the flow presented a stepped acceleration; in the divergent section, the flow slowed in a certain region, however, the flow exited the nozzle at a supersonic speed slightly greater than Mach 3 . It is concluded that in the throat length section there is a flow pattern, as well as, in the divergent section.

Keywords: Throat, Fluctuation, Under-expanded, Simulation, Nozzle. 


\section{Introducción}

El flujo en toberas de motores de cohetes supersónicos es estudiado de manera recurrente para diferentes configuraciones geométricas del perfil interno de las paredes de la tobera. La sección divergente de la tobera puede ser de geometría cónica, de campana, parabólica, rectangulares, planas, entre otras. Tomar datos experimentales en un banco de prueba en estática del flujo dentro de la tobera tiene limitaciones, cuando el flujo de gas está muy por arriba de la temperatura del ambiente. Por lo cual, durante el ensayo experimental registran los datos con instrumentos sensibles a los cambios de presión, temperatura, vibraciones, en la cámara de combustión, al inicio de la tobera, en las paredes, y mediante capturas de imágenes de la región del flujo que se descarga al ambiente. Cuando el flujo está estrangulado en la garganta y basándose a la presión de la cámara de combustión, el flujo puede estar sobreexpandido, óptimamenteexpandido o subexpandido [1]. Señalando que, para un flujo sobreexpandido el choque se presenta dentro de la tobera y para un flujo subexpandido el choque se presenta fuera de ella.

Los datos experimentales obtenidos en la frontera de la tobera, son utilizados para reproducir el comportamiento del campo de flujo dentro de la tobera, mediante la aplicación de la dinámica de fluidos computacional (CFD, por sus siglas en inglés) [2,3], el cual permite cuantificar las magnitudes termodinámicas en un dominio computacional del campo de flujo que no es posible obtener por medios experimentales en campo abierto y laboratorios.

La CFD es una herramienta computacional que permite obtener soluciones aproximadas de la realidad del fenómeno físico, si de manera adecuada se toma en cuenta la geometría del dominio computacional, el refinamiento de la malla, el modelo de turbulencia, el establecimiento de los errores durante la etapa de iteración, así como, otros parámetros de control de variables.

Para flujo compresible están sustentados y reportados trabajos sobre las ondas de choque y ondas de expansión de Prandtl-Meyer [4,5], la captura de imagen de las ondas de choque mediante la técnica Schlieren [6], la turbulencia [7], la capa límite [8,9], así como los modelos de turbulencia [10].

Estudios experimentales en estática y en dinámica para toberas convergentes-divergentes con longitud de garganta han sido reportados con fines de investigación, sin embargo, el conocimiento del comportamiento del régimen del flujo que se presenta dentro de dicha toberas con longitud de garganta es prácticamente nulo, ya que escasamente ha sido abordado empleando herramientas computacionales para determinar sus magnitudes termodinámicas.

Un estudio computacional de la simulación del flujo sobreexpandido en una tobera cónica con longitud de garganta y ángulo medio de $11^{\circ}$ en la sección divergente, perteneciente a un motor de cohete sonda para combustible sólido, clasificado como ULA-1A XP [11], reportó resultados numéricos de las fluctuaciones de la velocidad del flujo y de los choques oblicuos que se presentaron en la sección de la garganta, en el rango de velocidad transónica, así como el comportamiento de la fluctuación del flujo desde el centro hacia las paredes de la garganta. Acotando que, previamente, este tipo de tobera fue ensayada en estática y en dinámica por el Grupo de Ciencias de la Atmósfera y del Espacio (GCAE), de la Universidad de Los Andes, Venezuela [12-14].

En otro estudio realizado, para un flujo subexpandido en una tobera cónica con longitud de garganta y ángulo medio de $15^{\circ}$ en la sección divergente, identificada como tobera Helios-X [15], que también fue simulado el campo de flujo, expusieron sus resultados según el número de Mach, donde, el flujo en la sección de la garganta presentó desaceleración. Aunque aportan un dato fundamental de la velocidad del flujo de acuerdo con el número de Mach, aún queda por analizar con mayor detalle, el campo de flujo para otros parámetros termodinámicos. Por lo cual, es de interés que se debe continuar con la investigación para este tipo de tobera, para determinar el comportamiento del campo de densidad, presión, temperatura, velocidad, asimismo el número de Mach, en todo el dominio, y enfocando con mayor interés en la sección de la garganta, que podrían aportar y enriquecer el conocimiento de la distribución de los gradientes de los parámetros termodinámicos.

La tobera Helios-X, diseñada por Nakka [16], fue ensayada en un banco de prueba en estática y en dinámica. En la Figura 1 se muestra el registro del ensayo en estática de la tobera acoplada al motor cohete Helios-X, el cohete en la plataforma de lanzamiento y el despegue del cohete. Múltiples experimentos realizados por Nakka de toberas en motores de cohetes para combustible sólido de categoría aficionado, en estática y en dinámica, pueden ser obtenidos en su sitio web [16].

En el presente trabajo se ha propuesto continuar con la investigación para el flujo subexpandido en la tobera Helios-X, reportado en [15], con el fin de determinar el comportamiento del flujo en la sección de la garganta y en la divergente. Para lograr el objetivo propuesto, se simuló el campo de flujo supersónico y se obtuvo el campo de presión, número de Mach, velocidad, temperatura y densidad. Los resultados muestran, en la pared, a la entrada de la garganta, una región del flujo que presenta fluctuación, por lo cual, en esa región el flujo acelera y desacelera, en otras regiones, de la misma sección, la velocidad del flujo solo se lentifica.

En la sección 2 se presentan las ecuaciones empleadas, el dominio computacional 2D y el método de solución computacional. En la sección 3 se exponen los 
resultados obtenidos y las discusiones. En la sección 4 se exponen las conclusiones del análisis realizado.

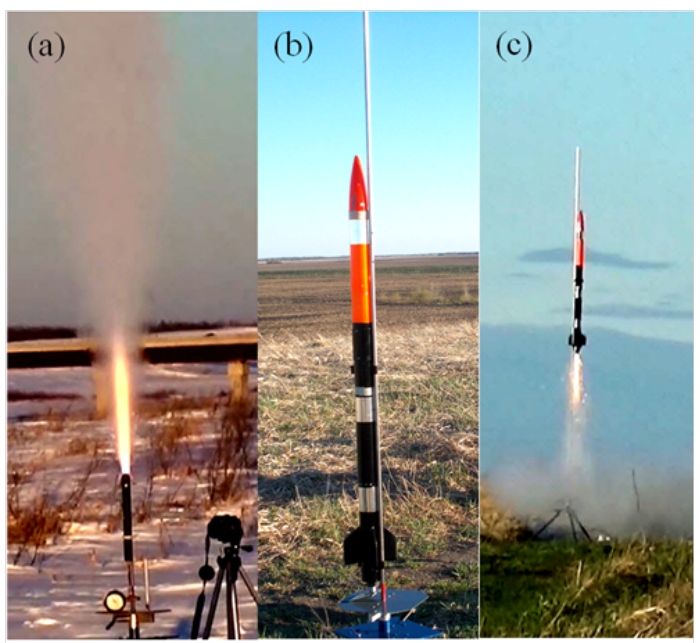

Figura 1. (a) Ensayo experimental en estática de la tobera con longitud de garganta acoplada al motor cohete Helios-X, marzo 2017. (b) Plataforma de lanzamiento del cohete Z-30, con el motor cohete Helios-X instalado. (c) Despegue del cohete Z-30, mayo 2017 [16]

\section{Materiales y métodos}

\subsection{Fundamento matemático}

En el presente trabajo, para la simulación del campo de flujo compresible, se emplea el modelo de las ecuaciones de Navier-Stokes de número de Reynolds promedio (RANS, por sus siglas en inglés). Las ecuaciones gobernantes empleadas son: la ecuación de la conservación de la masa (1), cantidad de movimiento (2), conservación de la energía (3) y de estado (4). En forma compacta y para un flujo en condiciones estacionarias, se expresan como:

$$
\nabla \cdot\left(\rho u_{i}\right)=0
$$

Donde, la densidad es $\rho$ y la velocidad $u$.

$$
\nabla \cdot\left(\rho u_{i} u_{j}\right)=-\nabla p+\nabla(\overline{\bar{\tau}})+\nabla \cdot\left(-\rho \overline{u_{i} u_{j}}\right)
$$

Donde, la presión es $p$; el tensor de tensiones $\overline{\bar{\tau}}$ y las tensiones de Reynolds $-\rho \overline{u_{i} u_{j}}$. Cabe señalar, la ecuación (2) está cerrada, ya que incluye el término de tensiones de Reynolds.

$$
\nabla \cdot\left(u_{i}(\rho E+p)\right)=\nabla \cdot\left(k_{e f f} \nabla T+\left(\overline{\bar{\tau}}_{e f f} \cdot u_{i}\right)\right)
$$

Donde, la energía total es $E$, la temperatura $T$, la conductividad térmica efectiva $k_{e f f}$, y el tensor de tensiones efectivo $\overline{\bar{\tau}}_{e f f}$.

$$
p=\rho R T
$$

Donde, la constante del gas es $R$.

Para flujo compresible, se toman en cuenta las relaciones de presiones (5) y de temperaturas (6) en función del número de Mach el cual es el parámetro dominante, y se expresan como:

$$
\begin{gathered}
\frac{p_{0}}{p}=\left(1+\frac{\gamma-1}{2} M^{2}\right)^{\frac{\gamma}{\gamma-1}} \\
\frac{T_{0}}{T}=1+\frac{\gamma-1}{2} M^{2}
\end{gathered}
$$

Donde, la presión total es $p_{0}$, la temperatura total $T_{0}$, la relación de calores específicos $\gamma$ y el número de Mach, $M$.

El número de Mach supersónico en la salida de la tobera para un flujo subexpansionado y sin choque en la salida de la divergente se determina con la ecuación (7); donde $A / A^{*}$ es la relación de áreas de diseño, el área en la salida de la tobera es $A$ y de la garganta $A^{*}$ :

$$
\frac{A}{A^{*}}=\frac{1}{M}\left(\frac{1+\frac{\gamma-1}{2} M^{2}}{\frac{\gamma+1}{2}}\right)^{\frac{\gamma+1}{2 \gamma-2}}
$$

Las consideraciones del número de Mach son las siguientes: para flujo incompresible $M<0,3$; flujo subsónico $0,3<M<0,8$; flujo transónico $0,8<$ $M<1,2$; flujo supersónico $1,2<M<5$; flujo hipersónico $M>5$; y para el flujo con velocidad sónica, se tiene $M=1$ [5]. Cabe señalar, White [5] considera que a partir de un valor mayor de Mach 3 el flujo es hipersónico, y Anderson [4] a partir de Mach 5.

Para la viscosidad en función de la temperatura (8), de acuerdo con la ley de Sutherland [8], se expresa como:

$$
\frac{\mu}{\mu_{0}}=\left(\frac{T}{T_{0}}\right)^{\frac{3}{2}} \frac{T_{0}+S}{T+S}
$$

Donde, la viscosidad de referencia es $\mu_{0}=$ $1,716 \mathrm{~kg} /(\mathrm{m} \cdot \mathrm{s})$, la temperatura de referencia $T_{0}=$ $273,11 \mathrm{~K}$ y la temperatura efectiva $S=110,56 \mathrm{~K}$.

Para la turbulencia del flujo, se toma en cuenta el modelo de turbulencia SST $k-\omega$ de Menter [17], la cual es resuelta en conjunto con la ecuación de cantidad de movimiento. Este modelo de turbulencia contiene dos ecuaciones, una para la energía cinética específica $k$, y la otra para la tasa de disipación específica $\omega$, por lo cual, logra mejorar las respuestas en presencia de gradientes adversos de presión, y separación de flujo.

El modelo de turbulencia de Menter [17] ha sido comparado con otros modelos de turbulencia para diferentes condiciones del flujo compresible con presencia 
de ondas de choque en diferentes equipos experimentales para flujo sobreexpandido y flujo subexpandido, donde los resultados numéricos para dominios $2 \mathrm{D}$ de geometrías simétricas se superponen sobre los datos experimentales de presión en las paredes de equipos experimentales, en una tobera $[18,19]$ y en un difusor transónico [20,21], siendo las formas de las ondas de choque de los resultados numéricos aproximadamente similares con las experimentales. Aplicaciones del modelo de turbulencia de Menter sustentan su validez para diferentes condiciones del régimen del flujo [22-25]. En dominios 3D, el aporte del análisis físico es mucho mayor, incluso para geometrías asimétricas, y cargas laterales asimétricas en el campo de flujo. Por tanto, para las condiciones del flujo subexpandido y sin condiciones de cargas laterales asimétricas, el modelo de turbulencia de Menter es adecuado para la simulación 2D del flujo subexpandido del presente trabajo.

\subsection{Dominio computacional}

El esquema de la geometría de la tobera cónica experimental convergente-divergente con longitud de garganta, Helios-X [16], se muestra en la Figura 2, y cuyas dimensiones principales y sus respectivas unidades en milímetros se muestran en la misma figura. Siendo longitud de garganta $L_{g}=6,01 \mathrm{~mm}$, diámetro de la garganta $D_{g}=5,58 \mathrm{~mm}$, la relación entre la longitud de garganta y el diámetro $r_{L D}=1,07$, y la relación de áreas de diseño $A / A^{*}=5,206$.

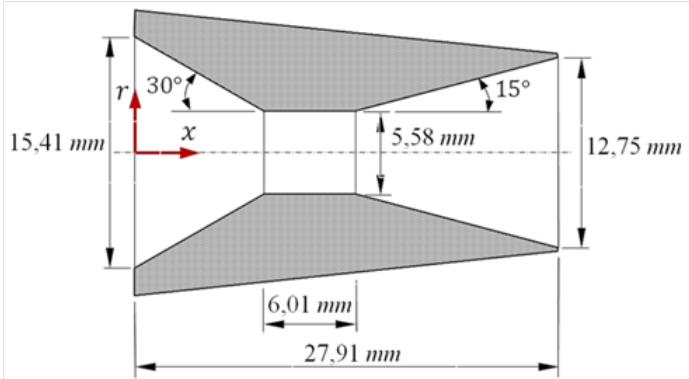

Figura 2. Geometría de la tobera cónica experimental Helios-X [16]

El dominio computacional 2D con simetría axial compuesto por una sección de la cámara de combustión, la tobera y la atmósfera, se muestra en la Figura 3. El propósito de tomar en cuenta una sección corta de la cámara de combustión de 7,47 $\mathrm{mm}$ y no toda la sección del tubo motor, es para aplicar la carga de presión y direccionar el flujo hacia la entrada de la tobera. La longitud de la sección de la tobera cónica es 27,91 $\mathrm{mm}$ y la longitud de la sección del ambiente de la atmósfera $281 \mathrm{~mm}$, respectivamente. Señalando que, la garganta inicia en la posición $x=8,51 \mathrm{~mm}$ y termina en la posición $x=14,53 \mathrm{~mm}$, y su longitud es $L_{g}=6,01 \mathrm{~mm}$.

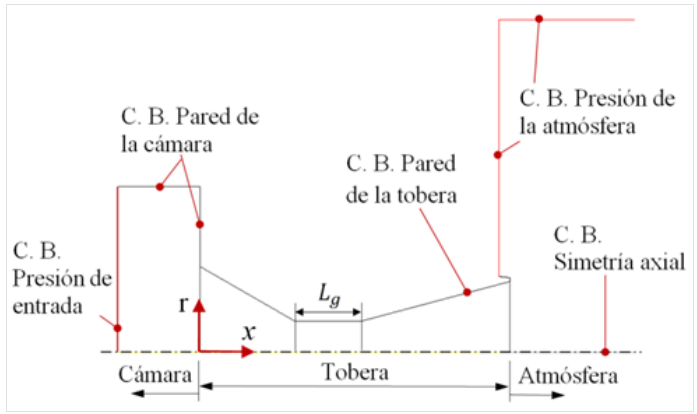

Figura 3. Dominio computacional 2D con simetría axial. El dominio incluye una sección de la cámara de combustión, la tobera y una sección de atmósfera

Además, como la tobera tiene una geometría simétrica de revolución, un dominio 2D es adecuado, lo cual contribuye en la reducción de la cantidad de celdas de la malla y el tiempo de iteración durante el procesamiento de datos computacionales. Además, en la misma figura del dominio se señalan las referencias donde se aplican las condiciones de borde (C.B.).

Las condiciones iniciales y de borde se establecieron como:

En la cámara del motor cohete Helios-X, la presión total absoluta se establece 6996,11 kPa; y de la temperatura total $2558 \mathrm{~K}$.

En el ambiente de la atmósfera, la presión se establece $101,5 k P a$, de la temperatura $263,15 \mathrm{~K}$.

En el eje de simetría axial, la velocidad del flujo en la dirección radial es nula. En las paredes la velocidad es nula por la condición de no deslizamiento.

Las paredes de la sección de la cámara de combustión y de la tobera son consideradas adiabáticas.

El efecto de la gravedad del flujo dentro de la tobera no se considera, esto es debido a la alta velocidad del chorro supersónico en la divergente. En la atmósfera, la velocidad del flujo es supersónico, por lo cual, para la longitud del dominio considerado, el efecto de la gravedad es ínfimo y es considerado despreciable.

Cabe señalar, los datos de presión y de temperatura en la cámara del motor cohete que se aplican en las condiciones de borde del presente trabajo han sido obtenidos por medios experimentales por Nakka [16], así como, los datos del ambiente de la atmósfera registrados con instrumentos de medición de presión y temperatura. Los datos experimentales de la cámara de combustión y del ambiente de la atmósfera aplicados al dominio 2D, contribuyen para obtener la simulación del campo de flujo en todo el dominio, y permiten 
determinar el comportamiento de la presión y temperatura en la pared de la tobera que no ha sido posible medir experimentalmente.

Para el presente trabajo, en las simulaciones numéricas para el presente trabajo, los gases quemados producto de la combustión del propelente sólido compuesto por nitrato de amonio, aluminio, azufre y cloropreno (A24 ANCP) [16], se considera como gas ideal, y como sustancia aire. Como parámetros, se fija la relación de calor específico $\gamma=1,4$, la constante del gas $R=287 \mathrm{~J} /(\mathrm{kg} \cdot K)$, el calor específico a presión constante $C_{p}=1006,43 \mathrm{~J} /(\mathrm{kg} \cdot \mathrm{K})$ y la conductividad térmica $k=0,0242 \mathrm{~W} /(m \cdot K)$.

El dominio computacional mallado se muestra en la Figura 4 y una ampliación de la sección mallada de la tobera se muestra en la misma figura. El mallado se realizó en la plataforma ANSYS-Meshing y se discretizó el dominio mediante la interacción de ICEM-CFD. Se refinó la malla del dominio a lo largo de todas las paredes, por la presencia del esfuerzo cortante en esas regiones. En la sección de la cámara y de la tobera se aplicó un mallado con celdas triangulares, y en la sección del ambiente de la atmósfera un mallado estructurado con celdas cuadriláteras, para un total de 32675 de celdas combinadas. El mallado del dominio mostrado en la figura corresponde para un mallado final, luego de haber realizado un estudio de convergencia numérica para un refinamiento del dominio tres veces.
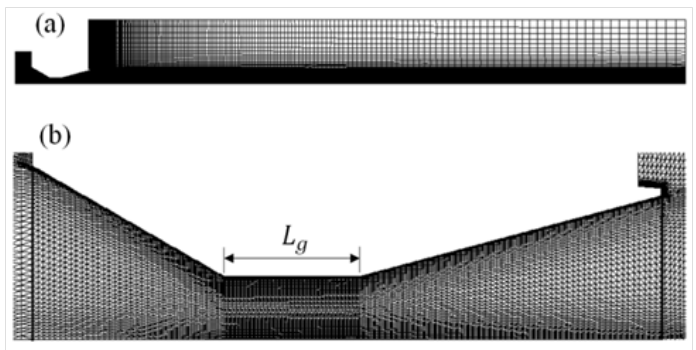

Figura 4. (a) Dominio computacional mallado con un total de 32675 celdas combinadas. (b) Sección del dominio mallado de la tobera.

En el estudio de convergencia numérica, el primer dominio mallado arrojó 30950 celdas, siendo la celda de menor dimensión de $4,9 \times 10^{-5} \mathrm{~mm}$ y de mayor dimensión de $3,37 \times 10^{-4} \mathrm{~mm}$ en la sección de la tobera, y la mayor dimensión de la celda de $3,2 \mathrm{~mm}$ se presentó en la esquina superior derecha en el dominio de la atmósfera. El segundo arrojó 32 296, siendo la celda de menor tamaño de $4,7 \times 10^{-5} \mathrm{~mm}$ y la máxima de $3,31 \times 10^{-4} \mathrm{~mm}$, y en el dominio de la atmósfera la celda máxima de $3,2 \mathrm{~mm}$. El tercero arrojó 32 675 celdas, y con dimensiones de celdas mínima de $4,67 \times 10^{-5} \mathrm{~mm}$ y máxima de $3,29 \times 10^{-4} \mathrm{~mm}$, y en la atmósfera la celda de mayor dimensión de $3,2 \mathrm{~mm}$. Para el primer dominio, el espaciamiento mínimo de la celda en la pared de la tobera, en promedio se obtuvo $y^{+}=0,98$, para el segundo dominio $y^{+}=0,95$ y para el tercer dominio del mallado final $y^{+}=0,94$.

Para los tres casos, la densidad de la malla es alta en la tobera y en la región de la atmósfera donde se presenta el chorro supersónico conocido como pluma. Se tomó como punto de control al final de la sección divergente, en la simetría axial, para evaluar numéricamente el número de Mach, por ser una región crítica por la alta velocidad del flujo, y se obtuvo un error porcentual de 0,04\% entre el tercer dominio mallado y el segundo; y ligeramente mayor, de 0,052 \% entre el segundo y el primero; siendo en la salida de la tobera en la simetría axial del eje $\mathrm{X}$ para el tercer dominio de mallado final y el valor numérico de Mach 3,1.

Para la teoría de flujo cuasiunidimensional con $\gamma=1,4$, y $A / A^{*}=5,206$ de diseño de la tobera Helios-X, y sin choque a la salida de la tobera, se tiene el valor de Mach 3,217 obtenido con la Ecuación (7) (teórica). Siendo la diferencia de magnitud de número de Mach 0,117 entre el cálculo teórico de Mach 3,217 y el cálculo numérico de Mach 3,1 del tercer dominio mallado. Por lo cual, es aceptable la magnitud del resultado de las comparaciones de números de Mach como validación numérica. Para un flujo cuasiunidimensional y subexpandido, el flujo sale de la tobera de manera uniforme y perpendicular al área de salida, por lo cual tiene la misma magnitud en su área de sección transversal, mientras que, para el método numérico el flujo tiene un gradiente de velocidad del número de Mach a la salida de la tobera y su magnitud varía en su área de sección transversal.

El dominio mallado que se presenta en la Figura 4 es adecuado y cumple con los criterios satisfactorios del análisis de convergencia realizado. Aunque los tres dominios satisfacen para $y^{+}<1$, se optó por el dominio mallado con 32675 celdas por tener un mayor refinamiento en las paredes, la cual es empleado en las simulaciones computacionales.

La calidad de la malla, para celdas bidimensionales, y el sesgo equiángulo $\left(Q_{E A S}\right)$, establecen que debe estar $0 \geq Q_{E A S} \geq 1$, para cualquier celda 2D [26]. Para todo el dominio de la malla final, se obtuvo $Q_{E A S}=0,55$. Acotando que, el estudio de convergencia numérica se realizó con el modelo de turbulencia SST $k-\omega$ de Menter [17] para simular la turbulencia del flujo.

\subsection{Método de solución computacional}

Para la simulación del flujo, en el código ANSYSFluent 12.1, el cual aplica el método de volumen finito (MVF), se optó la opción de análisis basado en densidad para un fluido compresible, dominio $2 \mathrm{D}$ con simetría axial en el eje x. Para la turbulencia del flujo se empleó el modelo de SST $k-\omega$ de Menter [17] y para la viscosidad la ecuación de Sutherland [8]. 
En el método de solución, se consideró, la formulación implícita y tipo de flujo Roe-FDS. Para la discretización espacial, el gradiente: Least Squares Cell based; para el flujo, energía cinética turbulenta y la tasa de disipación específica, la opción: First Order Upwin.

En el monitor residual, para el criterio de convergencia absoluta, se estableció un valor fijo de 0,00001, tanto para continuidad, velocidad, y energía. Se realizaron 136400 iteraciones en un tiempo cercano a cincuenta horas, para obtener la convergencia numérica de los resultados finales del campo de flujo, de número de Mach, presión, velocidad, temperatura y densidad.

Para el procesamiento de datos se empleó un equipo con las siguientes características: Laptop marca Síragon, modelo M54R, Intel Core 2 Duo, dos procesadores de 1,8 GHz y memoria RAM de 3 GB.

\section{Resultados y discusión}

En esta sección se presentan los resultados del campo de flujo, para la carga aplicada de presión de entrada de $6996,11 \mathrm{kPa}$ y temperatura de $2558 \mathrm{~K}$, presión del ambiente en la salida de la tobera $101,5 k P a$ y temperatura de $263,15 \mathrm{~K}$.

Las variaciones de las magnitudes de la presión estática (Figura 5), de número de Mach (Figura 6), de velocidad (Figura 7), de temperatura estática (Figura 8) y de la densidad (Figura 9), muestran en qué regiones del dominio alcanzan valores máximos y mínimos. Mediante las líneas de contorno se observa de qué manera se distribuyen en diferentes regiones del campo de flujo, en la sección de la tobera así como en la sección de la atmósfera.

El flujo que sale de la tobera está subexpandido, y se muestra en la atmósfera cómo están conformadas las ondas de choque; y esa región del chorro supersónico donde se presentan las ondas, en la literatura es conocida como pluma.

En la Figura 10 se muestra los perfiles evaluados en la simetría axial, para la región del flujo supersónico que sale de la tobera y descarga en la atmósfera. Se muestran las fluctuaciones producto de la onda de choque, incluso en ciertas regiones la presión cae por debajo de la presión de la atmósfera, acelerando el flujo en esa región antes que se presente el choque a un valor cercano Mach 4,75, y velocidad de $2050 \mathrm{~m} / \mathrm{s}$; mientras que la temperatura cae por debajo de $500 \mathrm{~K}$. Después del choque, el flujo sigue siendo supersónico con presencia de fluctuaciones amortiguadas alrededor de Mach 3, velocidad de $1800 \mathrm{~m} / \mathrm{s}$ y temperatura de $900 \mathrm{~K}$. Además, el comportamiento de las curvas de presión y de densidad muestra una tendencia de equilibrio.

Se han considerado las líneas de contorno, en la sección de la tobera, las cuales aporta mayor infor- mación en cuanto a la distribución de los gradientes de los parámetros termodinámicos. En las figuras ampliadas se ilustra cómo se distribuyen las líneas de contorno en las secciones convergente, de la garganta de longitud $L_{g}$ y en la divergente: para la presión estática (Figura 11), para el número de Mach (Figura 12), para la velocidad (Figura 13), para la temperatura estática (Figura 14) y para la densidad en la (Figura 15). Se observa que las líneas de contorno al inicio de la sección de la garganta tiene un comportamiento distinto a las líneas de contorno al final de la misma garganta, así como, en la parte media.

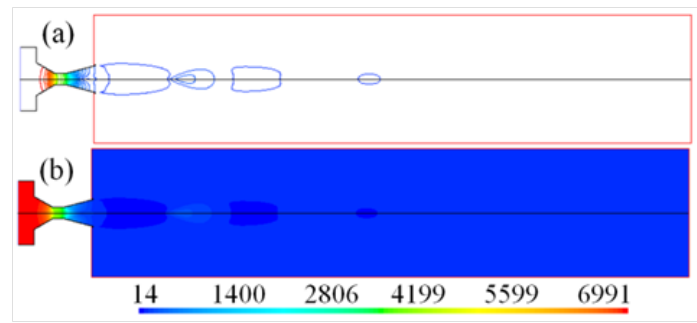

Figura 5. Campo de presión estática $(\mathrm{kPa})$

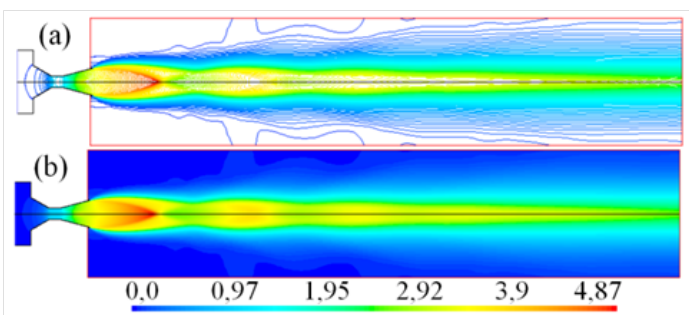

Figura 6. Campo de número de Mach

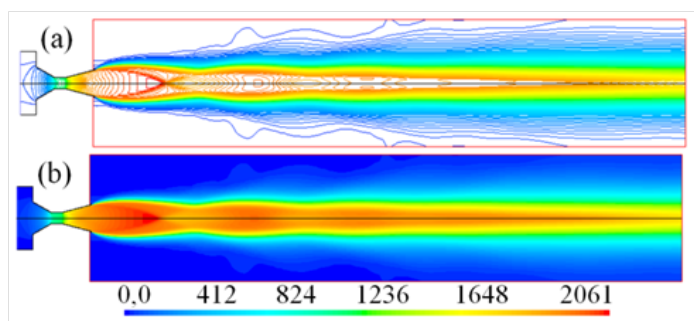

Figura 7. Campo de velocidad (m/s)

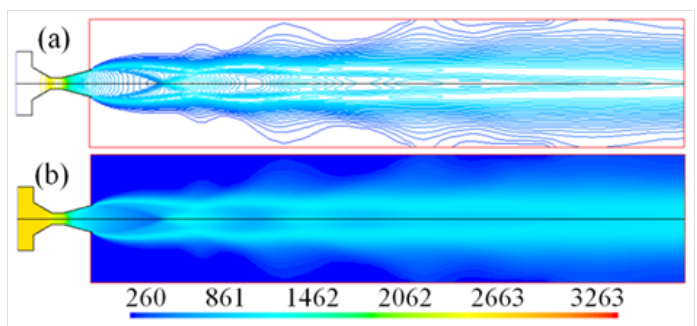

Figura 8. Campo de temperatura estática (K) 


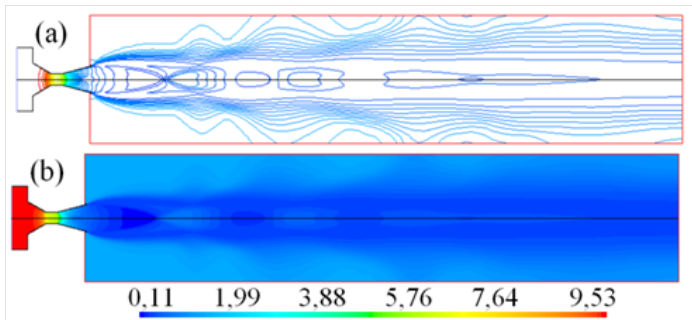

Figura 9. Campo de densidad $\left(\mathrm{kg} / \mathrm{m}^{3}\right)$.

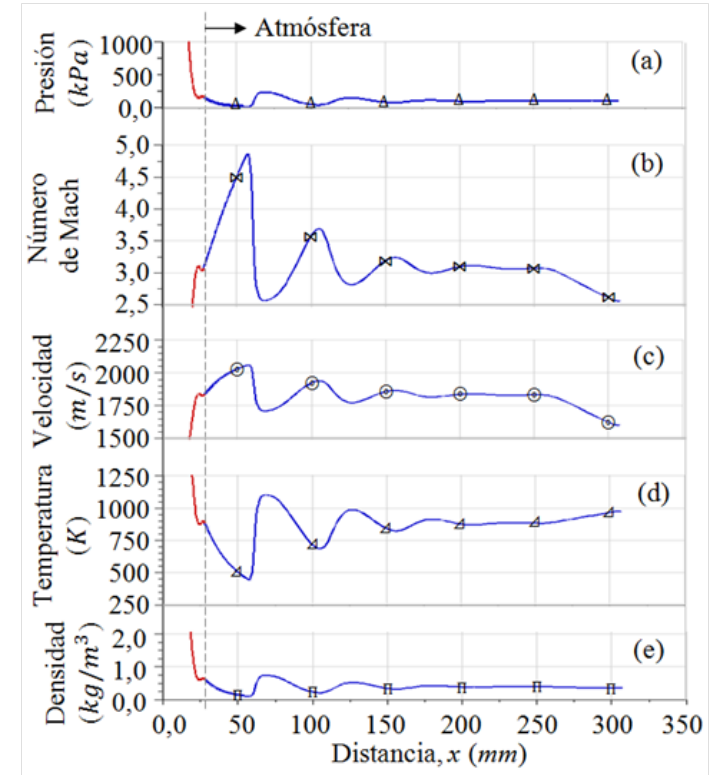

Figura 10. Perfiles evaluados en la simetría axial, en el eje $\mathrm{X}$, en la región del ambiente de la atmósfera. (a) Presión. (b) Número de Mach. (c) Velocidad. (d) Temperatura y (e) Densidad

Cabe señalar, para el caso de la presión estática, se observa que en la parte media de la longitud de garganta, las líneas de contorno tienen una tendencia a ser perpendicular a las paredes de la garganta, por lo cual, la magnitud de la presión en la simetría es similar a la magnitud de la presión en la pared.

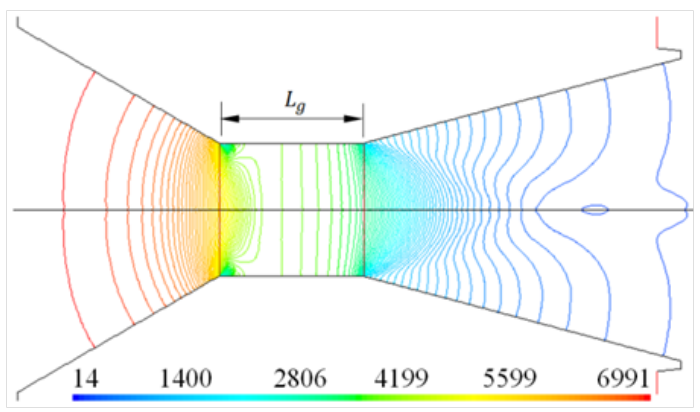

Figura 11. Líneas de contorno de presión estática $(\mathrm{kPa})$ del flujo en la sección de la tobera

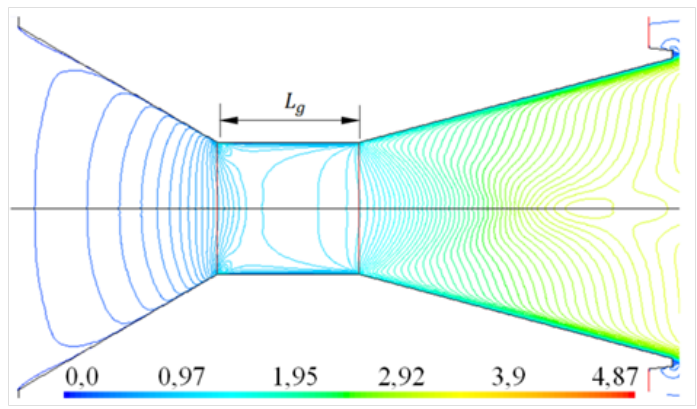

Figura 12. Líneas de contorno de número de Mach del flujo en la sección de la tobera

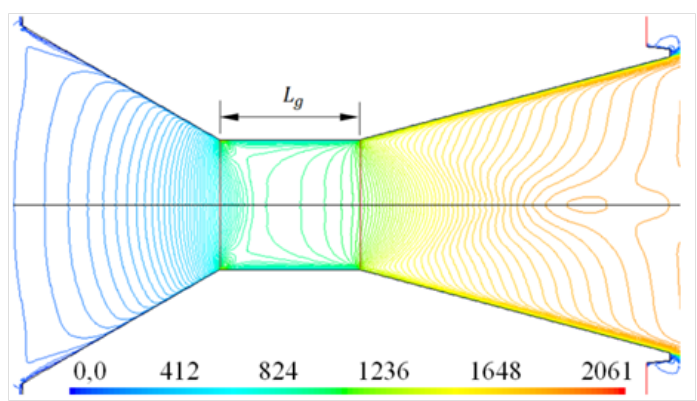

Figura 13. Líneas de contorno de velocidad $(\mathrm{m} / \mathrm{s})$ del flujo en la sección de la tobera

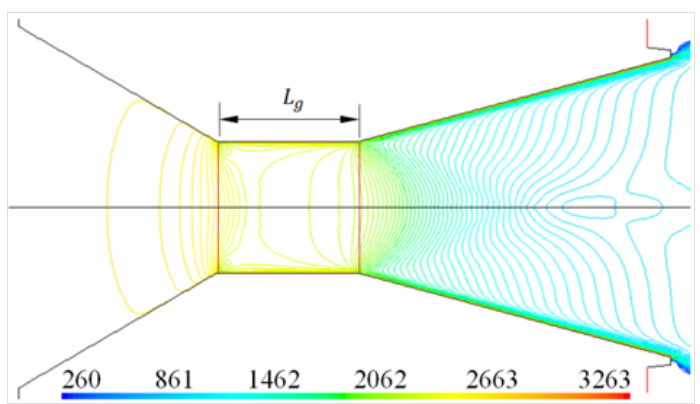

Figura 14. Líneas de contorno de temperatura estática (K) del flujo en la sección de la tobera

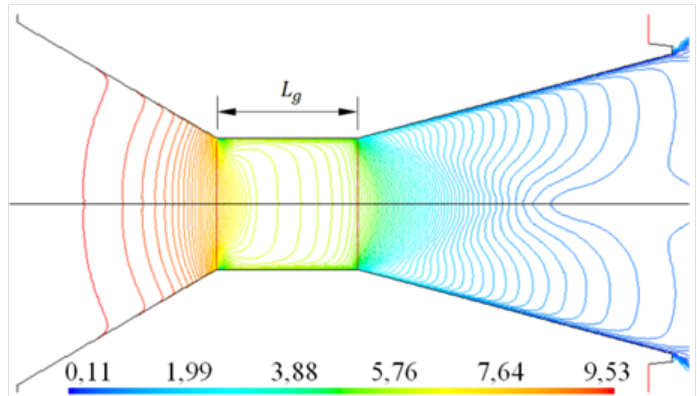

Figura 15. Líneas de contorno de densidad $\left(\mathrm{kg} / \mathrm{m}^{3}\right)$ del flujo en la sección de la tobera

Analizando el comportamiento del flujo desde otra perspectiva, a través de las trayectorias de las curvas numéricas, se muestra que el cambio brusco de la caída 
de presión estática (Figura 16) se presenta en el primer vértice de la sección de la garganta. En la sección media de la garganta, un tramo de las trayectorias de los perfiles de presión en la pared y en la simetría axial es coincidente, lo cual indica que se presenta un régimen de flujo uniforme, con incidencias en la aceleración del flujo. Aguas abajo, a partir de la posición $x=22,5 \mathrm{~mm}$, la caída de presión del flujo tiende a frenar, incrementa ligeramente, después disminuye con menor intensidad, siendo un tramo de la trayectoria del perfil con tendencia oscilatoria; en dicho tramo, las fluctuaciones de presión influyen en el desarrollo de la aceleración del flujo, por lo cual, las magnitudes de los parámetros termodinámicos presentan variaciones.

Para el caso del número de Mach (Figura 17), se muestra la desaceleración de flujo en la sección de la garganta, siendo en la parte media de la sección de la garganta Mach 1, y en la salida de la garganta mayor a este valor. Cerca de la salida de la sección divergente, la velocidad del flujo alcanza un valor mayor de Mach 3, en la posición $x=22,5 \mathrm{~mm}$, aguas abajo se presenta una curvatura, y tiende a disminuir el flujo hasta la salida de la tobera, manteniendo su velocidad mayor a Mach 3, siendo el flujo supersónico. Desde la posición $x=22,5 \mathrm{~mm}$ hasta la salida de la tobera, el comportamiento de la trayectoria de la curva muestra que se produce una desaceleración del flujo antes que salga de la tobera.

En el perfil de velocidad (Figura 18) se observa cómo se comporta el patrón de velocidad en la simetría axial, mas no se presenta en la pared por ser nula la velocidad allí por la condición de no deslizamiento. En la parte media de la sección de la garganta el flujo tiene una velocidad estimada de $920 \mathrm{~m} / \mathrm{s}$; aguas abajo, se muestra cómo se comporta un tramo de la trayectoria del perfil de la velocidad a partir de la posición $x=22,5 \mathrm{~mm}$, con una tendencia de velocidad ligeramente mayor de $1800 \mathrm{~m} / \mathrm{s}$. También como en el caso anterior, para el número de Mach, la velocidad del flujo se desacelera antes de salir de la tobera.

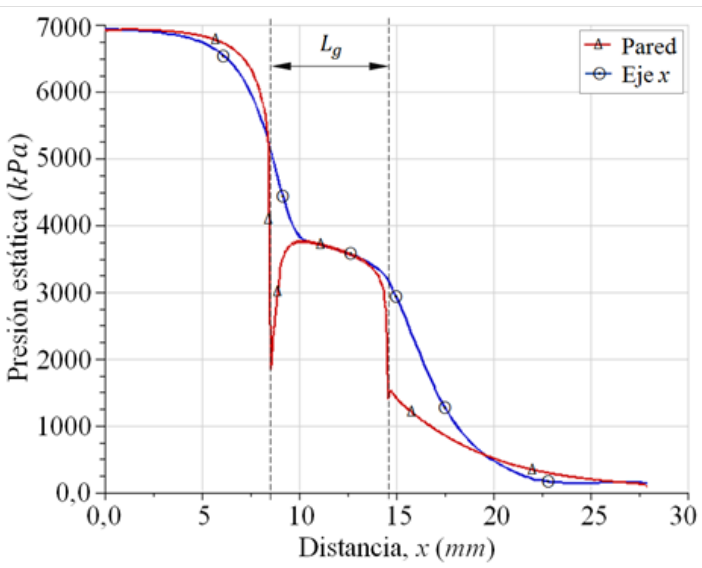

Figura 16. Perfiles de presión estática evaluados en la pared y en el eje X, en la sección de la tobera

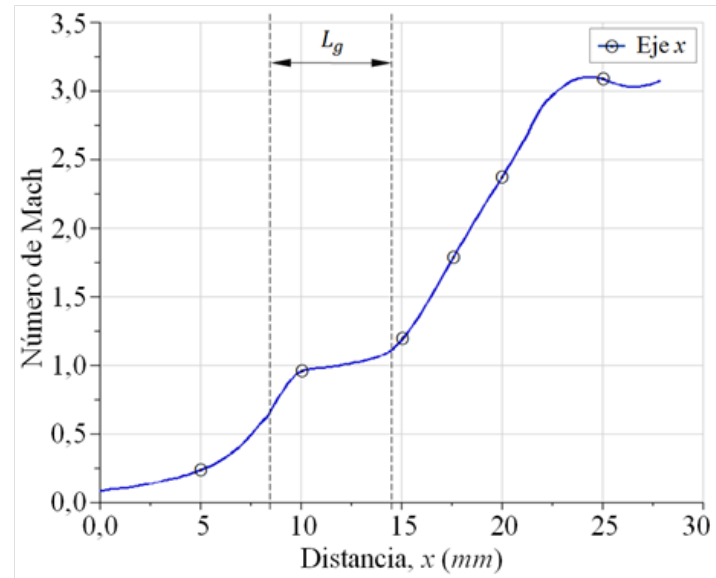

Figura 17. Perfil de número de Mach evaluado en el eje $\mathrm{X}$, en la sección de la tobera

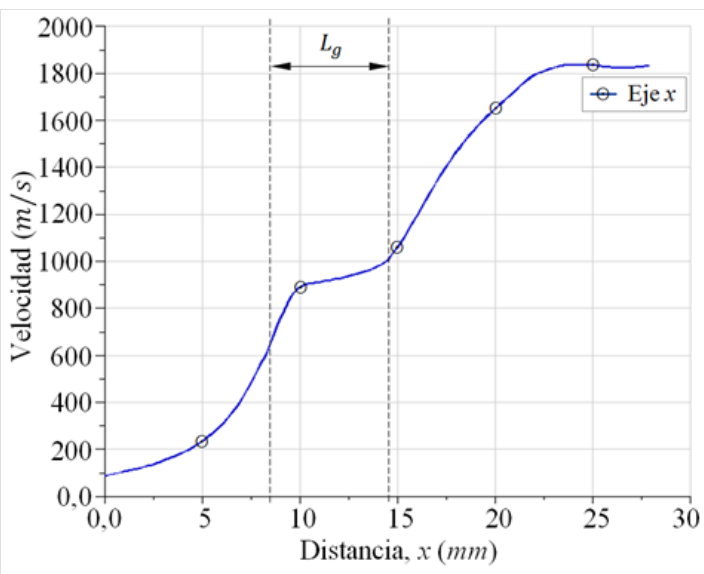

Figura 18. Perfil de velocidad evaluado en el eje X, en la sección de la tobera

Asimismo, para los perfiles de temperatura estática (Figura 19), se muestra el comportamiento de las trayectorias en la pared y en la simetría axial. En la sección de la garganta se observa que la temperatura aumenta y disminuye, e influyen los vértices a sufrir cambios repentinos. En el caso de la sección divergente, el incremento de la temperatura en la pared, a un valor mayor de la temperatura de entrada, es consecuencia de la fricción del flujo a alta velocidad rozando con la pared adiabática, el cual disminuye drásticamente antes de salir el flujo de la tobera. Mientras que, en la simetría axial, la temperatura disminuye producto de la expansión del flujo, y se muestra la fluctuación de su magnitud a partir de la posición $x=22,5 \mathrm{~mm}$. $\mathrm{Y}$ de los perfiles de densidad (Figura 20) muestran su comportamiento en función de la expansión y compresión del flujo, en las secciones convergente, de la garganta y en la divergente.

Los resultados del campo de flujo y de los perfiles que definen la trayectoria de la presión, número de Mach, temperatura, velocidad y densidad, en la 
simetría axial y en la pared de la tobera, son aceptables. Esto se justifica porque la diferencia de magnitud de número de Mach es de 0,117, entre el valor teórico de Mach 3,217 obtenido con la Ecuación (7) y el valor numérico de Mach 3,1 mostrado en la Figura 17, siendo ambos valores calculados a la salida de la tobera y de magnitud cercanos entre sí.

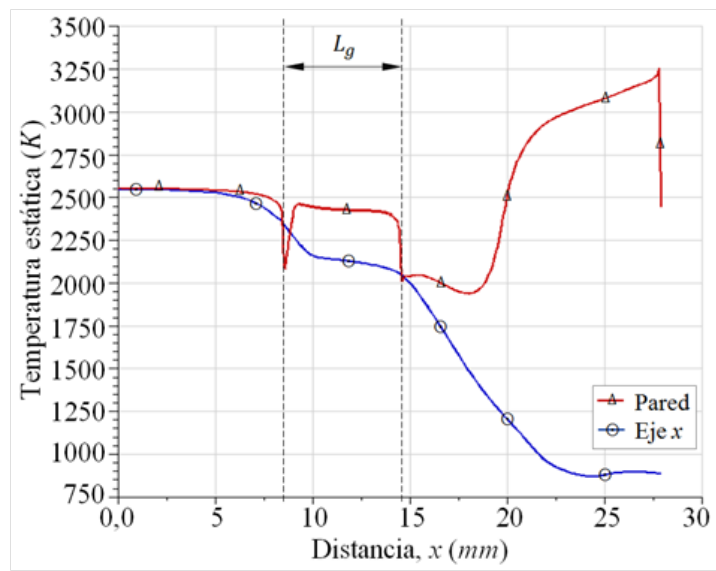

Figura 19. Perfiles de temperatura estática evaluados en la pared y en el eje X, en la sección de la tobera

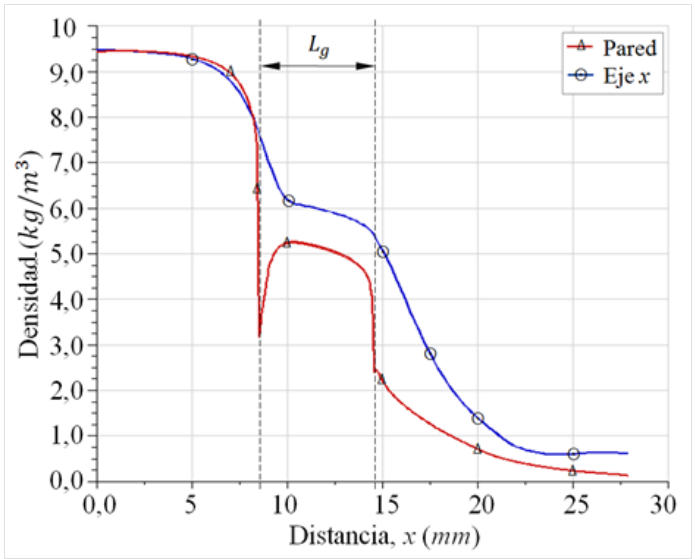

Figura 20. Perfiles de densidad evaluados en la pared y en el eje $\mathrm{X}$, en la sección de la tobera

Los detalles ampliados de la sección de la garganta muestran el comportamiento de las trayectorias de los perfiles de presión estática (Figura 21), número de Mach (Figura 22), velocidad (Figura 23), temperatura estática (Figura 24) y densidad (Figura 25), para diferentes distancias radiales, evaluadas desde la simetría axial hacia la pared. La longitud de garganta inicia en la posición $x=8,51 \mathrm{~mm}$ y termina en la posición $x=14,53 \mathrm{~mm}$. Los perfiles muestran el comportamiento de la región del flujo en el primer vértice, al inicio de la longitud de garganta, a lo largo de la longitud de garganta, así como, cuando el flujo ingresa al inicio de la sección divergente.
Para el caso de número de Mach (Figura 22) y la velocidad (Figura 23), para la región del flujo adyacente a la pared de la sección de la garganta, el flujo se acelera, desacelera y vuelve acelerar; mientras que, en la simetría axial, el flujo se acelera, desacelera y vuelve a acelerar al ingresar a la sección divergente; y esto es consecuencia de las variaciones de presión estática que se presentan desde el inicio de la sección de la garganta hasta el final de dicha sección (ver Figura 21). Por lo cual, la geometría del perfil aerodinámico de la sección de la garganta de longitud $L_{g}$, determina el comportamiento del patrón del flujo en dicha sección, en este caso, se tiene un patrón de flujo en la sección de la garganta, para un flujo subexpansionado en la salida de la tobera a velocidad supersónica mayor de Mach 3.

Las variaciones de la temperatura estática en la sección de la garganta se muestran en la Figura 24, donde en la pared se presenta un incremento de su magnitud, y variaciones en los vértices a la entrada y salida de la garganta (Figura 24). Asimismo, para el caso de la densidad (Figura 25), hacia la pared disminuye su magnitud, y en el vértice a la entrada de la garganta, la densidad disminuye e incrementa, con un comportamiento similar a la trayectoria del perfil de la presión.

Los resultados muestran que la geometría de la sección de la garganta con relación de longitud de garganta y diámetro $r_{L D}=1,07$, inicia en la posición $x=8,51 \mathrm{~mm}$ y termina en la posición $x=14,53 \mathrm{~mm}$, la cual es un tramo corto de sección circular, influye en el desarrollo del flujo en las regiones adyacentes a las paredes, en los vértices y en la simetría. En la simetría axial, en el eje X, el flujo alcanzó un valor en el rango de Mach 0,65 a 1,2, con regiones de velocidad subsónica, transónica y sónica, sin presencia de choque; por lo cual, se presenta un patrón de flujo.

En un trabajo reportado de la tobera cónica experimental ULA-1A XP [11], con ángulo medio de la sección divergente de $11^{\circ}$ y relación longitud de garganta y diámetro $r_{L D}=1,10$, presentó desaceleración del flujo con presencia de ondas de choque oblicuas, en el rango de Mach 0,8 a 1,4; para un flujo sobreexpandido.

Al comparar ambos casos, para el flujo en la sección de la garganta, la longitud de la misma influye en el desarrollo del flujo, sea un flujo subexpandido o sobreexpandido.

Otro patrón de flujo se presentó a partir de la posición $x=22,5 \mathrm{~mm}$. Desde la posición de $x=22,5 \mathrm{~mm}$ hasta la salida de la tobera, el comportamiento de la trayectoria de la curva muestra que se produce una desaceleración del flujo en la divergente, luego que el flujo alcanza la velocidad supersónica Mach 3.

Resultados similares de la desaceleración del flujo en la divergente para dominios $2 \mathrm{D}$, fue reportado en [19] mediante un patrón de densidad, para un ángulo 
medio de $11,01^{\circ}$ de una tobera plana, para condiciones de flujo sobre-expandido. Otro estudio reportó desaceleración del flujo para una tobera cónica de ángulo medio menor de $5^{\circ}$ [27], así como, para una tobera con contorno optimizado y de contorno parabólico [28].

Por lo cual, no es un resultado aislado de la fluctuación obtenido en el presente trabajo, al presentar una región del flujo una desaceleración en la sección divergente, con un patrón definido.

Cabe destacar, en la literatura para flujo unidimensional invíscido $[1,4,5,9,26]$, detallan el incremento de la velocidad del flujo de acuerdo con el número de Mach en la divergente de una tobera, la trayectoria de la curva es creciente y tiende a curvarse hacia la salida de la misma, y no presenta fluctuación alguna. Sin embargo, los resultados del presente trabajo muestran que se produce una fluctuación para cierta región del flujo en la divergente, a pesar de que la onda de choque se presenta fuera de la tobera.

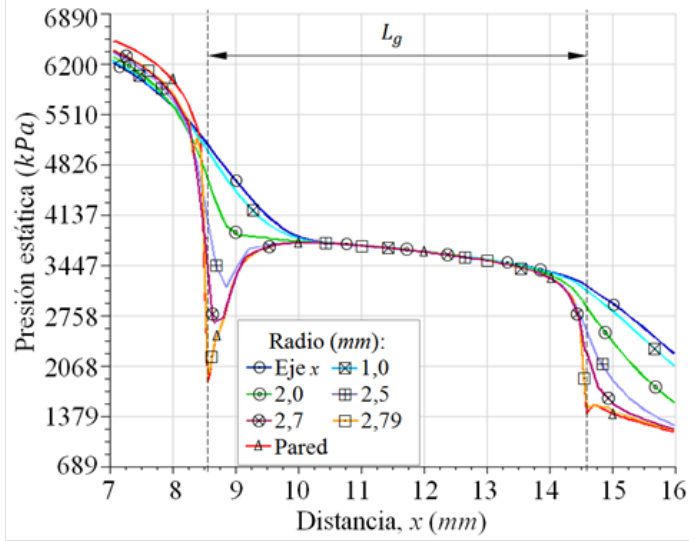

Figura 21. Perfiles de presión estática evaluados en la sección de la garganta de la tobera

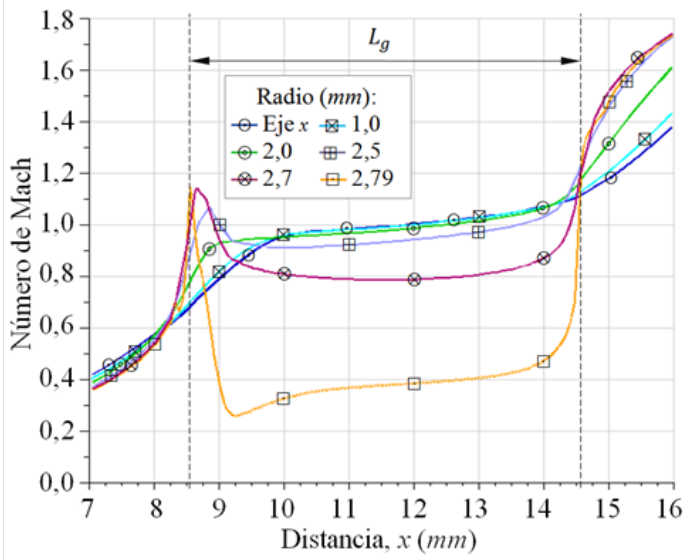

Figura 22. Perfiles de número de Mach evaluados en la sección de la garganta de la tobera

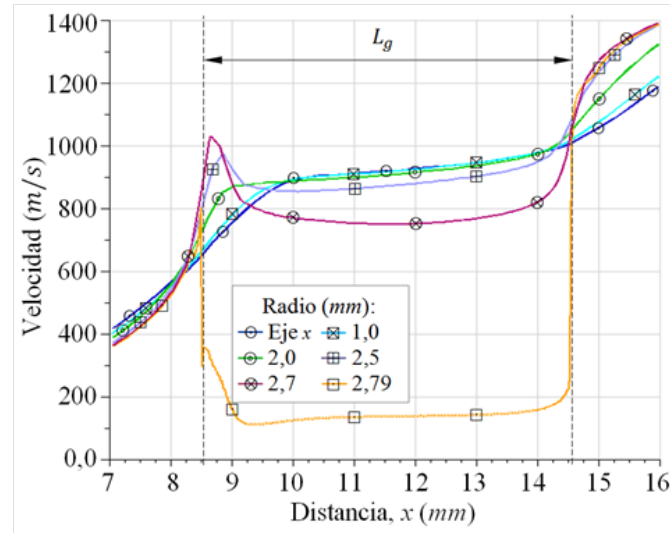

Figura 23. Perfiles de velocidad evaluados en la sección de la garganta de la tobera

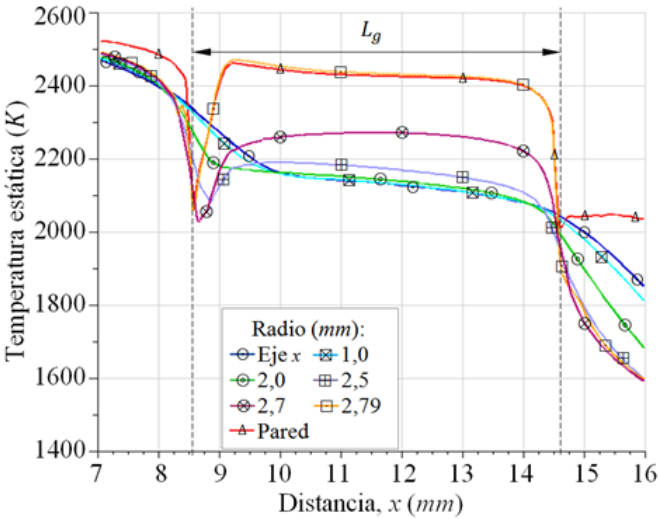

Figura 24. Perfiles de temperatura estática evaluados en la sección de la garganta de la tobera

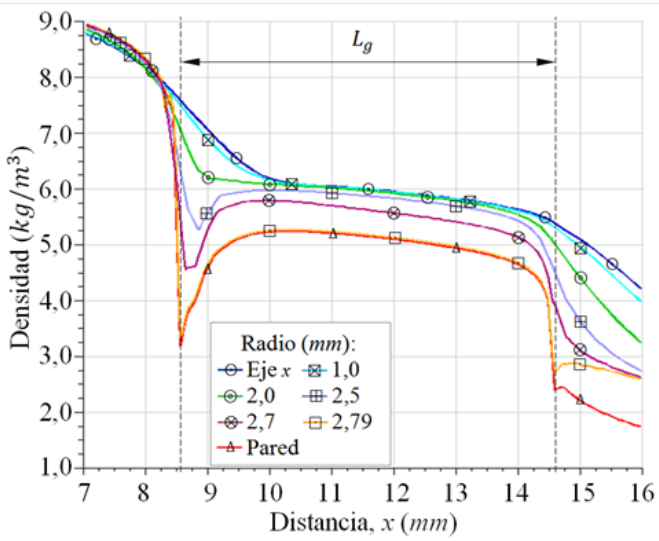

Figura 25. Perfiles de densidad evaluados en la sección de la garganta de la tobera

\section{Conclusiones}

De acuerdo con los análisis realizados, de los resultados de las simulaciones numéricas del flujo subexpansionado, se concluye que: 
En la sección de la tobera, se presentan dos regiones donde la caída de presión del flujo se lentifica, en la sección de longitud de garganta, que se encuentra entre la posición $x=8,51 \mathrm{~mm}$ y $x=14,53 \mathrm{~mm}$; y al final de la sección divergente, a partir de la posición $x=22,5 \mathrm{~mm}$ hasta la salida de la tobera. Por lo cual, en ambas regiones, el flujo se desacelera.

En la simetría axial, al final de la sección divergente de la tobera, el flujo alcanza un valor ligeramente mayor de Mach 3, y velocidad del flujo de $1800 \mathrm{~m} / \mathrm{s}$. En el ambiente de la atmósfera alcanza un valor estimado de Mach 4,75, y velocidad del flujo de $2050 \mathrm{~m} / \mathrm{s}$, antes que se produzca el choque. Aguas abajo, las magnitudes de número de Mach y de velocidad fluctúan, hasta una distancia prolongada, alrededor de Mach 3.

Los perfiles de presión estática, número de Mach, velocidad, temperatura estática y densidad, muestra cómo se desarrolla el flujo en la sección de la garganta, donde al inicio de la sección de la garganta, está presente el vértice; una región del flujo adyacente a la pared presenta fluctuaciones, ocasionando caídas de presión, por lo cual, la velocidad del flujo en esa región adyacente a la pared se acelera y desacelera.

En la simetría axial, en la garganta, en la posición $x=8,51 \mathrm{~mm}$ el flujo alcanza un valor estimado de Mach 0,68, al final de la sección de la garganta, en la posición $x=14,53 \mathrm{~mm}$ un valor estimado de Mach 1,1; por lo cual, una región del flujo es subsónica y el resto transónica. En dicha sección de la garganta, la trayectoria del perfil define un comportamiento escalonado.

Se considera un trabajo en el futuro, para dominios $2 \mathrm{D}$ y $3 \mathrm{D}, \mathrm{y}$ con diferentes códigos computacionales, reducir de manera progresiva la longitud de garganta $L_{g}$, para determinar si persiste la influencia en la aceleración del flujo o si se presenta una posible fluctuación que origine ondas de choque oblicuas. Asimismo, determinar si la fluctuación de los parámetros termodinámicos, tales como el número de Mach, presión y temperatura, en la simetría axial, al final de la sección divergente presenta algún cambio significativo.

\section{Agradecimientos}

Mi agradecimiento a Jehová, mi Dios todopoderoso, mi fuente de sabiduría e inspiración. Al Centro de Estudios Energéticos de la Universidad Nacional Experimental Politécnica «AJS» Vice-Rectorado Puerto Ordaz (UNEXPO), Bolívar, Venezuela. Al Grupo de Modelamiento Matemático y Simulación Numérica (GMMNS, Group of Mathematical Modeling and Numerical Simulation) de la Universidad Nacional de Ingeniería (UNI), Lima, Perú. A Richard Nakka (Ingeniero Aeroespacial), por el aporte de datos experimentales de la tobera del motor cohete Helios-X.

\section{Referencias}

[1] G. P. Sutton and O. Biblarz, Rocket propulsion elements. John Wiley \& Sons, 2016. [Online]. Available: https://bit.ly/35psFaR

[2] J. Blazek, Computational fluid dynamics: principles and applications. Butterworth-Heinemann, 2015. [Online]. Available: https://bit.ly/3pnxjhx

[3] B. Andersson, R. Andersson, L. Hakansson, M. Mortensen, R. Sudiyo, and B. van Wachem, Computational Fluid Dynamics Engineers. Cambridge University Press, 2011. [Online]. Available: https://bit.ly/32BPCWo

[4] J. D. Anderson, Fundamentals of aerodynamics. McGraw-Hill international editions. Mechanical engineering series, 1984. [Online]. Available: https://bit.ly/3eQR5ge

[5] F. M. White, Fluid Mechanics. McGraw-Hill series in mechanical engineering, 2011. [Online]. Available: https://bit.ly/35opmAy

[6] P. Krehl and S. Engemann, "August toepler the first who visualized shock waves," Shock Waves, vol. 5, no. 1, pp. 1-18, Jun. 1995. [Online]. Available: https://doi.org/10.1007/BF02425031

[7] V. Karman, "The fundamentals of the statistical theory of turbulence," Journal of the Aeronautical Sciences, vol. 4, no. 4, pp. 131-138, 1937. [Online]. Available: https://doi.org/10.2514/8.350

[8] F. White, Viscous fluid flow. McGraw-Hill series in Aeronautical and Aerospace Engineering, 1974. [Online]. Available: https://bit.ly/3eRRCyP

[9] H. Schlichting and K. Gersten, Boundary-Layer Theory. Springer, 2016. [Online]. Available: https://bit.ly/36yZGAx

[10] D. C. Wilcox, Turbulence Modeling for CFD. DCW Industries, Incorporated, 1994. [Online]. Available: https://bit.ly/32HZnCm

[11] A. L. Tolentino, J. Ferreira, M. Parco, L. Lacruz, and V. Marcano, "Simulación numérica del flujo sobre-expandido en la tobera cónica experimental ULA-1A XP," Unviversidad, Ciencia y Tecnología, vol. 21, no. 84, pp. 126-133, 2017. [Online]. Available: https://bit.ly/2H4yX6k

[12] V. Marcano, P. Benitez, C. La Rosa, L. La Cruz, M. A. Parco, J. Ferreira, R. Andrenssen, A. Serra Valls, M. Peñaloza, L. Rodríguez, J. E. Cárdenas, V. Minitti, and J. J. Rojas, "Progresos alcanzados en el proyecto universitario cohete sonda ULA," Universidad, Ciencia y Tecnología, vol. 13, no. 53, pp. 305-316, 2009. [Online]. Available: https://bit.ly/3f73vB2 
[13] L. Lacruz-Rincón, M. A. Parco-Brizuela, R. Santos-Luque, C. Torres-Monzón, J. FerreiraRodríguez, and P. Benítez-Díaz, "Análisis experimental de las oscilaciones de presión interna en un motor de combustible solido para cohete sonda," Ciencia e Ingeniería, vol. 13, no. 53, 2016. [Online]. Available: https://bit.ly/3noQfdL

[14] Universidad de los Andes. Programa espacial ULA. [Online]. Available: https://bit.ly/35tiodw

[15] S. L. Tolentino Masgo and R. Nakka, "Simulación del flujo supersónico en la tobera del motor cohete Helios-X de categoría amateur," in Jornadas de Investigación, 2019. [Online]. Available: https://bit.ly/3pwDY8U

[16] R. Nakka. Richard Nakka's experimental rocketry web site. [Online]. Available: https://bit.ly/2IrDZKX

[17] F. R. Menter, "Two equation eddy-viscosity turbulence models for engineering applications," Aerospace Research Central, vol. 32, no. 8, pp. 1598-1605, 2012. [Online]. Available: https://doi.org/10.2514/3.12149

[18] A. Balabel, A. M. Hegab, M. Nasr, and S. M. ElBehery, "Assessment of turbulence modeling for gas flow in two-dimensional convergent-divergent rocket nozzle," Applied Mathematical Modelling, vol. 35, no. 7, pp. 3408-3422, 2011. [Online]. Available: https://doi.org/10.1016/j.apm.2011.01.013

[19] S. L. Tolentino Masgo, "Evaluación de modelos de turbulencia para el flujo de aire en una tobera plana," INGENIUS, no. 22, pp. 25-37, 2019. [Online]. Available: https://doi.org/10.17163/ings.n22.2019.03

[20] Y. Liu, J. Wu, and L. Lu, "Performance of turbulence models for transonic flows in a diffuser," Modern Physics Letters B, vol. 30, no. 25, p. 1650326, 2016. [Online]. Available: https://doi.org/10.1142/S0217984916503267

[21] S. L. B. Tolentino Masgo, "Evaluación de modelos de turbulencia para el flujo de aire en un difusor transónico," Revista Politécnica, vol. 45, no. 1, pp. 25-38, abr. 2020. [Online]. Available: https://doi.org/10.33333/rp.vol45n1.03

[22] Y. Zhang, H. Chen, M. Zhang, M. Zhang, Z. Li, and S. Fu, "Performance prediction of conical nozzle using navier-stokes computation," Journal of Propulsion and Power, vol. 31, no. 1, pp. 192-203, 2015. [Online]. Available: https://doi.org/10.2514/1.B35164

[23] R. Jia, Z. Jiang, and W. Zhang, "Numerical analysis of flow separation and side loads of a conical nozzle during staging," Proceedings of the Institution of Mechanical Engineers, Part G: Journal of Aerospace Engineering, vol. 230, no. 5, pp. 845-855, 2016. [Online]. Available: https://doi.org/10.1177/0954410015599798

[24] H. Ding, C. Wang, and G. Wang, "Transient conjugate heat transfer in critical flow nozzles," International Journal of Heat and Mass Transfer, vol. 104, pp. 930-942, 2017. [Online]. Available: https://doi.org/10.1016/j. ijheatmasstransfer.2016.09.021

[25] A. K. Mubarak and P. S. Tide, "Design of a double parabolic supersonic nozzle and performance evaluation by experimental and numerical methods," Aircraft Engineering and Aerospace Technology, vol. 91, no. 1, pp. 145-156, Dec. 2020. [Online]. Available: https://doi.org/10.1108/AEAT-12-2017-0275

[26] R. H. Pletcher, J. C. Tannehill, and D. Anderson, Computational Fluid Mechanics and Heat Transfer. CRC Press, 2012. [Online]. Available: https://bit.ly/3psYKX9

[27] D. Munday, E. Gutmark, J. Liu, and K. Kailasanath, Flow Structure of Supersonic Jets from Conical C-D Nozzles. [Online]. Available: https://doi.org/10.2514/6.2009-4005

[28] J. Östlund and B. Muhammad-Klingmann, "Supersonic Flow Separation with Application to Rocket Engine Nozzles ," Applied Mechanics Reviews, vol. 58, no. 3, pp. 143-177, 05 2005. [Online]. Available: https://doi.org/10.1115/1.1894402 


\title{
REDUCIENDO LA BRECHA DE SEGURIDAD DEL IOT CON UNA ARQUITECTURA DE MICROSERVICIOS BASADA EN TLS Y OAUTH2
}

\section{REDUCING THE IOT SECURITY BREACH WITH A MICROSERVICE ARCHITECTURE BASED ON TLS AND OAUTH2}

\author{
Diego Ordóñez-Camacho ${ }^{1, *}$
}

Recibido: 15-09-2020, Revisado: 19-10-2020 Aprobado tras revisión: 14-11-2020

\section{Resumen}

El Internet de las cosas es una de las tendencias más prometedoras en la actualidad. La rapidez de su adopción, sin embargo, ha provocado ciertas brechas críticas en la seguridad de los sistemas involucrados. Este proyecto analizó el problema de seguridad de una manera amplia, pero enfocándose en entornos de tipo hogar inteligente, donde el uso de dispositivos con tecnologías ampliamente heterogéneas genera problemas en la autenticación con múltiples servicios, y en la confidencialidad de los datos, si la red llegara a verse comprometida. Para atacar estos problemas, se juntaron tecnologías de última generación como OAuth2 y TLS, entre otras, junto a una metodología arquitectural de microservicios de acoplamiento ligero, para generar una arquitectura IoT segura y de amplio alcance, respaldada y validada por una implementación de referencia. La división en capas funcionales permite que tanto los dispositivos y sensores fijos como aquellos móviles, puedan acoplarse al sistema de manera transparente y fluida. El esquema de seguridad estructurado en tres niveles incrementales permite que cada equipo pueda integrarse al que mejor se adapte tanto a sus recursos computacionales como al tipo de información que debe entregar o consumir. Los resultados muestran la flexibilidad de la solución y la solidez del esquema de seguridad presentado.

Palabras clave: IoT, microservicios, arquitectura de software, seguridad de sistemas, TLS, OAuth

\section{Abstract}

The Internet of Things has emerged as one of the most promising trends today. The speed of its adoption, however, has caused certain gaps. Amongst the most critical there is the one related with the security of the systems involved. This project addressed the security problem in a broad way but focusing on smart-home environments, where the use of devices with widely heterogeneous technologies and multiple services, generates problems with authentication and with the confidentiality of the data, if the network is compromised. To tackle these problems, state-of-theart technologies such as OAuth2 and TLS, among others, were put together, along with an architectural methodology of lightly coupled microservices. As a result, a secure and broad range IoT architecture was built, backed up and validated by a reference implementation. The division into functional layers enables both fixed and mobile devices and sensors, to get connected into the system transparently and fluently. The security scheme structured in three incremental levels enables a better device integration, at the level that best adapts to its computing resources and the type of information it shares. The results show the flexibility of the solution and the robustness and novelty of the security scheme presented.

Keywords: IoT, microservices, software architecture, systems security, TLS, OAuth.

\footnotetext{
${ }_{1, *}$ Grupo de Investigación en Informática (GrIInf), Universidad UTE - Ecuador.

Autor para correspondencia dordonez@ute.edu.ec. (D) http://orcid.org/0000-0001-8390-634X
}

Forma sugerida de citación: Ordóñez-Camacho, D. (2021). «Reduciendo la brecha de seguridad del IoT con una arquitectura de microservicios basada en TLS y OAuth2». InGEnIUS. N. ${ }^{\circ}$ 25, (enero-junio). pp. 94-103. DOI: https://doi.org/10.17163/ings.n25.2021.09. 


\section{Introducción}

El Internet de las cosas, IoT por sus siglas en inglés (Internet of Things), es una tecnología que ingresa con fuerza en la realidad de las personas. Todos los entornos están involucrados, urbanos, industriales, de oficina o el hogar. El interés generado y la rapidez de adopción de la tecnología han producido un cierto desorden e informalidad en el proceso. Esto tiene como consecuencia que elementos importantes se dejaron de lado, uno de los más relevantes es el de la seguridad [1].

En principio, la seguridad del IoT no tiene por qué distar de la seguridad en una red típica de computadores. En la práctica, sin embargo, hay dificultades propias del entorno que complican aún más el problema de la seguridad. Muchos dispositivos para IoT son limitados computacionalmente, lo que impide utilizar varios mecanismos robustos de seguridad conocidos. La gran cantidad de dispositivos que pueden estar involucrados en una red IoT, así como el incremento exponencial en el número de interacciones, agrava el problema. La diversidad de los equipos que se utilizan, tanto en hardware como en software, complican la posibilidad de generalizar las soluciones propuestas [2]. Hay una gran variedad de métodos y herramientas que pueden utilizarse para acometer la labor [3]. En este trabajo la intención fue la de utilizar aquellas técnicas que, así como el IoT, marcan tendencia y son utilizadas exitosamente en otros campos relacionados. De entre estas técnicas, las más prometedoras fueron los microservicios [4] y OAuth2 [5], las cuales, al aunarse con técnicas y tecnologías, tal vez un poco más tradicionales a la base, pero igualmente exitosas como TLS [6] y MQTT [7], proporcionaron un entorno estructurado apropiado.

El objetivo general fue proporcionar al mundo del IoT una alternativa arquitectural segura y adaptada a las nuevas tendencias tecnológicas, que pueda ser utilizada de manera genérica en una multitud de situaciones. Más específicamente, primero, se dio especial relevancia a generar una alternativa para hogares inteligentes, por esto la metodología propicia la clara división de funciones, pero cuidando la integración fluida y la disponibilidad de herramientas para la creación de nuevas utilidades. Segundo, de entre los múltiples problemas de seguridad existentes, se atacó a aquellos relacionados con la autenticación de clientes al llamar a múltiples servicios y con la confidencialidad al transmitir información, especialmente al comprometerse la red. Finalmente, conscientes de las restricciones de hardware de muchos dispositivos, sobre todo, sensores, se trabajó en una arquitectura que contemple una jerarquía de varios niveles de seguridad, permitiendo una interconexión ajustada a las capacidades de varios tipos de equipos, especialmente en ambientes con tecnologías heterogéneas.

\subsection{Trabajos relacionados}

En el ecosistema de dispositivos IoT, estos son en gran medida poco seguros, por ser equipos pequeños y de bajo consumo energético, por ende, también tienen recursos computacionales limitados. Esta última condición afecta mucho al momento de intentar incluir en ellos esquemas de seguridad complejos [8]. A nivel industrial hay varios emprendimientos aplicando IoT, por ejemplo, en el transporte inteligente $[9,10]$ y en la agricultura [11]; sin embargo, uno de los grandes objetivos actuales relacionados con la automatización en el hogar y oficina es la vida conectada (connected living). Este objetivo requiere de importantes avances en el campo del IoT, donde se requiere brindar respuestas para los problemas relacionados con el enorme incremento de dispositivos que deberán interactuar [12]. Un caso particular en el IoT es aquel de los hogares inteligentes, dado que muchas veces las soluciones que se implementan son $a d$-hoc, por parte de los mismos usuarios, quienes suelen intentar reducir al máximo costos y esfuerzos, lo cual generalmente se ve reflejado en un esquema de seguridad mínimo y probablemente inexistente [13].

Los dispositivos involucrados requieren interconectarse en un esquema de muchos-a-muchos. Para asegurar el intercambio de información es necesario implementar un sistema de administración de identidades que escale apropiadamente. En este sentido, Ayed, Boujezza y Riabi [14] proponen un sistema para el hogar que combina EAP, OAuth y DTLS. También preocupados por la administración de identidades y el control de acceso, Fernández, Alonso, Marco y Salvachúa [15] confirman las posibilidades de OAuth y hacen una propuesta arquitectural compatible con servicios.

Bugeja, Jacobsson y Davidsson [16] analizan la problemática de la seguridad IoT en el hogar y destacan lo importante que es evitar que los sensores capturen y distribuyan, de manera indiscriminada, los datos del hogar. A manera de ejemplo presentan el caso de las conversaciones privadas, las cuales no deberían ser publicadas. Entre los enfoques posibles, mencionan como una alternativa viable, aquel orientado a servicios, para equilibrar entre centralización y distribución del control; más aún, la tendencia en software y aplicaciones distribuidas parecería dirigirse en general hacia el empleo de microservicios [17-19]. Ahondando en esta línea, Díaz-Sánchez, Marín-López, Almenarez, Arias y Sherrat [20] destacan los beneficios que una arquitectura de microservicios, adicionalmente basada en TLS/PKI, puede tener en IoT, al aligerar las tareas de desarrollo y mantenimiento, beneficioso tanto para proveedores y distribuidores como para usuarios, al mismo tiempo, reforzando la seguridad de interconexión. Estudios de caso como el de Urien [21] confirman de manera práctica las posibilidades de estas técnicas. 
Si bien en la mayoría de los trabajos relacionados es SSL/TLS el mecanismo de seguridad y cifrado preferido, es muy interesante lo que proponen $\mathrm{Hoz}$ et al., [22] al analizar las complicaciones que a nivel práctico pueden existir en IoT con TLS. Dicho trabajo, entonces, analiza las posibilidades de utilizar SSH y destaca las ventajas que proporciona el tema de compresión de datos incluido en dicho protocolo, que especialmente se muestra ventajoso al trabajar sobre HTTP.

Khan, Anwar, Azam, Samea y Shinwari [23] aportan al entorno del IoT con un enfoque administrado por modelos y proponen justamente un modelo orientado a OAuth con una fuerte inclinación UML. Esta propuesta, a través de transformaciones, podría ser adaptada a una arquitectura específica, brindando la posibilidad de personalizarse al entorno requerido. Otra interesante propuesta arquitectural y de seguridad es aquella presentada por Kim, Wasicek, Mehne y Lee [24], donde en lugar de un mecanismo tradicional como el presentado por las autoridades de certificación en SSL, se utilizaría un acercamiento de autoridades de certificación locales, las cuales de manera más frecuente, pero también con un proceso más ligero, autenticarían los equipos de IoT. Se puede considerar que un punto medio entre las dos propuestas que acabamos de revisar sería aquella de Pahl y Donini [25] la cual usa certificados tradicionales, pero con autenticación próxima, más bien a nivel de nodos; destacan que este mecanismo podría complementarse con alguno de autorización, como OAuth o similares.

Sciancalepore, Piro, Caldarola, Boggia y Bianchi [26] dan énfasis a OAuth, pero sobre todo, con la particularidad de concentrar su arquitectura de seguridad en el equipo gateway, donde reside la estación de base o sink node, que se encarga del procesamiento pesado de autenticar, autorizar y establecer los lazos entre clientes y recursos. Este enfoque es muy relevante cuando consideramos lo susceptibles que son aquellos equipos de borde ligados al edge computing [27], a través de los cuales se puede comprometer todo un sistema de IoT. Uno de los puntos álgidos de la seguridad en los sistemas de borde en IoT suele ser el relacionado con el uso de MQTT (o protocolos similares), por lo cual varios trabajos, como el de Singh, Rajan, Shivraj y Balamuralidhar [28] proponen mejoras sobre dicho protocolo.

\section{Materiales y métodos}

Para este trabajo se consideró principalmente que dentro del ecosistema IoT es necesario segmentar la ubicación, alcance y acceso de los equipos involucrados en dos capas: local o de borde y centralizada.

En la capa local, representada esquemáticamente en la Figura 1, tenemos aquellos elementos que es- tarán invariablemente instalados en la casa u oficina inteligente, como es el caso de sensores, actuadores, procesadores de borde como gateways y brókeres, y dispositivos móviles de usuario.

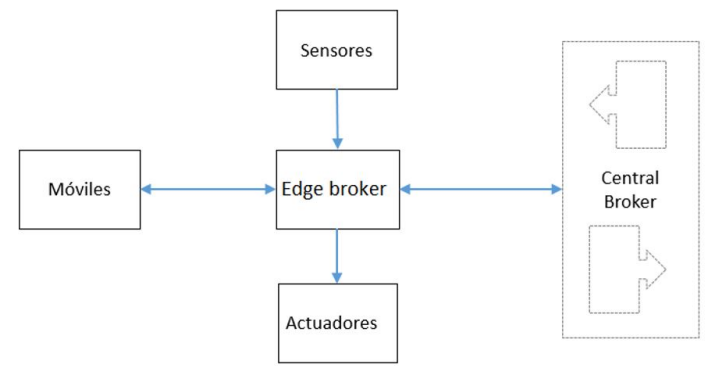

Figura 1. Componentes en la capa local o de borde del sistema IoT

Los sensores son todo equipo capaz de capturar fenómenos físicos, eventos virtuales o señales periódicas. Aquellos sensores con la capacidad necesaria pueden comunicarse directamente con el bróker central, caso contrario interactuarán con equipos en el subsistema de procesamiento de borde. Los sensores serán estáticos, cuando emitan una señal constante que sería utilizada generalmente por equipos móviles desplazándose en el entorno como balizas Bluetooth para posicionamiento. Los sensores dinámicos capturarán mediciones del ambiente, las cuales variarán dependiendo de las condiciones del entorno, como luminiscencia, temperatura, humedad, entre otros.

Los actuadores permitirán la interacción con hardware o software generando eventos o acciones. Recibirán instrucciones sea directamente del bróker, o desde algún preprocesador cuando es necesario. Se dividen en locales, ubicados en el entorno inteligente, como controladores de luz o temperatura; pueden ser también remotos como aquellos capaces de enviar instrucciones, probablemente por la red, para algún equipo distante, pero controlado desde la casa u oficina inteligente, como cuando se requiere enviar un sms, correo o tuit.

Finalmente, esta capa contempla los equipos preprocesadores, que también pueden llamarse procesadores o brókeres de borde. Estos capturan información en bruto proveniente de los sensores para reenviarla al bróker centralizado o al actuador cuando el sensor es incapaz. La información se podrá enviar tal como se recibió del sensor, o preprocesarla y enviar este resultado. Estos equipos pueden ser Raspberri Pi o computadoras pequeñas como las tabletas.

En la capa centralizada, presentada en la Figura 2, se consideran los equipos encargados de la coordinación general de todos los componentes y se considera que son necesarios tres subsistemas: administración, procesamiento y persistencia. Estos subsistemas se ligan entre ellos y también con la capa de borde mediante un bróker centralizado. 


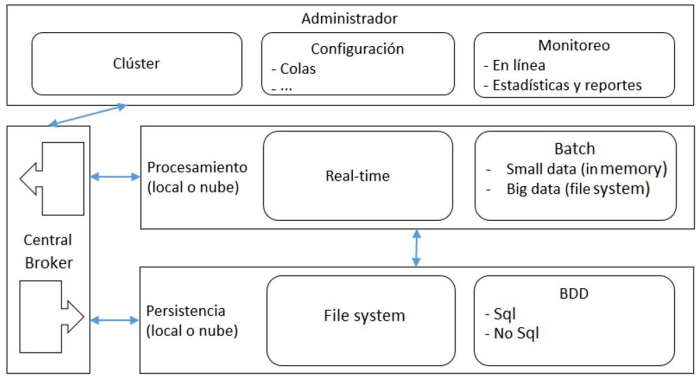

Figura 2. Componentes en la capa centralizada del sistema IoT

El subsistema de administración define los parámetros y la configuración del sistema presenta las interfaces web para que los usuarios administradores interactúen con todo el sistema. En el caso de que el procesamiento central se haga con varios equipos, también administra el clúster resultante. Parte principal dentro de este subsistema es entonces el monitoreo, que permitirá a todo tipo de usuarios revisar la información pertinente, de preferencia mediante dashboards y cuadros estadísticos.

Para todo el procesamiento pesado de información, el subsistema correspondiente toma los datos recolectados desde el bróker y los procesa según se defina por las aplicaciones o necesidades específicas del sistema IoT. En general el procesamiento se dividirá dependiendo, sobre todo, de la urgencia del procesamiento, en real-time, que procesa los datos en un flujo continuo, según vayan llegando de los sensores; in memory, que recolecta la información en la memoria del clúster, dependiendo de las necesidades, y la procesa en pequeños lotes; y batch, que interactúa en general con el sistema de almacenamiento, para procesamientos donde la cantidad de datos es mayor que la que cabe en la memoria viva del sistema.

La información generada por el sistema IoT se direcciona al módulo de persistencia, el cual salvaguarda los datos para su uso posterior sea en el desarrollo de modelos o en la generación de reportes. Varias alternativas deben considerarse, dependiendo del tamaño de la información y la forma como será accedida. Al menos es necesario considerar un soporte HDFS, uno de bases de datos tanto SQL como NoSQL, y en caso de que la evolución del sistema así lo requiera, un soporte en la nube.

Finalmente, todo el sistema, y más específicamente las capas de borde y centralizada, deben conectarse e intercambiar información, lo cual se consigue mediante un bróker central, encargado de manejar todas las colas de mensajes reduciendo así la complejidad de las interacciones.

\section{Resultados}

El diseño arquitectural resultante tomó en consideración, sobre todo, la necesidad de asegurar el intercambio de información de todos los componentes del sistema, procurando cuidar en todo momento la rapidez de cálculo. Estos elementos requieren conciliar características que muchas veces son incompatibles. Por ejemplo, sistemas de criptografía más robustos pueden requerir más poder de cómputo del que muchos dispositivos ligeros, como sensores, proporcionan.

La arquitectura final diseñada, implementada y probada, es aquella esquematizada en la Figura 3, que se describirá en detalle a continuación. En primer lugar, se puntualizarán los componentes involucrados, para luego presentar la funcionalidad de seguridad en general.

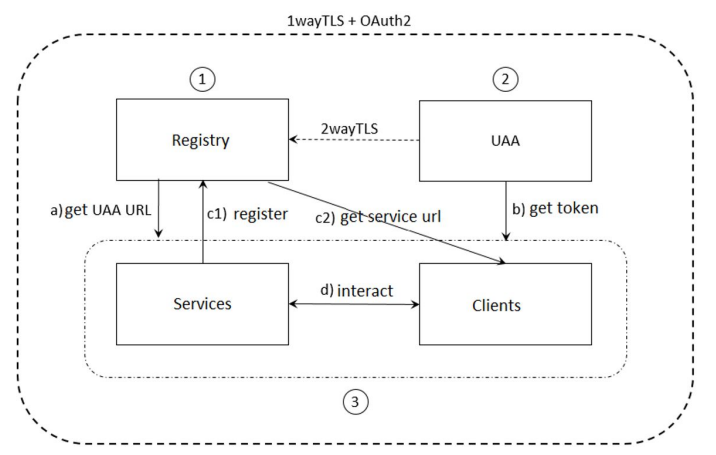

Figura 3. Arquitectura segura

\subsection{Componentes}

En esta sección se intentarán definir de manera general los tipos de componentes o equipos involucrados en la arquitectura propuesta en la Figura 3, en la cual se cuenta básicamente con los encargados del servicio de registro (Registry), los equipos brindando servicios de autenticación para clientes y usuarios (UAA) y luego ya, de forma amplia, todos los equipos brindando servicios generales, así como todos los equipos cliente (Services y Clients). Si bien por simplicidad se hará referencia a los componentes en singular, la arquitectura considera que, para cada categoría o tipo de componente, especialmente los servicios, estos pueden trabajar en clústeres.

\subsubsection{Servicios de registro (REG)}

La labor principal del REG es permitir a los servicios registrarse mediante IP y alias (nombre de servicio) y ponerlos así a disposición de los clientes, quienes se conectarán al REG para pedir la información con la cual se conectarán finalmente a los servicios de interés. El REG proporciona también un servicio de balanceo de carga, al detectar que un servicio está registrado en clúster (varios equipos con el mismo servicio). 
El primer punto de contacto para todos los demás componentes del sistema, sean estos servicios o clientes, es el REG, el cual requiere una IP estática; todos los demás componentes del sistema, sin embargo, pueden trabajar con IP dinámicas, mediante un DNS.

\subsubsection{Servicios de autenticación (UAA)}

El UAA toma sus siglas del inglés User Authentication and Authorization. Dentro de nuestra arquitectura básicamente nos proporciona el servicio de autenticación, que trabaja bajo OAuth2. El UAA guarda los datos de todos los clientes en el sistema, incluyendo sus roles; con esta información los servicios usuarios del UAA pueden también autorizar o no el uso de ciertos elementos. Cualquier componente puede conectarse con el UAA para, mediante sus credenciales de cliente (usuario y password) solicitar un token de acceso. De la misma manera, cualquier componente del sistema puede solicitar al UAA la validación de un token recibido por parte de un tercero.

\subsubsection{Servicios genéricos (SRV) y equipos cliente (CLI)}

La última categoría de componentes acoge todos los demás servicios y todos los clientes. En general estos componentes interactuarán entre ellos luego de haberse registrado/autenticado en el sistema con la ayuda del REG y del UAA. Los servicios pueden ser muy diversos y está en manos del administrador del sistema decidir cuáles requerirá. Sin embargo, en nuestra arquitectura para IoT, hay algunos que resultan fundamentales, por lo cual han sido implementados en el sistema de prueba, y serán mencionados a continuación.

Para permitir la interconectividad y, al mismo tiempo, reducir su complejidad, se implementó el servicio de mensajería, que en la metodología está representado por el bróker central (Figuras 1 y 2). El bróker está en capacidad de recibir y distribuir todos los mensajes circulando en el sistema y básicamente permite que en general todos los servicios y los clientes puedan establecer una conexión única con el bróker, para depositar mensajes y recuperarlos de una o más colas. Este bróker puede trabajar con cualquier protocolo de comunicación, o una combinación de ellos. Sin embargo, dado que en el mundo del IoT, al menos en la actualidad, el protocolo más expandido es el Message Queuing Telemetry Transport (MQTT), es el que se usa en la implementación que aquí se presenta.

Otro de los servicios implementados para la prueba de concepto de la arquitectura es el que tiene relación con la persistencia, como soporte necesario para posteriormente implementar procesamiento en batch. Para esto se implementó un servicio de tránsito que toma la información del bróker y la traslada a un clúster Hadoop [29], donde se pueden utilizar distintos tipos de herramientas de dicho ecosistema para el tratamiento de la información. Uno de los casos que se trabajó, dada la naturaleza de la información IoT, especialmente aquella en proveniencia de los sensores, fue el de series temporales. Para ello se construyeron dos servicios de series de datos, proporcionando de esta manera, graficación y análisis de tendencias, entre otros.

En cuanto a los clientes, aquí se consideran todos los sensores, los cuales entregan información al sistema, los actuadores, que reaccionan con el entorno gracias a la información del sistema, y todos aquellos dispositivos, móviles o de escritorio, que permitan al usuario ingresar para configurar el sistema, recolectar información procesada, o incluso actuar también como sensores y actuadores. Un móvil, por ejemplo, puede entregar información de la actividad del usuario o enviar tuits automáticamente.

\subsection{Esquemas de seguridad}

La arquitectura de seguridad del sistema comprende tres escenarios fundamentales: el básico, al cual todos los elementos deberán ceñirse en sus transacciones, salvo que se especifique de otra manera; el esquema ligero, generalmente usado únicamente al iniciar un proceso de trabajo en el sistema; y el fortalecido, para relaciones de confianza entre servicios.

\subsubsection{Esquema básico}

Este es el esquema por defecto que los componentes del sistema utilizarán en sus transacciones. Este esquema está representado en la Figura 3 por la línea punteada que engloba el sistema, y utiliza una combinación de TLS de una vía más OAuth2. Todo servicio debe proporcionar su certificado público de seguridad (PKI) a los clientes, quienes podrán así validarlo con la autoridad de certificación (CA). Asimismo, todo cliente deberá proporcionar a los servicios un token de acceso OAuth para que estos puedan validarlo con el servicio de autenticación.

El uso de TLS, en nuestro esquema, es necesario especialmente para poder cifrar el contenido de la información que se transmite. Se utiliza únicamente del lado de los servicios para limitar al máximo la sobrecarga que implicaría, sobre todo, a nivel de administración (pero también de recursos y procesamiento), utilizarlo en todos los componentes. La brecha de seguridad que aparece se compensa con el uso de OAuth2, mediante el cual los clientes son a su vez validados por los servicios.

\subsubsection{Esquema ligero}

Este es un esquema que se pudiera considerar inseguro, razón por la cual se proporciona solamente para aquellos casos donde no se puede aún obtener un token de acceso, o cuando se considera redundante solicitarlo. 
Dos casos existen al momento en el entorno de trabajo, que implementan este esquema. Cuando los componentes ingresan al sistema para poder iniciar sus transacciones, es en general necesario contar con el token OAuth, pero dado que la IP del servidor UAA puede haber cambiado, el primer paso es contactarse con REG para solicitar la IP actualizada. Para esta conexión el cliente aún no tiene el token, razón por la cual no se puede trabajar con el esquema básico. La segunda implementación de este esquema se aplicó para evitar conexiones redundantes innecesarias y se da cuando el servicio recibe el token y debe validarlo con el UAA. Dado que el servicio ya se validó inicialmente con el UAA se evita duplicar este paso.

Es para este tipo de casos que entra en juego el esquema ligero. El servicio proporciona su PKI con lo cual se cifra la comunicación, pero el cliente no está obligado a validarlo (aunque se recomienda que lo haga), el servicio proporciona un punto de acceso «inseguro» para el cliente, el cual no exige el token. Este es el esquema proporcionado por REG exclusivamente para poder entregar los datos del UAA.

\subsubsection{Esquema fortalecido}

Similar al problema trabajado en el esquema ligero, en ocasiones dos servicios requieren interconectarse, pero al menos uno de ellos (quien actúa como cliente) está en incapacidad de obtener su token de acceso. Al tratarse de servicios, no es conveniente abrir un canal inseguro como se hace en el esquema ligero. Para mantener el estándar de seguridad, entonces, se decidió implementar un esquema TLS de doble vía, lo cual es posible, sin incurrir en mayor sobrecarga, dado que, al ser servicios, ya poseen de todas maneras su PKI. Adicionalmente que en general los servicios se ejecutarán en equipos con mayor capacidad de proceso.

Esta implementación requiere también un canal dedicado para poder ejecutar este tipo de validación y el ejemplo está dado por la comunicación entre el REG y el UAA. El UAA es el que proporciona los tokens de acceso y por consiguiente debería validarse a sí mismo lo cual generaría un hueco de seguridad. El REG, entonces, abre un canal dedicado para que en todo momento un servicio UAA pueda registrarse por esa vía. Al conectarse el UAA con el REG, intercambian sus respectivos PKI, validándose mutuamente por TLS, sin reducir el estándar de seguridad del sistema.

\subsection{Funcionalidad}

Retomando la Figura 3, la línea punteada representa el alcance del esquema de seguridad básico, el cual engloba todo el sistema. Internamente pueden verse los números 1, 2 y 3 , encerrados en círculos, los que indican el orden de arranque recomendado para garantizar la fluidez del servicio. En la práctica, al menos los servicios, cuentan en su biblioteca de base con la funcionalidad para reintentar la conexión cuando este orden de inicio no se respeta. Sin embargo, esto puede ser susceptible a demoras innecesarias.

En primer lugar, se inicia el servidor de registro, REG, el cual brindará un punto central de acceso para la adquisición de la información de contacto para los demás servicios: todo servicio registrará en el REG su respectiva IP y su alias (nombre de servicio) y todo cliente buscará aquí, por alias, la IP del servicio requerido para poderse conectar con él. REG ofrece tres puntos de acceso, cada uno de los cuales debe manejar un modo de seguridad diferente: el primero, ligero, permite a cualquier cliente obtener la IP del UAA sin ninguna seguridad adicional; el segundo modo, fortalecido, permite la conexión del UAA mediante TLS de dos vías; el último, básico, que requiere de OAuth2, permite a los clientes pedir información de servicios, y a los servicios registrar su información de contacto.

En segundo lugar, se inicia un servidor de autenticación (UAA), el cual brindará credenciales OAuth2 a los clientes. Entre el UAA y el REG se utiliza el mecanismo de seguridad reforzado, con validación TLS de dos vías. El UAA se conecta con el REG, así como cualquier otro servicio, para entregarle su IP y alias y estar así disponible para todo el sistema. Una vez que estos dos servicios, REG y UAA, están en línea, todo el resto de los componentes, servicios y clientes pueden iniciar su trabajo.

Finalmente, entonces, como punto 3, cualquier otro componente, sea este servicio o cliente, procederán de la siguiente manera: en primer lugar, utilizando el esquema de seguridad ligero, se conectarán con el REG para pedir la IP del UAA; establecen la conexión con el UAA y solicitan el token de acceso, utilizando sus credenciales cliente. Con el token de acceso en mano ya se puede utilizar el esquema de seguridad básico y, en el caso de los servicios, se registrarán con el REG, entregando IP y alias, para quedar a la espera de peticiones de clientes, o actuar como cliente de otro servicio, según sea necesario. En el caso de un cliente, el siguiente paso es utilizar un servicio, donde aplicará el esquema de seguridad básico; se conecta al REG y mediante un alias solicita la IP del servicio de su interés, para luego conectarse con dicho servicio. La última acción viene a ser la de los servicios que reciben una petición de un cliente ya que en este caso deberán, mediante el esquema ligero, conectarse al UAA y pedir la validación del token de acceso, con lo cual podrían atender la petición del cliente.

\subsection{Implementación y pruebas}

La decisión de implementación fue principalmente que cada uno de los componentes pueda ser ejecutado en una variedad de equipos y con la menor interdependencia, para lo cual se procedió a trabajar en una ar- 
quitectura de microservicios que permita su despliegue sea como procesos independientes, o dentro de una estructura de contenedorización, como Docker [30]. Para los servicios y clientes de escritorio se utilizó Java con Spring Boot en general. Para el servicio de mensajería central se decidió trabajar utilizando el protocolo MQTT y para el desarrollo del bróker se tomó como base la biblioteca Moquette [31], a la cual se modificó para añadirle soporte para OAuth2 principalmente. Para los servicios de persistencia se utilizó como base un clúster HDFS [32] en la red local, y sobre él se construyeron servicios de series de tiempo, que, de acuerdo con los intereses actuales del proyecto, eran los más adecuados para procesar los datos en proveniencia de los sensores. Se interactuó con OpenTSDB [33] y Prometheus [34].

En cuanto a los clientes, se decidió construir bibliotecas genéricas para distintos tipos de sistemas, las cuales faciliten el proceso de desarrollar las aplicaciones específicas. Se desarrolló una biblioteca cliente Java, una para móviles Android, una para Arduino MKR [35] y otra para ESP32 [36], estas tres últimas basadas en Eclipse Paho [37]. Las bibliotecas de Arduino fueron orientadas exclusivamente a su uso en controladores de sensores y actuadores.

Las pruebas del sistema se efectuaron en un ambiente de oficina controlado. El equipo principal fue un RPi 3B + que hizo las veces de Gateway wifi, brindando entre otros los servicios DNS y NTP. Se utilizaron simultáneamente controladores MKR 1010 y ESP32, los cuales recibían en permanencia información de sensores de temperatura, humedad y ruido, como el DHT11 y el KY038 [38]. El RPi3B+ también acogió los servicios REG, UAA y el bróker de mensajería MQTT. Los servicios de persistencia sobre HDFS, así como los de TSDB se instalaron en Linux sobre un i5-4210U con 8 GB de RAM. En este último equipo se instalaron también servicios genéricos para envío y recepción de mensajes, con los cuales se verificó la fluidez de la interacción. Como cliente móvil se utilizó un N9005, que tuvo dos funciones: sensor bobo, enviando en gran cantidad números aleatorios al sistema, y actuador ligero, avisando cada vez que las mediciones de los sensores reales sobrepasaban ciertos niveles parametrizados en la app. En la Figura 4 se presenta uno de los setup del sistema de pruebas.

En la experimentación relativa a las pruebas de seguridad, se decidió asumir que un posible atacante se encontraba ya conectado a la red local y que estaba, potencialmente, en la capacidad de hacer cualquier petición y capturar todo el tráfico. En el primer test se utilizó $Z a p$ para efectuar un scanning tanto activo como pasivo, y se verificó que la seguridad se mantenía (tráfico encriptado), salvo en el caso (documentado en la arquitectura) del esquema ligero.

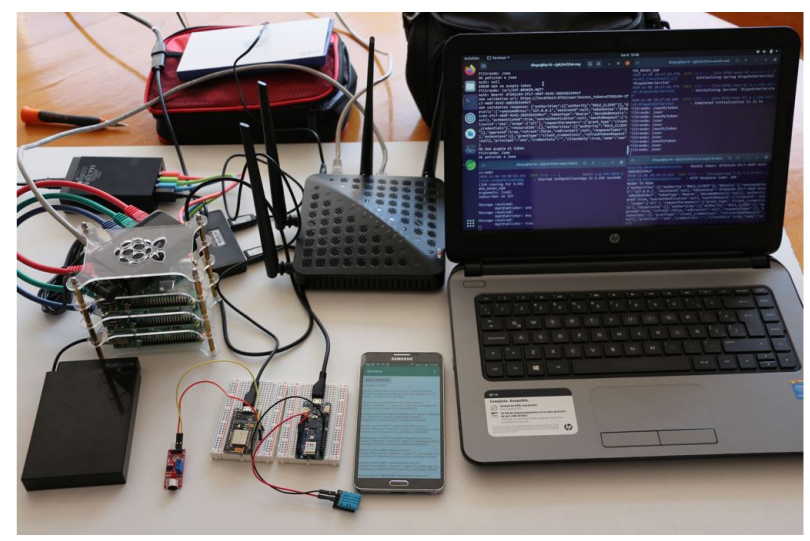

Figura 4. Setup de pruebas: de izquierda a derecha, se observa un clúster de 3 Raspberry Pi ejecutando todos los servicios sobre Docker; un ESP8266 monitoreando nivel de ruido con un KY038; un MKR1010 monitoreando temperatura ambiente con un DHT11; un N9005 inyectando mensajes aleatorios, y una laptop monitoreando todos los servicios.

Luego, se utilizó Wireshark para una verificación más bien de corte manual, analizando los paquetes por tipo de conexión o esquema, y nuevamente se validó, como esperado, que, salvo el esquema ligero, todo el tráfico se mantenía cifrado, como se presenta en la Figura 5.

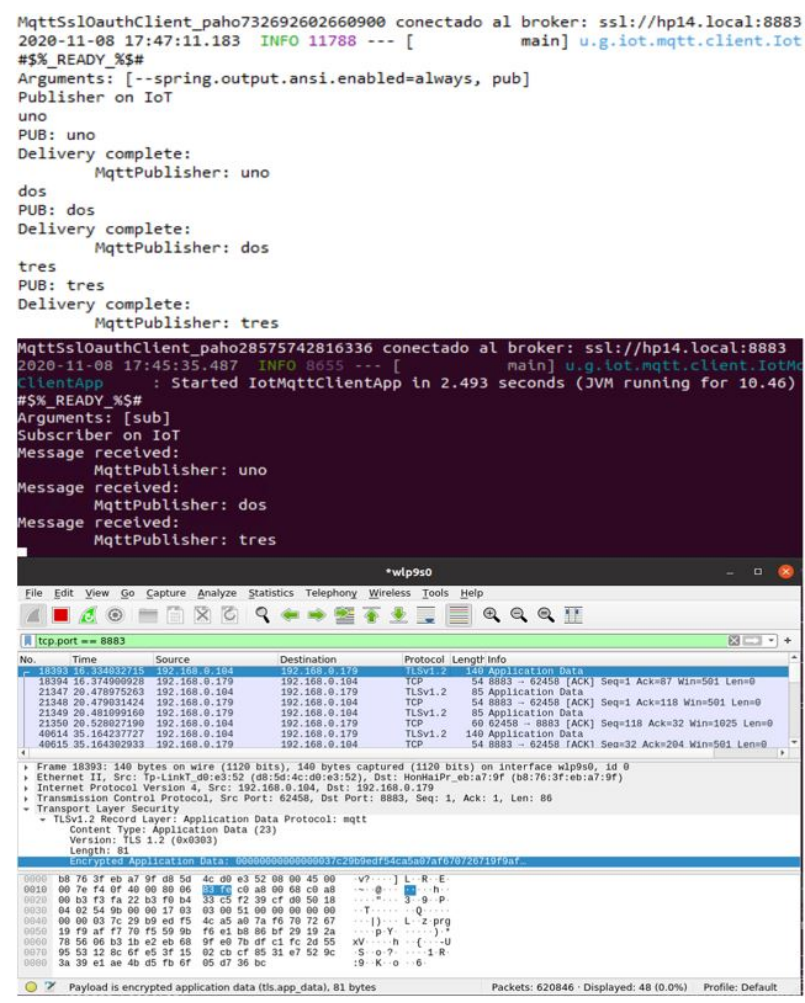

Figura 5. Ejecución y trazas: de arriba hacia abajo, primero, un cliente enviando tres mensajes simples de texto; luego el receptor obtiene los mensajes y, finalmente, la traza de Wireshark que captura los paquetes y verifica que, en tránsito, se encuentran cifrados. 


\section{Discusión}

El proceso de incluir esquemas de seguridad complejos en dispositivos ligeros de IoT, no es trivial como ya lo afirmaron Khan y Salah [8] y en este trabajo se coincide con esta afirmación. Dos casos destacables fueron que el manejo de TLS con certificados autogenerados para MKR 1010 requirió regenerar todo el firmware para incluir la CA, y que no se pudo llevar a cabo en controladores ESP8266 debido a la indisponibilidad, en la práctica, de bibliotecas open-source suficientemente completas para garantizar el nivel de seguridad esperado.

El enfoque adoptado retoma en general las advertencias hechas por Lin y Bergmann [13] e intenta proporcionar un sistema suficientemente completo y sencillo para que con mínimo soporte técnico pudiera ser implementado en el hogar, y luego administrado directamente por el usuario.

Esto, claro, presenta limitaciones dadas por la gran variedad de tipos de usuarios que pueden desear incluirse en el IoT. Sin embargo, las pruebas realizadas apuntan a que el corazón del prototipo permitiría proporcionar esto, si se logran incluir algunas facilidades a nivel de interfaz de usuario, equipos e instaladores.

Este trabajo confirma lo ya expuesto por Ayed et al., [14] y por Fernández et al., [15] en relación con el problema del escalamiento de identidades y el beneficio que puede obtenerse tanto con OAuth como con un enfoque por servicios. Delegar en un punto único la autenticación, confiando luego en credenciales temporales, como lo permite OAuth2, mantiene la infraestructura de identidades ligera, mientras que la distribución en servicios brinda una gran facilidad a la hora de replicar y redundar servicios en clúster cuando la carga de trabajo del sistema así lo amerita. Es justamente este enfoque de servicios / microservicios lo que permite que trabajar con TLS no sea demasiado exigente a nivel de mantenimiento, como destacaron Díaz-Sánchez et al. [20].

Esta propuesta reconoce la importancia que a nivel de seguridad debe darse a los equipos de borde y siguiendo la línea de Shapsough et al., [27] y de Sciancalepore et al., [26] refuerza especialmente el equipo gateway para luego poder implementar mejoras en el protocolo MQTT, al estilo de lo realizado por Singh et al., [28] pero principalmente incluyendo el uso de OAuth2 y TLS.

\section{Conclusiones}

Este trabajo se motivó en una necesidad actual acuciante: asegurar los sistemas de IoT, especialmente aquellos ligados a un contexto hogareño, y hacerlo sin menoscabar la libertad de acceso del usuario para recopilar la información y para modificar las configuraciones de su sistema. Esta necesidad, como se vio, parte entre otras cosas de la relativa informalidad del IoT, especialmente en un entorno de casa inteligente.

Se inició con una concepción metodológica que estratifica el entorno en capas: aquella relacionada más de cerca con la interacción con el espacio mediante recolección de información y ejecución de acciones para modificar el microambiente, y la capa de procesamiento centralizado y análisis de la información. Ambas capas se interrelacionaron con una conexión centralizada que las unifica manteniendo también su acoplamiento ligero.

Esta metodología se orientó a permitir una implementación basada en microservicios, donde, además de evitar en lo posible cualquier tipo de estructura monolítica, se proporcionó un grupo de servicios y bibliotecas para clientes que facilitan en buena medida la generación de nuevas utilidades y componentes. Las pruebas realizadas con los servicios, clientes y sensores generados bajo esta infraestructura permitieron confirmar tanto la solidez como la relativa facilidad de uso de los componentes.

El punto principal, la seguridad, si bien no fue el más fácil de implementar, una vez depurados los tres esquemas definidos para los diferentes tipos de conexiones, demostró ser una elección robusta, que resistió las pruebas de acceso indebido. Cabe indicar, sin embargo, que un esquema metódico de pruebas específico para el campo resta aún por diseñarse y aplicarse, lo cual será motivo de trabajos futuros. Podemos, empero, afirmar que la combinación TLS, OAuth2, MQTT, produjo los resultados esperados en gran medida.

Como principales contribuciones tenemos la implementación de una sólida arquitectura segura de IoT para el hogar; la combinación estable y fluida de al menos tres tecnologías de alto nivel para el manejo de seguridad y una implementación de referencia funcional que podrá ser puesta disposición públicamente para su libre uso.

\section{Referencias}

[1] Y. Lu and L. D. Xu, "Internet of things (IoT) cybersecurity research: A review of current research topics," IEEE Internet of Things Journal, vol. 6, no. 2, pp. 2103-2115, 2019. [Online]. Available: https://doi.org/10.1109/JIOT.2018.2869847

[2] A. Riahi Sfar, E. Natalizio, Y. Challal, and Z. Chtourou, "A roadmap for security challenges in the internet of things," Digital Communications and Networks, vol. 4, no. 2, pp. 118-137, 2018. [Online]. Available: https://doi.org/10.1016/j.dcan.2017.04.003

[3] P. Lea, Internet of Things for Architects: Architecting IoT solutions by implementing sensors, communication infrastructure, edge computing, 
analytics, and security. Packt Publishing Ltd, 2018. [Online]. Available: https://bit.ly/3oJ1XRl

[4] P. Jamshidi, C. Pahl, N. C. Mendonça, J. Lewis, and S. Tilkov, "Microservices: The journey so far and challenges ahead," IEEE Software, vol. 35, no. 3, pp. 24-35, 2018. [Online]. Available: https://doi.org/10.1109/MS.2018.2141039

[5] J. Khan, J. p. Li, I. Ali, S. Parveen, G. a. Khan, M. Khalil, A. Khan, A. U. Haq, and M. Shahid, "An authentication technique based on oauth 2.0 protocol for internet of things (IoT) network," in 2018 15th International Computer Conference on Wavelet Active Media Technology and Information Processing (ICCWAMTIP), 2018, pp. 160-165. [Online]. Available: https: //doi.org/10.1109/ICCWAMTIP.2018.8632587

[6] C. Chan, R. Fontugne, K. Cho, and S. Goto, "Monitoring tls adoption using backbone and edge traffic," in IEEE INFOCOM 2018 IEEE Conference on Computer Communications Workshops (INFOCOM WKSHPS), 2018, pp. 208-213. [Online]. Available: https: //doi.org/10.1109/INFCOMW.2018.8406957

[7] F. Izquierdo, M. Ciurana, F. Barcelo, J. Paradells, and E. Zola, "Performance evaluation of a TOA-based trilateration method to locate terminals in WLAN," in 2006 1st International Symposium on Wireless Pervasive Computing, 2006, pp. 1-6. [Online]. Available: https://doi.org/10.1109/ISWPC.2006.1613598

[8] M. A. Khan and K. Salah, "IoT security: Review, blockchain solutions, and open challenges," Future Generation Computer Systems, vol. 82, pp. 395-411, 2018. [Online]. Available: https://doi.org/10.1016/j.future.2017.11.022

[9] J. P. Rojas, J. C. Bustos, and D. Ordóñez Camacho, "Smart public transportation at your fingertips," Enfoque UTE, vol. 8, no. 1, pp. 122-134, Feb. 2017. [Online]. Available: https://doi.org/10.29019/enfoqueute.v8n1.143

[10] J. P. Rojas, J. C. Bustos, and D. OrdóñezCamacho, "Qbus: Movilidad inteligente para el usuario de transporte público," in Proceedings of the International Conference on Information Systems and Computer Science, INCISCOS 2016, 2016. [Online]. Available: https://bit.ly/3jZlBpE

[11] E. A. Q. Montoya, S. F. J. Colorado, W. Y. C. Muñoz, and G. E. C. Golondrino, "Propuesta de una arquitectura para agricultura de precisión soportada en IoT," RISTI - Revista
Iberica de Sistemas e Tecnologias de Informacao, pp. 39-56, 2017. [Online]. Available: http://dx.doi.org/10.17013/risti.24.39-56

[12] M. Agiwal, N. Saxena, and A. Roy, "Towards connected living: 5g enabled internet of things (IoT)," IETE Technical Review, vol. 36, no. 2, pp. 190-202, 2019. [Online]. Available: https://doi.org/10.1080/02564602.2018.1444516

[13] H. Lin and N. Bergmann, "IoT privacy and security challenges for smart home environments," Information, vol. 7, no. 3, p. 44, Jul 2016. [Online]. Available: http://dx.doi.org/10.3390/info7030044

[14] H. Kaffel-Ben Ayed, H. Boujezza, and I. Riabi, "An idms approach towards privacy and new requirements in IoT," in 2017 13th International Wireless Communications and Mobile Computing Conference (IWCMC), 2017, pp. 429-434. [Online]. Available: https://doi.org/10.1109/IWCMC.2017.7986324

[15] F. Fernández, A. Alonso, L. Marco, and J. Salvachúa, "A model to enable applicationscoped access control as a service for IoT using OAuth 2.0," in 2017 20th Conference on Innovations in Clouds, Internet and Networks (ICIN), 2017, pp. 322-324. [Online]. Available: https://doi.org/10.1109/ICIN.2017.7899433

[16] J. Bugeja, A. Jacobsson, and P. Davidsson, "On privacy and security challenges in smart connected homes," in 2016 European Intelligence and Security Informatics Conference (EISIC), 2016, pp. 172-175. [Online]. Available: https://doi.org/10.1109/EISIC.2016.044

[17] L. Sun, Y. Li, and R. A. Memon, "An open IoT framework based on microservices architecture," China Communications, vol. 14, no. 2, pp. 154-162, 2017. [Online]. Available: https://doi.org/10.1109/CC.2017.7868163

[18] T. Vresk and I. Çavrak, "Architecture of an interoperable IoT platform based on microservices," in 2016 39th International Convention on Information and Communication Technology, Electronics and Microelectronics (MIPRO), 2016, pp. 1196-1201. [Online]. Available: https://doi.org/10.1109/MIPRO.2016.7522321

[19] R. Yu, V. T. Kilari, G. Xue, and D. Yang, "Load balancing for interdependent IoT microservices," in IEEE INFOCOM 2019 IEEE Conference on Computer Communications, 2019, pp. 298-306. [Online]. Available: https: //doi.org/10.1109/INFOCOM.2019.8737450 
[20] D. Díaz-Sánchez, A. Marín-Lopez, F. A. Mendoza, P. A. Cabarcos, and R. S. Sherratt, "TLS/PKI challenges and certificate pinning techniques for IoT and M2M secure communications," IEEE Communications Surveys Tutorials, vol. 21, no. 4, pp. 3502-3531, 2019. [Online]. Available: https://doi.org/10.1109/COMST.2019.2914453

[21] P. Urien, "Securing the IoT with TLS/DTLS server stacks embedded in secure elements: An ePlug usecase," in 2017 14th IEEE Annual Consumer Communications Networking Conference (CCNC), 2017, pp. 569-570. [Online]. Available: https://doi.org/10.1109/CCNC.2017.7983170

[22] J. D. Hoz, J. Saldana, J. FernándezNavajas, J. Ruiz-Mas, R. G. Rodríguez, and F. d. J. M. Luna, "SSH as an alternative to TLS in IoT environments using HTTP," in 2018 Global Internet of Things Summit (GIoTS), 2018, pp. 1-6. [Online]. Available: https://doi.org/10.1109/GIOTS.2018.8534545

[23] M. Khan, M. W. Anwar, F. Azam, F. Samea, and M. F. Shinwari, A Model-Driven Approach for Access Control in Internet of Things (IoT) Applications - An Introduction to UMLOA. Communications in Computer and Information Science, Springer, 2018, vol. 920. [Online]. Available: https://doi.org/10.1007/978-3-319-99972-2_16

[24] H. Kim, A. Wasicek, B. Mehne, and E. A. Lee, "A secure network architecture for the internet of things based on local authorization entities," in 2016 IEEE 4th International Conference on Future Internet of Things and Cloud (FiCloud), 2016, pp. 114-122. [Online]. Available: https://doi.org/10.1109/FiCloud.2016.24

[25] M. Pahl and L. Donini, "Securing IoT microservices with certificates," in NOMS 2018 - 2018 IEEE/IFIP Network Operations and Management Symposium, 2018, pp. 1-5. [Online]. Available: https://doi.org/10.1109/NOMS.2018.8406189

[26] S. Sciancalepore, G. Piro, D. Caldarola, G. Boggia, and G. Bianchi, "Oauth-iot: An access control framework for the internet of things based on open standards," in 2017 IEEE Symposium on Computers and Communications (ISCC), 2017, pp. 676-681. [Online]. Available: https://doi.org/10.1109/ISCC.2017.8024606

[27] S. Shapsough, F. Aloul, and I. A. Zualkernan, "Securing low-resource edge devices for
IoT systems," in 2018 International Symposium in Sensing and Instrumentation in IoT Era (ISSI), 2018, pp. 1-4. [Online]. Available: https://doi.org/10.1109/ISSI.2018.8538135

[28] M. Singh, M. A. Rajan, V. L. Shivraj, and P. Balamuralidhar, "Secure mqtt for internet of things (IoT)," in 2015 Fifth International Conference on Communication Systems and Network Technologies, 2015, pp. 746-751. [Online]. Available: https://doi.org/10.1109/CSNT.2015.16

[29] C. Singh and M. Kumar, Mastering Hadoop 3: Big data processing at scale to unlock unique business insights. Packt Publishing, 2019. [Online]. Available: https://bit.ly/37Qi2O9

[30] J. Turnbull, The Docker Book: Containerization is the new virtualization, 2014. [Online]. Available: https://bit.ly/3m7nqRY

[31] A. Selva. (2014) Java MQTT lightweight broker. moquette. [Online]. Available: https: //bit.ly/3gB82Mw

[32] M. Bhushan, Big Data and Hadoop: Learn by Example. BPB Publications, 2018. [Online]. Available: https://bit.ly/2W0AmP1

[33] T. Dunning and E. Friedman, Time Series Databases: New Ways to Store and Access Data, Edition: 1. Sebastopol. O'Reilly Media, Inc, 2014. [Online]. Available: https://bit.ly/2W1VnsU

[34] B. Brazil, Prometheus: Up \& Running: Infrastructure and Application Performance Monitoring. O'Reilly Media, 2018. [Online]. Available: https://bit.ly/39V80xX

[35] A. Kurniawan, Arduino MKR WIFI 1010 Development Workshop. PE Press, 2018. [Online]. Available: https://bit.ly/37OEnvD

[36] I. Dogan and I. Ahmet, The Official ESP32 Book. Elektor International Media, 2017. [Online]. Available: https://bit.ly/2IzEW3G

[37] G. C. Hillar, Hands-On MQTT Programming with Python: Work with the lightweight IoT protocol in Python. Packt Publishing, 2018. [Online]. Available: https://bit.ly/33YpdTg

[38] B. Charles, Beginning Sensor Networks with Arduino and Raspberry Pi. Apress, 2013. [Online]. Available: https://bit.ly/3m5syGj 


\title{
ANÁLISIS DE VULNERABILIDADES CON SQLMAP APLICADA A ENTORNOS APEX 5 VULNERABILITY ANALYSIS WITH SQLMAP APPLIED TO APEX5 CONTEXT
}

\author{
Esteban Crespo-Martínez ${ }^{1, *}$
}

Recibido: 14-09-2020, Revisado: 01-10-2020, aprobado tras revisión: 30-11-2020

\section{Resumen}

Las bases de datos son usualmente los principales objetivos de un ataque, específicamente por la información que en ella reside, ya que, de acuerdo con Druker, la información es poder. En este trabajo se realizan las pruebas de vulnerabilidad de la base de datos de un software ERP desarrollado en APEX 5. Para ello, se utilizan herramientas FOSS de prueba y análisis de vulnerabilidades de bases de datos, identificando que las sesiones que utiliza ERP basada en Oracle APEX son realizadas de manera aleatoria y que, además, son nuevamente generadas en determinados momentos. Se concluye que, con las pruebas aplicadas y las actualizaciones de SQLMAP a la fecha del experimento, no se ha conseguido vulnerar el software ERP con técnicas de inyección SQL.

Palabras clave: APEX, evaluación a sistemas de información, inyección SQL, protección de datos

\begin{abstract}
Databases are usually the main targets of an attack, specifically for the information that they store, since, according to Druker, information is power. In this work vulnerability tests are performed of the database of an ERP software developed in APEX 5. For this purpose, FOSS tools are used to test and analyze vulnerabilities of databases, identifying that sessions used by ERP based on Oracle APEX are carried out randomly, and besides are generated again at particular times. It is therefore concluded that, with the tests applied and the updates of SQLMAP to the date of the experiment, it has not been possible to vulnerate the ERP software with SQL injection techniques.
\end{abstract}

Keywords: APEX, Data protection, Information systems evaluation, SQL Injection.

\footnotetext{
1,* Universidad del Azuay, Ecuador. Autor para correspondencia ${ }^{\star}$ : ecrespo@uazuay.edu.ec.

(D) https://orcid.org/0000-0002-3061-9045
} 


\section{Introducción}

Varios expertos en seguridad de la información coinciden con que los ataques informáticos cada vez son más recurrentes, y usualmente a los sistemas web, alterando o exponiendo la información personal [1], en especial a las aplicaciones web [2], debido a su complejidad, extensión, alta personalización y usualmente desarrollados por programadores con poca experiencia en seguridad [3].

No se puede negar que, en esta sociedad de la información y el conocimiento, las bases de datos contienen la mina de oro, esta se convierte en uno de los elementos estratégicos más importantes de la organización, pues de su análisis e interpretación en el momento oportuno se pueden proyectar estrategias para aventajar oportunidades y prever amenazas según el rol de la organización en la sociedad.

La protección de la información toma sentido cuando aparecen los primeros virus informáticos: alteraban o borraban la información del usuario, muchas veces con fines de demostrar la capacidad destructora y creativa de quien diseñaba el malware para acometerla. El contexto informático era más simple, no existía la intercomunicación entre organizaciones y los sistemas se limitaban a funcionar de manera centralizada [4]. Sin embargo, la explosión de oportunidades que se generan por la introducción de Internet hace que los atacantes vean de otra manera a los datos. Dañarlos o eliminarlos ya no tiene sentido, el robo, la copia o el secuestro son aspectos que se convierten en nuevos objetivos.

Ojagbule et al. [5] mencionan que, hoy en día, existen más de un billón de sitios web, y que muchos de ellos son desarrollados por gestores de contenido como Drupal, Joomla o WordPress, y que, según Mohammadi \& Namadchian [6], contienen datos importantes.

Según Ojagbule et al. [5] y Kruegel et al. [6], al existir una gran cantidad de sitios, también existe una gran cantidad de bases de datos que están expuestas a vulnerabilidades y riesgos. De esto aparece una técnica conocida como inyección SQL (Structured Query Language Injection Attack por sus siglas en inglés SQLIA), que según Santin, Oliveira y Lago [7] citando a [8] y [9], es una técnica donde un atacante explora vulnerabilidades que permiten alterar los comandos SQL en una aplicación y que es conocida como una de las vulnerabilidades que generan mayor impacto en la organización.

Nofal y Amber [9] agregan que esta técnica usualmente no tiene patrones predecibles o específicos, lo que se convierte en un problema importante para investigadores y desarrolladores. Concluye Badaruddin [10] que la técnica de inyección SQL es el segundo error más común encontrado en los servidores web en Internet con alrededor del 44,11\%.
Con el propósito de descubrir fallas de seguridad en cuanto a vulnerabilidades de inyección SQL, en este trabajo se realiza una evaluación con SQLMAP a una base de datos Oracle que almacena la información del sistema UDA-ERP desarrollado en APEX 5 por la Universidad del Azuay. Este artículo se divide en las siguientes secciones: i) el estado del arte, donde se establecen algunos conceptos, así como también los trabajos relacionados; ii) la metodología aplicada para la obtención de los resultados, detallando las configuraciones realizadas en el equipo de laboratorio de pruebas; iii) los resultados obtenidos tras la ejecución de la herramienta; iv) la discusión sobre los resultados adquiridos y v) las conclusiones y trabajos futuros.

\subsection{La inyección SQL}

Según OWASP, la inyección SQL es una de las diez vulnerabilidades más peligrosas y populares que pueden presentarse en entornos web [11], los cuales generalmente son difíciles de proteger debido a su alta personalización, complejidad, escala [3], tecnología y desarrollo por programadores con poca experiencia en seguridad $[3,12]$, causando serios daños a los negocios de las víctimas [13]. Sumado a esto, Setiawan y Setiyadi [14] sostienen que, en un contexto de redes de computadoras, cualquier dato existente en un computador conectado a otro se vuelve inseguro.

Los autores Santin, Oliveira y Lago [7] citando a [15] mencionan que no existen soluciones que garanticen o solucionen todas las vulnerabilidades, las cuales ocurren a nivel de hardware y software, expresión a la que se suma Setiawan [14]. Agregan que, como muchos elementos no son actualizados constantemente, son más propensos a ataques cibernéticos. Por otro lado, Kals et al. [12] sostienen que son múltiples las vulnerabilidades de seguridad de aplicaciones web, resultado de problemas genéricos de validación de entrada. Además, las vulnerabilidades pueden mantenerse secretas o reportadas por los fabricantes, sea de forma pública o privada [16].

De forma básica, un ataque de inyección SQL puede ser representado como se indica en la Figura 1.

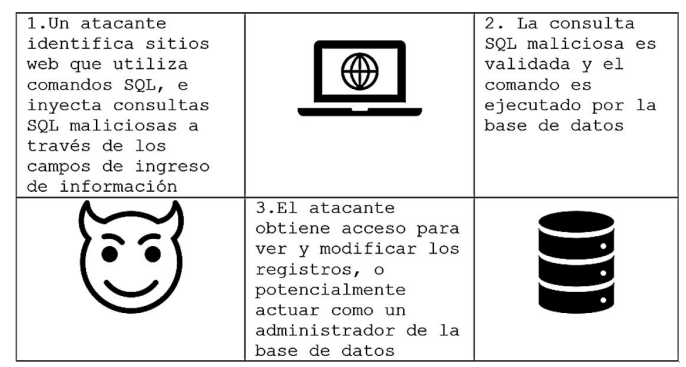

Figura 1. Proceso de la inyección SQL

Otra forma de representar la secuencia de ataques SQL es la que propone AVI Networks [17], la cual se presenta en la Figura 2. 


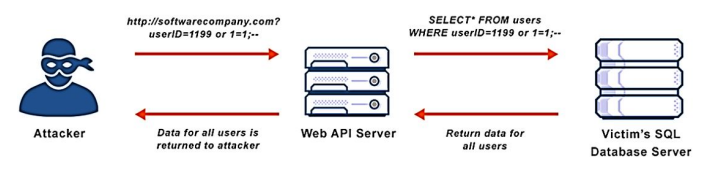

Figura 2. Secuencia de ataque SQL Injection [17]

De acuerdo con [18], las técnicas de ataque de inyección SQL pueden clasificarse de la siguiente manera:

i) Tautologías, un tipo de ataque que utiliza consultas condicionales e inserta tokens SQL en ellas, demostrando siempre ser cierto.

ii) Consultas ilegales o lógicamente incorrectas, donde los atacantes utilizan los mensajes de error de las bases de datos para encontrar vulnerabilidades en las aplicaciones.

iii) Consultas con UNION, donde los atacantes inyectan consultas infectadas sobre consultas seguras utilizando el operador UNION y, por lo tanto, recuperar información de la base de datos.

iv) Consultas con respaldo o Piggy-backed: los atacantes adjuntan delimitadores como ";" a la consulta original y las ejecutan simultáneamente, siendo la primera legítima y las demás falsas, pero retornando información valiosa.

v) Procedimientos almacenados (Stored procedures), un subconjunto de consultas precompiladas, dependiendo de cuales sean existirán diferentes formas de ataque.

vi) Inyección ciega o Blind Injection, en la que los desarrolladores ocultan mensajes de error que pueden ser útiles para los atacantes para planificar y ejecutar un ataque SQLIA. En esta situación el atacante se encuentra con una página estática, donde realiza preguntas verdaderas y falsas mediante el uso de comandos SQL hasta conseguir el objetivo.

vii) Ataques temporizados, que permiten al atacante observar el tiempo requerido para ejecutar una consulta. El atacante genera una consulta grande utilizando sentencias if-else y, de esta manera, medir la cantidad de tiempo que tarda la página en cargarse para determinar si la sentencia inyectada es verdadera.

viii) Codificaciones alternativas, donde codificaciones $A S C I I$ y Unicode permiten evadir el filtro que escanea "caracteres especiales" [19].
Una evaluación de vulnerabilidades por inyección SQL puede ser realizada con el uso de herramientas tecnológicas para tal propósito. Novaski [20] sugiere el uso de herramientas FOSS, de las cuales propone 14 para ser utilizadas: Arachni, Beef, Htcap, IronWASP, Metasploit, Skipfish, SQLMap, Vega, W3af, Wapiti, Wfuzz, XSSer, Xenotix y ZAP.

De este trabajo agrega que solo las herramientas IronWASP, Vega, ZAP y SQLMap detectaron la vulnerabilidad de inyección SQL, mientras que la vulnerabilidad XSS reflejada solo fue detectada por las herramientas ZAP y Xenotix.

Indica en su trabajo que solo fue posible realizar una prueba de intrusión completa en la vulnerabilidad de inyección SQL, y para realizarla fue necesario aplicar tres herramientas diferentes: i) wapiti-getcookie, para obtener el identificador de sesión; ii) Htcap para obtener puntos de entrada; y SQLMap para detectar y explotar la vulnerabilidad.

Entre los trabajos relacionados están los que se encuentran en la Tabla 1. Este trabajo, a diferencia de los citados, hace énfasis en probar aspectos de seguridad en una aplicación desarrollada en Oracle APEX 5 .

Está claro que, a pesar del tiempo transcurrido desde la primera vez que apareció el ataque de inyección SQL hace dos décadas [21], son numerosas las técnicas de inyección, así como las técnicas de evasión y mitigación. Las tecnologías de información están cada vez más frecuentes en nuestro entorno y han afectado notablemente el estilo de vida, pues cada vez que aumenta el uso y la confiabilidad en las computadoras y los sistemas informáticos, la amenaza a los datos sensibles también aumenta.

Las vulnerabilidades de inyección SQL en las aplicaciones web son sorprendentemente vastas y, definitivamente, son una gran amenaza para la seguridad de los datos personales que se almacenan en la web [21].

En la práctica, Cetin et al. [22] demuestran que un análisis automatizado de GitHub enseña que el 15,7 \% de los 120412 archivos fuente de Java publicados contienen código vulnerable a los ataques de inyección de identificador de SQL (SQL-IDIA) señalando, además que, tras una revisión manual, comprobaron que 18939 archivos Java identificados durante el análisis automatizado son vulnerables a este tipo de ataques.

Puneet [23] clasifica a la inyección SQL en dos tipos: i) la inyección SQL clásica y ii) la inyección SQL avanzada. 
Tabla 1. Trabajos relacionados con el uso de SQLMap

\begin{tabular}{|c|c|c|c|c|}
\hline Id. & Autores & Tema & Año & Objetivo \\
\hline 1 & $\begin{array}{l}\text { Nofal D.E. } \\
\text { Amer A. A. [9] }\end{array}$ & $\begin{array}{l}\text { SQL Injection } \\
\text { Attacks } \\
\text { Detection and } \\
\text { Prevention } \\
\text { Based on Neuro- } \\
\text { Fuzzy } \\
\text { Technique }\end{array}$ & 2020 & $\begin{array}{l}\text { Realizan un trabajo para detectar y } \\
\text { prevenir ataques de inyección } \mathrm{SQL} \text {, } \\
\text { aplicando un sistema de inferencia de } \\
\text { lógica difusa. }\end{array}$ \\
\hline 2 & $\begin{array}{l}\text { O. Ojagbule } \\
\text { H. Wimmer } \\
\text { R. Haddad [24] }\end{array}$ & $\begin{array}{l}\text { Vulnerability } \\
\text { Analysis of } \\
\text { Content } \\
\text { Management } \\
\text { Systems to SQL } \\
\text { Injection Using }\end{array}$ & 2018 & $\begin{array}{l}\text { Comparan las vulnerabilidades de } \\
\text { inyección SQL en tres gestores de } \\
\text { contenido más utilizados, considerando } \\
\text { las herramientas Nikto y SQLMap para } \\
\text { tal propósito. }\end{array}$ \\
\hline 3 & $\begin{array}{l}\text { F. Santin } \\
\text { J. A. Oliveira V. } \\
\text { Lago Machado [7] }\end{array}$ & $\begin{array}{l}\text { Uso da } \\
\text { ferramenta } \\
\text { SQLMap para } \\
\text { detecção de } \\
\text { vulnerabilidades } \\
\text { de SQL } \\
\text { Injection }\end{array}$ & 2017 & $\begin{array}{l}\text { Se enfoca a exponer los principales } \\
\text { riesgos a los que se someten las } \\
\text { aplicaciones web relacionadas con la } \\
\text { inyección SQL. Hacen uso de la } \\
\text { herramienta SQLMap para tal } \\
\text { propósito. }\end{array}$ \\
\hline 4 & $\begin{array}{l}\text { Badaruddin Bin Halib, } \\
\text { Edy Budiman, Hario } \\
\text { Jati Setyadi [10] }\end{array}$ & $\begin{array}{l}\text { Técnicas de } \\
\text { pirateo de } \\
\text { servidores web } \\
\text { con SQLMap en } \\
\text { Kali Linux }\end{array}$ & 2017 & $\begin{array}{l}\text { Proponen una técnica de hacking a } \\
\text { servidores web mediante el uso de } \\
\text { SQLMap en Kali Linux. }\end{array}$ \\
\hline 5 & S. D. Axinte [25] & $\begin{array}{l}\text { SQL injection } \\
\text { Testing in Web } \\
\text { Applications } \\
\text { Using SQLMap }\end{array}$ & 2014 & $\begin{array}{l}\text { La autora realiza un análisis analítico de } \\
\text { la técnica de inyección SQL, y presenta } \\
\text { métodos, herramientas y acciones de } \\
\text { prevención. }\end{array}$ \\
\hline 6 & $\begin{array}{l}\text { Barinas, Alarcón, } \\
\text { Callejas [1] }\end{array}$ & $\begin{array}{l}\text { Vulnerabilidad } \\
\text { de ambientes } \\
\text { virtuales de } \\
\text { aprendizaje } \\
\text { utilizando } \\
\text { SQLMap, RIPS, } \\
\text { W3AF y Nessus* }\end{array}$ & 2014 & $\begin{array}{l}\text { Trabajo en el que analizan los aspectos } \\
\text { de seguridad de ambientes virtuales de } \\
\text { aprendizaje, herramientas de seguridad y } \\
\text { análisis de vulnerabilidades. }\end{array}$ \\
\hline 7 & $\begin{array}{l}\text { A. Tajpour, } \\
\text { S. Ibrahim, } \\
\text { M. Masrom [26] }\end{array}$ & $\begin{array}{l}\text { SQL Injection } \\
\text { Detection and } \\
\text { Prevention } \\
\text { Techniques }\end{array}$ & 2015 & $\begin{array}{l}\text { Proponen técnicas de ataque y } \\
\text { mitigación frente a ataques de inyección } \\
\text { SQL, comparando varios tipos de ellos. }\end{array}$ \\
\hline
\end{tabular}

\subsubsection{Inyección SQL clásica}

Las técnicas de inyección básica, sugeridas por [23] se resumen en las siguientes:

\section{a) Piggy Backed Queries}

La intención del ataque es primordialmente la denegación de servicio. La base de datos recibe múltiples consultas, en las que, durante la ejecución, la consulta normal funciona como en un caso normal, mientras que la segunda consulta se adhiere a la primera para conseguir el ataque. Un ejemplo de este ataque puede ser el siguiente:

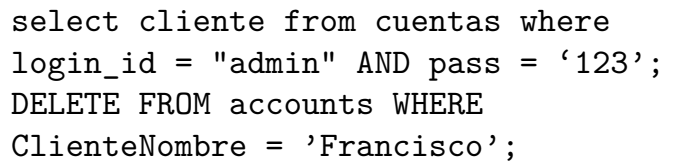


Posterior a la ejecución de la primera consulta, el intérprete detecta el punto y coma ";" y ejecuta la segunda consulta junto con la primera, eliminando todos los datos del cliente "Francisco". Este tipo de datos maliciosos pueden protegerse determinando en primer lugar la consulta SQL correcta mediante la validación adecuada o utilizando adecuadas técnicas de detección, como es el análisis estático, que no necesita la supervisión del tiempo de ejecución.

\section{b) Stored procedure}

La intención de ataque se resume en autenticación de escape y en denegación de servicio. Erradamente, los profesionales de TI piensan que los procedimientos almacenados de SQL son un remedio para la inyección de SQL [17], ya que se los coloca el frente de las bases de datos y las características de seguridad no son directamente aplicables. Los procedimientos almacenados no usan el lenguaje de consulta estructurado estándar, usa sus propios lenguajes de script que no tienen la misma vulnerabilidad que SQL, pero que mantienen otras diversas vulnerabilidades relacionadas con el lenguaje de scripting. Como ejemplo, se puede indicar lo siguiente:

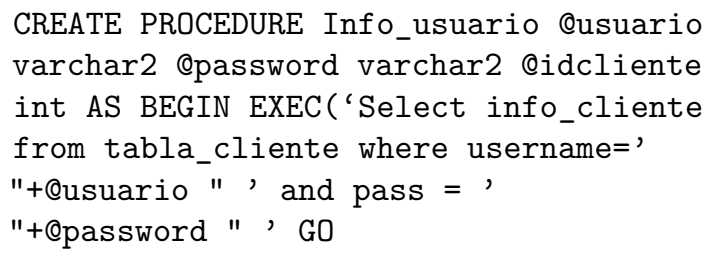

Cualquier usuario malicioso puede ingresar datos maliciosos en los campos del nombre de usuario y password. Un simple comando ingresado podría destruir toda la base de datos o provocar una denegación del servicio. De esta forma, [23] sugiere que no se acopie información crítica en los procedimientos almacenados.

\section{c) Union query}

Es un tipo de ataque que utiliza el operador unión (U) mientras inserta la consulta SQL. Las dos consultas SQL, la normal y la nociva, son unidas mediante este operador. El ejemplo muestra cómo se puede proceder, visualizando que la segunda consulta es maliciosa y no se tiene en cuenta el siguiente texto (-), ya que se convierte en comentario para el Analizador de SQL.

select * from cuentas where id='212'

UNION select * from factura where

usuario='admin'-' and password='pass'

\subsection{2. d) Codificación alternativa}

Con respecto a este tipo de ataque, el atacante cambia el patrón de la inyección SQL para que no sea detectado por las técnicas comunes de detección y prevención. En este método, el atacante utiliza la representación de código hexadecimal, Unicode, octal y ASCII en la instrucción SQL, evitando ser detectados debido al uso de cadenas codificadas.

\subsubsection{Inyección SQL avanzada}

Las técnicas de inyección SQL avanzada, sugeridas por [23] se resumen en las siguientes:

\section{a) Inyección $\mathrm{SQL}$ a ciegas o Deep Blind $S Q L$ Injection Attack}

Una gran cantidad de aplicaciones web se deshabilitan la visualización de errores MYSQL u otro SQL. En este ataque, la información se infiere mediante preguntas de verdadero/falso. Si el punto de inyección es absolutamente ciego, entonces la única forma de atacar es mediante el uso del comando WAIT FOR DELAY o BENCHMARK [23].

\section{b) Fast flux SQL Injection Attack}

El objetivo del ataque es la extracción de datos o la suplantación de identidad mediante phishing. Un host que realiza phishing puede ser detectado fácilmente mediante el seguimiento de su dirección IP o la identificación de su nombre de dominio. Sin embargo, concordando con [23] y [27], los sistemas de protección de muchos hostings web podrían suspender el servicio debido al masivo tráfico que se genera, anulando los propósitos criminales. De esta manera, para evitar este inconveniente, los atacantes aplican la técnica del Fast Flux, que es una técnica DNS para ocultar los sitios de distribución de Phishing y malware detrás de una red de cambio constante.

\subsection{4. c) Ataques de inyección SQL compuestos}

Se trata de una mezcla de dos o más técnicas de ataque, generando un efecto mayor a los indicados con las técnicas anteriormente descritas. Compounded SQL Injection, como es conocido en el mundo oscuro, es derivada de la mezcla del ataque SQL y otros ataques de aplicaciones web, como, por ejemplo, el ataque de inyección SQL + ataques de denegación de servicio distribuidos (DDoS). Sobre la base de lo que expone [23] citando a [28], el código para hacer este tipo de ataque sería:

http://exploitable-web.com/link.php?id=1' union select 1,2,tab1,4 from 
(select decode (encode (convert

(compress (post)

using latin1),

des_encrypt

(concat (post, post, post, post) , 8)),

des_encrypt (sha1 (concat (post,post,

post,post)) ,9))

as tab1 from table_1)a-

Otra forma de combinar un ataque es el de mezclar una inyección SQL con la autenticación insuficiente. Este ataque es explotable cuando los parámetros de seguridad no han sido inicializados donde la aplicación falla al identificar la ubicación del usuario, el servicio o la aplicación. Esto permite al atacante acceder a información privilegiada sin la verificación de la identidad del usuario.

De esta manera, este tipo de ataque es relativamente más sencillo que con cualquier otro tipo de ataque [23], donde el primer paso es ubicar un sitio web que tenga autenticación insuficiente.

\section{Materiales y métodos}

El propósito del análisis de seguridad fue el de evaluar la seguridad de una aplicación desarrollada en Oracle APEX, plataforma en la que está desarrollado el software UDA ERP de la Universidad del Azuay. Con esta premisa se configuró un laboratorio considerando los materiales y métodos que se exponen a continuación.

Para las pruebas se consideró la suite KALI y se utilizó la herramienta SQLMAP, herramienta basada en Free and Open Source, desarrollada sobre una licencia GNU GPLv2 por Miroslav Stampar y Bernardo Damele, considerando que, según [18], SQLMAP soporta, entre otras, la base de datos Oracle, que es la que utiliza el software UDA ERP.

Acota que SQL soporta seis técnicas de inyección: a ciegas basada en booleana (boolean-based blind), a ciegas temporalizado (time-based blind), basada en error (error-based), basada en consultas UNION (UNION query-based), fuera de banda (out-of-band) y consultas apiladas (stack querys).

Las técnicas anteriormente mencionadas forman parte de los parámetros de testeo que están incluidas en SQLMAP, las cuales se aplican automáticamente. Utilizando BurpSuite, se ajusta la captura de cookies de sesión, aplicando la configuración de un proxy local (127.0.0.1:8080) con el propósito de capturar las peticiones POST, que posteriormente serán utilizadas con SQLMAP. Previo a la ejecución de las pruebas se actualizaron las dependencias y paquetes de la suite.

Reconociendo que SQLMAP es una herramienta que permite explorar servidores de bases de datos, para su uso es importante apuntar a la dirección URL que contiene un script SQL. La estructura "sqlmap -u URL -[parámetros]" es la sentencia común, donde el parámetro -dbs permitirá obtener la base de datos. Posterior a la detección de la vulnerabilidad, se debe usar el parámetro -D y el nombre de la base de datos que será analizada. Si el resultado obtenido es efectivo, el parámetro -tables permitirá recuperar todas las tablas de la base de datos especificada.

Con el propósito de identificar las vulnerabilidades, se realizaron tres pruebas, en cada una se incrementó el análisis y se variaron aspectos como el nivel de agresividad en las pruebas, el uso de cookies de sesiones establecidas y la evasión a sistemas de identificación como elaborarlo.

La primera prueba consistió en listar las bases de datos; en la segunda, se aumentó el grado de agresividad y cantidad de pruebas para obtener información de las bases de datos; y en el tercer ataque se pretendió usar un agente aleatorio evadiendo los proxys con el único propósito de capturar una cookie de sesión, elemento que es usado como base para las pruebas automatizadas, en las que se simula una sesión válida de un usuario activo.

\section{Resultados}

\subsection{Primera prueba}

La evaluación de vulnerabilidades de la base de datos mediante la ejecución del comando root@kali: /sqlmapdev\# python3 sqlmap.py -u -dbs, dio como resultado lo expresado en la Tabla 2, considerando que el ataque generó su propia cookie para evaluación: ('USUARIO=ORA_WWV-cUs...UNNyk6flfB'). La prueba inicia a las 13:03:52 del 2020-03-04 y termina a las 13:04:22 /2020-03-04/

Tabla 2. Resultados de la primera prueba

\begin{tabular}{cc}
\hline Test & Descripción \\
\hline Análisis heurístico & $\begin{array}{c}\text { El análisis heurístico detectó que el } \\
\text { objetivo es protegido por algún tipo } \\
\text { de WAF/IPS. }\end{array}$ \\
\hline Contenido URL & $\begin{array}{c}\text { El parámetro 'p' del método GET } \\
\text { no parece ser dinámico. }\end{array}$ \\
& $\begin{array}{c}\text { Las pruebas heurísticas básicas realizadas } \\
\text { indican que el parámetro 'p' no son } \\
\text { inyectables. }\end{array}$ \\
\hline Inyección SQL & No es vulnerable. \\
\hline Test 'AND boolean-based blind- & $\begin{array}{c}\text { Significa que existen valores "reflexivos" } \\
\text { dentro de la respuesta que contiene } \\
\text { (partes de) la carga útil. Esto es } \\
\text { notablemente malo en algunos casos, } \\
\text { ner HAVING clause' } \\
\text { especialmente, en inyecciones booleanas. }\end{array}$ \\
\hline
\end{tabular}

\subsection{Segunda prueba}

En la segunda prueba se ejecutó el comando con las opciones: python3 sqlmap-dev/sqlmap.py - 
u "http://172.16.1.87:8080/ords/f?p=502" -level $=5$ risk $=3-$ dbs $-\mathrm{a}-$ tamper $=$ between. La prueba inicia a las 12:30 del 2020-03-05 y culmina a las 15:18 del 2020-03-05.

Con los parámetros seleccionados se aumenta la intensidad del ataque, así como el nivel y cantidad de pruebas. Los resultados se expresan en la Tabla 3.

Tabla 3. Resultados de la segunda prueba

\begin{tabular}{|c|c|}
\hline Test & Descripción \\
\hline Análisis heurístico & $\begin{array}{c}\text { El análisis heurístico detectó que } \\
\text { el objetivo es protegido por algún } \\
\text { tipo de WAF/IPS. }\end{array}$ \\
\hline Contenido URL & $\begin{array}{l}\text { El parámetro 'p' del método GET } \\
\text { no parece ser dinámico. } \\
\text { Las pruebas heurísticas básicas realizadas } \\
\text { indican que el parámetro 'p' no } \\
\text { son inyectables. }\end{array}$ \\
\hline Inyección SQL & No es vulnerable. \\
\hline $\begin{array}{l}\text { Test 'AND boolean- } \\
\text { based blind - WHERE } \\
\text { or HAVING clause' }\end{array}$ & $\begin{array}{c}\text { Valores reflectivos fueron encontrados y } \\
\text { filtrados. Reflective value(s) found and } \\
\text { filtering out. } \\
\text { Significa que existen valores "reflexivos" } \\
\text { dentro de la respuesta que contiene } \\
\text { (partes de) la carga útil. Esto es notablemente } \\
\text { malo en algunos casos, especialmente en } \\
\text { inyecciones booleanas. }\end{array}$ \\
\hline $\begin{array}{l}\text { Test UNION con consulta } \\
\text { NULL y Método heurístico } \\
\text { con parámetro 'User-Agent' }\end{array}$ & $\begin{array}{l}\text { El parámetro 'p' del método GET no parece } \\
\text { ser dinámico. } \\
\text { Las pruebas heurísticas básicas realizadas } \\
\text { indican que el parámetro 'p' no son inyectables } \\
\text { Parámetro User-Agent no es inyectable. } \\
\text { Parámetro 'Referer' no parece ser dinámico. } \\
\text { En el análisis heurístico el parámetro 'Referer' } \\
\text { parece no ser inyectable. } \\
\text { El parámetro HOST no parece ser dinámico. } \\
\text { En el análisis heurístico el parámetro 'Host' } \\
\text { parece no ser inyectable. }\end{array}$ \\
\hline
\end{tabular}

\subsection{Tercera prueba}

Se captura la cookie ORA_WWV-W7Hhdq_v8DH8Oli 2Fp4IsyM y se procede a utilizarla mientras la aplicación está activa. Se ejecuta el comando python3 sqlmap-dev/sqlmap.py -u "http://172.16.1.87:8080/ords/f?p=502:1:13479648228 07:::::" -tables - cookie $=$ ORA_WWV-W7Hhdq_v8DH 8Oli2Fp4IsyMR -random-agent -ignore-proxy -level 5 , incluyendo un agente aleatorio e ignorando los proxys, pues este último se lo utiliza únicamente con el propósito de capturar cookies, así como también aumentando el nivel de análisis al máximo. Se agrega el módulo de identificación de tablas. El análisis inicia el 2020-03-16 a las 12:21:44. La Tabla 4 refleja los resultados obtenidos.

\section{Discusión}

Los ataques comunes a sistemas de computación se dan por virus, gusanos y adversarios humanos [3]. La detección de un ataque por inyección SQL puede darse cuando se acostumbra a revisar verificaciones de logs, registros de acceso, detecciones de intrusos entre otras [7], [15], a las que se agrega la aplicación del principio de defensa en profundidad aplicando herramientas como IDS o WAFs [3]. Las evaluaciones continuas a las aplicaciones que se desarrollan y sus certificaciones, previo el paso a entornos en producción, se convierten en otra práctica fundamental, aspecto que usualmente se omite en las organizaciones por el intento de publicar lo antes posible.

Tabla 4. Resultados de la tercera prueba

\begin{tabular}{cc}
\hline Test & Descripción \\
\hline Conexión con el URL & $\begin{array}{c}\text { La conexión pide una redirección } \\
\text { a una nueva URL generada de } \\
\text { manera aleatoria. No se acepta } \\
\text { la misma ya que se está utilizando } \\
\text { una conexión ya establecida. }\end{array}$ \\
\hline Análisis heurístico & Se evade el WAF/IPS. \\
\hline Contenido URL & $\begin{array}{c}\text { El parámetro 'p' del método GET } \\
\text { no parece ser dinámico. } \\
\text { Las pruebas heurísticas básicas } \\
\text { Inyección SQL } \\
\text { realizadas indican que el parámetro } \\
\text { 'p' no son inyectables. }\end{array}$ \\
\hline $\begin{array}{c}\text { Test 'MySQL Boolean- } \\
\text { based blind - Parameter } \\
\text { replace (MAKE_SET)' }\end{array}$ & $\begin{array}{c}\text { Nalores reflectivos fueron encontrados } \\
\text { y filtrados. Reflective value(s) found } \\
\text { dentro de la respuesta que contiene } \\
\text { (partes de) la carga útil. Esto es }\end{array}$ \\
& notablemente malo en algunos casos, \\
& especialmente en inyecciones booleanas. \\
\hline
\end{tabular}

La inyección SQL no es una técnica que se aplica con el pulsar de un botón. Se requieren conocimientos sobre el lenguaje SQL. Agrega Clarke [8] que el uso de herramientas para realizar este tipo de acometidas es importante, pues permiten automatizar el ataque. La combinación de herramientas permite obtener resultados más precisos. Concordando con Santin et al. [7] y Ojagbule et al. [5], la aplicación de la herramienta SQLMAP para el análisis fue seleccionada por la popularidad, la disponibilidad y el acceso a sus diversas distribuciones, comprobando que herramientas basadas en FOSS permiten lograr resultados tan interesantes como el usar herramientas de pago.

Se ha evidenciado que en la base de datos de exploits (https://www.exploit-db.com), el último mecanismo para vulnerar un sistema hecho en APEX fue liberado el 16 de abril del 2009.

A diferencia del trabajo realizado por Clarke (2009) en el que se utiliza una aplicación vulnerable a propósito (en inglés damn vulnerableweb site), desarrollada en PHP con motor de datos MySQL cuyo objetivo es proporcionar una plataforma de pruebas a profesionales que requieran probar sus niveles de destrezas y conocimientos.

El resultado de la prueba 'AND boolean-based blind - WHERE or HAVING clause' se puede ejemplificar en una página estática, excepto con una pequeña parte donde refleja el valor del parámetro probado (el mismo donde el SQLMap realiza la inyección). En 
caso de que dicho contenido "reflexivo" no se detecte y neutralice, existe una posibilidad considerable de que la respuesta aparezca como un cambio debido a las cargas útiles de inyección SQL utilizadas (por ejemplo, AND $2>3$ ). Por lo tanto, surge el riesgo de detección de falsos positivos (o falsos negativos en algunos casos).

La herramienta, en la primera ejecución, culmina con el argumento de que todos los parámetros evaluados no parecen inyectables, sugiere aumentar el nivel y el riesgo si se desea realizar más pruebas. En sospecha de algún tipo de mecanismo de protección involucrado (ej. WAF), se podría usar la opción '-tamper' (e.g. '-tamper $=$ space2comment') y/o cambiarlo por '-random-agent'. El ajuste en la prueba 3 con la configuración del parámetro NIVEL $=5$ fue necesario para que SQLMAP realice la prueba de vulnerabilidad de cookies.

La detección y prevención, según [21] se convierte en una tarea difícil si no se comprende adecuadamente el concepto de este tipo de ataques. Realizar evaluaciones binarias, tal como lo proponen [29] podría considerarse como una alternativa de mitigación, ya que se trata de un método extremadamente automatizado que detecta y bloquea ataques de inyección SQL en aplicaciones web.

Frente a ataques de inyección SQL a ciegas, la técnica más popular es AMNESIA (de las siglas en inglés Analysis and Monitoring for Neutralizing SQLinjection Attacks) [30], que es una herramienta aplicable únicamente para proteger las aplicaciones basadas en JAVA y que utilizan monitoreo en tiempo de ejecución [21]. Esta herramienta utiliza algoritmos de aprendizaje de máquina para proveer mecanismos de prevención y detección de amenazas Blind SQL Injection.

Otro mecanismo de prevención es el algoritmo de identificación de patrones, propuesto por Aho-Corasick [31], algoritmo que tiene dos fases: i) una fase estática y; ii) una fase dinámica.

En la fase estática, según [32], las consultas SQL generadas por el usuario son comparados con una lista de patrones que contienen una muestra de los patrones de ataque más conocidos. Si la sentencia SQL concuerda exactamente con uno de los patrones dados en la lista de patrones estáticos, significa que se está intentando un ataque SQL.

Otra alternativa que propone [31] citando a [33] es la SQLRand, donde la idea básica es generar sentencias SQL con el uso de comandos aleatorios en el que la plantilla de consulta dentro de la aplicación pueda ser aleatorizada. En esta forma, los comandos SQL que son inyectados por usuarios maliciosos no son codificados, por cuanto el proxy no reconoce los comandos inyectados haciendo que el ataque no se lleve a cabo.

Adicionalmente, [31] mencionando a [34], indica que existe un método adicional conocido como el Método de consulta por Token (Query Tokenization Method), en el que se genera un token de la consulta original y de la consulta con inyección. Luego, los tokens resultantes de este proceso se almacenan en un arreglo. La longitud de arreglos obtenidos de la consulta original y de la consulta con inyección son comparadas, si existe una coincidencia entonces no existe un intento de inyección SQL, caso contrario, se trata de un ataque.

A la lista de opciones de mitigación se suma la propuesta de [35] que consiste en un enfoque de validación de árbol gramatical, representado en una sentencia. Analizar gramaticalmente una sentencia requiere del conocimiento de la gramática del lenguaje en el que está escrita la sentencia. De tal manera que, cuando el atacante inyecte una consulta SQL maliciosa, entonces el árbol gramatical de la consulta original y la consulta con inyección no coinciden. En esta técnica, la sentencia en particular y la sentencia original son comparadas en tiempo de ejecución.

No está demás indicar que la codificación segura es crucial para el diseño de software y sistemas computacionales, aspecto que es omitido por los desarrolladores [12] debido en gran parte al desconocimiento de estándares de codificación segura, negligencia y pérdida de desempeño, a las que se suman las situaciones de usabilidad [36].

\section{Conclusiones}

Una de las características más notables de Oracle APEX es la de crear sesiones con cookies y enlaces URL al software con datos aleatorios. Las pruebas realizadas con la ejecución de las diferentes opciones de los comandos indicadas en esta técnica no permitieron acometer el software con la técnica de inyección SQL a una solución desarrollada en esta plataforma. Si bien una cookie puede ser capturada con Burp Suite, descifrar la misma toma un tiempo considerable. Sin embargo, en ese lapso, Oracle APEX dinámicamente ya generó una nueva cookie haciendo que el ataque por esta técnica sea básicamente imposible.

La contribución de este artículo ha sido el de evaluar diferentes técnicas de inyección SQL para enfatizar sobrescritura de código seguro, optimizar las etiquetas por omisión que se generan y así mejorar el nivel de seguridad de una aplicación desarrollada en Oracle APEX, sin olvidar que las pruebas han sido desarrolladas en un lapso temporal, sin eximir vulnerabilidades latentes que puedan presentarse en el día 0 .

\section{Referencias}

[1] A. Barinas López, A. C. Alarcón Aldana, and M. Callejas Cuervo, "Vulnerabilidad de ambientes virtuales de aprendizaje utilizando SQLMAP, RIPS, W3AF y Nessus," Ventana Informática, no. 30, 
pp. 247-260, 2014. [Online]. Available: https: //doi.org/10.30554/ventanainform.30.276.2014

[2] S. Mohammadi and A. Namadchian, "Anomalybased Web Attack Detection: The Application of Deep Neural Network Seq2Seq With Attention Mechanism," The ISC International Journal of Information Security, vol. 12, no. 1, pp. 44-54, 2020. [Online]. Available: http: //doi.org/10.22042/ISECURE.2020.199009.479

[3] K. L. Ingham, A. Somayaji, J. Burge, and S. Forrest, "Learning DFA representations of HTTP for protecting web applications," Computer Networks, vol. 51, no. 5, pp. 1239-1255, 2007, from Intrusion Detection to Self-Protection. [Online]. Available: https://doi.org/10.1016/j.comnet.2006.09.016

[4] B. Dwan, "The Computer Virus - From There to Here.: An Historical Perspective." Computer Fraud \& Security, vol. 2000, no. 12 , pp. 13-16, 2000. [Online]. Available: https://doi.org/10.1016/S1361-3723(00)12026-3

[5] O. Ojagbule, H. Wimmer, and R. J. Haddad, "Vulnerability Analysis of Content Management Systems to SQL Injection Using SQLMAP," in SoutheastCon 2018, 2018, pp. 1-7. [Online]. Available: https://doi.org/10.1109/SECON.2018.8479130

[6] C. Kruegel, G. Vigna, and W. Robertson, "A multi-model approach to the detection of web-based attacks," Computer Networks, vol. 48, no. 5, pp. 717-738, 2005, web Security. [Online]. Available: https://doi.org/10.1016/j.comnet.2005.01.009

[7] F. Santin, J. A. Oliveira de Figueiredo, and V. Lago Machado, "Uso da ferramenta sqlMap para detecção de vulnerabilidades de SQL Injection," in Anais do EATI - Encontro Anual de Tecnologia da Informação, 2017. [Online]. Available: https://bit.ly/340cKP6

[8] J. Clarke, SQL Injection Attacks and Defense (Second Edition), second edition ed., J. Clarke, Ed. Boston: Syngress, 2012. [Online]. Available: https: //doi.org/10.1016/B978-1-59-749963-7.00012-8

[9] D. E. Nofal and A. Amer, SQL Injection Attacks Detection and Prevention Based on Neuro-Fuzzy Technique. Springer, Cham, 2019. [Online]. Available: https: //doi.org/10.1007/978-3-030-31129-2_66

[10] B. Bin Halib, E. Budiman, and H. Jati Setyadi, "Teknik Hacking Web Server Dengan SQLMAP Di Kali Linux," Jurnal Rekayasa Teknologi Informasi, vol. 1, no. 1, pp. 67-72, 2017. [Online]. Available: http://dx.doi.org/10.30872/jurti.v1i1.642
[11] OWASP. (2017) lobally recognized by developers as the first step towards more secure coding. [Online]. Available: https://bit.ly/2JTb9DF

[12] S. Kals, E. Kirda, C. Kruegel, and N. Jovanovic, "SecuBat: A Web Vulnerability Scanner," in Proceedings of the 15th International Conference on World Wide Web, ser. WWW '06. New York, NY, USA: Association for Computing Machinery, 2006, pp. 247-256. [Online]. Available: https://doi.org/10.1145/1135777.1135817

[13] J. Fonseca, M. Vieira, and H. Madeira, "Testing and Comparing Web Vulnerability Scanning Tools for SQL Injection and XSS Attacks," in 13th Pacific Rim International Symposium on Dependable Computing (PRDC 2007), 2007, pp. 365-372. [Online]. Available: https://doi.org/10.1109/PRDC.2007.55

[14] E. B. Setiawan and A. Setiyadi, "Web vulnerability analysis and implementation," IOP Conference Series: Materials Science and Engineering, vol. 407, p. 012081, sep 2018. [Online]. Available: https://doi.org/10.1088\% 2F1757-899x\%2F407\%2F1\%2F012081

[15] J. Atoum and A. Qaralleh, "A hybrid technique for SQL injection attacks detection and prevention," International Journal of Database Management Systems ( IJDMS, vol. 6, no. 1, pp. 21-28, 2014. [Online]. Available: http://doi.org/10.5121/ijdms.2014.6102

[16] D. Herrmann and H. Pridöhl, Basic Concepts and Models of Cybersecurity, 2020, vol. 21. [Online]. Available: https://doi.org/ 10.1007/978-3-030-29053-5_2

[17] AVI Network. (2020) SQL Injection Attack. [Online]. Available: https://bit.ly/3mb96YF

[18] P. Ramasamy and S. Abburu, "SQL Injection Attack: Detection and Prevention," International Journal of Engineering Science and Technology, vol. 4, no. 4, pp. 1396-1401, 2016. [Online]. Available: https://bit.ly/3n7aSeV

[19] XS Code. (2020) XS:Code. [Online]. Available: https://bit.ly/37MYc6s

[20] D. Novski Neto, "Web (eternamente) revisitada : análise de vulnerabilidades web e de ferramentas de código aberto para exploração," 2019. [Online]. Available: https://bit.ly/37VrNui

[21] V. K. Gudipati, T. Venna, S. Subburaj, and O. Abuzaghleh, "Advanced automated SQL injection attacks and defensive mechanisms," in 2016 Annual Connecticut Conference on 
Industrial Electronics, Technology Automation (CT-IETA), 2016, pp. 1-6. [Online]. Available: https://doi.org/10.1109/CT-IETA.2016.7868248

[22] C. Cetin, D. Goldgof, and J. Ligatti, "SQLIdentifier Injection Attacks," in 2019 IEEE Conference on Communications and Network Security (CNS), 2019, pp. 151-159. [Online]. Available: https://doi.org/10.1109/CNS.2019.8802743

[23] J. P. Singh, "Analysis of SQL Injection Detection Techniques," 2016. [Online]. Available: https://bit.ly/375XeDh

[24] O. Ojagbule, H. Wimmer, and R. J. Haddad, "Vulnerability Analysis of Content Management Systems to SQL Injection Using SQLMAP," in SoutheastCon 2018, 2018, pp. 1-7. [Online]. Available: https://doi.org/10.1109/SECON.2018.8479130

[25] A. Ciampa, C. A. Visaggio, and M. Di Penta, "A Heuristic-Based Approach for Detecting SQL-Injection Vulnerabilities in Web Applications," in Proceedings of the 2010 ICSE Workshop on Software Engineering for Secure Systems, ser. SESS '10. New York, NY, USA: Association for Computing Machinery, 2010, pp. 43-49. [Online]. Available: https://doi.org/10.1145/1809100.1809107

[26] R. Alsahafi, "SQL Injection Detection and Prevention Techniques," International Journal of Scientific \& Technology Research, vol. 8, no. 1, pp. 182-185, 2019. [Online]. Available: https://bit.ly/2W24Ksp

[27] L. Wichman, "Mass SQL injection for malware distribution," SANS Institute, Tech. Rep., 2011. [Online]. Available: https://bit.ly/2Ke3ks0

[28] JAVANICUS. (2016) Posts Related to Web-Pentest-SQL-Injection. [Online]. Available: https://bit.ly/2IEFUMc

[29] V. Sunkari and C. V. Guru rao, "Protect Web Applications against SQL Injection Attacks Using Binary Evaluation Approach," International Journal of Innovations in Engineering and Technology (IJIET), pp. 484-490, 2016. [Online]. Available: https://bit.ly/377eVSR

[30] W. G. J. Halfond and A. Orso, "AMNESIA: Analysis and Monitoring for NEutralizing
SQL-Injection Attacks," in Proceedings of the 20th IEEE/ACM International Conference on Automated Software Engineering, ser. ASE '05. New York, NY, USA: Association for Computing Machinery, 2005, pp. 174-183. [Online]. Available: https://doi.org/10.1145/1101908.1101935

[31] M. A. Prabakar, M. KarthiKeyan, and K. Marimuthu, "An efficient technique for preventing SQL injection attack using pattern matching algorithm," in 2013 IEEE International Conference ON Emerging Trends in Computing, Communication and Nanotechnology (ICECCN), 2013, pp. 503-506. [Online]. Available: https: //doi.org/10.1109/ICE-CCN.2013.6528551

[32] G. Yiğit and M. Arnavutoğlu, "SQL Injection Attacks Detection \& Prevention Techniques," International Journal of Computer Theory and Engineering, vol. 9, no. 5, pp. 351-356, 2017. [Online]. Available: https://bit.ly/3qKrEm5

[33] S. W. Boyd and A. D. Keromytis, "Boyd s.w., keromytis a.d." in International Conference on Applied Cryptography and Network Security, 2004, pp. 292-302. [Online]. Available: https://doi.org/10.1007/978-3-540-24852-1_21

[34] L. Ntagwabira and S. L. Kang, "Use of Query tokenization to detect and prevent SQL injection attacks," in 2010 3rd International Conference on Computer Science and Information Technology, vol. 2, 2010, pp. 438-440. [Online]. Available: https://doi.org/10.1109/ICCSIT.2010.5565202

[35] G. Buehrer, B. W. Weide, and P. A. G. Sivilotti, "Using Parse Tree Validation to Prevent SQL Injection Attacks," in Proceedings of the 5th International Workshop on Software Engineering and Middleware, ser. SEM '05. New York, NY, USA: Association for Computing Machinery, 2005, pp. 106-113. [Online]. Available: https://doi.org/10.1145/1108473.1108496

[36] F. D. Nembhard, M. M. Carvalho, and T. C. Eskridge, "Towards the application of recommender systems to secure coding," EURASIP Journal on Information Security, vol. 2019, no. 1, p. 9, Jun. 2019. [Online]. Available: https://doi.org/10.1186/s13635-019-0092-4 


\section{Normas PARA PUBLICAR EN LA REVISTA INGENIUS}

\section{Información General}

INGENIUS es una publicación científica de la Universidad Politécnica Salesiana de Ecuador, editada desde enero de 2007, con periodicidad fija semestral, especializada en Ingeniería Mecánica, Ingeniería Eléctrica, Electrónica, Ciencias de la Computación y su integración en lo que actualmente se conoce como Mecatrónica; estas líneas de acción fortalecen áreas como automatización, control, robótica entre otras.

Es una revista científica arbitrada, que utiliza el sistema de evaluación externa por expertos (peer-review), bajo metodología de pares ciegos (doble-blind review), conforme a las normas de publicación del Institute of Electrical and Electronics Engineers (IEEE). El cumplimiento de este sistema permite garantizar a los autores un proceso de revisión objetivo, imparcial y transparente, lo que facilita a la publicación su inclusión en bases de datos, repositorios e indexaciones internacionales de referencia.

INGENIUS se encuentra indexada en el directorio y catálogo selectivo del Sistema Regional de Información en Línea para Revistas Científicas de América Latina, el Caribe, España y Portugal - Latindex, en el Directorio de Revistas de Acceso Abierto - DOAJ, en la Matriz de Información para el Análisis de Revistas MIAR, en la Red Iberoamericana de Innovación y Conocimiento Científico - REDIB y en repositorios, bibliotecas y catálogos especializados de Iberoamérica.

La revista se edita en doble versión: impresa (ISSN: 1390-650X) y electrónica (e-ISSN: 1390-860X), en idioma español, siendo identificado además cada trabajo con un DOI (Digital
Object Identifier System). Los artículos enviados a la revista InGEnIUs deben ajustarse a los siguientes criterios:

\section{Alcance y Política}

\subsection{Temática}

Contribuciones originales en materia de Ingeniería Mecánica, Ingeniería Eléctrica y Electrónica, Ciencias de la computación y su integración en lo que actualmente se conoce como Mecatrónica, así como áreas afines: Automatización, Control, Domótica, Robótica en sus diferentes ámbitos de acción y todas aquellas disciplinas conexas interdisciplinarmente con la línea temática central.

Podrán ser publicados todos los trabajos realizados por investigadores nacionales o extranjeros, una vez que cumplan los criterios de calidad científica requeridos.

\subsection{Aportaciones}

La revista INGENIUS publica preferentemente artículos relacionados con investigaciones empíricas, siendo también admisibles informes de desarrollo tecnológico, propuestas de modelos e innovaciones, productos de la elaboración de tesis de grado y posgrado siempre que sean un aporte para el campo de la ciencia y tecnología, así como selectas revisiones del estado del arte (state-of-the-art).

- Investigaciones: 5.000 a 6.500 palabras de texto, incluyendo título, resúmenes, palabras clave, tablas y referencias.

- Informes, propuestas y productos: 5.000 a 6.500 palabras de texto, incluyendo título, resúmenes, tablas y referencias.

- Revisiones: 6.000 a 7.000 palabras de texto, incluidas tablas y referencias. Se 
valora especialmente las referencias justificadas, actuales y selectivas de alrededor de unas 40 obras.

La revista INGENIUS publica trabajos originales e inéditos redactados en español e inglés, no pueden haber sido publicados a través de ningún medio impreso ni electrónico, ni estar en proceso de arbitraje o publicación.

Todo artículo será sometido a un riguroso proceso de arbitraje; la evaluación del artículo se hará conforme a criterios de originalidad, pertinencia, actualidad, aportes, rigurosidad científica y cumplimiento de las normas editoriales establecidas.

Por tratarse de una publicación arbitrada, el Consejo Editorial aprueba su publicación en base al concepto de pares especializados. La recepción de un documento no implica compromiso de publicación.

Es indispensable presentar una carta de presentación y cover letter que se puede descargar de: <https://goo.gl/xB0wEl>.

Las contribuciones deben ser enviadas única y exclusivamente a través del OJS (Open Journal System) <https://goo.gl/4xxjuo $>$, en el cual todos los autores deben registrarse como usuario previamente. Para cualquier consulta del procedimiento se debe contactar a:

$<$ revistaingenius@ups.edu.ec $>$,

$<$ jcalle@ups.edu.ec $>$ ó

$<$ mquinde@ups.edu.ec $>$.

\section{Presentación y estructura de los manuscritos}

Para aquellos trabajos que se traten de investigaciones de carácter empírico, los manuscritos seguirán la estructura IMRDC (Introducción, Materiales y Métodos, Resultados y Discusión y Conclusiones), siendo opcionales los epígrafes de Notas y Apoyos. Aquellos trabajos que por el contrario se traten de informes, estudios, propuestas y revisiones podrán ser más flexibles en sus epígrafes, especialmente en Materiales y métodos, Resultados, y Discusión y Conclusiones. En todas las tipologías de trabajos son obligatorias las Referencias.

Los artículos pueden estar escritos sobre Microsoft Word (.doc o .docx) o $\mathrm{AT}_{\mathrm{EX}}$ (.tex). La plantilla a ser utilizada puede ser descargada del sitio web de la revista, en formato de Microsoft Word en: <https://goo.gl/ZA2XAk>, mientras que para $\mathrm{AT}_{\mathrm{E} X}$ en: $<$ https://goo gl/Mwv8IC > , es necesario que el archivo esté anonimizado en Propiedades de Archivo, de forma que no aparezca la identificación de autor/es.

Las Figuras, Gráficos y/o Ilustraciones, así como las Tablas deberán estar numeradas secuencialmente incluyendo una descripción explicativa para cada una. Las ecuaciones incluidas en el artículo deberán también estar numeradas; tanto las figuras, tablas y ecuaciones deben estar citadas en el texto.

Use espacio después de punto, comas y signos de interrogación.

Use "enter" al final de cada párrafo, título encabezamiento. No use "enter" en ningún otro lugar, deje al programa procesador de palabras romper automáticamente las líneas.

No centre encabezamientos o subencabezamientos ya que deben estar alineados a la izquierda.

Las Tablas deben estar creadas en el mismo programa usado para el cuerpo del documento. Use tabuladores, no espacios, para crear columnas. Recuerde que el tamaño final de las páginas impresas será de 21 x $28 \mathrm{~cm}$, por lo tanto las tablas deben estar diseñadas para ajustarse al espacio de la impresión final.

\subsection{Estructura de los manuscritos}

\subsubsection{Presentación y Cover Letter}

1. Título (español) / Title (inglés): Conciso pero informativo, en castellano en primera línea y en inglés en segunda, cuando el artículo sea escrito en español 
y viceversa si está escrito en inglés.

2. Autores y adscripción: Nombre y Apellidos completo de cada autor, organizados por orden de prelación y su adscripción institucional con referencia al final de la primera hoja, donde tiene que incluir: Dependencia a la que pertenece, Institución a la que pertenece, país, ORCID. Se aceptarán como máximo 5 autores, aunque pudieran existir excepciones justificadas por la complejidad y extensión del tema.

\section{Resumen (español) / Abstract} (inglés): Tendrá como extensión máxima 230 palabras, en español y en inglés. En el resumen se describirá de forma concisa y en este orden: 1) Justificación del tema; 2) Objetivos; 3) Metodología y muestra; 4) Principales resultados; 5) Principales conclusiones.

\section{Palabras clave (español) / Key-} words (inglés): Se deben exponer 6 palabras clave por cada versión idiomática relacionados directamente con el tema del trabajo. Será valorado positivamente el uso de las palabras claves expuestas en el Thesaurus de la UNESCO.

5. Presentación (Cover Letter): Una declaración de que el manuscrito se trata de una aportación original, no enviada ni en proceso de evaluación en otra revista, con la confirmación de las autorías firmantes, aceptación (si procede) de cambios formales en el manuscrito conforme a las normas y cesión parcial de derechos a la editorial, según el formato establecido en: $<$ https://goo.gl/XAc9a3 $>$.

\subsubsection{Manuscrito}

1. Título (español) / Title (inglés): Conciso pero informativo, en castellano en primera línea y en inglés en segunda, cuando el artículo sea escrito en español y viceversa si está escrito en inglés.

2. Autores y adscripción: Nombre y Apellidos completo de cada autor, organizados por orden de prelación y su adscripción institucional con referencia al final de la primera hoja, donde tiene que incluir: Dependencia a la que pertenece, Institución a la que pertenece, país, ORCID. Se aceptarán como máximo 5 autores, aunque pudieran existir excepciones justificadas por la complejidad y extensión del tema.

3. Resumen (español) / Abstract (inglés): Tendrá como extensión máxima 230 palabras, en español y en inglés. En el resumen se describirá de forma concisa y en este orden: 1) Justificación del tema; 2) Objetivos; 3) Metodología y muestra; 4) Principales resultados; 5) Principales conclusiones.

4. Palabras clave (español) / Keywords (inglés): Se deben exponer 6 palabras clave por cada versión idiomática relacionados directamente con el tema del trabajo. Será valorado positivamente el uso de las palabras claves expuestas en el Thesaurus de la UNESCO.

5. Introducción: Debe incluir el planteamiento del problema, el contexto de la problemática, la justificación, fundamentos y propósito del estudio, utilizando citas bibliográficas, así como la literatura más significativa y actual del tema a escala nacional e internacional.

6. Materiales y métodos: Debe ser redactado de forma que el lector pueda comprender con facilidad el desarrollo de la investigación. En su caso, describirá la metodología, la muestra y la forma de muestreo, así como se hará referencia al tipo de análisis estadístico empleado. Si se trata de una metodología original, es 
necesario exponer las razones que han conducido a su empleo y describir sus posibles limitaciones.

7. Análisis y resultados: Se procurará resaltar las observaciones más importantes, describiéndose, sin hacer juicios de valor, el material y métodos empleados. Deberán aparecer en una secuencia lógica en el texto y las tablas y figuras imprescindibles evitando la duplicidad de datos.

8. Discusión y Conclusiones: Resumirá los hallazgos más importantes, relacionando las propias observaciones con estudios de interés, señalando aportaciones y limitaciones, sin redundar datos ya comentados en otros apartados. Asimismo, debe incluir las deducciones y líneas para futuras investigaciones.

9. Apoyos y agradecimientos (opcionales): El Council Science Editors recomienda al autor/es especificar la fuente de financiación de la investigación. Se considerarán prioritarios los trabajos con aval de proyectos competitivos nacionales e internacionales.

10. Las notas (opcionales): Se deberán incluir solo en caso necesario, al final del artículo (antes de las referencias). Deben anotarse manualmente, ya que el sistema de notas al pie o al final de Word no es reconocido por los sistemas de maquetación. Los números de notas se colocan en superíndice, tanto en el texto como en la nota final. No se permiten notas que recojan citas bibliográficas simples ( $\sin$ comentarios), pues éstas deben ir en las referencias.

11. Referencias Bibliográficas: Las citas bibliográficas deben reseñarse en forma de referencias al texto. Bajo ningún caso deben incluirse referencias no citadas en el texto. Su número debe ser suficiente para contextualizar el marco teórico con criterios de actualidad e importancia. Se presentarán secuencialmente en orden de aparición, según corresponda siguiendo el formato de la IEEE.

\subsection{Normas para las referencias Bibliográficas}

Artículos de revistas:

[1] J. Riess, J. J. Abbas, "Adaptive control of cyclic movements as muscles fatigue using functional neuromuscular stimulation". IEEE Trans. Neural Syst. Rehabil. Eng vol. 9, pp.326-330, 2001. [Onine]. Available: https://doi.org/10.1109/7333.948462 Libros:

[1] G. O. Young, "Synthetic structure of industrial plastics" in Plastics, 2nd ed., vol. 3, J. Peters, Ed. New York: McGraw-Hill, 1964, pp. 15-64.

Reportes Técnicos:

[1] M. A. Brusberg and E. N. Clark, "Installation, operation, and data evaluation of an oblique-incidence ionosphere sounder system," in "Radio Propagation Characteristics of the Washington-Honolulu Path," Stanford Res. Inst., Stanford, CA, Contract NOBSR-87615, Final Rep., Feb. 1995, vol. 1

Artículos presentados en conferencias (No publicados):

[1] Vázquez, Rolando, Presentación curso "Realidad Virtual". National Instruments. Colombia, 2009.

Artículos de Memorias de Conferencias (Publicados):

[1] L. I. Ruiz, A. García, J. García, G. Taboada. "Criterios para la optimización de sistemas eléctricos en refinerías de la industria petrolera: influencia y análisis en el equipo eléctrico," IEEE CONCAPAN XXVIII, Guatemala 2008. 
Tesis:

[1] L.M. Moreno, "Computación paralela y entornos heterogéneos," Tesis doctoral, Dep. Estadística, Investigación Operativa y Computación, Universidad de La Laguna, La Laguna, 2005.

\section{Estándares:}

[1] IEEE Guide for Application of Power Apparatus Bushings, IEEE Standard C57.19.100-1995, Aug. 1995.

Patentes:

[1] J. P. Wilkinson, "Nonlinear resonant circuit devices," U.S. Patent 3624 125, July $16,1990$.

Manuales:

[1] Motorola Semiconductor Data Manual, Motorola Semiconductor Products Inc., Phoenix, AZ, 1989.

Recursos de internet:

[1] E. H. Miller, "A note on reflector arrays" [Online]. Available: https://goo.gl/4cJkCF

\subsection{Epígrafes, tablas y figuras}

Los epígrafes del cuerpo del artículo se deben numerar en arábigo. Irán sin caja completa de mayúsculas, ni subrayados, ni negritas. La numeración ha de ser como máximo de tres niveles: 1. / 1.1. / 1.1.1. Al final de cada epígrafe numerado se dará un enter para continuar con el párrafo correspondiente.

Las tablas deben presentarse incluidas en el texto según orden de aparición, numeradas en arábigo y subtituladas con la descripción del contenido, el subtítulo debe ir en la parte superior de la tabla justificado a la izquierda.

Las figuras pueden ser dibujos lineales, mapas o fotografías de medios tonos en blanco y negro o a color en resolución de 300 dpi. No combine fotografías y dibujos lineales en la misma figura.

Diseñe las figuras para que se ajusten eventualmente al tamaño final de la revista 21 x 28 $\mathrm{cm}$. Asegúrese de que las inscripciones o detalles, así como las líneas, tengan tamaños y grosores adecuados de tal manera que no queden ilegibles cuando sean reducidos a su tamaño final (números, letras y símbolos deben ser reducidos al menos a 2,5 mm de altura después que las ilustraciones han sido reducidas para ajustarse a la página impresa). Idealmente, las ilustraciones lineales deben ser preparadas a aproximadamente un cuarto de su tamaño final de publicación. Diferentes elementos en la misma figura deben ser deletreados a, b, c, etc.

Las fotografías deben grabarse con alto contraste y en alta resolución. Recuerde que las fotografías frecuentemente pierden contraste en el proceso de impresión.

Dibujos lineales y mapas deben ser preparados en color negro.

El texto de las figuras y mapas debe escribirse con letras fácilmente legibles.

Si las figuras han sido previamente usadas, es de responsabilidad del autor obtener el permiso correspondiente para evitar problemas posteriores relacionados con los derechos de autor.

Cada figura debe ser entregada en un archivo aparte, ya sea como mapa de bits (.jpg, .bmp, .gif, o .png) o como gráfico vectorial (.ps, .eps, .pdf).

\section{Proceso de envío}

El envío será a través de la plataforma OJS de la revista, <https://goo.gl/4xxjuo>, de manera obligatoria dos archivos se deberán cargar, como archivo original el manuscrito en .pdf sin datos de autor y anonimizado, mientras que en archivos complementarios se deberá cargar la presentación y cover letter de acuerdo a lo antes descrito, adicionalmente se deberá cargar el manuscrito completo en .doc o .docx (archivo de Word), es decir con los datos del autor/es y su adscripción institucional; también se deben cargar en archivos independientes las 
figuras numeradas de acuerdo a lo correspondiente en el manuscrito (como mapa de bits .jpg, .bmp, .gif, o .png o como gráfico vectorial .ps, .eps, .pdf), en calidad como mínima de 300 dpi.

Todos los autores deben ingresar la información requerida en la plataforma OJS y uno solo de los autores será el responsable de correspondencia.

Una vez enviada la contribución el sistema automáticamente enviará al autor para correspondencia un mail de confirmación de recepción de la contribución.

\section{Proceso Editorial}

Una vez que el manuscrito ha sido receptado en OJS se realiza una primera comprobación de los siguientes puntos:

- La temática se encuentre en concordancia con los criterios de la revista.

- Debe tener la estructura IMRDC

- Debe estar en el formato de la revista INGENIUS.

- Debe utilizar la forma de citación de la IEEE.

- Todas las referencias bibliográficas deben estar citadas en el texto del manuscrito al igual que las tablas, figuras y ecuaciones.

- El manuscrito es original, para esto se utiliza un software para determinar plagio.

La comprobación antes descrita puede llevar hasta 4 semanas.

Si alguno de los puntos anteriores no está completo o existe inconsistencia, se solicitará al autor las correcciones correspondientes, una vez que el autor modifique el artículo deberá remitir a través de la plataforma OJS. El equipo editorial verificará que las correcciones solicitadas han sido incorporadas, si cumple, el manuscrito iniciará la segunda parte del proceso y podrá ser seguido por el autor a través de OJS, caso contrario se notificará al autor y se archivará el manuscrito.
La segunda fase del proceso consiste en la evaluación bajo la metodología de pares ciegos (doble-blind review), en los que se incluyen expertos nacionales y extranjeros considerando los siguientes pasos:

- El editor asigna dos o más revisores para el artículo.

- Una vez revisado el artículo, los revisores remitirán el informe de la evaluación, con uno de los siguientes resultados.

- Publicable

- Publicable con cambios sugeridos

- Publicable con cambios obligatorios

- No Publicable

- El editor una vez recibida la evaluación por parte de los revisores analizará los resultados y determinará si el artículo es aceptado o negado.

- Si el artículo es aceptado se notificará al autor para que realice las correcciones en caso de ser requeridas.

- Si el artículo es negado se notificará al autor y se archivará el manuscrito.

- En los dos casos anteriores se enviará el resultado de la evaluación de los revisores y sus respectivas recomendaciones.

La segunda fase del proceso dura 4 semanas como mínimo, luego de transcurridas las mismas se notificará al autor dando instrucciones para continuar con el proceso.

\section{Publicación}

La revista INGENIUS publica dos números por año, el $1^{\circ}$ de enero y el $1^{\circ}$ de julio por lo tanto es importante considerar las fechas para el envío de los artículos y su correspondiente publicación. Los artículos recibidos hasta el mes de octubre serán considerados para la publicación de enero y aquellos que se reciban hasta abril para la publicación de julio. 


\title{
UNIVERSIDAD POLITÉCNICA SALESIANA DEL ECUADOR
}

Juan Cárdenas Tapia, sdb

Rector

\author{
(C)Universidad Politécnica Salesiana \\ Turuhuayco 3-69 y Calle Vieja \\ Casilla postal 2074 \\ Cuenca, Ecuador \\ Teléfono: $(+5937) 2050000$ \\ Fax: (+593 7) 4088958
}

Correo electrónico: srector@ups.edu.ec

\section{CANJE}

Se acepta canje con otras publicaciones periódicas.

Dirigirse a:

Secretaría Técnica de Comunicación

Universidad Politécnica Salesiana

Turuhuayco 3-69 y Calle Vieja

Casilla postal 2074

Cuenca, Ecuador

Teléfono: (+593 7) 2050000 Ext. 1182

Fax: (+593 7) 4088958

Correo electrónico: rpublicas@ups.edu.ec

www.ups.edu.ec

Cuenca - Ecuador

Ingenius, Revista de Ciencia y Tecnología, publicación semestral, N. ${ }^{\circ}$ 25, enero/junio 2021

John Calle Sigüencia, PhD, Editor Jefe

revistaingenius@ups.edu.ec

\section{Impresión}

Centro Gráfico Salesiano: Antonio Vega Muñoz 10-68 y General Torres.

Teléfono: (+593 7) 2831745

Cuenca - Ecuador

Correo electrónico: centrograficosalesiano@lns.com.ec 
